\title{
Total Synthesis and Absolute Stereochemical Assignment of (+)- and (-)-Galbulimima Alkaloid 13
}

\author{
Mohammad Movassaghi*, Diana K. Hunt, Meiliana Tjandra \\ Massachusetts Institute of Technology, Department of Chemistry, Massachusetts 02139
}

\section{Supporting Information}

General Procedures. All reactions were performed in oven-dried or flame-dried round bottomed flasks or modified Schlenk (Kjeldahl shape) flasks. The flasks were fitted with rubber septa and reactions were conducted under a positive pressure of argon. Stainless steel syringes or cannulae were used to transfer air- and moisture-sensitive liquids. Flash column chromatography was performed as described by Still et al. using silica gel $(60-\AA$ pore size, $32-63 \mu \mathrm{m}$, standard grade, Sorbent Technologies). ${ }^{1}$ Where necessary (so noted), silica gel was neutralized by treatment of the silica gel prior to chromatography with the eluent containing $1 \%$ triethylamine. Analytical thin-layer chromatography was performed using glass plates pre-coated with $0.25 \mathrm{~mm} \mathrm{230-400} \mathrm{mesh} \mathrm{silica} \mathrm{gel}$ impregnated with a fluorescent indicator $(254 \mathrm{~nm})$. Where necessary (so noted), silica gel plates were neutralized by treatment with a solution of $5 \%$ triethylamine in dichloromethane followed by heating on a hot plate $\left(\sim 250^{\circ} \mathrm{C}\right)$. Thin layer chromatography plates were visualized by exposure to ultraviolet light and/or by exposure to an ethanolic phosphomolybdic acid (PMA), an acidic solution of $p$-anisaldehyde (anis), an aqueous solution of ceric ammonium molybdate (CAM), an aqueous solution of potassium permanganate $\left(\mathrm{KMnO}_{4}\right)$ or an ethanolic solution of ninhydrin followed by heating $(<1 \mathrm{~min})$ on a hot plate $\left(250^{\circ} \mathrm{C}\right)$. Organic solutions were concentrated on Büchi R-200 rotary evaporators at $\sim 20$ Torr at $25-35^{\circ} \mathrm{C}$, then at $\sim 1$ Torr unless otherwise indicated.

Materials. Commercial reagents and solvents were used as received with the following exceptions: dichloromethane, diethyl ether, tetrahydrofuran, acetonitrile, and toluene were purchased from J.T. Baker $\left(\right.$ Cycletainer $\left.{ }^{\mathrm{TM}}\right)$ and were purified by the method of Grubbs et al. under positive argon pressure. $^{2}$ Triethylamine, diisopropylethylamine, and benzene were distilled over calcium hydride immediately before use. Acrolein was distilled over calcium sulfate immediately before use. Methyl vinyl ketone was distilled over potassium carbonate and calcium chloride immediately prior to use. Martin sulfurane was purchased from Aldrich and stored in a glove box under nitrogen atmosphere. $N$-Bromosuccinimide (NBS) was recrystallized from boiling water prior to use. 2-Iodoxybenzoic acid (IBX) was prepared according to literature procedure. ${ }^{3}$ Activated $\gamma$-manganese dioxide $\left(\mathrm{MnO}_{2}\right)$ was prepared according to literature procedure. ${ }^{4}$ The molarity of $n$-butyllithium solutions was determined by titration using diphenylacetic acid as an indicator (average of three determinations). ${ }^{5}$ Ammonia saturated dichloromethane was obtained by agitation of dichloromethane in the presence of ammonium hydroxide followed by drying over anhydrous sodium sulfate. Where necessary (so noted) solutions were deoxygenated by alternate freeze (liquid nitrogen)/evacuation/argon-flush/thaw cycles (FPT, three iterations) or degassed by purging with argon for several minutes.

\footnotetext{
${ }^{1}$ Still, W. C.; Kahn, M.; Mitra, A. J. Org. Chem. 1978, 43, 2923.

${ }^{2}$ Pangborn, A. B.; Giardello, M. A.; Grubbs, R. H.; Rosen, R. K.; Timmers, F. J. Organometallics 1996, $15,1518$.

${ }^{3}$ Frigerio, M.; Santagostino, M.; Sputore, S. J. Org. Chem. 1999, 64, 4537.

${ }^{4}$ Fatiadi, A. J., Synthesis 1976, 65.

${ }^{5}$ Kofron, W. G.; Baclawski, L. M. J. Org. Chem. 1976, 41, 1879.
} 
Instrumentation. Proton nuclear magnetic resonance $\left({ }^{1} \mathrm{H}\right.$ NMR) spectra were recorded with a Varian 300 Mercury or a Varian inverse probe 500 INOVA spectrometer or a Bruker inverse probe 600 Avance spectrometer. Chemical shifts are recorded in parts per million from internal tetramethylsilane on the $\delta$ scale and are referenced from the residual protium in the NMR solvent $\left(\mathrm{CHCl}_{3}: \delta 7.27, \mathrm{C}_{6} \mathrm{D}_{5} \mathrm{H}: \delta 7.16\right)$. Data is reported as follows: chemical shift [multiplicity ( $\mathrm{s}=$ singlet, $\mathrm{d}$ $=$ doublet, $\mathrm{t}=$ triplet, $\mathrm{q}=$ quartet, $\mathrm{m}=$ multiplet, $\mathrm{app}=$ apparent, $\mathrm{br}=$ broad $)$, coupling constant $(\mathrm{s})$ in Hertz, integration, assignment]. Carbon-13 nuclear magnetic resonance $\left({ }^{13} \mathrm{C} \mathrm{NMR}\right)$ spectra were recorded with a Varian 500 INOVA spectrometer or a Bruker 400 spectrometer with a Magnex Scientific superconducting magnet and are recorded in parts per million from internal tetramethylsilane on the $\delta$ scale and are referenced from the carbon resonances of the solvent $\left(\mathrm{CDCl}_{3}\right.$ : $\delta 77.2$, benzene- $d_{6}: \delta$ 128.4). Infrared data were obtained with a Perkin-Elmer 2000 FTIR and are reported as follows: [frequency of absorption $\left(\mathrm{cm}^{-1}\right)$, intensity of absorption $(\mathrm{s}=$ strong, $\mathrm{m}=$ medium, $\mathrm{w}=$ weak, $\mathrm{br}=$ broad), assignment]. Gas chromatography was performed on an Agilent Technologies 6890N Network GC System with a HP-5 5\% Phenyl Methyl Siloxane column $\left(50{ }^{\circ} \mathrm{C}, 6\right.$ min; 25 ${ }^{\circ} \mathrm{C} / \mathrm{min}$ to $250{ }^{\circ} \mathrm{C} ; 250{ }^{\circ} \mathrm{C}, 6 \mathrm{~min}$ ). We are grateful to Dr. Li Li for obtaining the mass spectroscopic data at the Department of Chemistry's Instrumentation Facility, Massachusetts Institute of Technology. High-resolution mass spectra (HRMS) were recorded on a Bruker APEX 4.7 Tesler FTMS spectrometer using electronspray ion source (ESI) or electronspray (ES).

Compound Numbering. For compounds 2, 5, 7, 16, 18, 19, 20, 21, 2-epi-20, 2-epi-16-oxo-3, the atom numbering system used is consistent with correlated atoms in the final product as numbered in the isolation papers of the natural alkaloids. ${ }^{6}$

\footnotetext{
${ }^{6}$ Ritchie, E.; Taylor, W. C. In The Alkaloids; Manske, R. H. F., Ed.; Academic Press: New York, 1967; Vol. 9, Chapter 14.
} 


\section{Additional Notes.}

\section{Synthesis of 2-epi-16-Oxohimgaline:}

Scheme S1. Synthesis of (-)-2-epi-16-oxohimgaline (2-epi-16-oxo-3). ${ }^{a}$

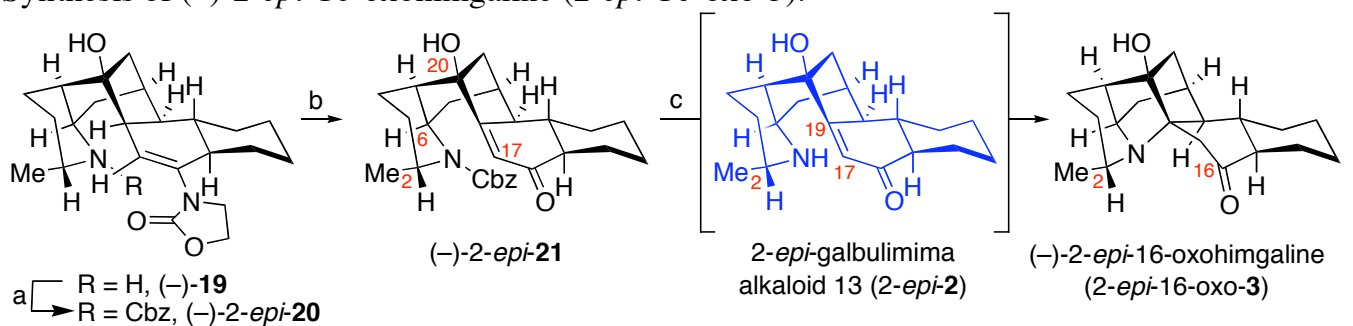

${ }^{a}$ Conditions: (a) $\mathrm{ClCO}_{2} \mathrm{Bn}, \mathrm{Na}_{2} \mathrm{CO}_{3}, \mathrm{H}_{2} \mathrm{O}, \mathrm{CH}_{2} \mathrm{Cl}_{2}, 54 \%$. (b) $\mathrm{IBX}, \mathrm{TsOH} \bullet \mathrm{H}_{2} \mathrm{O}$, benzene, DMSO, $65{ }^{\circ} \mathrm{C}, 10 \mathrm{~h}, 59 \%$. (c) TMSI, $\mathrm{CH}_{2} \mathrm{Cl}_{2}, 0{ }^{\circ} \mathrm{C} ; \mathrm{HCl} ; \mathrm{NaOH}, 23{ }^{\circ} \mathrm{C}, 93 \%$.

With access to pentacyclic amines (+)- and (-)-19 (Scheme 3), we explored the synthesis of optically active 2-epi-GB 13 (2-epi-2). Interestingly, removal of the nitrogen-protective group of (-)2-epi-N-Cbz GB 13 (2-epi-21, Scheme S1), under identical conditions to those described for $N-\mathrm{Cbz}$ GB 13 (21), led to exclusive isolation of (-)-2-epi-16-oxohimgaline (2-epi-16-oxo-3, Scheme S1, $\left.[\alpha]_{D}^{22}=-24\left(c 0.085, \mathrm{CH}_{2} \mathrm{Cl}_{2}\right)\right)$ even after treatment with base. Similarly, (+)-2-epi-16-oxohimgaline $\left([\alpha]_{D}^{22}=+24\left(c 0.07, \mathrm{CH}_{2} \mathrm{Cl}_{2}\right)\right)$ was prepared from (+)-2-epi-21. The more facile conjugate addition observed with 2-epi-GB 13 (2-epi-2), compared to that seen with GB 13 (2, Scheme 3) is likely due to decreased steric interactions between the $\mathrm{C} 2$-substituent and the $\mathrm{C} 17$-methine in the 2-epi series. This observation further supports the hypothesis for the biosynthesis of himgaline (3) via sequential conjugate addition and carbonyl reduction of GB 13 (2) as mentioned in the text.

\section{Our Proposed Biosynthesis of Class II and Class III Galbulimima Alkaloids:}

Inspired by Mander, Ritchie and Taylor's proposal on the biosynthesis of galbulimima alkaloids, ${ }^{7}$ we began our investigations in the total synthesis of Class III galbulimima alkaloids by first development of a unifying hypothesis regarding the biosynthesis of Class II and Class III galbulimima alkaloids as outlined in Scheme S2 (page S4). In particular, we envisioned a sequence of bond forming events beginning with tricycle $\mathbf{S 3}$ leading to a common pentacyclic precursor S8. We postulated that intramolecular conjugate addition of the enol tautomer of the C20-ketone of $\mathbf{S 3}$ to the unsaturated iminium ion would result in formation of tetracycle $\mathbf{S} 4$ that would be subject to rapid C6-enamine addition to the C20-carbonyl giving the pentacyclic imine S6. Imine reduction followed by enone formation to give S8 would then sets the stage for decarboxylation toward the Class III alkaloids as shown in Scheme S2. Alternatively, tautomerization of S8 and C17-hydroxylation, followed by an intramolecular allylic displacement by the amine would give the $\mathrm{N}-\mathrm{C} 9$ bond present in Class II alkaloids. Indeed, the successful implementation of our synthetic strategy for the synthesis of the CDE-ring system that was guided by our initial hypothesis regarding the biogenesis of these alkaloids provides strong incentive for us to explore the synthesis of other members of the family based on the sequence outlined in Scheme S2. During our studies directed at the synthesis of Class II and Class III alkaloids, an elegant report by Baldwin ${ }^{8}$ and co-workers employing their independent biomimetic route to himbacine and himandravine, members of the Class I alkaloids, was recently disclosed.

\footnotetext{
${ }^{7}$ Please see Scheme 1 in the following reference: Mander, L. N.; Prager, R. H.; Rasmussen, M.; Ritchie, E. Taylor, W. C. Aust. J. Chem. 1967, 20, 1705.

${ }^{8}$ (a) Tchabanenko, K.; Adlington, R. M.; Cowley, A. R.; Baldwin, J. E. Org. Lett. 2005, 7, 585. (b) For an independent and different hypothesis on the biosynthesis of Class II and Class III alkaloids, please see Scheme 11 in Tchabanenko, K.; Chesworth, R.; Parker, J. S.; Anand, N. K.; Russell, A. T.; Adlington, R. M.; Baldwin, J. E. Tetrahedron 2005, 61, 11649.
} 
Scheme S2. Our proposed biosynthesis of Class II and Class III galbulimima alkaloids.
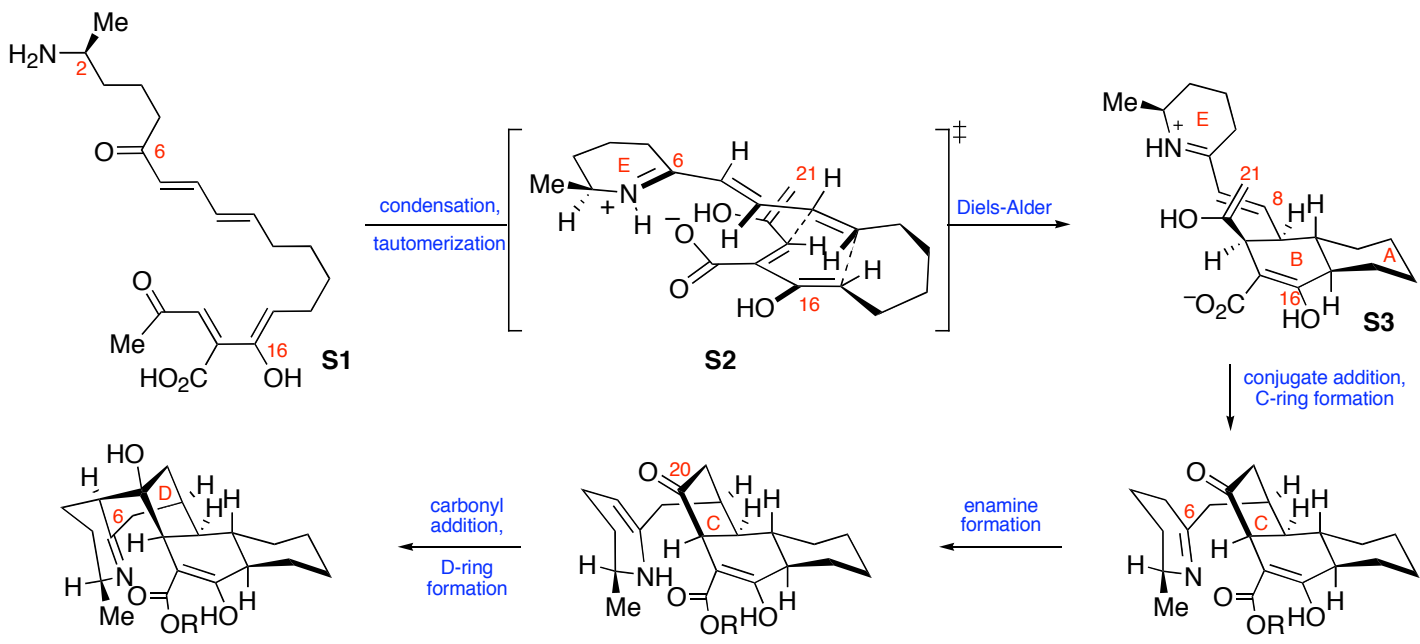

S6, $\mathrm{R}=\mathrm{H}$ or $\mathrm{Me}$

S5, $\mathrm{R}=\mathrm{H}$ or $\mathrm{Me}$

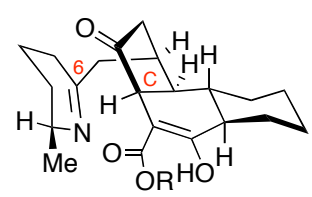

$\downarrow \begin{aligned} & \text { imine } \\ & \text { reduction }\end{aligned}$
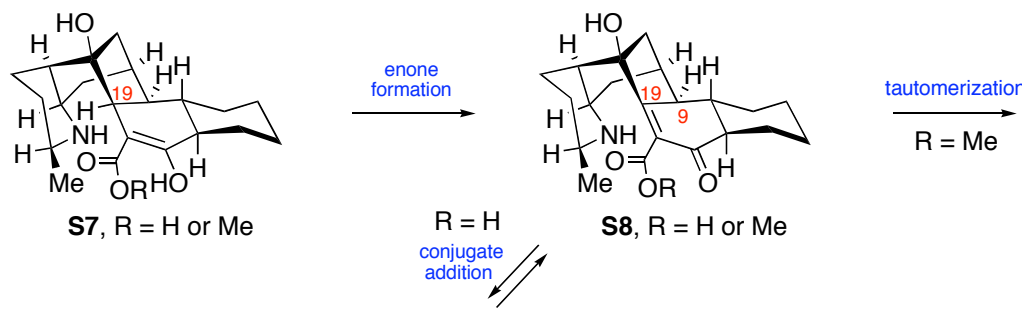

S4, $\mathrm{R}=\mathrm{H}$ or $\mathrm{Me}$

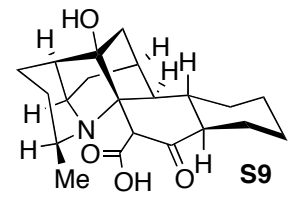

S8, $\mathrm{R}=\mathrm{H}$ or $\mathrm{Me}$

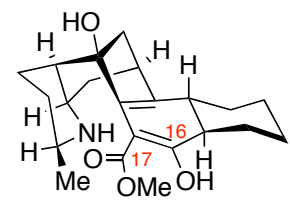

$\mathrm{S12}$
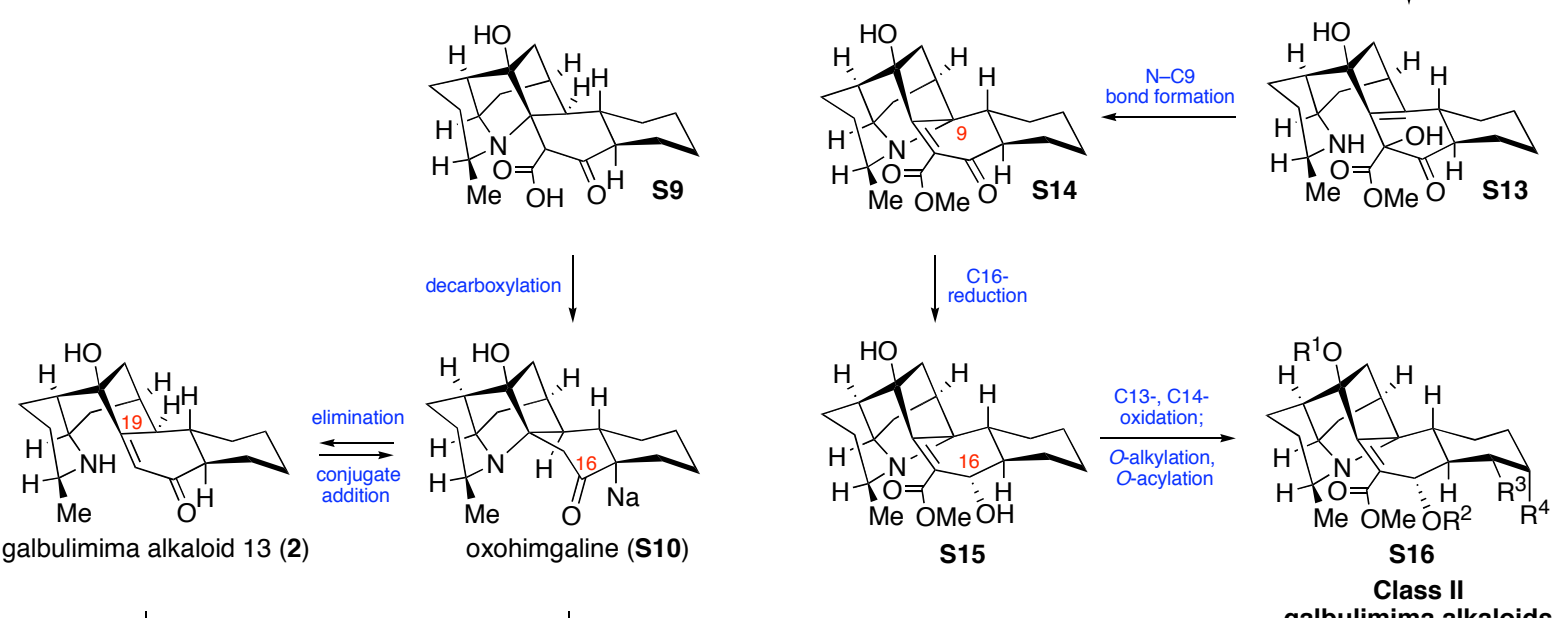

galbulimima alkaloids
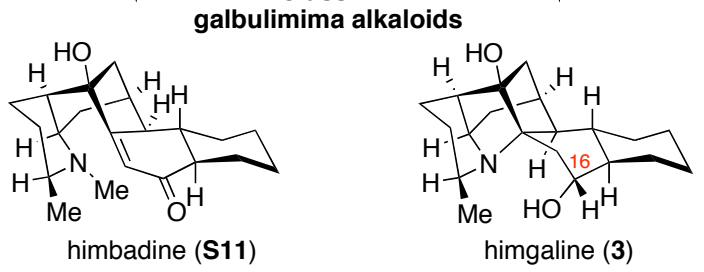


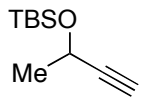

$( \pm)-S 17$

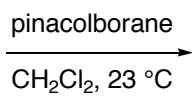

$68 \%$

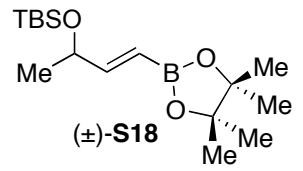

(士)-trans-2-[3-(tert-Butyl-dimethyl-silanyloxy)-but-1-enyl]-4,4,5,5-tetramethyl-[1,3,2]dioxaborolane (S18):

Terminal alkyne $\mathbf{S 1 7 ^ { 9 }}$ (4.70 g, $25.5 \mathrm{mmol}$, 1 equiv) was added dropwise via syringe to a solution of freshly prepared pinacolborane ${ }^{10}$ in dichloromethane $(5 \mathrm{M}, 10 \mathrm{~mL}, 50.2 \mathrm{mmol}, 2.00$ equiv) at $0^{\circ} \mathrm{C}$. The solution was stirred and allowed to warm to ambient temperature. After $24 \mathrm{~h}$, the solution was partitioned between diethyl ether $(300 \mathrm{~mL})$ and saturated aqueous ammonium chloride solution $(150 \mathrm{~mL})$. The aqueous phase was extracted with diethyl ether $(2 \times 150 \mathrm{~mL})$ and the combined organic phases were washed with saturated aqueous ammonium chloride solution (100 $\mathrm{mL})$, were washed with brine $(80 \mathrm{~mL})$, were dried over anhydrous sodium sulfate, were filtered, and were concentrated under reduced pressure. Purification of the resulting oil by flash column chromatography (silica gel: diam. $9 \mathrm{~cm}$, ht. $10 \mathrm{~cm}$; eluent: hexanes:EtOAc [95:5] to hexanes:EtOAc [80:20]) provided boronate $( \pm)-\mathbf{S 1 8}(5.40 \mathrm{~g}, 68 \%)$ as a colorless oil.

${ }^{1} \mathrm{H}$ NMR $\left(500 \mathrm{MHz}, \mathrm{CDCl}_{3}, 20^{\circ} \mathrm{C}\right): \quad 6.60(\mathrm{dd}, J=18,4.0 \mathrm{~Hz}, 1 \mathrm{H}, \mathrm{CH}=\mathrm{CHB}), 5.63(\mathrm{dd}, J=$ $18,1.7 \mathrm{~Hz}, 1 \mathrm{H}, \mathrm{CH}=\mathrm{CHB}), 4.37-4.32(\mathrm{~m}, 1 \mathrm{H}$, $\left.\mathrm{CH}_{3} \mathrm{CHCH}=\mathrm{CH}\right), 1.28\left(\mathrm{~s}, 6 \mathrm{H}, \mathrm{BOC}\left(\mathrm{CH}_{3}\right) \mathrm{CH}_{3}\right), 1.28(\mathrm{~s}$, $\left.6 \mathrm{H}, \mathrm{BOC}\left(\mathrm{CH}_{3}\right) \mathrm{CH}_{3}\right), 1.22\left(\mathrm{~d}, J=6.7 \mathrm{~Hz}, 3 \mathrm{H}, \mathrm{CHCH}_{3}\right)$, $0.91\left(\mathrm{~s}, 9 \mathrm{H}, \mathrm{SiC}\left(\mathrm{CH}_{3}\right)_{3}\right), 0.05\left(\mathrm{~s}, 6 \mathrm{H}, \mathrm{Si}\left(\mathrm{CH}_{3}\right)_{2}\right)$.

${ }^{13} \mathrm{C}$ NMR $\left(125 \mathrm{MHz}, \mathrm{CDCl}_{3}, 20^{\circ} \mathrm{C}\right)$ :

FTIR (thin film) $\mathrm{cm}^{-1}$ :

HRMS (ESI)

$\mathrm{GC}, t_{\mathrm{R}}$ :

TLC (20\% EtOAc in hexanes), Rf:
$157.2(\mathrm{BC}=\mathrm{C}), 83.3(\mathrm{BC}=\mathrm{C}), 70.0\left((\mathrm{Me})_{2} \mathrm{C}\right), 26.1,25.0$, $24.9,23.9,18.5,-4.5\left(\mathrm{SiCH}_{3}\right),-4.6\left(\mathrm{SiCH}_{3}\right)$.

$2929(\mathrm{~m}), 1996(\mathrm{w}), 1611(\mathrm{w}), 1370(\mathrm{w}), 1337(\mathrm{w}), 1146$ (w).

calcd for $\mathrm{C}_{16} \mathrm{H}_{33} \mathrm{BNaO}_{3} \mathrm{Si}[\mathrm{M}+\mathrm{Na}]^{+}:$335.2184, found: 335.2177.

$11.73 \mathrm{~min}$

$0.63\left(\mathrm{KMnO}_{4}\right)$

\footnotetext{
${ }^{9}$ Prepared from 3-butynol, tert-butyldimethylsilylchloride, imidazole, dimethylformamide, $23{ }^{\circ} \mathrm{C}, 12 \mathrm{~h}$; see: Cotterill, A. S.; Gill, M.; Gimenez, A.; Milanovic N. M. J. Chem. Soc., Perkin Trans. 1 1994, 22, 3269.

${ }^{10}$ Pinacolborane was prepared according to Tucker, C. E.; Davidson, J.; Knochel, P. J. Org. Chem. 1992, 57, 3482.
} 


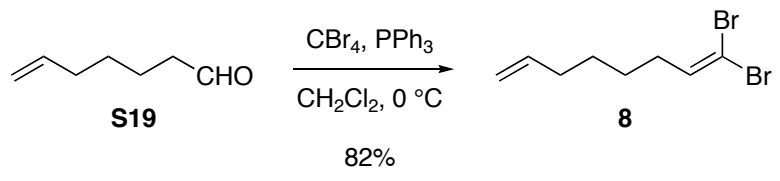

\section{1,1-Dibromo-octa-1,7-diene (8):}

Triphenylphosphine (6.34 g, $24.2 \mathrm{mmol}, 2.40$ equiv) was added in three portions to a solution of carbon tetrabromide $\left(4.00 \mathrm{~g}, 12.1 \mathrm{mmol}, 1.20\right.$ equiv) in dichloromethane $(30 \mathrm{~mL})$ at $0^{\circ} \mathrm{C}$ in an ice bath to produce a yellow-orange solution. The solution was stirred at $0{ }^{\circ} \mathrm{C}$ for $10 \mathrm{~min}$. A solution of aldehyde $\mathbf{S 1 9}^{11}$ (1.12 g, $10.0 \mathrm{mmol}, 1$ equiv) in dichloromethane $(6 \mathrm{~mL})$ was introduced via cannula to the cold reaction mixture. The transfer was completed using a second 4-mL portion of dichloromethane and the mixture was vigorously stirred at $0^{\circ} \mathrm{C}$. The solution became dark orange and white solid precipitated. After $1 \mathrm{~h}$, excess dibromophosphorane was quenched by sequential addition of triethylamine ( $3.4 \mathrm{~mL}, 24 \mathrm{mmol}, 2.4$ equiv) and methanol ( $1.0 \mathrm{~mL}, 25 \mathrm{mmol}, 2.5$ equiv). The solution was allowed to warm to room temperature, transferred to a separatory funnel and added dropwise to a solution of "pentane-diethyl ether $(5: 1,300 \mathrm{~mL})$, resulting in precipitation of triphenylphosphine oxide. The resulting light brown solid was removed by filtration and washed with "pentane $(100 \mathrm{~mL})$. The combined organic filtrate was concentrated and purified by flash column chromatography (silica gel: diam. $5 \mathrm{~cm}$, ht. $10 \mathrm{~cm}$; eluent: hexanes:EtOAc [90:10]) to yield dibromide $\mathbf{8}$ as a colorless oil $(2.21 \mathrm{~g}, 82 \%)$.

$\left.{ }^{1} \mathrm{H} \mathrm{NMR} \mathrm{(500} \mathrm{MHz,} \mathrm{CDCl}_{3}, 20^{\circ} \mathrm{C}\right): \quad 6.40\left(\mathrm{t}, J=7.4 \mathrm{~Hz}, 1 \mathrm{H}, \mathrm{Br}_{2} \mathrm{C}=\mathrm{CH}\right), 5.85-5.76(\mathrm{~m}, 1 \mathrm{H}$, $\left.\mathbf{H C}=\mathrm{CH}_{2}\right), 5.02($ app-dq, $J=17,1.5 \mathrm{~Hz}, 1 \mathrm{H}$, trans$\left.\mathrm{HC}=\mathrm{CH}_{2}\right), 4.97\left(\mathrm{~m}, 1 \mathrm{H}\right.$, cis $\left.-\mathrm{HC}=\mathrm{CH}_{2}\right), 2.14-2.05(\mathrm{~m}$, $\left.4 \mathrm{H}, \mathrm{Br}_{2} \mathrm{C}=\mathrm{CHCH}_{2}, \mathrm{H}_{2} \mathrm{C}=\mathrm{CHCH}_{2}\right), 1.47-1.41(\mathrm{~m}, 4 \mathrm{H}$, $\left.\mathrm{CH}_{2}\left(\mathrm{CH}_{2}\right)_{2} \mathrm{CH}_{2}\right)$.

${ }^{13} \mathrm{C}$ NMR $\left(125 \mathrm{MHz}, \mathrm{CDCl}_{3}, 20^{\circ} \mathrm{C}\right)$ :

FTIR (thin film) $\mathrm{cm}^{-1}$ :

HRMS-EI $(m / z)$ :

$\mathrm{GC}, t_{\mathrm{R}}$ :

TLC (40\% EtOAc in hexanes), $R f$ :
138.9, 138.7, 114.9 $\left(\mathrm{HC}=\mathrm{CH}_{2}\right), 88.9\left(\mathrm{Br}_{2} \mathbf{C}=\mathrm{CH}\right), 33.6$, 33.0, 28.4, 27.4 .

2928 (s), 2857 (m), 1641 (m), 911 (s), 804 (m), 780 (m).

calcd for $\mathrm{C}_{8} \mathrm{H}_{13} \mathrm{Br}_{2}[\mathrm{M}+\mathrm{H}]^{+}$: 265.9300,

found: 265.9324 .

$10.16 \mathrm{~min}$

S19, $0.64\left(\mathrm{KMnO}_{4}\right)$

8, $0.75\left(\mathrm{UV}, \mathrm{KMnO}_{4}\right)$

\footnotetext{
${ }^{11}$ 6-Heptenal was prepared from 7-octene-1,2-diol (commercially available), sodium metaperiodate, diethyl ether, water, $1 \mathrm{~h}, 93 \%$. Spectroscopic data matched published data; see: Taylor, R. E.; Galvin, G. M.; Hilfiker, K. A.; Chen, Y. J. Org. Chem. 1998, 63, 9580.
} 


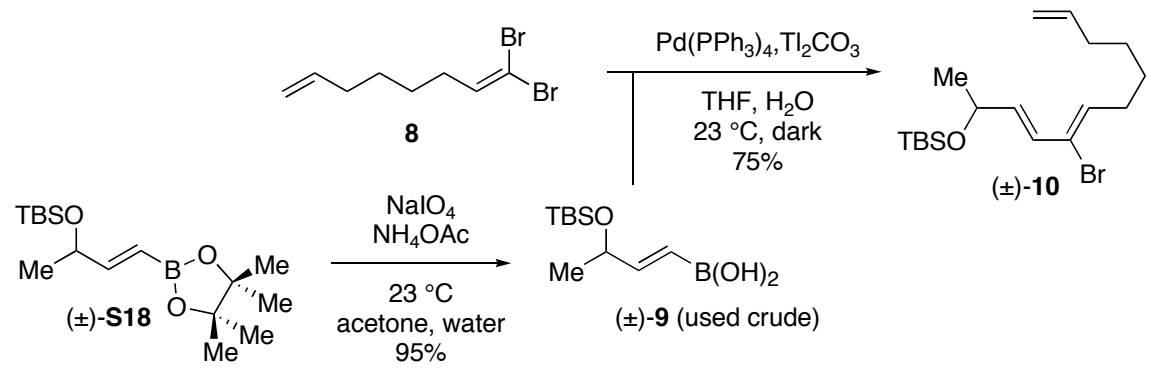

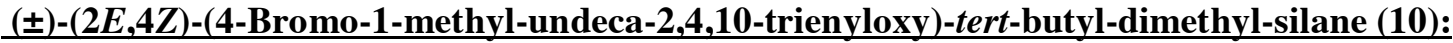

To a solution of boronate $( \pm)-\mathbf{S 1 8}(0.94 \mathrm{~g}, 3.0 \mathrm{mmol}, 1$ equiv) in acetone and water $(30 \mathrm{~mL}$, 2:1) was added sodium metaperiodate $(2.0 \mathrm{~g}, 9.4 \mathrm{mmol}, 3.1 \mathrm{equiv})$ and ammonium acetate $(0.71 \mathrm{~g}$, $9.2 \mathrm{mmol}, 3.0$ equiv). The resulting cloudy solution was stirred at ambient temperature. After $48 \mathrm{~h}$, the reaction mixture was placed under reduced pressure to remove acetone, was diluted with ethyl acetate $(100 \mathrm{~mL})$ and the phases separated. The aqueous layer was extracted with ethyl acetate (100 $\mathrm{mL})$ and the combined organic layers were washed with brine $(50 \mathrm{~mL})$, were dried over anhydrous sodium sulfate, were filtered, and were concentrated under reduced pressure to provide boronic acid $( \pm)-9$ as a light brown oil $(0.66 \mathrm{~g}, 95 \%)$. Dibromide 8 (150 mg, $0.56 \mathrm{mmol}, 1$ equiv) and crude boronic acid $( \pm)-9(160 \mathrm{mg}, 0.69 \mathrm{mmol}, 1.2$ equiv) were combined, dissolved in THF-water (3:1, 11 $\mathrm{mL}$ ), and the solution was degassed thoroughly (FPT). Tetrakis(triphenylphosphine)palladium (33 $\mathrm{mg}, 0.028 \mathrm{mmol}, 0.050$ equiv) was added as a solid, light was excluded, and the resulting clear yellow solution was stirred for $5 \mathrm{~min}$. Thallium carbonate $(0.53 \mathrm{~g}, 1.1 \mathrm{mmol}, 2.0$ equiv) was added as a solid, and the resulting heterogeneous yellow-white mixture was stirred in the dark. After $25 \mathrm{~h}$, the light tan reaction mixture was diluted with ethyl acetate and passed through a silica plug and the clear solution was concentrated. The resulting brown oil was purified by flash column chromatography (silica gel: diam. $2.5 \mathrm{~cm}$, ht. $4 \mathrm{~cm}$; eluent: hexanes:EtOAc [98:2] to hexanes:EtOAc [96:4]) to provide triene $( \pm)-\mathbf{1 0}$ as a yellow oil $(156 \mathrm{mg}, 75 \%)$.

${ }^{1} \mathrm{H}$ NMR $\left(500 \mathrm{MHz}, \mathrm{CDCl}_{3}, 20^{\circ} \mathrm{C}\right): \quad 6.19(\mathrm{~d}, J=14.6 \mathrm{~Hz}, 1 \mathrm{H}, \mathrm{BrCCH}=\mathrm{CH}), 6.03(\mathrm{dd}, J=$ 14.6, $4.8 \mathrm{~Hz}, 1 \mathrm{H}, \mathrm{BrCCH}=\mathrm{CH}), 5.91-5.75(\mathrm{~m}, 2 \mathrm{H}$,

$\left.\mathrm{BrC}=\mathrm{CH} ; \mathrm{CH}_{2}=\mathrm{CH}\right), 5.06-4.93\left(\mathrm{~m}, 2 \mathrm{H}, \mathrm{CH}_{2}=\mathrm{CH}\right), 4.44$ $(\mathrm{m}, 1 \mathrm{H}, \mathrm{TBSOCHCH}), 2.35-2.28(\mathrm{~m}, 2 \mathrm{H}$, $\left.\mathrm{BrC}=\mathrm{CHCH}_{2}\right), 2.10-2.06\left(\mathrm{~m}, 2 \mathrm{H}, \mathrm{CH}_{2}=\mathrm{CHCH}_{2}\right), 1.49$ $1.43\left(\mathrm{~m}, 4 \mathrm{H},\left(\mathrm{CH}_{2}\right)_{2}\right), 1.25\left(\mathrm{~d}, J=6.4 \mathrm{~Hz}, 3 \mathrm{H}, \mathrm{CH}_{3}\right)$, 0.92 (s, 9H, $\left.\operatorname{SiC}\left(\mathrm{CH}_{3}\right)_{3}\right), 0.08\left(\mathrm{~s}, 3 \mathrm{H}, \mathrm{SiCH}_{3}\right), 0.07$ (s, $\left.3 \mathrm{H}, \mathrm{SiCH}_{3}\right)$.

${ }^{13} \mathrm{C}$ NMR $\left(125 \mathrm{MHz}, \mathrm{CDCl}_{3}, 20^{\circ} \mathrm{C}\right)$ :

FTIR (thin film) $\mathrm{cm}^{-1}$ :

HRMS (ESI):

TLC (40\% EtOAc in hexanes), $R f$ :
$138.9,138.3,133.7,127.5,125.2,114.7,68.5$

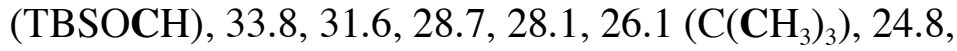
$18.5,-4.4\left(\mathrm{SiCH}_{3}\right),-4.5\left(\mathrm{SiCH}_{3}\right)$.

2955 (s), 2929 (s), 2857 (s), 1472 (w), 1462 (w), 1255

(m), 1149 (m), 1089 (m), 835 (s), 776 (s).

calcd for $\mathrm{C}_{18} \mathrm{H}_{33} \mathrm{NaBrOSi}[\mathrm{M}+\mathrm{Na}]^{+}$: 395.1376 , found: 395.1365 .

9, $0.26\left(\mathrm{KMnO}_{4}\right)$

10, 0.83 (UV, KMnO4) 


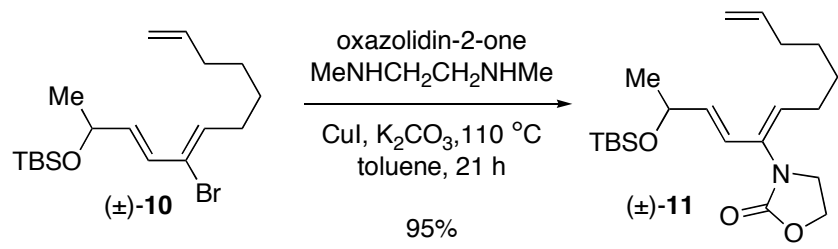

(士)-3-\{(1Z)-1-[(E)-3-(tert-Butyl-dimethyl-silanyloxy)-but-1-enyl]-octa-1,7-dienyl\}-oxazolidin-2-one (11):

Vinyl bromide $( \pm)-\mathbf{1 0}(3.10 \mathrm{~g}, 8.30 \mathrm{mmol}, 1$ equiv) was transferred in dry toluene to a flamedried Schlenk pressure vessel, the solvent was removed under reduced pressure, and the vessel filled with argon. Oxazolidin-2-one (869 mg, $9.96 \mathrm{mmol}, 1.20$ equiv), copper iodide (790 mg, $4.15 \mathrm{mmol}$, 0.500 equiv), and potassium carbonate (2.29 g, $16.6 \mathrm{mmol}, 2.00$ equiv) were added under argon, and the vessel was evacuated and back-filled with argon three times. Dimethylethylenediamine $(2.23$ $\mathrm{mL}, 20.8 \mathrm{mmol}, 2.50$ equiv) and toluene $(33 \mathrm{~mL})$ were added. The reaction vessel was sealed under argon atmosphere and the green-gray heterogeneous mixture was heated to $110{ }^{\circ} \mathrm{C}$. The solution turned slate-blue after five minutes, then light yellow-green. After $21 \mathrm{~h}$, the solution was cooled to ambient temperature and partitioned between ethyl acetate $(200 \mathrm{~mL})$ and water $(100 \mathrm{~mL})$. The blue aqueous layer was extracted with ethyl acetate $(3 \times 150 \mathrm{~mL})$, and the combined yellow organic layers were washed with brine $(50 \mathrm{~mL})$, were dried over anhydrous sodium sulfate, were filtered, and were concentrated under reduced pressure. The resulting brown oil was purified by flash column chromatography (silica gel: diam. $7 \mathrm{~cm}$, ht. $10 \mathrm{~cm}$; eluent: $\mathrm{CH}_{2} \mathrm{Cl}_{2}$ :acetone [99:1] to $\mathrm{CH}_{2} \mathrm{Cl}_{2}$ :acetone [96:4] to $\mathrm{CH}_{2} \mathrm{Cl}_{2}$ :acetone [85:15]) to provide triene ( \pm )-11 as a light yellow oil (2.98 $\left.\mathrm{g}, 95 \%\right)$.

${ }^{1} \mathrm{H}$ NMR (500 MHz, $\left.\mathrm{C}_{6} \mathrm{D}_{6}, 20^{\circ} \mathrm{C}\right)$ :

${ }^{13} \mathrm{C}$ NMR $\left(125 \mathrm{MHz}, \mathrm{C}_{6} \mathrm{D}_{6}, 20^{\circ} \mathrm{C}\right)$ :

FTIR (thin film) $\mathrm{cm}^{-1}$ :

HRMS (ESI):

TLC ( $3 \%$ acetone in $\left.\mathrm{CH}_{2} \mathrm{Cl}_{2}\right), R f$ : $6.09(\mathrm{dd}, J=15.4,1.0 \mathrm{~Hz}, 1 \mathrm{H},(\mathrm{TBSO}) \mathrm{CHCH}=\mathrm{CH})$, 5.77-5.69 (m, 1H, CH= $\left.\mathrm{CH}_{2}\right), 5.63(\mathrm{dd}, J=15.6,5.5 \mathrm{~Hz}$, $1 \mathrm{H},(\mathrm{TBSO}) \mathrm{CHCH}=\mathrm{CH}), 5.46(\mathrm{t}, J=7.4 \mathrm{~Hz}, 1 \mathrm{H}$, $(\mathrm{N}) \mathrm{C}=\mathrm{CH}), 5.04-4.97\left(\mathrm{~m}, 2 \mathrm{H}, \mathrm{CH}=\mathrm{CH}_{2}\right), 4.27$ (app-p, $J$ $=6.1 \mathrm{~Hz}, 1 \mathrm{H},(\mathrm{TBSO}) \mathrm{CH}), 3.55-3.51(\mathrm{~m}, 2 \mathrm{H}$, $\left.\mathrm{OCH}_{2} \mathrm{CH}_{2} \mathrm{~N}\right), 3.02-2.91\left(\mathrm{~m}, 2 \mathrm{H}, \mathrm{OCH}_{2} \mathrm{CH}_{2} \mathrm{~N}\right)$, 2.04$1.98\left(\mathrm{~m}, 2 \mathrm{H}, \mathrm{CH}_{2} \mathrm{CH}=\mathrm{CH}_{2}\right), 1.94-1.90(\mathrm{~m}, 2 \mathrm{H}$, $\left.(\mathrm{N}) \mathrm{C}=\mathrm{CHCH}_{2}\right), 1.28-1.24\left(\mathrm{~m}, 4 \mathrm{H},\left(\mathrm{CH}_{2}\right)_{2}\right), 1.21(\mathrm{~d}, J=$ $6.3 \mathrm{~Hz}, 3 \mathrm{H}, \mathrm{TBSOCHCH})_{3}, 1.01\left(\mathrm{~s}, 9 \mathrm{H}, \mathrm{SiC}\left(\mathrm{CH}_{3}\right)_{3}\right)$, 0.11 (s, 3H, $\left.\mathrm{Si}\left(\mathrm{CH}_{3}\right)_{2}\right), 0.10$ (s, 3H, $\left.\mathrm{Si}\left(\mathrm{CH}_{3}\right)_{2}\right)$.

$156.2(\mathrm{O}=\mathrm{C}), 139.2,134.5,134.1,134.0,125.6,115.1$, 69.5, 61.9, 46.2, 34.3, 29.3, 28.8, 28.3, 26.5, $\left(\mathrm{C}\left(\mathrm{CH}_{3}\right)_{3}\right)$, $25.2,18.8,-4.0\left(\mathrm{SiCH}_{3}\right),-4.2\left(\mathrm{SiCH}_{3}\right)$.

$2928(w), 2856(w), 1758(\mathrm{~s}), 1414(\mathrm{~m}), 1251(\mathrm{w}), 834$ (m).

calcd for $\mathrm{C}_{21} \mathrm{H}_{37} \mathrm{NaNO}_{3} \mathrm{Si}[\mathrm{M}+\mathrm{Na}]^{+}$: 402.2435, found: 402.2444 .

10, 0.89 (UV, CAM)

11, 0.54 (UV, CAM) 


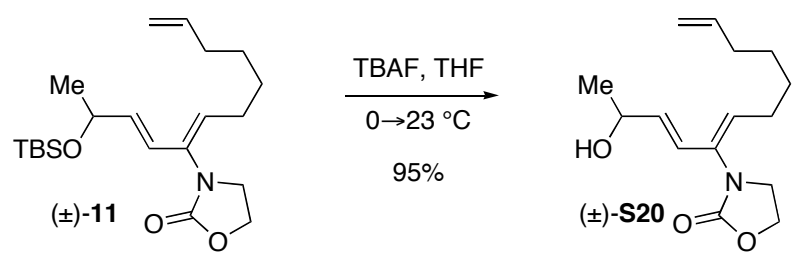

\section{(士)-3-[(1Z)-1-((E)-3-Hydroxy-but-1-enyl)-octa-1,7-dienyl]-oxazolidin-2-one (S20):}

A solution of tetrabutylammonium fluoride in THF (1M, $1.9 \mathrm{~mL}, 1.9 \mathrm{mmol}, 1.5$ equiv) was added to a solution of silyl ether $( \pm)-11\left(487 \mathrm{mg}, 1.28 \mathrm{mmol}, 1\right.$ equiv) in THF $(10 \mathrm{~mL})$ at $0{ }^{\circ} \mathrm{C}$. The resulting light yellow solution was vigourously stirred and allowed to warm to ambient temperature. After $3.5 \mathrm{~h}$, the reaction mixture was diluted with ethyl acetate $(50 \mathrm{~mL})$, water $(5 \mathrm{~mL})$, and saturated aqueous ammonium chloride solution $(25 \mathrm{~mL})$. The aqueous layer was extracted with ethyl acetate $(3 \times 50 \mathrm{ml})$, and the combined organic layers were washed with brine $(25 \mathrm{~mL})$, were dried over anhydrous sodium sulfate, were filtered, and were concentrated under reduced pressure. The resulting yellow oil was purified by flash column chromatography (silica gel: diam. $2.5 \mathrm{~cm}$, ht. 6.5 cm; eluent: $\mathrm{CH}_{2} \mathrm{Cl}_{2}$ :acetone [95:5] to $\mathrm{CH}_{2} \mathrm{Cl}_{2}$ :acetone [80:20] to $\mathrm{CH}_{2} \mathrm{Cl}_{2}$ :acetone [50:50]) to provide the alcohol $( \pm)-\mathbf{S 2 0}$ as a clear oil $(321 \mathrm{mg}, 95 \%)$.

${ }^{1} \mathrm{H}$ NMR (500 MHz, $\left.\mathrm{C}_{6} \mathrm{D}_{6}, 20^{\circ} \mathrm{C}\right)$ :

${ }^{13} \mathrm{C}$ NMR $\left(125 \mathrm{MHz}, \mathrm{C}_{6} \mathrm{D}_{6}, 20^{\circ} \mathrm{C}\right)$ :

FTIR (thin film) $\mathrm{cm}^{-1}$ :

HRMS (ESI):

TLC (10\% acetone in $\left.\mathrm{CH}_{2} \mathrm{Cl}_{2}\right), R f$ : $5.99(\mathrm{~d}, J=15.6 \mathrm{~Hz}, 1 \mathrm{H}, \mathrm{MeC}(\mathrm{OH}) \mathrm{CH}=\mathrm{CH}), 5.81-5.71$ $\left(\mathrm{m}, 1 \mathrm{H}, \mathrm{CH}=\mathrm{CH}_{2}\right), 5.64(\mathrm{dd}, J=15.6,5.5 \mathrm{~Hz}, 1 \mathrm{H}$, $\mathrm{MeC}(\mathrm{OH}) \mathrm{CH}=\mathrm{CH}), 5.41(\mathrm{t}, J=7.4 \mathrm{~Hz}, 1 \mathrm{H},(\mathrm{N}) \mathrm{C}=\mathrm{CH})$, 5.07-4.97 (m, 2H, CH=CH CH $_{2} 4.24$ (br-s, $\left.1 \mathrm{H}, \mathrm{CHOH}\right)$, 3.67-3.57 (m, 2H, $\left.\mathrm{OCH}_{2} \mathrm{CH}_{2} \mathrm{~N}\right), 2.97(\mathrm{t}, J=8.0 \mathrm{~Hz}, 2 \mathrm{H}$, $\mathrm{OCH}_{2} \mathrm{CH}_{2} \mathrm{~N}$ ), 2.36-2.18 (br-s, OH), 2.03-1.91 (m, 4H, (allylic $\left.\mathrm{CH}_{2}\right), 1.32-1.25\left(\mathrm{~m}, 4 \mathrm{H},\left(\mathrm{CH}_{2}\right)_{2}, 1.23(\mathrm{~d}, J=6.3\right.$ $\mathrm{Hz}, 3 \mathrm{H}, \mathrm{CH}_{3}$ ).

$156.7(\mathrm{O}=\mathrm{C}), 139.2(\mathrm{C}=\mathrm{CN}), 134.5,134.1,128.7,126.1$, $115.1\left(\mathrm{HC}=\mathrm{CH}_{2}\right), 68.3,62.2,46.2,34.3,29.3,28.9$, 28.2, 24.0.

3421 (br-m, OH), 2973 (w), 2927 (m), 2857 (w), 1741 (s, C=O), 1419 (s), 1247 (m), 1037 (m).

calcd for $\mathrm{C}_{15} \mathrm{H}_{23} \mathrm{NaNO}_{3}[\mathrm{M}+\mathrm{Na}]^{+}:$288.1572, found: 288.1572 .

11, 0.75 (UV, CAM)

S20, 0.16 (UV, CAM) 


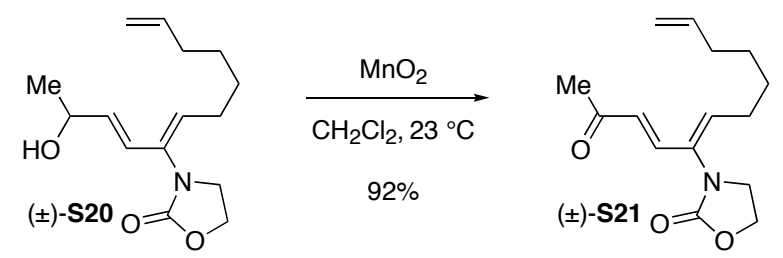

(土)-3-[(1Z)-1-((E)-3-Oxo-but-1-enyl)-octa-1,7-dienyl]-oxazolidin-2-one (S20):

$\gamma$-Manganese dioxide (1.21 g, $13.9 \mathrm{mmol}, 11.7$ equiv) was added under an argon atmosphere in one portion to a solution of alcohol $( \pm)$-S20 $(317 \mathrm{mg}, 1.20 \mathrm{mmol}, 1$ equiv) in dichloromethane (6 $\mathrm{mL}$ ) and the mixture was stirred at ambient temperature. After $19.5 \mathrm{~h}$, the reaction mixture was diluted with dichloromethane and passed through celite. The resulting solution was concentrated under reduced pressure to provide spectroscopically clean ketone $( \pm)$-S21 as a clear oil (289 $\mathrm{mg}$, $92 \%$ ). If desired, purification of ketone ( \pm )-S21 could be achieved via flash column chromatography (silica gel, eluent: $\mathrm{CH}_{2} \mathrm{Cl}_{2}$ :acetone [98:2] to $\mathrm{CH}_{2} \mathrm{Cl}_{2}$ :acetone [90:10]).

${ }^{1} \mathrm{H}$ NMR $\left(500 \mathrm{MHz}, \mathrm{C}_{6} \mathrm{D}_{6}, 20^{\circ} \mathrm{C}\right)$ :

${ }^{13} \mathrm{C}$ NMR $\left(125 \mathrm{MHz}, \mathrm{C}_{6} \mathrm{D}_{6}, 20^{\circ} \mathrm{C}\right)$ :

FTIR (thin film) $\mathrm{cm}^{-1}$ :

HRMS (ESI):

TLC (10\% acetone in $\mathrm{CH}_{2} \mathrm{Cl}_{2}$ ), Rf: $6.77(\mathrm{~d}, J=15.8 \mathrm{~Hz}, 1 \mathrm{H}, \mathrm{MeCOCH}=\mathrm{CH}), 6.00(\mathrm{~d}, J=$ $15.8 \mathrm{~Hz}, 1 \mathrm{H}, \mathrm{MeCOCH}=\mathrm{CH}), 5.78-5.70(\mathrm{~m}, 1 \mathrm{H}$, $\left.\mathrm{CH}=\mathrm{CH}_{2}\right), 5.52(\mathrm{t}, J=7.6 \mathrm{~Hz}, 1 \mathrm{H},(\mathrm{N}) \mathrm{C}=\mathrm{CH}), 5.06-4.99$ $\left(\mathrm{m}, 2 \mathrm{H}, \mathrm{CH}=\mathrm{CH}_{2}\right.$ ), 3.47 (app-t, $J=7.8 \mathrm{~Hz}, 2 \mathrm{H}$, $\mathrm{OCH}_{2} \mathrm{CH}_{2} \mathrm{~N}$ ), 2.73 (app-t, $J=7.8 \mathrm{~Hz}, 2 \mathrm{H}, \mathrm{OCH}_{2} \mathrm{CH}_{2} \mathrm{~N}$ ), 1.94-1.90 (m, 7H, allylic-C $\mathbf{H}_{2}$, allylic- $\left.\mathrm{CH}_{2}, \mathrm{Me}\right), 1.21$ $1.20\left(\mathrm{~m}, 4 \mathrm{H},\left(\mathrm{CH}_{2}\right)_{2}\right)$.

196.6 (ketone-C=O), 156.1 (carbamate- $\mathrm{C}=\mathrm{O}), 143.7$, $140.2,139.0,134.1,126.6,115.3,62.2\left(\mathrm{OCH}_{2} \mathrm{CH}_{2} \mathrm{~N}\right)$, $46.0\left(\mathrm{OCH}_{2} \mathrm{CH}_{2} \mathrm{~N}\right), 34.2,29.3,28.8,28.3,27.9$.

2924 (m), 1754 (s, C=O), 1746 (s, C=O), 1666 (m), 1631 (m), 1599 (m), 1414 (s), 1251 (m).

calcd for $\mathrm{C}_{15} \mathrm{H}_{21} \mathrm{NaNO}_{3}[\mathrm{M}+\mathrm{Na}]^{+}:$: 286.1414, found: 286.1421 .

S20, 0.16 (UV, CAM)

S21, 0.42 (UV, CAM) 

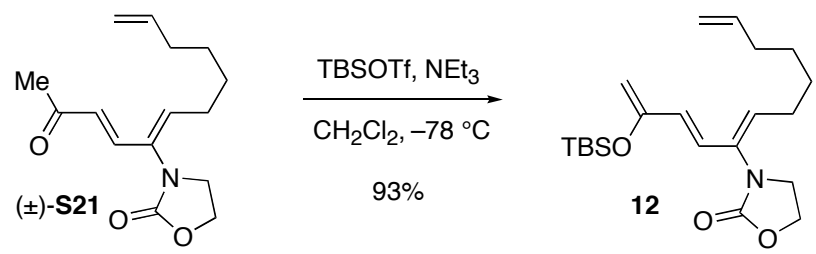

\section{3-\{(1Z)-1-[(Z)-3-(tert-Butyl-dimethyl-silanyloxy)-but-1,3 dienyl]-octa-1,7-dienyl\}-oxazolidin-2-one (12):}

Triethylamine $(860 \mu \mathrm{L}, 6.12 \mathrm{mmol}, 1.50$ equiv) was added to a solution of ketone $( \pm)-\mathbf{S 2 1}$ (1.07 g, $4.08 \mathrm{mmol}, 1$ equiv) in dichloromethane $(20 \mathrm{~mL})$ at $-78{ }^{\circ} \mathrm{C}$, followed by dropwise addition of TBSOTf (1.12 mL, $4.89 \mathrm{mmol}, 1.20$ equiv). After $15 \mathrm{~min}$, the excess silylating agent was quenched by the addition of saturated aqueous sodium bicarbonate solution $(5 \mathrm{~mL})$ and allowed to warm to ambient temperature. The reaction mixture was diluted with ethyl acetate $(80 \mathrm{~mL})$ and washed with saturated aqueous sodium bicarbonate solution $(30 \mathrm{~mL})$. The aqueous phase was extracted with ethyl acetate $(4 \times 75 \mathrm{~mL})$, and the combined organic layers were washed with brine $(20 \mathrm{~mL})$, were dried over anhydrous sodium sulfate, were filtered, and were concentrated under reduced pressure. The resulting oil was purified by flash column chromatography (neutralized silica gel: diam. $5 \mathrm{~cm}$, ht. $9 \mathrm{~cm}$; eluent: $\mathrm{CH}_{2} \mathrm{Cl}_{2}$ :acetone [97:3]) to provide the silyl enol ether $\mathbf{1 2}$ as a white solid $(1.44 \mathrm{~g}, 93 \%)$.

${ }^{1} \mathrm{H}$ NMR $\left(500 \mathrm{MHz}, \mathrm{C}_{6} \mathrm{D}_{6}, 20^{\circ} \mathrm{C}\right)$ :

$6.80(\mathrm{~d}, J=15.3 \mathrm{~Hz}, 1 \mathrm{H}, \mathbf{H C}=\mathrm{CHCOTBS}), 6.06(\mathrm{~d}, J=$ $15.3 \mathrm{~Hz}, 1 \mathrm{H}, \mathrm{HC}=\mathrm{CHCOTBS})$, 5.76-5.68 $(\mathrm{m}, 1 \mathrm{H}$, $\left.\mathrm{CH}=\mathrm{CH}_{2}\right), 5.60(\mathrm{t}, J=7.4 \mathrm{~Hz}, 1 \mathrm{H},(\mathrm{N}) \mathrm{C}=\mathrm{CH}), 5.03-4.96$ ( $\left.\mathrm{m}, 2 \mathrm{H}, \mathrm{CH}=\mathrm{CH}_{2}\right), 4.42$ (s, 1H, $\left.\mathbf{C H}_{2}=\mathrm{CHOTBS}\right), 4.33$ (s, $1 \mathrm{H}, \mathrm{CH}_{2}=\mathrm{CHOTBS}$ ), 3.51 (app-t, $J=7.6 \mathrm{~Hz} .2 \mathrm{H}$, $\mathrm{OCH}_{2} \mathrm{CH}_{2} \mathrm{~N}$ ), 2.89 (app-t, $J=8.0 \mathrm{~Hz}, 2 \mathrm{H}, \mathrm{OCH}_{2} \mathrm{CH}_{2} \mathrm{~N}$ ), 2.03-1.99 (m, 2H, $\left.\mathrm{CH}_{2} \mathrm{CH}=\mathrm{CH}_{2}\right), 1.95-1.85(\mathrm{~m}, 2 \mathrm{H}$, $\left.(\mathrm{N}) \mathrm{C}=\mathrm{CHCH}_{2}\right), 1.24-1.21\left(\mathrm{~m}, 4 \mathrm{H},\left(\mathrm{CH}_{2}\right)_{2}\right), 1.02(\mathrm{~s}, 9 \mathrm{H}$, $\left.\mathrm{SiC}\left(\mathrm{CH}_{3}\right)_{3}\right), 0.18\left(\mathrm{~s}, 6 \mathrm{H}, \mathrm{Si}\left(\mathrm{CH}_{3}\right)_{2}\right)$.

${ }^{13} \mathrm{C}$ NMR $\left(125 \mathrm{MHz}, \mathrm{C}_{6} \mathrm{D}_{6}, 20^{\circ} \mathrm{C}\right)$ :

FTIR (thin film) $\mathrm{cm}^{-1}$ :

HRMS (ESI):
$156.3,155.6,139.2,136.1,134.8,127.3,126.5,115.1$, 97.4, 62.1, 46.3, 34.2, 29.3, 28.7, 28.6, 26.4, 18.9, -4.2.

2930 (s), 2858 (s), 1759 (s, C=O), 1415 (m), 1316 (m), $1254(\mathrm{~m}), 1030(\mathrm{~m}), 840(\mathrm{~m})$.

calcd for $\mathrm{C}_{21} \mathrm{H}_{36} \mathrm{NO}_{3} \mathrm{Si}[\mathrm{M}+\mathrm{H}]^{+}$: 378.2459, found: 378.2465 .

TLC, $R f$ :

( $10 \%$ acetone in $\mathrm{CH}_{2} \mathrm{Cl}_{2}$, neutralized plates): $\mathbf{S 2 1}, 0.58$ (UV, CAM)

$$
\text { 12, } 0.79 \text { (UV, CAM) }
$$



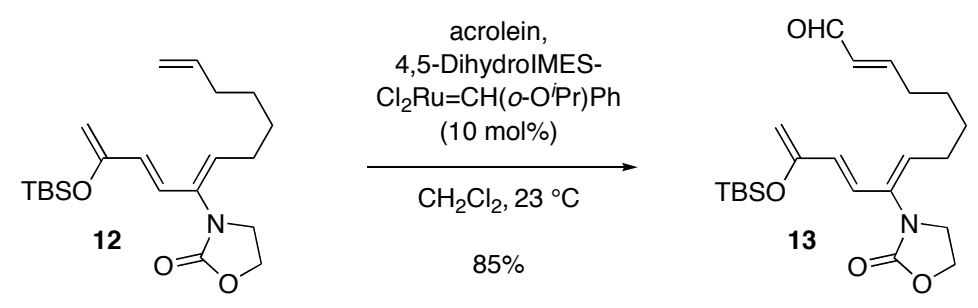

$\underline{(2 E, 8 Z, 10 E)-12-(t e r t-B u t y l-d i m e t h y l-s i l a n y l o x y)-9-(2-o x o-o x a z o l i d i n-3-y l)-t r i d e c a-2,8,10,12-t e t r a e n-1-a l ~}$ (13):

To a solution of silyl enol ether $12(500 \mathrm{mg}, 1.32 \mathrm{mmol}, 1$ equiv) in dichloromethane (6.6 $\mathrm{mL})$ was added freshly distilled acrolein $(354 \mu \mathrm{L}, 5.30 \mathrm{mmol}, 4.00$ equiv, no stabilizer present), followed by 4,5-DihydroIMESCl${ }_{2} \mathrm{Ru}=\mathrm{CH}\left(o-\mathrm{O} \mathrm{Pr}^{\prime} \mathrm{Ph}^{12}(82 \mathrm{mg}, 0.13 \mathrm{mmol}, 0.10\right.$ equiv $)$. The green solution was stirred at ambient temperature for 10 minutes, then purified immediately without concentration via flash column chromatography (neutralized silica gel: diam. $5 \mathrm{~cm}$, ht. $8 \mathrm{~cm}$; eluent: $\mathrm{CH}_{2} \mathrm{Cl}_{2}$ :acetone: $\mathrm{NEt}_{3}$ [98:1:1]) to provide the tetraene 13 as a tan solid (455 $\mathrm{mg}, 85 \%$ ).

${ }^{1} \mathrm{H}$ NMR $\left(500 \mathrm{MHz}, \mathrm{C}_{6} \mathrm{D}_{6}, 20^{\circ} \mathrm{C}\right)$ :

$9.34(\mathrm{~d}, J=7.6 \mathrm{~Hz}, 1 \mathrm{H}, \mathrm{CHO}), 6.71(\mathrm{~d}, J=15.3 \mathrm{~Hz}, 1 \mathrm{H}$, HC=CHCOTBS $) 6.07(\mathrm{~d}, J=15.3 \mathrm{~Hz}, 1 \mathrm{H}$, $\mathrm{HC}=\mathrm{CHCOTBS}), 6.01(\mathrm{dd}, J=15.6,6.6 \mathrm{~Hz}, 1 \mathrm{H}$, $\mathrm{CH}=\mathrm{CHCHO}), 5.90(\mathrm{dd}, J=15.6,7.6 \mathrm{~Hz}, 1 \mathrm{H}$, $\mathrm{CH}=\mathrm{CHCHO}), 5.55(\mathrm{t}, J=7.4 \mathrm{~Hz}, 1 \mathrm{H},(\mathrm{N}) \mathrm{C}=\mathrm{CH}), 4.43$ (s, $\left.1 \mathrm{H}, \mathrm{CH}_{2}=\mathrm{CHOTBS}\right), 4.34$ (s, 1H, $\left.\mathrm{CH}_{2}=\mathrm{CHOTBS}\right)$, 3.55 (app-t, $J=7.9 \mathrm{~Hz}, 2 \mathrm{H}, \mathrm{OCH}_{2} \mathrm{CH}_{2} \mathrm{~N}$ ), 2.90 (app-t, $J$ $\left.=7.4 \mathrm{~Hz}, 2 \mathrm{H}, \mathrm{OCH}_{2} \mathrm{CH}_{2} \mathrm{~N}\right), 1.93($ app-q, $J=7.2 \mathrm{~Hz}, 2 \mathrm{H}$, $\left.\mathrm{CH}_{2} \mathrm{CH}=\mathrm{CH}_{2}\right), 1.67$ (app-q, $J=6.9 \mathrm{~Hz}, 2 \mathrm{H}$, $(\mathrm{N}) \mathrm{C}=\mathrm{CHCH})_{2}, 1.11-0.94\left(\mathrm{~m}, 13 \mathrm{H},\left(\mathrm{CH}_{2}\right)_{2}, \mathrm{SiC}\left(\mathrm{CH}_{3}\right)_{3}\right)$, $0.18\left(\mathrm{~s}, 6 \mathrm{H}, \mathrm{Si}\left(\mathrm{CH}_{3}\right)_{2}\right)$.

${ }^{13} \mathrm{C}$ NMR (125 MHz, $\left.\mathrm{C}_{6} \mathrm{D}_{6}, 20^{\circ} \mathrm{C}\right)$ :

193.2 (CHO), 157.5, 156.4, 155.5, 135.5, 135.0, 133.6, 127.0, 126.8, 97.5, 62.2, 46.3, 32.6, 28.5, 28.3, 27.9, $26.3,18.8,-4.2$.

FTIR (thin film) $\mathrm{cm}^{-1}$ : 2951 (s), 2930 (s), 2858 (m), 1753 (s), 1689 (s), 1414 (m), $1253(\mathrm{~m}), 840(\mathrm{~m})$.

HRMS (ESI):

calcd for $\mathrm{C}_{22} \mathrm{H}_{35} \mathrm{NaNO}_{4} \mathrm{Si}[\mathrm{M}+\mathrm{Na}]^{+}:$428.2228, found: 428.2226 .

TLC, $R f$ :

(3\% acetone in $\mathrm{CH}_{2} \mathrm{Cl}_{2}$, neutralized plates)
12, 0.63 (UV, CAM)
13, 0.30 (UV, CAM)

\footnotetext{
${ }^{12}$ Garber, S. B.; Kingsbury, J. S.; Gray, B. L.; Hoveyda, A. H. J. Am. Chem. Soc. 2000, 122, 8168. Grubbs' G1 and G2 Ru-catalysts required above ambient temperatures found to be incompatible with the sensitive tetraene product $\mathbf{1 3}$.
} 


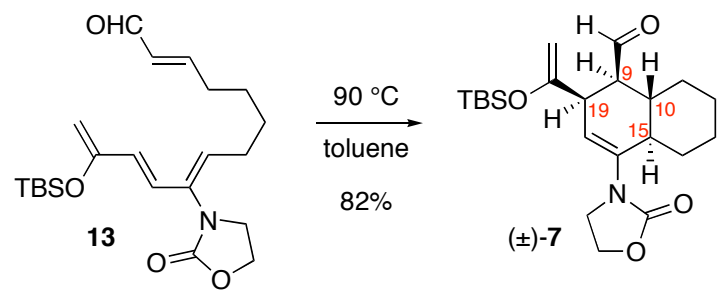

$( \pm)-(9 S, 10 R, 15 R, 19 S)-$ trans-Decalin aldehyde 7:

A flame-dried Schlenk flask was charged with tetraene 13 (279 mg, $0.688 \mathrm{mmol}, 1$ equiv) and toluene $(34 \mathrm{~mL})$ and sealed under argon atmosphere. The vessel was heated to $90{ }^{\circ} \mathrm{C}$. After $13 \mathrm{~h}$, the solvent was removed under reduced pressure and the resulting oil was purified by flash column chromatography (neutralized silica gel: diam. $2.5 \mathrm{~cm}$, ht. $8 \mathrm{~cm}$; eluent: $\mathrm{CH}_{2} \mathrm{Cl}_{2}$ :acetone: $\mathrm{NEt}_{3}$ [94:5:1]) to provide the $( \pm)$-trans-decalin aldehyde 7 as a yellow oil $(228 \mathrm{mg}, 82 \%)$.

${ }^{1} \mathrm{H}$ NMR (500 MHz, $\left.\mathrm{C}_{6} \mathrm{D}_{6}, 20^{\circ} \mathrm{C}\right): \quad 9.66(\mathrm{~d}, J=5.2 \mathrm{~Hz}, 1 \mathrm{H}, \mathrm{CHO}), 5.01(\mathrm{dd}, J=5.2,2.1 \mathrm{~Hz}$, $1 \mathrm{H}, \mathrm{NC}=\mathrm{CH}), 4.37\left(\mathrm{~d}, J=1.1 \mathrm{~Hz}, 1 \mathrm{H}, \mathrm{C}=\mathrm{CH}_{2}\right), 4.34(\mathrm{~d}$, $\left.J=1.0 \mathrm{~Hz}, 1 \mathrm{H}, \mathrm{C}=\mathrm{CH} \mathbf{H}_{2}\right), 3.42-3.33(\mathrm{~m}, 2 \mathrm{H}$, $\mathrm{OCH}_{2} \mathrm{CH}_{2} \mathrm{~N}$ ), 2.98-2.95 (m, 1H, CHCOTBS), 2.79 (q, $J$ $\left.=8.6 \mathrm{~Hz}, 1 \mathrm{H}, \mathrm{OCH}_{2} \mathrm{CH}_{2} \mathrm{~N}\right), 2.50-2.45(\mathrm{~m}, 1 \mathrm{H}$, $\left.\mathrm{OCH}_{2} \mathrm{CH}_{2} \mathrm{~N}\right), 2.33-2.28$ (m, $\left.1 \mathrm{H}, \mathrm{HCC}(\mathrm{N}) \mathrm{CH}\right), 2.26-2.21$ (m, 1H, CHOCH), 2.04-1.97 (m, 2H, CHOCHCH), 1.67-1.62 (m, 2H, $\left.\left(\mathrm{CH}_{2}\right)_{4}\right)$, 1.54-1.52 (m, 1H, $\left.\left(\mathrm{CH}_{2}\right)_{4}\right)$, $1.26\left(\mathrm{qt}, J=13.2,3.8 \mathrm{~Hz}, 1 \mathrm{H},\left(\mathrm{CH}_{2}\right)_{4}\right), 1.17-1.09(\mathrm{~m}$, $\left.1 \mathrm{H},\left(\mathrm{CH}_{2}\right)_{4}\right), 1.03-0.92\left(\mathrm{~m}, 1 \mathrm{H},\left(\mathrm{CH}_{2}\right)_{4}\right), 0.97(\mathrm{~s}, 9 \mathrm{H}$, $\left.\mathrm{SiC}\left(\mathrm{CH}_{3}\right)_{3}\right), 0.83\left(\mathrm{qd}, J=3.8,12.6 \mathrm{~Hz}, 1 \mathrm{H},\left(\mathrm{CH}_{2}\right)_{4}\right), 0.14$ $\left(\mathrm{s}, 3 \mathrm{H}, \mathrm{SiCH}_{3}\right), 0.12\left(\mathrm{~s}, 3 \mathrm{H}, \mathrm{SiCH}_{3}\right)$.

${ }^{13} \mathrm{C}$ NMR $\left(125 \mathrm{MHz}, \mathrm{C}_{6} \mathrm{D}_{6}, 20^{\circ} \mathrm{C}\right)$ :

203.6 (CHO), 158.2 (carbamate-C=O), 156.2, 140.8, 117.8, 95.4, $62.0\left(\mathrm{OCH}_{2} \mathrm{CH}_{2} \mathrm{~N}\right), 53.9,46.7$

$\left(\mathrm{OCH}_{2} \mathrm{CH}_{2} \mathrm{~N}\right), 44.4,42.7,37.4,30.9,27.2,27.0,26.4$ $\left(\mathrm{SiC}\left(\mathrm{CH}_{3}\right)_{3}, 18.8,-3.9\left(\mathrm{SiCH}_{3}\right),-4.3\left(\mathrm{SiCH}_{3}\right)\right.$.

nOe data $\left(500 \mathrm{MHz}, \mathrm{C}_{6} \mathrm{D}_{6}, 20^{\circ} \mathrm{C}\right)$ :

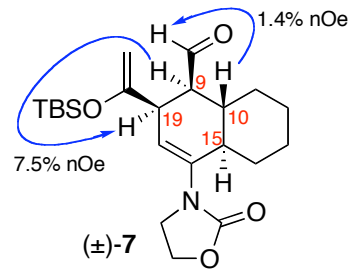

FTIR (thin film) $\mathrm{cm}^{-1}$ : 2929 (s), 2857 (m), 1756 (s), 1724 (s), 1408 (m), 1255 (m), $1220(\mathrm{~m}), 837$ (s).

HRMS (ESI): calcd for $\mathrm{C}_{22} \mathrm{H}_{36} \mathrm{NO}_{4} \mathrm{Si}[\mathrm{M}+\mathrm{H}]^{+}:$406.2408, found: 406.2403 .

TLC, $R f$ :

(30\% acetone in hexanes, neutralized plates) 13, 0.26 (UV, CAM) 7, 0.33 (CAM) 


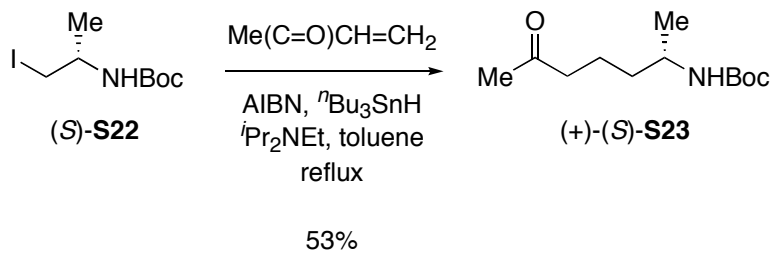

\section{(+)-(S)-(1-Methyl-5-oxo-hexyl)-carbamic acid tert-butyl ester (S23):}

A solution of alkyl iodide $(S)-\mathbf{S 2 2}^{13}(5.10 \mathrm{~g}, 17.7 \mathrm{mmol}, 1$ equiv) in toluene $(95 \mathrm{~mL})$ was treated sequentially with methyl vinyl ketone $(9.40 \mathrm{~mL}, 115 \mathrm{mmol}, 6.50$ equiv) and diisopropylethylamine $(10.0 \mathrm{~mL}, 88.5 \mathrm{mmol}, 3.00$ equiv). The reaction mixture was heated to reflux and a solution of tributyltin hydride $(7.5 \mathrm{~mL}, 28 \mathrm{mmol}, 1.6$ equiv) and AIBN (0.40 g, $2.4 \mathrm{mmol}, 0.15$ equiv) in toluene $(30 \mathrm{~mL})$ was added via cannula. After heating at reflux for $1 \mathrm{~h}$, the reaction mixture was cooled to ambient temperature and triethylamine $(10 \mathrm{~mL})$ was added. Excess tributyltin hydride was quenched by dropwise addition of a solution of iodine in toluene $(0.2 \mathrm{M}, 0.5 \mathrm{~mL})$ until an orange color persisted. The orange solution was stirred for $10 \mathrm{~min}$, then a solution of potassium fluoride $(5.2$ $\mathrm{g})$ in water $(40 \mathrm{~mL})$ was added, and the resulting suspension was stirred for $1.5 \mathrm{~h}$. The reaction mixture was filtered through celite, and the filtrate was partitioned between diethyl ether $(100 \mathrm{~mL})$ and water $(50 \mathrm{~mL})$. The aqueous layer was extracted with diethyl ether $(3 \times 100 \mathrm{~mL})$ and the combined organic layers were washed sequentially with saturated aqueous sodium bicarbonate solution $(20 \mathrm{~mL})$ and brine $(20 \mathrm{~mL})$. The organic phases were dried over anhydrous sodium sulfate, were filtered and were concentrated under reduced pressure. Purification of the yellow residue by flash chromatography (silica gel: diam. 7, ht. $15 \mathrm{~cm}$; eluent: hexanes:EtOAc [1:1]) afforded the ketone $(+)-(S)-\mathbf{S 2 3}$ as a brown solid $\left(2.2 \mathrm{~g}, 53 \%,[\alpha]_{\mathrm{D}}^{22}=+3(c 0.5\right.$, EtOAc $\left.)\right)$.

The corresponding enantiomer, ketone $(-)-(R)-\mathbf{S 2 3}\left(6.9 \mathrm{~g}, 59 \%,[\alpha]_{\mathrm{D}}^{22}=-3(c 0.5\right.$, EtOAc $\left.)\right)$, was prepared using the same procedure and starting with alkyl iodide $(R)-\mathbf{S 2 2}$.

\begin{tabular}{|c|c|}
\hline${ }^{1} \mathrm{H}$ NMR $\left(500 \mathrm{MHz}, \mathrm{CDCl}_{3}, 20^{\circ} \mathrm{C}\right)$ & $\begin{array}{l}4.33 \text { (br-s, } 1 \mathrm{H}, \mathrm{NHBoc}), 3.65\left(\mathrm{br}-\mathrm{s}, 1 \mathrm{H},\left(\mathrm{CH}_{3}\right) \mathrm{CHNH}\right), \\
2.52-2.40\left(\mathrm{~m}, 2 \mathrm{H}, \mathrm{C}(\mathrm{O}) \mathrm{CH}_{2}\right), 2.14\left(\mathrm{~s}, 3 \mathrm{H}, \mathrm{CH}_{3} \mathrm{C}(\mathrm{O}),\right. \\
1.66-1.54\left(\mathrm{~m}, 3 \mathrm{H}, \mathrm{CH}_{2} \mathrm{C}\left(\mathrm{CH}_{3}\right),\left(\mathrm{CH}_{3}\right) \mathrm{CHCH}_{2} \mathrm{CH}_{2}\right), 1.45 \\
\left(\mathrm{~s}, 9 \mathrm{H}, \mathrm{C}\left(\mathrm{CH}_{3}\right)_{3}\right), 1.44-1.38\left(\mathrm{~m}, 1 \mathrm{H}, \mathrm{CH}_{2} \mathrm{C}\left(\mathrm{CH}_{3}\right)\right), 1.12 \\
\left(\mathrm{~d}, 3 \mathrm{H}, J=6.6 \mathrm{~Hz}, \mathrm{CH}\left(\mathrm{CH}_{3}\right)\right) .\end{array}$ \\
\hline
\end{tabular}

${ }^{13} \mathrm{C}$ NMR (125 MHz, $\left.\mathrm{CDCl}_{3}, 20^{\circ} \mathrm{C}\right): \quad$ 209.1, 155.6, 79.2, 46.3, 43.4, 36.7, 30.2, 28.6, 21.4, 20.3 .

FTIR (thin film), $\mathrm{cm}^{-1}$ :

HRMS (EI):

TLC (50\% EtOAc in hexane)
3351 (m, N-H), 2975 (m), 1710 (br-s, C=O), 1523 (m), $1172(\mathrm{~m})$.

calcd for $\mathrm{C}_{12} \mathrm{H}_{24} \mathrm{NO}_{3}[\mathrm{M}+\mathrm{H}]^{+}: 230.1751$, found: 230.1752 .

S22, 0.73 (ninhydrin, UV)

S23, 0.52 (ninhydrin, anis)

\footnotetext{
${ }^{13}$ Prepared from L-alaninol; see: Caputo, R.; Cassano, E.; Longobardo, L.; Palumbo, G. Tetrahedron 1995, $51,12337$.
} 


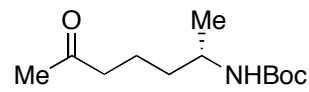

$(+)-(S)-\mathbf{S 2 3}$

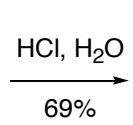

$69 \%$

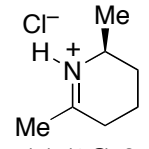

$(-)-(2 S)-6$

\section{(-)-(2S)-2,6-Dimethyl-2,3,4,5-tetrahydro-pyridinium chloride (6):}

The $N$-Boc ketone (+)-(S)-S23 (3.40 g, $14.9 \mathrm{mmol}, 1$ equiv) was dissolved in aqueous hydrochloric acid $(10 \mathrm{M}, 60.0 \mathrm{~mL})$. After stirring for $12 \mathrm{~h}$, the reaction mixture was concentrated under reduced pressure ( 1 Torr) to give a dark brown oil. This residue was triturated from THF $(2 \times$ $10 \mathrm{~mL})$ to provide the iminium chloride $(-)-(2 S)-6$ as a beige solid $\left(1.5 \mathrm{~g}, 69 \%,[\alpha]_{\mathrm{D}}^{22}=-19(c 0.5\right.$, EtOAc)) and vigorously dried. ${ }^{14}$ The optical activity of (-)-6 was measured to be $>99 \%$ ee by chiral HPLC analysis of the corresponding benzylated derivative ${ }^{15}[(S, S)-$ Whelk-O; $3.0 \mathrm{~mL} / \mathrm{min} ; 13 \%$ ${ }^{i} \mathrm{PrOH}$ in hexanes; $t_{\mathrm{R}}$ (major) $=19.53 \mathrm{~min} ; t_{\mathrm{R}}($ minor, not seen $\left.)=17.64 \mathrm{~min}\right]$.

The corresponding enantiomer, iminium chloride $(+)-(2 R)-6\left(0.4 \mathrm{~g}, 81 \%,[\alpha]_{\mathrm{D}}^{22}=+19(c 0.5\right.$, EtOAc)), was prepared using the same procedure and starting with $N$-Boc ketone $(-)-(R)-\mathbf{S 2 3}$. The optical activity of (+)-6 was measured to be $>99 \%$ ee by chiral HPLC analysis of the corresponding benzylated derivative ${ }^{15}\left[(S, S)\right.$-Whelk-O; $3.0 \mathrm{~mL} / \mathrm{min} ; 13 \%{ }^{i} \mathrm{PrOH}$ in hexanes; $t_{\mathrm{R}}$ (major) $=17.64 \mathrm{~min}$; $t_{\mathrm{R}}($ minor, not seen $\left.)=19.53 \mathrm{~min}\right]^{16}$.

${ }^{1} \mathrm{H} \mathrm{NMR}\left(500 \mathrm{MHz}, \mathrm{CDCl}_{3}, 20^{\circ} \mathrm{C}\right)$ :

$15.24\left(\mathrm{~m}, 1 \mathrm{H}, \mathrm{C}=\mathrm{NH}^{+}\right), 3.97$ (br-s, $1 \mathrm{H}$, $\left.\mathrm{NH}^{+} \mathrm{CH}\left(\mathrm{CH}_{3}\right) \mathrm{CH}_{2}\right), 2.64$ (br-s, 5H, $\left(\mathrm{CH}_{3}\right) \mathrm{CNH}^{+}=\mathrm{C}$, $\left.\mathrm{CH}_{3} \mathrm{CCH}_{2}\right), 2.08-2.02\left(\mathrm{~m}, 1 \mathrm{H},\left(\mathrm{CH}_{3}\right) \mathrm{CCH}_{2}\right), 1.97-1.90$ $\left(\mathrm{m}, 1 \mathrm{H},\left(\mathrm{CH}_{3}\right) \mathrm{CCH}_{2}\right), 1.84-1.76(\mathrm{~m}, 1 \mathrm{H}$, $\left.\mathrm{HN}^{+}=\mathrm{CCH}_{2} \mathrm{CH}_{2}\right), 1.61\left(\mathrm{~d}, 3 \mathrm{H}, J=6.8 \mathrm{~Hz}, \mathrm{C}\left(\mathrm{CH}_{3}\right), 1.58-\right.$ $1.55\left(\mathrm{~m}, 1 \mathrm{H}, \mathrm{HN}^{+}=\mathrm{CCH}_{2} \mathrm{CH}_{2}\right)$.

${ }^{13} \mathrm{C}$ NMR $\left(125 \mathrm{MHz}, \mathrm{CDCl}_{3}, 20^{\circ} \mathrm{C}\right)$ :

FTIR (thin film), $\mathrm{cm}^{-1}$ :

HRMS (EI):
$186.6,51.9,31.6,27.5,24.4,20.1,16.0$.

3406 (br-s, N-H), 2936 (m), 2839 (m), 1686 (m), 1457

(w), $1386(\mathrm{w})$.

calcd for $\mathrm{C}_{7} \mathrm{H}_{14} \mathrm{~N}[\mathrm{M}-\mathrm{Cl}]^{+}:$112.1121,

found: 112.1120 .

\footnotetext{
${ }^{14}$ Iminium chloride 6 was highly hygroscopic and required handling under inert atmosphere for optimal results.

${ }^{15}$ Iminium chloride 6 was lithiated and acylated with benzoyl chloride to give the corresponding vinylogous amide.

${ }^{16}$ Additionally, to ensure elution of the major compound after the minor compound, the $(R, R)$-Whelk-O was also used: [ $(R, R)$-Whelk$\mathrm{O} ; 3.0 \mathrm{~mL} / \mathrm{min} ; 13 \%{ }^{i} \mathrm{PrOH}$ in hexanes; $t_{\mathrm{R}}($ major $)=20.34 \mathrm{~min} ; t_{\mathrm{R}}($ minor, not seen $\left.)=19.39 \mathrm{~min}\right]$.
} 


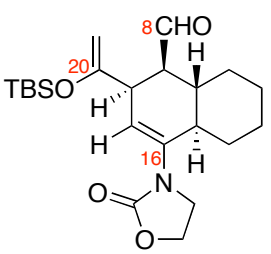

$( \pm)-7$
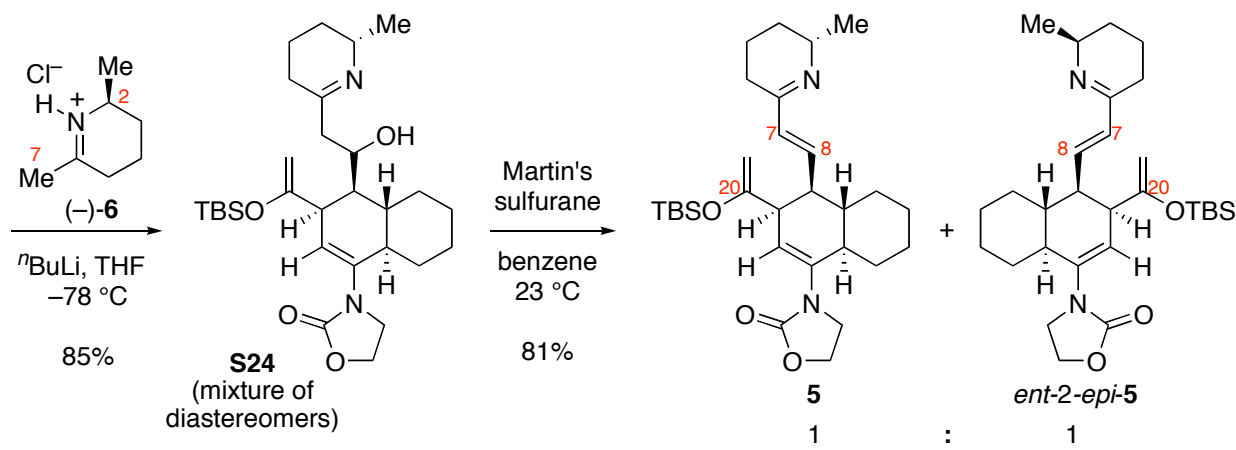

3-\{3-[1-tert-Butyl-dimethyl-silanyloxy)-vinyl]-4-[2-(6-methyl-3,4,5,6-tetrahydro-pyridin-2-yl)-vinyl]3,4,4a,5,6,8,8s-octahydro-naphthalene-1-yl\}-oxazolidin-2-one (5):

To a suspension of iminium chloride (-)-(2S)-6 (101 $\mathrm{mg}, 0.68 \mathrm{mmol}, 2.00$ equiv) in THF at $78{ }^{\circ} \mathrm{C}$ and sealed under $\operatorname{argon}{ }^{17}$ was added a solution of $n$-butyllithium in hexanes $(2.53 \mathrm{M}, 520 \mu \mathrm{L}$, $1.32 \mathrm{mmol}, 3.87$ equiv). The resulting brown solution was maintained at $-78^{\circ} \mathrm{C}$ for $30 \mathrm{~min}$, was warmed to $0{ }^{\circ} \mathrm{C}$ for $10 \mathrm{~min}$, then cooled to $-78^{\circ} \mathrm{C}$. A sample of aldehyde $( \pm)-7(137 \mathrm{mg}, 0.34 \mathrm{mmol}$, 1 equiv) in a round-bottomed flask was azeotropically dried from toluene $(2 \times 4 \mathrm{~mL})$, the flask was evacuated and backfilled with argon three times, charged with THF $(700 \mu \mathrm{L})$, and cooled to $-78{ }^{\circ} \mathrm{C}$. The lithiated enamine solution was transferred cold via cannula to the cold aldehyde solution. After ten minutes excess anion was quenched at $-78{ }^{\circ} \mathrm{C}$ by the addition of saturated aqueous ammonium chloride solution $(2 \mathrm{~mL})$ and the reaction mixture was allowed to warm to room temperature. The reaction mixture was diluted with ethyl acetate $(40 \mathrm{~mL})$ and saturated aqueous ammonium chloride solution $(15 \mathrm{~mL})$ and the layers separated. The aqueous layer was extracted with ethyl acetate $(2 \times$ $40 \mathrm{~mL})$ and the combined organic layers were washed with brine $(15 \mathrm{~mL})$, were dried over anhydrous sodium sulfate, were filtered, and were concentrated under reduced pressure. The resulting yellow oil was purified by flash column chromatography (neutralized silica gel: diam. $2.5 \mathrm{~cm}$, ht. $10 \mathrm{~cm}$; eluent: $\mathrm{CH}_{2} \mathrm{Cl}_{2}$ :acetone: $\mathrm{NEt}_{3}$ [98:1:1] to $\mathrm{CH}_{2} \mathrm{Cl}_{2}$ :acetone: $\mathrm{NEt}_{3}$ [97:2:1] to $\mathrm{CH}_{2} \mathrm{Cl}_{2}:$ acetone: $\mathrm{NEt}_{3}$ [96:3:1]) to provide $\beta$-hydroxyimine $\mathbf{S 2 4}$ (168 $\mathrm{mg}$, 85\%, equal mixture of 4 diastereomers) as a light yellow oil. Additionally, the starting aldehyde $( \pm)-7$ was recovered $(8.1 \mathrm{mg}, 6 \%)$.

A solution of Martin sulfurane (219 mg, $0.326 \mathrm{mmol}, 1.18$ equiv) in benzene $(2 \mathrm{~mL})$ under argon atmosphere, ${ }^{17}$ was transferred via cannula to a solution of $\beta$-hydroxyimine $\mathbf{S 2 4}$ (153 mg, 0.276 mmol, 1 equiv) in benzene $(4 \mathrm{~mL})$ at $23{ }^{\circ} \mathrm{C}$. After $25 \mathrm{~min}$, the reaction mixture was concentrated under reduced pressure and the resulting oil was purified by flash column chromatography (neutralized silica gel: diam. $5 \mathrm{~cm}$, ht. $18 \mathrm{~cm}$; eluent: hexanes:acetone: $\mathrm{NEt}_{3}$ [89:10:1] to acetone:hexanes: $\mathrm{NEt}_{3}$ [84:15:1] to acetone:hexanes: $\mathrm{NEt}_{3}$ [74:25:1] to acetone:hexanes: $\mathrm{NEt}_{3}$ [64:35:1]) to provide the $\alpha, \beta$-unsaturated imines $(119 \mathrm{mg}, 81 \%, \mathbf{5}$ :ent-2-epi-5, 1:1) as a yellow oil.

The corresponding enantiomers of the $\beta$-hydroxyimine $(203 \mathrm{mg}, 70 \%$, equal mixture of 4 diastereomers) and the $\alpha, \beta$-unsaturated imine (167 mg, 85\%, ent-5:2-epi-5, $1: 1)$ were prepared using the same procedure and the imine salt (+)-(2R)-6.

${ }^{1} \mathrm{H}$ NMR (500 MHz, $\mathrm{C}_{6} \mathrm{D}_{6}, 20^{\circ} \mathrm{C}$, equal mixture of two diastereomers, 5:ent-2-epi-5, 1:1): 6.46 (d, $J$ $=4.6 \mathrm{~Hz}, 1 \mathrm{H}, \mathrm{C} 7-\mathrm{H}), 6.43(\mathrm{~d}, J=4.6 \mathrm{~Hz}, 1 \mathrm{H}, \mathrm{C} 7-\mathrm{H})$, 6.13-6.05 (m, 2H, C8-H, C8-H), 5.14-5.11 (m, 2H, C17$\mathrm{H}, \mathrm{C} 17-\mathrm{H}), 4.29$ (br-s, 2H, C21-H, C21-H), 3.62-3.52 (m, 2H, C2-H, C2-H), 3.46-3.41 (m, 4H, OCH $\mathrm{CH}_{2} \mathrm{~N}$, $\left.\mathrm{OCH}_{2} \mathrm{CH}_{2} \mathrm{~N}\right), 2.95-2.89\left(\mathrm{~m}, 2 \mathrm{H}, \mathrm{OCH}_{2} \mathrm{CH}_{2} \mathrm{~N}\right.$,

\footnotetext{
${ }^{17}$ Rigorous inert atmosphere and anhydrous conditions were required for optimal results.
} 
$\left.\mathrm{OCH}_{2} \mathrm{CH}_{2} \mathrm{~N}\right), 2.79-2.75$ (m, 2H, C19-H, C19-H), 2.65$2.60\left(\mathrm{~m}, 2 \mathrm{H}, \mathrm{OCH}_{2} \mathrm{CH}_{2} \mathrm{~N}, \mathrm{OCH}_{2} \mathrm{CH}_{2} \mathrm{~N}\right), 2.40-2.31(\mathrm{~m}$, 4H, C5-H, C5-H, C15-H, C15-H), 2.19-2.07 (m, 6H, C5-H, C5-H, C9-H, C9-H, CH, $\mathrm{CH}_{2}$ ), 2.01-1.94 (m, 2H, C10-H, C10-H), 1.86-1.71 (m, 8H, CH, $\mathrm{CH}_{2}$ ), 1.57-1.49 (m, 8H, C3-H, C3-H, C4-H, C4-H), 1.39 (br-s, 3H, C1-H), 1.38 (br-s, 3H, C1-H), 1.36-1.21 (m, 6H, $\mathrm{CH}_{2}, \mathrm{CH}_{2}$ ), 0.97 (br-s, 18H, $\left.\mathrm{SiC}\left(\mathrm{CH}_{3}\right)_{3}\right), 0.19$ (br-s, 3H, $\left.\mathrm{Si}\left(\mathrm{CH}_{3}\right)_{2}\right), 0.18$ (br-s, 3H, $\left.\mathrm{Si}\left(\mathrm{CH}_{3}\right)_{2}\right), 0.17$ (br-s, 3H, $\left.\mathrm{Si}\left(\mathrm{CH}_{3}\right)_{2}\right), 0.17$ (br-s, 3H, $\left.\mathrm{Si}\left(\mathrm{CH}_{3}\right)_{2}\right)$.

${ }^{13} \mathrm{C}$ NMR (125 MHz, $\mathrm{C}_{6} \mathrm{D}_{6}, 20^{\circ} \mathrm{C}$, equal mixture of two diastereomers, 5:ent-2-epi-5, 1:1): 163.99, 163.98, 159.64, 159.63, 156.01, 155.99, 140.46 (br-s, 2 carbons), 137.99 (C8), 137.95 (C8), 136.26 (C7), 136.19 (C7), 119.44 (C17), 119.25 (C17), 94.39 (C21), 94.34 (C21), 61.80 (br-s, 2 carbons, $\mathrm{OCH}_{2} \mathrm{CH}_{2} \mathrm{~N}$, $\left.\mathrm{OCH}_{2} \mathrm{CH}_{2} \mathrm{~N}\right), 54.53,54.41,48.05$ (C19), 48.04 (C19), $46.82\left(\mathrm{OCH}_{2} \mathrm{CH}_{2} \mathrm{~N}\right), 46.78\left(\mathrm{OCH}_{2} \mathrm{CH}_{2} \mathrm{~N}\right), 46.63(\mathrm{C} 9)$, 46.60 (C9), 43.21 (C15), 43.16 (C15), 40.39 (C10), 40.36 (C10), 31.74 (C11), 31.67 (C11), 30.65 (C14), 30.61 (C14), 30.42, 30.37, 27.39, 27.36, 27.11 (br-s, 2 carbons), 26.31, 26.29, 25.88, 25.85, 24.30, 24.26, 19.66, 19.47, $18.69\left(\mathrm{SiC}\left(\mathbf{C H}_{3}\right)_{3}\right),-4.03\left(\mathrm{Si}\left(\mathbf{C H}_{3}\right)_{2}\right)$, $-4.05\left(\mathrm{Si}\left(\mathrm{CH}_{3}\right)_{2}\right),-4.17\left(\mathrm{Si}\left(\mathrm{CH}_{3}\right)_{2}\right),-4.23\left(\mathrm{Si}\left(\mathbf{C H}_{3}\right)_{2}\right)$.

FTIR (thin film, equal mixture of two diastereomers, 5:ent-2-epi-5, 1:1) $\mathrm{cm}^{-1}: 2929$ (s), $2856(\mathrm{~m})$, 1756 (s), 1615 (m), $1406(\mathrm{~m}), 1259(\mathrm{~m}), 1215(\mathrm{~m}), 839$ (s).

HRMS (ESI, S24):

HRMS (ESI, 5:ent-2-epi-5, 1:1):

TLC Rf (neutralized plates):

$\left(\mathrm{CH}_{2} \mathrm{Cl}_{2}\right.$ :acetone: $\mathrm{NEt}_{3}$ [96:3:1])

(hexanes:acetone: $\mathrm{NEt}_{3}[69: 30: 1]$ ) calcd for $\mathrm{C}_{29} \mathrm{H}_{47} \mathrm{~N}_{2} \mathrm{O}_{4} \mathrm{Si}[\mathrm{M}+\mathrm{H}]^{+}$: 517.3456, found: 517.3464 .

calcd for $\mathrm{C}_{29} \mathrm{H}_{46} \mathrm{~N}_{2} \mathrm{O}_{3} \mathrm{Si}[\mathrm{M}]^{+}$: 499.3351, found: 499.3354.

7, 0.59 (CAM)

S24, 0.21 (CAM)

S24, 0.40 (UV, CAM)

5 and ent-2-epi-5, 0.44 (UV, CAM) 


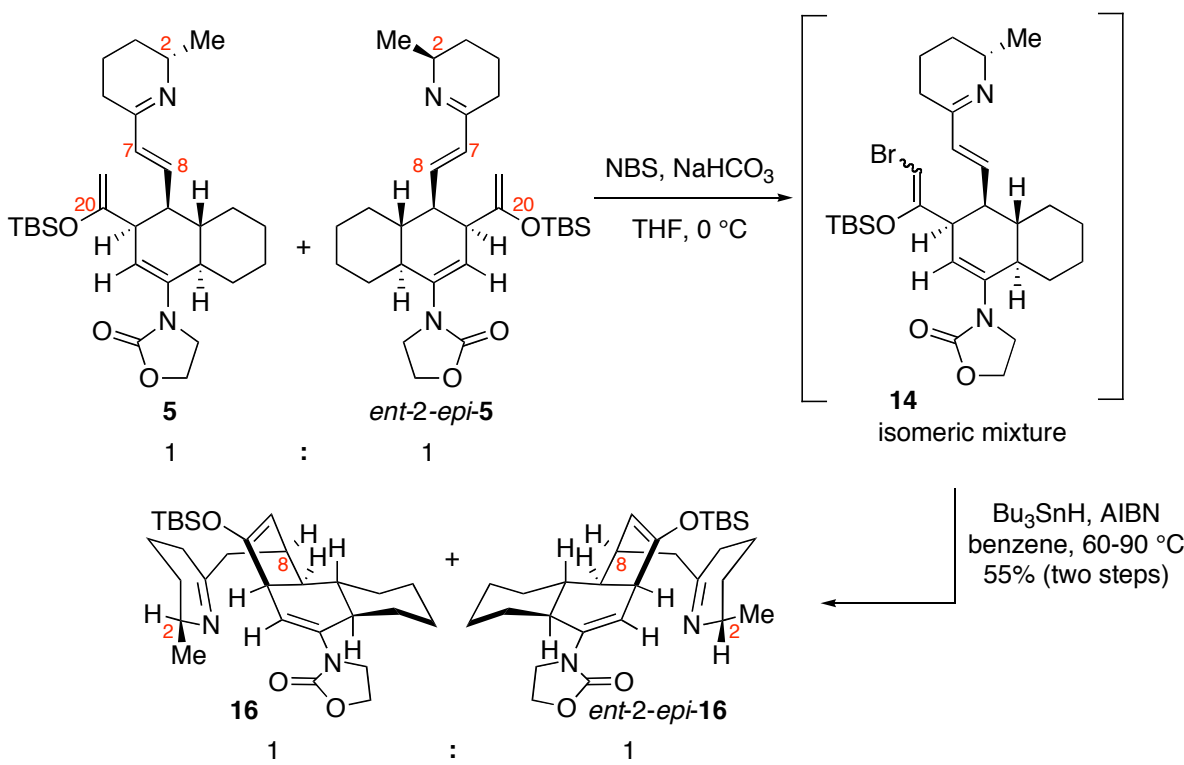

3-[3-(tert-Butyl-dimethyl-silanyloxy)-1-(6-methyl-3,4,5,6-tetrahydro-pyridin-2-ylmethyl)-

3a,5a,6,7,8,9,9a,9b-octahydro-1H-cyclopenta[ $a]$ naphthalen-5-yl]-oxazolidin-2-one (16 and ent-2-epi-16):

A solution of $\alpha, \beta$-unsaturated imine $5(119 \mathrm{mg}, 0.238 \mathrm{mmol}, 1$ equiv, equal mixture of 5 and ent-2-epi-5) in THF (12 mL) was degassed via an argon purge. To this solution was added solid sodium bicarbonate (106 mg, $1.26 \mathrm{mmol}, 5.29$ equiv) under argon. The reaction mixture was cooled to $0{ }^{\circ} \mathrm{C}$, light was excluded, and NBS $(50.5 \mathrm{mg}, 0.284 \mathrm{mmol}, 1.19$ equiv) was added as a solid. The reaction mixture was maintained at $0{ }^{\circ} \mathrm{C}$ for ten minutes, then diluted with hexanes:acetone: $\mathrm{NEt}_{3}$ ([50:50:1], $10 \mathrm{~mL}$ ), was filtered cold through a silica plug (diam. $1 \mathrm{~cm}$, ht. $2.5 \mathrm{~cm}$ ) and the filtrate was concentrated under reduced pressure to produce an orange-brown foam. This residue was dissolved in benzene and filtered to remove excess insoluble succinimide. It was then concentrated and placed under reduced pressure $(\sim 0.5$ Torr $)$ for $1 \mathrm{~h}$. The resulting brominated product was used crude for the cyclization step.

The crude vinyl bromide was dissolved in benzene- $d_{6}{ }^{18}(4.8 \mathrm{~mL})$, was degassed via an argon purge, and was charged with tributyltin hydride $(192 \mu \mathrm{L}, 0.722 \mathrm{mmol}, 3.00$ equiv). A solution of AIBN in benzene- $d_{6}(0.30 \mathrm{M})$ was prepared in a flame-dried flask, degassed via bubbling argon, and a portion $^{19}(200 \mu \mathrm{L}, 0.060 \mathrm{mmol}, 0.25$ equiv) was transferred to the reaction mixture. The reaction solution was placed in a pre-heated $60{ }^{\circ} \mathrm{C}$ oil bath and heated to $90{ }^{\circ} \mathrm{C}$ over $20 \mathrm{~min}$. After $30 \mathrm{~min}$, the reaction mixture was cooled, an additional portion of AIBN was added $(200 \mu \mathrm{L}, 0.060 \mathrm{mmol}, 0.25$ equiv), and the mixture was returned to $90{ }^{\circ} \mathrm{C}$. After a subsequent $20 \mathrm{~min}$, the solution was cooled, additional tributyltin hydride ( $96 \mu \mathrm{L}, 0.36 \mathrm{mmol}, 1.5$ equiv) and AIBN (400 $\mu \mathrm{L}, 0.030 \mathrm{mmol}, 0.50$ equiv) were added, and the reaction was returned to $90^{\circ} \mathrm{C}$. After $20 \mathrm{~min}$, the reaction was cooled, a final portion of $\mathrm{AIBN}$ was added $(400 \mu \mathrm{L}, 0.030 \mathrm{mmol}, 0.50$ equiv), and the mixture was returned to $90{ }^{\circ} \mathrm{C}$. After an additional $20 \mathrm{~min}$ at $90{ }^{\circ} \mathrm{C}$, the reaction appeared complete by direct ${ }^{1} \mathrm{H}$ NMR spectral analysis. The reaction solution was cooled, triethylamine $(1 \mathrm{~mL})$ was added to neutralize adventitious hydrobromic acid, and the solution was concentrated to $400 \mu \mathrm{L}$ under reduced pressure. The resulting brown oil was purified via flash column chromatography (neutralized silica gel: diam. $2.5 \mathrm{~cm}$, ht. $10 \mathrm{~cm}$; eluent: hexanes:acetone: $\mathrm{NEt}_{3}$ [97:2:1] to acetone:hexanes: $\mathrm{NEt}_{3}$ [95:4:1] to acetone:hexanes: $\mathrm{NEt}_{3}$ [92:7:1] to acetone:hexanes: $\mathrm{NEt}_{3}$ [84:15:1]) to provide an equal mixture of two diastereomeric tetracycles $\mathbf{1 6}$ and ent-2-epi-16 as a tan foam (65.7 mg, 55\% (two steps)).

\footnotetext{
${ }^{18}$ Deuterated solvent was used to facilitate evaluation of reaction progress by direct ${ }^{1} \mathrm{H}$ NMR monitoring.

${ }^{19}$ Sequential addition of the reagents was necessary for optimal results.
} 
The corresponding enantiomers, tetracycles ent-16 and 2-epi-16 (90 mg, 54\% (two steps), $(\sim 1: 1))$ were prepared using the same procedure and starting with $\alpha, \beta$-unsaturated imines ent-5:2-epi5, $(\sim 1: 1))$.

${ }^{1} \mathrm{H}$ NMR $\left(500 \mathrm{MHz}, \mathrm{C}_{6} \mathrm{D}_{6}, 20^{\circ} \mathrm{C}\right.$, equal mixture of two diastereomers, 16:ent-2-epi-16, 1:1): 5.665.63 (m, 2H, C17-H, C17-H), 4.83 (app-t, $J=2.9 \mathrm{~Hz}$, 1H, C21-H), 4.70 (app-t, $J=2.9 \mathrm{~Hz}, 1 \mathrm{H}, \mathrm{C} 21-\mathrm{H}$ ), 3.46$3.35\left(\mathrm{~m}, 8 \mathrm{H}, \mathrm{OCH}_{2} \mathrm{CH}_{2} \mathrm{~N}, \mathrm{OCH}_{2} \mathrm{CH}_{2} \mathrm{~N}, \mathrm{C} 2-\mathrm{H}, \mathrm{C} 2-\mathrm{H}\right.$, C19-H, C19-H), 3.11-3.05 (m, 2H, $\mathrm{OCH}_{2} \mathrm{CH}_{2} \mathrm{~N}$, $\mathrm{OCH}_{2} \mathrm{CH}_{2} \mathrm{~N}$ ), 2.99-2.94 (m, $\left.1 \mathrm{H}, \mathrm{C} 8-\mathrm{H}\right), 2.94-2.88$ (m, 1H, C8-H), 2.74-2.69 (m, 2H, $\mathrm{OCH}_{2} \mathrm{CH}_{2} \mathrm{~N}$, $\mathrm{OCH}_{2} \mathrm{CH}_{2} \mathrm{~N}$ ), 2.50-2.44 (m, 2H, C15-H, C15-H), 2.212.15 (m, 4H, C7-H, C7-H, CH, $\mathrm{CH}_{2}$ ), 2.15-2.09 (m, 2H, $\left.\mathrm{CH}_{2}, \mathrm{CH}_{2}\right), 2.09-2.02$ (m, 2H, $\left.\mathrm{CH}_{2}, \mathrm{CH}_{2}\right), 1.79-1.65$ (m, $\left.8 \mathrm{H}, \mathrm{C} 9-\mathrm{H}, \mathrm{C} 9-\mathrm{H}, \mathrm{CH}_{2}, \mathrm{CH}_{2}\right), 1.56-1.43\left(\mathrm{~m}, 4 \mathrm{H}, \mathrm{CH}_{2}\right.$, $\mathrm{CH}_{2}$ ), 1.43-1.26 (m, 4H, C10-H, C10-H, CH, $\left.\mathrm{CH}_{2}\right), 1.36$ $(\mathrm{d}, \mathrm{J}=6.7 \mathrm{~Hz}, 3 \mathrm{H}, \mathrm{C} 1-\mathrm{H}), 1.35(\mathrm{~d}, \mathrm{~J}=6.7 \mathrm{~Hz}, 3 \mathrm{H}, \mathrm{C} 1-$ H), 1.24-1.11 (m, 4H, CH $\mathbf{C H}_{2}$ ), 1.01 (br-s, 18H, $\left.\mathrm{SiC}\left(\mathrm{CH}_{3}\right)_{3}\right), 0.99-0.83\left(\mathrm{~m}, 8 \mathrm{H}, \mathrm{CH}_{2}, \mathrm{CH}_{2}\right), 0.21$ (s, 3H, $\left.\left.\left.\left.\mathrm{SiCH}_{3}\right)_{2}\right), 0.20\left(\mathrm{~s}, 3 \mathrm{H}, \mathrm{SiCH}_{3}\right)_{2}\right), 0.19\left(\mathrm{~s}, 3 \mathrm{H}, \mathrm{SiCH}_{3}\right)_{2}\right)$, $\left.0.18\left(\mathrm{~s}, 3 \mathrm{H}, \mathrm{SiCH}_{3}\right)_{2}\right)$.

${ }^{13} \mathrm{C}$ NMR (125 MHz, $\mathrm{C}_{6} \mathrm{D}_{6}, 20^{\circ} \mathrm{C}$, equal mixture of two diastereomers, 16:ent-2-epi-16, 1:1): 167.31, $167.16,156.35$ (br-s, 2 carbons), 155.48, 155.24, 139.62 (br-s, 2 carbons), 128.93 (br-s, 2 carbons), 118.02, 117.96, 105.25, 104.82, 61.89 (br-s, 2 carbons), 53.97, 53.84, 47.27, 47.24, 46.95, 46.82, 44.87, 44.76, 43.99, 43.95, 43.63 (br-s, 2 carbons), 32.10, 32.01, 30.36 (br-s, 2 carbons), 30.29, 30.24, 30.14, 30.05, 27.38 (br-s, 2 carbons), 27.36, 27.34, 26.34, 26.32, 24.42, 24.38, $19.84,19.67,18.76,18.73,-4.17,-4.18,-4.20,-4.21$.

FTIR (thin film, equal mixture of two diastereomers, 16:ent-2-epi-16, 1:1) $\mathrm{cm}^{-1}$ : 3397 (br-w), 2928 (s), $2856(\mathrm{~m}), 1758(\mathrm{~s}, \mathrm{C}=\mathrm{O}), 1650(\mathrm{~m}), 1408(\mathrm{~m}), 1254$ (m), $1212(\mathrm{w}), 865(\mathrm{~m}), 841(\mathrm{~m}), 782(\mathrm{w})$.

HRMS (ESI): calcd for $\mathrm{C}_{29} \mathrm{H}_{47} \mathrm{~N}_{2} \mathrm{O}_{3} \mathrm{Si}[\mathrm{M}+\mathrm{H}]^{+}:$499.3351, found: 499.3357.

TLC, $R f$ :

( $30 \%$ acetone in hexanes, neutralized plates) 16, 0.44 (UV, CAM) ent-2-epi-16, 0.51 (UV, CAM) 

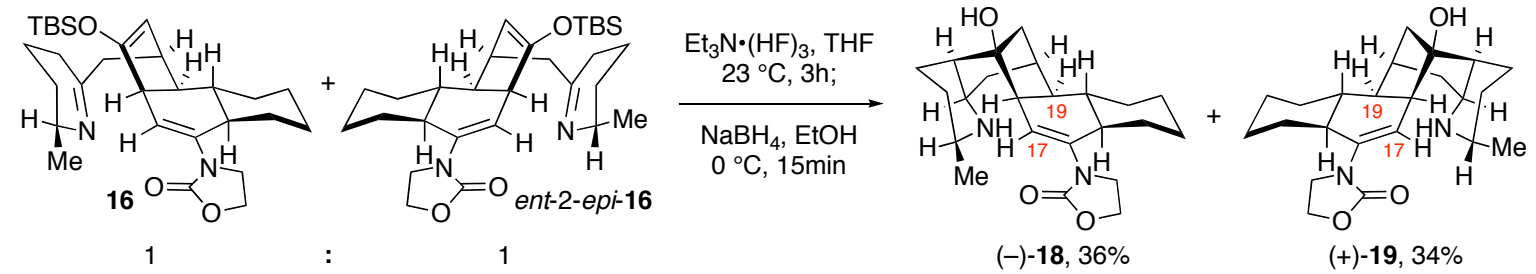

$(+)-19,34 \%$

\section{Pentacyclic amines (-)-18 and (+)-19:}

To a solution of tetracycle $16(65.7 \mathrm{mg}, 0.132 \mathrm{mmol}, 1$ equiv, equal mixture of 16 and ent-2epi-16) in THF $(13 \mathrm{~mL})$ at $23{ }^{\circ} \mathrm{C}$ was added triethylamine trihydrogen fluoride $(107 \mu \mathrm{L}, 0.660 \mathrm{mmol}$, 5.00 equiv). After $3 \mathrm{~h}$, the solution was cooled to $0{ }^{\circ} \mathrm{C}$ and the volatiles were removed under reduced pressure on a manifold and allowed to warm to ambient temperature $(3 \mathrm{~h})$. The crude reaction mixture was dissolved in ethanol $(10 \mathrm{~mL})$ and cooled to $0{ }^{\circ} \mathrm{C}$. A suspension of sodium borohydride (10 $\mathrm{mg}, 0.26 \mathrm{mmol}, 2.0$ equiv) in ethanol $(2 \mathrm{~mL})$ was added dropwise to the cold reaction mixture under an argon atmosphere. The resulting solution was stirred at $0{ }^{\circ} \mathrm{C}$ for ten minutes, then excess hydride was quenched at $0{ }^{\circ} \mathrm{C}$ by the addition of ethanolic hydrochloric acid $(0.5 \mathrm{M}, 1.5 \mathrm{~mL})$ and the solution was vigorously stirred for five minutes. The reaction mixture was neutralized by the addition of triethylamine $(2 \mathrm{~mL})$, was stirred for five minutes, and the volatiles were removed under reduced pressure on a manifold $(1 \mathrm{~h})$. The resulting white solid was dissolved in ethyl acetate (50 $\mathrm{mL})$, saturated aqueous sodium bicarbonate solution $(25 \mathrm{~mL})$ and water $(5 \mathrm{~mL})$. The layers were separated and the aqueous layer was extracted with ethyl acetate $(3 \times 50 \mathrm{~mL})$. The combined organic layers were washed with brine $(10 \mathrm{~mL})$, were dried over anhydrous sodium sulfate, were filtered, and were concentrated under reduced pressure. Purification of the resulting yellow oil via flash column chromatography (neutralized silica gel: diam. $1 \mathrm{~cm}$, ht. $10 \mathrm{~cm}$; eluent: $\mathrm{CH}_{2} \mathrm{Cl}_{2}$ :methanol [95:5] to ammonia saturated $\mathrm{CH}_{2} \mathrm{Cl}_{2}$ :methanol [92:8]) afforded the readily separable pentacyclic amines (-)-18 $\left(18.4 \mathrm{mg}, 36 \%,[\alpha]_{\mathrm{D}}^{22}=-29\left(c 0.44, \mathrm{CH}_{2} \mathrm{Cl}_{2}\right)\right)$ and $(+)-19\left(17.3 \mathrm{mg}, 34 \%,[\alpha]_{\mathrm{D}}^{22}=+65(c 0.43\right.$, $\left.\mathrm{CH}_{2} \mathrm{Cl}_{2}\right)$ ).

The corresponding enantiomeric amines, (+)-18 and (-)-19 (20.0 $\mathrm{mg}$ and $25.0 \mathrm{mg}$, respectively, 66\%), were obtained using the same procedure and starting with a mixture of ent-16 and 2-epi-16 ( 1:1).

${ }^{1} \mathrm{H}$ NMR $\left(500 \mathrm{MHz}, \mathrm{C}_{6} \mathrm{D}_{6}, 20^{\circ} \mathrm{C}\right)$ :

Pentacyclic amine (-)-18: 6.09 (br-s, 1H, C17-H), 3.85$3.80(\mathrm{~m}, 1 \mathrm{H}, \mathrm{C} 19-\mathrm{H}), 3.50$ (app-q, $J=7.4 \mathrm{~Hz}, 1 \mathrm{H}$, $\mathrm{OCH}_{2} \mathrm{CH}_{2} \mathrm{~N}$ ), 3.44 (app-q, $J=6.6 \mathrm{~Hz}, 1 \mathrm{H}, \mathrm{OCH}_{2} \mathrm{CH}_{2} \mathrm{~N}$ ), 3.02-2.91 (m, 2H, $\left.\mathrm{OCH}_{2} \mathrm{CH}_{2} \mathrm{~N}\right), 2.79-2.71(\mathrm{~m}, 2 \mathrm{H}$, $\left.\mathrm{OCH}_{2} \mathrm{CH}_{2} \mathrm{~N}\right), 2.42-2.33(\mathrm{~m}, 1 \mathrm{H}), 2.13(\mathrm{t}, J=9.8 \mathrm{~Hz}$, $1 \mathrm{H}), 2.00-1.94$ (m, 1H), 1.90-1.84 (m, 2H), 1.74 (ddd, $J$ $=14.0,6.2,3.6 \mathrm{~Hz}, 1 \mathrm{H}), 1.71-1.61(\mathrm{~m}, 2 \mathrm{H}), 1.56-1.46$ $(\mathrm{m}, 2 \mathrm{H}), 1.44-1.31(\mathrm{~m}, 3 \mathrm{H}), 1.23-1.09(\mathrm{~m}, 6 \mathrm{H}), 0.98-$ $0.88(\mathrm{~m}, 2 \mathrm{H}) 0.85(\mathrm{~d}, J=6.2 \mathrm{~Hz}, 3 \mathrm{H}, \mathrm{C} 1-\mathrm{H})$.

${ }^{1} \mathrm{H}$ NMR (500 MHz, $\left.\mathrm{C}_{6} \mathrm{D}_{6}, 20^{\circ} \mathrm{C}\right)$ :

Pentacyclic amine (+)-19: 6.00 (br-s, 1H, C17-H), 3.70$3.65(\mathrm{~m}, 1 \mathrm{H}, \mathrm{C} 19-\mathrm{H}), 3.56-3.46\left(\mathrm{~m}, 2 \mathrm{H}, \mathrm{OCH}_{2} \mathrm{CH}_{2} \mathrm{~N}\right)$, $3.26(\mathrm{t}, J=6.1 \mathrm{~Hz}, 1 \mathrm{H}, \mathrm{C} 6-\mathrm{H}), 3.07($ app-q, $J=6.3 \mathrm{~Hz}$, $1 \mathrm{H}, \mathrm{C} 2-\mathrm{H}), 3.04-2.96\left(\mathrm{~m}, 1 \mathrm{H}, \mathrm{OCH}_{2} \mathrm{CH}_{2} \mathrm{~N}\right), 2.77$ (app-q, $\left.J=6.5 \mathrm{~Hz}, 1 \mathrm{H}, \mathrm{OCH}_{2} \mathrm{CH}_{2} \mathrm{~N}\right), 2.07-1.67(\mathrm{~m}, 11 \mathrm{H}), 1.30-$ $1.09(\mathrm{~m}, 6 \mathrm{H}), 1.09-1.02(\mathrm{~m}, 2 \mathrm{H}), 1.00-0.98(\mathrm{~m}, 2 \mathrm{H})$, $0.93(\mathrm{~d}, J=6.5 \mathrm{~Hz}, 3 \mathrm{H}, \mathrm{C} 1-\mathrm{H})$. 
${ }^{13} \mathrm{C}$ NMR $\left(125 \mathrm{MHz}, \mathrm{C}_{6} \mathrm{D}_{6}, 20^{\circ} \mathrm{C}\right)$ :

${ }^{13} \mathrm{C}$ NMR $\left(125 \mathrm{MHz}, \mathrm{C}_{6} \mathrm{D}_{6}, 20^{\circ} \mathrm{C}\right)$ :

FTIR (thin film) $\mathrm{cm}^{-1}$ :

FTIR (thin film) $\mathrm{cm}^{-1}$ :

HRMS (ESI):

HRMS (ESI):

TLC, $R f$ :

(10\% MeOH: $\mathrm{NH}_{3}$ satd $\mathrm{CH}_{2} \mathrm{Cl}_{2}$ )
Pentacyclic amine (-)-18: 157.4 (carbamate $\mathrm{C}=\mathrm{O})$, 137.5 (C16), 128.9 (C17), 80.6 (C20), 61.7 $\left(\mathrm{OCH}_{2} \mathrm{CH}_{2} \mathrm{~N}\right), 56.2,53.8,47.8,47.6,46.3,45.8,45.1$, $42.1,40.6,40.1,38.1,33.8,31.6,29.4,27.1,26.9,24.0$, 23.4 .

Pentacyclic amine (+)-19: 157.5 (carbamate $\mathrm{C}=\mathrm{O}$ ), 138.0 (C16), 128.9 (C17), 81.1 (C20), 61.9 $\left(\mathrm{OCH}_{2} \mathrm{CH}_{2} \mathrm{~N}\right), 48.6,48.0,47.3,46.2,44.0$ (br), 40.9, 40.6, 40.0 (br), 37.6, 33.9, 29.7, 28.2, 27.3, 27.1, 21.1, 20.6 .

Pentacyclic amine (-)-18: 3430 (br-s, OH), 2926 (s), $2855(\mathrm{~m}), 1748(\mathrm{~s}), 1662(\mathrm{~m}), 1481(\mathrm{w}), 1447(\mathrm{w}), 1413$ (m), $1279(\mathrm{w}), 1101(\mathrm{~m}), 735(\mathrm{~m})$.

Pentacyclic amine (+)-19: 3424 (br-s, OH), 2926 (s), $2856(\mathrm{~m}), 1743$ (s), 1666 (w), 1482 (w), 1446 (w), 1416 (m), $1280(\mathrm{w}), 1101(\mathrm{w}), 734(\mathrm{~m})$.

Pentacyclic amine (-)-18: calcd for $\mathrm{C}_{23} \mathrm{H}_{35} \mathrm{~N}_{2} \mathrm{O}_{3}[\mathrm{M}+\mathrm{H}]^{+}$: 387.2642, found: 387.2635 .

Pentacyclic amine (+)-19: calcd for $\mathrm{C}_{23} \mathrm{H}_{35} \mathrm{~N}_{2} \mathrm{O}_{3}[\mathrm{M}+\mathrm{H}]^{+}$: 387.2642, found: 387.2636 .

$(-)-\mathbf{1 8}, 0.63\left(\mathrm{KMnO}_{4}\right)$

$(+)-19,0.26\left(\mathrm{KMnO}_{4}\right)$ 

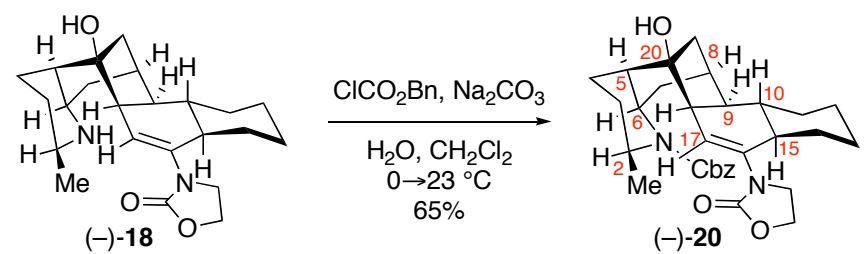

\section{Carbamate (-)-20:}

A solution of sodium carbonate $(21 \mathrm{mg}, 0.19 \mathrm{mmol}, 10$ equiv) in water ( $475 \mu \mathrm{L})$ was added to a solution of amine (-)-18 $\left(7.5 \mathrm{mg}, 0.019 \mathrm{mmol}, 1\right.$ equiv) in dichloromethane $(600 \mu \mathrm{L})$ at $23{ }^{\circ} \mathrm{C}$. The heterogeneous mixture was stirred vigorously and cooled to $0{ }^{\circ} \mathrm{C}$. Benzylchloroformate $(8.2 \mu \mathrm{L}$, $0.057 \mathrm{mmol}, 3.0$ equiv) was added dropwise and the resulting mixture was warmed to room temperature for 15 minutes. Two additional portions of benzylchloroformate $(8.2 \mu \mathrm{L}, 0.057 \mathrm{mmol}$, 3.0 equiv each) were added over 30 minutes, followed by dilution of the reaction mixture with dichloromethane $(10 \mathrm{~mL})$ and saturated aqueous sodium bicarbonate solution $(5 \mathrm{~mL})$. The layers were separated and the aqueous layer was extracted with dichloromethane $(2 \times 10 \mathrm{~mL})$. The combined organic layers were washed with brine $(5 \mathrm{~mL})$, were dried over anhydrous sodium sulfate, were filtered, and were concentrated under reduced pressure. Purification of the resulting yellow oil via flash column chromatography (silica gel: diam. $1 \mathrm{~cm}, \mathrm{ht} .2 .5 \mathrm{~cm}$; eluent: hexanes:acetone [90:10] to hexanes:acetone [80:20] to hexanes:acetone [75:25] to hexanes:acetone [70:30]) provided the carbamate $(-)-20\left([\alpha]_{D}^{22}=-62\left(c 0.6, \mathrm{CH}_{2} \mathrm{Cl}_{2}\right)\right)$ as a clear film $(6.6 \mathrm{mg}, 65 \%)$.

The corresponding enantiomer, carbamate $(+)-20(17.0 \mathrm{mg}, 63 \%)$, was obtained using the same procedure and starting with amine (+)-18.

${ }^{1} \mathrm{H}$ NMR $\left(500 \mathrm{MHz}, \mathrm{C}_{6} \mathrm{D}_{6}, 20^{\circ} \mathrm{C}\right)$ :

$7.32(\mathrm{~d}, J=7.2 \mathrm{~Hz}, 2 \mathrm{H}, \mathrm{ArH}), 7.18(\mathrm{~m}, 2 \mathrm{H}, \mathrm{ArH}), 7.07$ (t, $J=7.4 \mathrm{~Hz}, 1 \mathrm{H}, \mathrm{ArH}), 5.86$ (br-s, 1H, C17-H), 5.27$5.19\left(\mathrm{~m}, 2 \mathrm{H}, \mathrm{PhCH} \mathrm{H}_{2} \mathrm{OC}(\mathrm{O}) \mathrm{N}\right), 4.66-4.57(\mathrm{~m}, 1 \mathrm{H}, \mathrm{C} 6-\mathrm{H})$, 4.39 (br-s, $1 \mathrm{H}, \mathrm{C} 2-\mathrm{H}), 3.55-3.43$ (m, $\left.2 \mathrm{H}, \mathrm{OCH}_{2} \mathrm{CH}_{2} \mathrm{~N}\right)$, 3.01 (app-q, $\left.J=8.3 \mathrm{~Hz}, 1 \mathrm{H}, \mathrm{OCH}_{2} \mathrm{CH}_{2} \mathrm{~N}\right), 2.75$ (td, $J=$ 8.6, $\left.5.9 \mathrm{~Hz}, 1 \mathrm{H}, \mathrm{OCH}_{2} \mathrm{CH}_{2} \mathrm{~N}\right), 2.66-2.61(\mathrm{~m}, 1 \mathrm{H}, \mathrm{C} 19-$ $\mathrm{H}), 2.45-2.37$ (m, 2H, OH, C7-H), 2.09-2.01 (m, 3H, C5-H, C15-H), 1.78-1.65 (m, 3H, C8-H), 1.64 (m, 2H, C21-H), 1.56-1.43 (m, 3H, C4-H, C4-H, C3-H), 1.37$1.28(\mathrm{~m}, 2 \mathrm{H}, \mathrm{C} 3-\mathrm{H}, \mathrm{C} 21-\mathrm{H}), 1.26(\mathrm{dt}, J=13.2,3.2 \mathrm{~Hz}$, 1H), 1.20 (app-t, $J=9.7 \mathrm{~Hz}, 1 \mathrm{H}, \mathrm{C} 9-\mathrm{H}), 1.13(\mathrm{~m}, 1 \mathrm{H})$, 1.12 (d, $J=6.9 \mathrm{~Hz}, 3 \mathrm{H}, \mathrm{C} 1-\mathrm{H}), 1.04-0.95$ (m, 2H, C7$\mathrm{H})$, 0.83-0.74 (m, 2H, C10-H).

${ }^{13} \mathrm{C}$ NMR $\left(125 \mathrm{MHz}, \mathrm{C}_{6} \mathrm{D}_{6}, 20^{\circ} \mathrm{C}\right)$ :

157.3 (carbamate-C=O), $156.0\left(\mathrm{PhCH}_{2} \mathrm{OC}(\mathrm{O}) \mathrm{N}\right), 139.3$ $(\mathrm{C} 16), 138.3\left(\mathrm{HC}=\mathrm{CCH}_{2} \mathrm{OC}(\mathrm{O}) \mathrm{N}\right), 129.1(\mathrm{Ar}-\mathrm{C}), 128.9$ (Ar-C), 128.6 (Ar-C), 127.9 (Ar-C), 123.6 (br, C17), $80.5(\mathrm{C} 20), 67.4\left(\mathrm{PhCH}_{2} \mathrm{OC}(\mathrm{O}) \mathrm{N}\right), 62.0\left(\mathrm{OCH}_{2} \mathrm{CH}_{2} \mathrm{~N}\right)$, 52.9 (C9), 48.1 (C5), $48.0\left(\mathrm{OCH}_{2} \mathbf{C H}_{2} \mathrm{~N}\right), 47.9$ (C6), 46.8 (C2), 45.0 (C10), 40.8 (C15), 39.4 (C19), 34.7 (C21), 34.4 (C4), 33.5 (C8), 33.0, 30.2 (C3), 29.6, 27.1, 27.0, 20.7 (C1), 17.8 (C4). 
FTIR (thin film) $\mathrm{cm}^{-1}$ :

HRMS (ESI):

TLC, $R f$ :

(50\% acetone:hexanes)
3427 (br-s, OH), 2928 (s), 2855 (w), 1733 (s), 1688 (s), 1415 (s), 1316 (s), 1093 (s).

calcd for $\mathrm{C}_{31} \mathrm{H}_{40} \mathrm{NaN}_{2} \mathrm{O}_{5}[\mathrm{M}+\mathrm{Na}]^{+}:$543.2829, found: 543.2808 .

18, $<0.05\left(\mathrm{KMnO}_{4}\right)$

20, $0.31\left(\mathrm{KMnO}_{4}\right)$

NOESY correlations $\left(600 \mathrm{MHz}, \mathrm{C}_{6} \mathrm{D}_{6}, 20^{\circ} \mathrm{C}\right)$ : Additional data: $\mathrm{H} 2-\mathrm{H} 3, \mathrm{H} 3-\mathrm{H} 4, \mathrm{H} 4-\mathrm{H} 5, \mathrm{H} 4-\mathrm{H} 19, \mathrm{H} 3-$ H5, H5-H6, H6-H7a, H6-H21a, H7a-H8, H7b-H9, H9H19, H17-H19, H19-H4a,b. Key correlations are shown in bold.

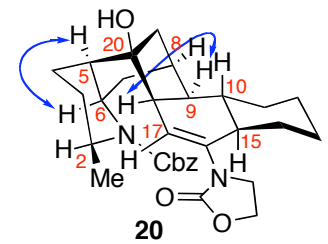




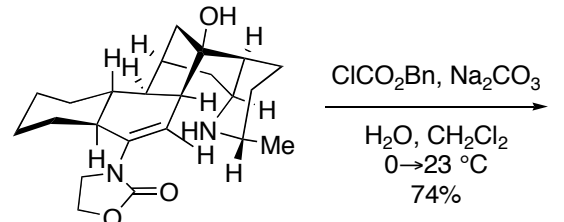

$(+)-19$

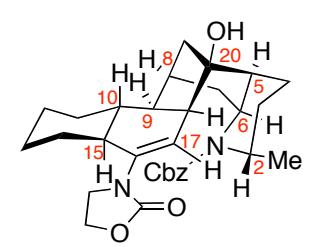

$(+)-2-e p i-20$

\section{Carbamate (+)-2-epi-20:}

A solution of sodium carbonate $(62 \mathrm{mg}, 0.58 \mathrm{mmol}, 10$ equiv) in water $(1 \mathrm{~mL})$ was added to a solution of pentacyclic amine (+)-19 $(22.5 .0 \mathrm{mg}, 0.0580 \mathrm{mmol}, 1$ equiv) in dichloromethane $(1.2 \mathrm{~mL})$ at $23{ }^{\circ} \mathrm{C}$. The heterogeneous mixture was stirred vigorously and cooled to $0{ }^{\circ} \mathrm{C}$. Benzylchloroformate ( $25 \mu \mathrm{L}, 0.18 \mathrm{mmol}, 3.0$ equiv) was added dropwise and the resulting solution was warmed to room temperature for 15 minutes. The reaction mixture was diluted with dichloromethane $(15 \mathrm{~mL})$ and saturated aqueous sodium bicarbonate solution $(10 \mathrm{~mL})$, and water (3 $\mathrm{mL})$. The layers were separated and the aqueous layer was extracted with dichloromethane $(4 \times 15$ $\mathrm{mL})$. The combined organic layers were washed with brine $(20 \mathrm{~mL})$, were dried over anhydrous sodium sulfate, were filtered, and were concentrated under reduced pressure. Purification of the resulting yellow oil via flash column chromatography (silica gel: diam. $1 \mathrm{~cm}$, ht. $7.5 \mathrm{~cm}$; eluent: hexanes:acetone [90:10] to hexanes:acetone [80:20] to hexanes:acetone [75:25] to hexanes:acetone [70:30]) provided the carbamate $(+)-2-e p i-20\left([\alpha]^{22}{ }_{D}=+63\left(c 0.7, \mathrm{CH}_{2} \mathrm{Cl}_{2}\right)\right)$ as a clear film $(22.4 \mathrm{mg}$, $74 \%)$.

The corresponding enantiomer, carbamate (-)-2-epi-20 (18.2 mg, 54\%), was obtained using the same procedure and starting with amine (-)-19.

${ }^{1} \mathrm{H}$ NMR $\left(500 \mathrm{MHz}, \mathrm{C}_{6} \mathrm{D}_{6}, 20^{\circ} \mathrm{C}\right)$ :

${ }^{13} \mathrm{C}$ NMR $\left(125 \mathrm{MHz}, \mathrm{C}_{6} \mathrm{D}_{6}, 20^{\circ} \mathrm{C}\right)$ : $7.28(\mathrm{~d}, J=7.6 \mathrm{~Hz}, 2 \mathrm{H}, \mathrm{ArH}), 7.08$ (app-t, $J=8.1 \mathrm{~Hz}$, ArH), 5.69 (br-s, 1H, C17-H), 5.21 (d, J=12.4 Hz, 1H, $\left.\mathrm{PhCH}_{2} \mathrm{OC}(\mathrm{O}) \mathrm{N}\right), 5.11(\mathrm{~d}, J=12.4 \mathrm{~Hz}, 1 \mathrm{H}$, $\left.\mathrm{PhCH}_{2} \mathrm{OC}(\mathrm{O}) \mathrm{N}\right), 4.13$ (app-q, $J=8.2 \mathrm{~Hz}, 1 \mathrm{H}, \mathrm{C6}-\mathrm{H}$ ), 4.05 (app-q, $J=6.0 \mathrm{~Hz}, 1 \mathrm{H}, \mathrm{C} 2-\mathrm{H}), 3.53-3.43(\mathrm{~m}, 2 \mathrm{H}$, $\mathrm{OCH}_{2} \mathrm{CH}_{2} \mathrm{~N}$ ), 2.96 (app-q, $\left.J=8.5 \mathrm{~Hz}, 1 \mathrm{H}, \mathrm{OCH}_{2} \mathrm{CH}_{2} \mathrm{~N}\right)$, 2.85-2.80 (m, 1H, C19-H), 2.73-2.67 (m, 1H, $\mathrm{OCH}_{2} \mathrm{CH}_{2} \mathrm{~N}$ ), 2.30-2.21 (m, 2H, C15-H, C5-H), 2.011.97 (m, 3H, C8-H, C10-H), 1.87-1.85 (m, 2H, C9-H, C4-H), 1.79 (br-d, $J=12.6 \mathrm{~Hz}, 1 \mathrm{H}, \mathrm{C} 7-\mathrm{H}), 1.68-1.61$ (m, 3H, C3-H), 1.58-1.53 (m, 2H, C4-H, C21-H), 1.38 $(\mathrm{d}, J=11.4 \mathrm{~Hz}, 1 \mathrm{H}, \mathrm{C} 21-\mathrm{H}), 1.27-1.24(\mathrm{~m}, 2 \mathrm{H}), 1.16-$ 1.14 (m, 2H), 1.15-1.13 (m, 3H, C1-H), 1.09 (m, 2H, C3-H), 0.86-0.85 (m, 2H, C7-H).

157.3 (carbamate-C=O), $156.4\left(\mathrm{PhCH}_{2} \mathrm{OC}(\mathrm{O}) \mathrm{N}\right), 139.2$ (C16), $138.3\left(\mathrm{HC}=\mathrm{CCH}_{2} \mathrm{OC}(\mathrm{O}) \mathrm{N}\right), 129.1(\mathrm{Ar}-\mathrm{C}), 128.9$ (Ar-C), 128.7 (Ar-C), 123.3 (br, C17), 81.3 (C20), 67.1 $\left(\mathrm{PhCH}_{2} \mathrm{OC}(\mathrm{O}) \mathrm{N}\right), 62.0\left(\mathrm{OCH}_{2} \mathrm{CH}_{2} \mathrm{~N}\right), 49.2(\mathrm{C} 2), 49.1$ (C9), 48.8 (C6), $47.6\left(\mathrm{OCH}_{2} \mathrm{CH}_{2} \mathrm{~N}\right), 46.9$ (C15), 43.5, 40.9 (C10), 39.1 (C19), 34.2 (C5), 33.6 (C8), 32.4 (C7), 29.8, 29.7, 28.9 (C3), 27.1, 27.0, 20.1, 18.9 (C4). 
FTIR (thin film) $\mathrm{cm}^{-1}$ :

HRMS (ESI):

TLC, $R f$ :

(50\% acetone:hexanes)
3423 (br-s, OH), 2927 (s), 2855 (w), 1734 (s), 1691 (s), 1407 (m), 1297 (m), 1095 (w), 1039 (w).

calcd for $\mathrm{C}_{31} \mathrm{H}_{40} \mathrm{NaN}_{2} \mathrm{O}_{5}[\mathrm{M}+\mathrm{Na}]^{+}:$543.2829, found: 543.2817 .

19, $<0.05\left(\mathrm{KMnO}_{4}\right)$

2-epi-20, $0.31\left(\mathrm{KMnO}_{4}\right)$

NOESY correlations $\left(600 \mathrm{MHz}, \mathrm{C}_{6} \mathrm{D}_{6}, 20^{\circ} \mathrm{C}\right)$ : Additional data: H1-H6, H5-H6, H9-H19, H17-Hb, H17-H19, H19-H4, H15-H9, H4-H2, H4-H6. Key correlations are shown in bold.

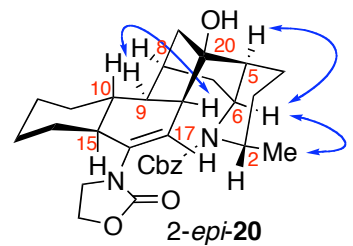



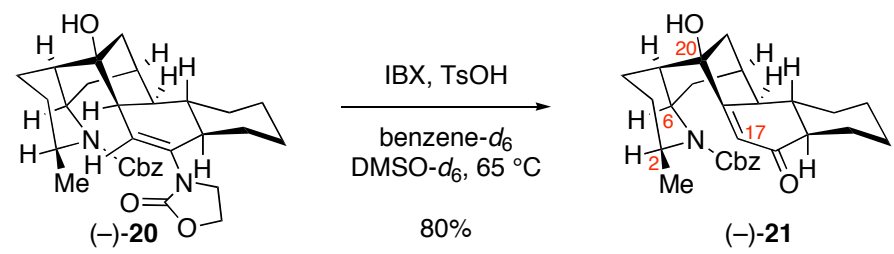

\section{(-)-N-Cbz-Galbulimima alkaloid 13 (21):}

p-Toluenesulfonic acid monohydrate $(12 \mathrm{mg}, 60 \mu \mathrm{mol}, 4.0$ equiv) and IBX (47 mg, 0.16 mmol, 11 equiv) were added to a solution of vinyl oxazolidinone (-)-20 (8.0 mg, $15 \mu \mathrm{mol}, 1$ equiv) in benzene- $d_{6}(300 \mu \mathrm{L})$ and DMSO- $d_{6}{ }^{20}(400 \mu \mathrm{L})$ at $23^{\circ} \mathrm{C}$. The resulting suspension was sonicated $(1.5$ h) until it became homogeneous, then heated to $65{ }^{\circ} \mathrm{C} .{ }^{1} \mathrm{H}$ NMR spectral analysis of the reaction mixture was used to monitor conversion to product. After $10 \mathrm{~h}$, the solution was diluted with ethyl acetate $(10 \mathrm{~mL})$, saturated aqueous sodium bicarbonate solution $(5 \mathrm{~mL})$ and water $(3 \mathrm{~mL})$. The layers were separated and the aqueous layer was extracted with ethyl acetate $(2 \times 10 \mathrm{~mL})$. The combined organic layers were washed with brine $(5 \mathrm{~mL})$, were dried over anhydrous sodium sulfate, were filtered, and were concentrated under reduced pressure. Purification of the resulting yellow residue via flash column chromatography (silica gel: diam. $0.5 \mathrm{~cm}$, ht. $3.5 \mathrm{~cm}$; eluent: hexanes:acetone [85:15] to hexanes:acetone [70:30]) provided enone $(-)-21\left(5.5 \mathrm{mg}, 80 \%,\left([\alpha]^{22}{ }_{\mathrm{D}}=-71(c 0.3\right.\right.$, $\left.\mathrm{CH}_{2} \mathrm{Cl}_{2}\right)$ ).

The corresponding enantiomer, enone (+)-21 (3.5 mg, 67\%) was obtained using the same procedure and starting with vinyl oxazolidinone $(+)-20$.

${ }^{1} \mathrm{H}$ NMR $\left(500 \mathrm{MHz}, \mathrm{C}_{6} \mathrm{D}_{6}, 20^{\circ} \mathrm{C}\right)$ :

${ }^{13} \mathrm{C}$ NMR $\left(125 \mathrm{MHz}, \mathrm{C}_{6} \mathrm{D}_{6}, 20^{\circ} \mathrm{C}\right)$ :

FTIR (thin film) $\mathrm{cm}^{-1}$ :

HRMS (ESI):

TLC, $R f$ :

(50\% acetone:hexanes) $7.32(\mathrm{~d}, J=7.2 \mathrm{~Hz}, 2 \mathrm{H}, \mathrm{ArH}), 7.20-7.15$ (m, 2H, ArH 7.09 (t, $J=7.2 \mathrm{~Hz}, 1 \mathrm{H}, \operatorname{ArH}), 5.99$ (d, $J=2.1 \mathrm{~Hz}, 1 \mathrm{H}$, $\mathrm{C} 17-\mathrm{H}), 5.23$ (m, 2H, PhCH $\left.\left.\mathrm{OC}_{2} \mathrm{O}\right) \mathrm{N}\right), 4.69$ (app-q, $J=$ 8.5 Hz, C6-H), 4.46-4.39 (m, 1H, C2-H), 2.67-2.52 (m, 2H), 1.97-1.90 (m, 1H), 1.75-1.64 (m, 4H), 1.55-1.44 $(\mathrm{m}, 3 \mathrm{H}), 1.38-1.24(\mathrm{~m}, 3 \mathrm{H}), 1.21-1.13(\mathrm{~m}, 2 \mathrm{H}), 1.12-$ $1.04(\mathrm{~m}, 5 \mathrm{H}, \mathrm{C} 1-\mathrm{H}), 1.04-0.85(\mathrm{~m}, 3 \mathrm{H}), 0.84(\mathrm{dd}, J=$ 11.6, $5.2 \mathrm{~Hz}, 1 \mathrm{H}), 0.61$ (app-dq, $J=12.2,3.3 \mathrm{~Hz}, 1 \mathrm{H}$ ) .

199.0 (C16), 172.5 (C19), $155.8\left(\mathrm{PhCH}_{2} \mathrm{OC}(\mathrm{O}) \mathrm{N}\right)$, $138.1\left(\mathrm{HC}=\mathrm{CCH}_{2} \mathrm{OC}(\mathrm{O}) \mathrm{N}\right), 129.1(\mathrm{Ar}-\mathrm{C}), 128.7(\mathrm{Ar}-\mathrm{C})$, 128.5 (Ar-C), 119.3 (C17), 81.1 (C20), 67.5

$\left(\mathrm{PhCH}_{2} \mathrm{OC}(\mathrm{O}) \mathrm{N}\right), 56.7,52.6,47.4,47.3,46.2,45.2$, $36.1,35.7,31.7,30.3,30.2$, 26.9, 26.5, 25.9, 20.4, 19.3.

3428 (br-s, OH), 2924 (s), 2852 (m), 1687 (m), 1666 (s), $1412(\mathrm{w}), 1314(\mathrm{w})$.

calcd for $\mathrm{C}_{28} \mathrm{H}_{37} \mathrm{~N}_{1} \mathrm{O}_{4}[\mathrm{M}+\mathrm{H}]^{+}$: 450.2639, found: 450.2639 .

20, $0.31\left(\mathrm{KMnO}_{4}\right)$

21, 0.57 (UV, CAM)

\footnotetext{
${ }^{20}$ Deuterated solvent was used to facilitate evaluation of reaction progress by direct ${ }^{1} \mathrm{H}$ NMR monitoring.
} 

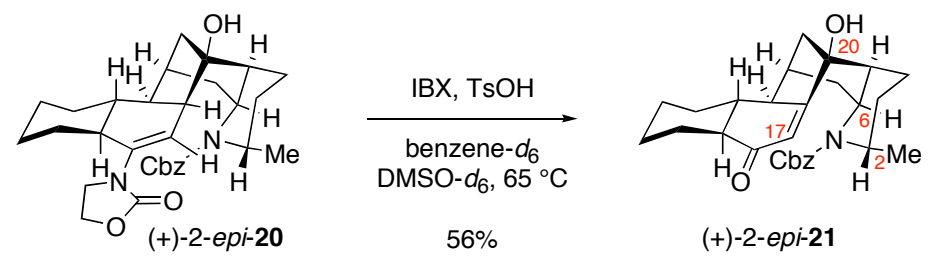

(+)-N-Cbz-2-epi-Galbulimima alkaloid $13(21)$ :

$p$-Toluenesulfonic acid monohydrate $(8.0 \mathrm{mg}, 42 \mu \mathrm{mol}, 4.6$ equiv) and IBX (31 mg, 0.11 mmol, 12 equiv) were added to a solution of vinyl oxazolidinone (+)-2-epi-20 (4.8 mg, $9.2 \mu \mathrm{mol}, 1$ equiv) in benzene- $d_{6}(300 \mu \mathrm{L})$ and DMSO- $d_{6}{ }^{21}(450 \mu \mathrm{L})$ at $23{ }^{\circ} \mathrm{C}$. The suspension was sonicated $(1$ h) until it became homogeneous, then heated to $65^{\circ} \mathrm{C}$. ${ }^{1} \mathrm{H}$ NMR analysis of the reaction mixture was used to monitor conversion to product. After $10 \mathrm{~h}$, the solution was diluted with ethyl acetate $(8 \mathrm{~mL})$, saturated aqueous sodium bicarbonate solution $(8 \mathrm{~mL})$ and water $(3 \mathrm{~mL})$. The layers were separated and the aqueous layer was extracted with ethyl acetate $(3 \times 8 \mathrm{~mL})$. The combined organic layers were washed with brine $(5 \mathrm{~mL})$, were dried over anhydrous sodium sulfate, were filtered, and were concentrated under reduced pressure. Purification of the resulting yellow residue via flash column chromatography (silica gel: diam. $1 \mathrm{~cm}$, ht. $2 \mathrm{~cm}$; eluent: hexanes:acetone [85:15] to hexanes:acetone [80:20]) provided enone (+)-2-epi-21 $\left(2.3 \mathrm{mg}, 56 \%,\left([\alpha]_{\mathrm{D}}^{22}=+70\left(c 0.3, \mathrm{CH}_{2} \mathrm{Cl}_{2}\right)\right)\right.$.

The corresponding enantiomer, enone (-)-2-epi-21 (4.8 $\mathrm{mg}, 59 \%)$ was obtained using the same procedure and starting with vinyl oxazolidinone (-)-2-epi-20.

${ }^{1} \mathrm{H}$ NMR (500 MHz, $\left.\mathrm{C}_{6} \mathrm{D}_{6}, 20^{\circ} \mathrm{C}\right)$ :

${ }^{13} \mathrm{C}$ NMR $\left(125 \mathrm{MHz}, \mathrm{C}_{6} \mathrm{D}_{6}, 20^{\circ} \mathrm{C}\right)$ :

FTIR (thin film) $\mathrm{cm}^{-1}$ :

HRMS (ESI):

TLC, $R f$ :

(50\% acetone:hexanes)
7.30-7.27 (m, 2H, ArH), 7.15-7.12 (m, 2H, ArH), 7.09-

7.05 (m, 1H, ArH), 5.99-5.96 (m, 1H, C17-H), 5.18 (d, J $\left.=12.4 \mathrm{~Hz}, \mathrm{PhCH}_{2} \mathrm{OC}(\mathrm{O}) \mathrm{N}\right), 5.14(\mathrm{~d}, J=12.4 \mathrm{~Hz}$, $\left.\mathrm{PhCH}_{2} \mathrm{OC}(\mathrm{O}) \mathrm{N}\right), 4.50$ (app-q, $\left.J=9.2 \mathrm{~Hz}, 1 \mathrm{H}, \mathrm{C} 6-\mathrm{H}\right)$, 3.71-3.64 (m, 1H, C2-H), 2.64-2.59 (m, 1H), 2.30 (dt, $J$ $=13.8,8.0 \mathrm{~Hz}, 1 \mathrm{H}), 2.23-2.16(\mathrm{~m}, 1 \mathrm{H}), 1.77-1.65(\mathrm{~m}$, $3 \mathrm{H}), 1.64-1.43(\mathrm{~m}, 6 \mathrm{H}), 1.36-1.27(\mathrm{~m}, 2 \mathrm{H}), 1.22(\mathrm{~d}, J=$ $6.5 \mathrm{~Hz}, 3 \mathrm{H}, \mathrm{C} 1-\mathrm{H}), 1.18-1.04(\mathrm{~m}, 4 \mathrm{H}), 1.04-0.96$ (m, $1 \mathrm{H}), 0.96-0.86(\mathrm{~m}, 2 \mathrm{H}), 0.74-0,65(\mathrm{~m}, 1 \mathrm{H})$.

199.0 (C16), 173.3 (C19), $156.6\left(\mathrm{PhCH}_{2} \mathrm{OC}(\mathrm{O}) \mathrm{N}\right)$, $138.1\left(\mathrm{HC}=\mathrm{CCH}_{2} \mathrm{OC}(\mathrm{O}) \mathrm{N}\right), 129.1(\mathrm{Ar}-\mathrm{C}), 128.7(\mathrm{Ar}-\mathrm{C})$, 128.5 (Ar-C), 119.6 (C17), 82.0 (C20), 67.3 $\left(\mathrm{OCH}_{2} \mathrm{CH}_{2} \mathrm{~N}\right), 54.4,52.4,49.1,48.5,47.4,43.1,39.3$, $35.6,31.8,30.6,29.9,27.0,26.5,26.0,21.1,21.0$.

3423 (br-s, OH), 2929 (m), 2857 (w), 1687 (m), 1662 (s), $1300(\mathrm{~m}), 1154(\mathrm{w})$.

calcd for $\mathrm{C}_{28} \mathrm{H}_{37} \mathrm{~N}_{1} \mathrm{O}_{4}[\mathrm{M}+\mathrm{H}]^{+}$: 450.2639, found: 450.2644 .

2-epi-20, $0.31\left(\mathrm{KMnO}_{4}\right)$

2-epi-21, 0.57 (UV, CAM)

\footnotetext{
${ }^{21}$ Deuterated solvent was used to facilitate evaluation of reaction progress by direct ${ }^{1} \mathrm{H}$ NMR monitoring.
} 


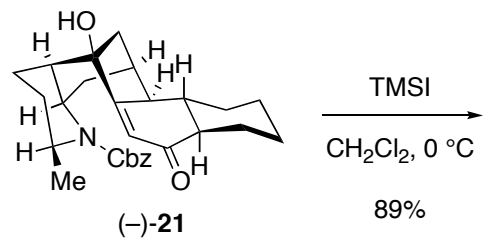

$(-)-21$

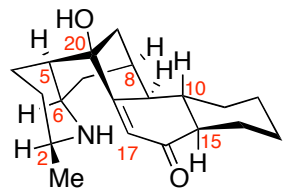

(-)-galbulimima alkaloid $13(2)$

\section{(-)-Galbulimima alkaloid $13(2):^{22}$}

$N$-Cbz enone (-)-21 (2.7 mg, $6.0 \mu \mathrm{mol}, 1$ equiv) was azeotropically dried from toluene $(3 \times 1$ $\mathrm{mL})$, was dissolved in dichloromethane $(1.0 \mathrm{~mL})$, and was cooled to $0{ }^{\circ} \mathrm{C}$. Trimethylsilyliodide (TMSI, $1.2 \mu \mathrm{L}, 9.0 \mu \mathrm{mol}, 1.5$ equiv) was added to the cooled solution, and the resulting yellow solution was stirred at $0{ }^{\circ} \mathrm{C}$. Additional portions of TMSI were added at 20 minute intervals until complete consumption of $\mathbf{2 1}$ was observed by TLC analysis $(70 \mathrm{~min})$. The reaction mixture on completion was a cloudy yellow solution, with a brown residue. Excess silylated products were quenched at $0{ }^{\circ} \mathrm{C}$ by the addition of aqueous hydrochloric acid solution $(1 \mathrm{~N}, 1.5 \mathrm{~mL})$ and the mixture was allowed to warm to ambient temperature. The reaction mixture was diluted with hexanes (10 $\mathrm{mL})$ and aqueous hydrochloric acid solution $(1 \mathrm{~N}, 2 \mathrm{~mL})$ and the layers were separated. The organic layer was extracted with aqueous hydrochloric acid solution $(1 \mathrm{~N}, 4 \mathrm{~mL})$. The combined acidic aqueous layers were washed sequentially with hexanes $(2 \times 10 \mathrm{~mL})$, dichloromethane $(10 \mathrm{~mL})$, and hexanes $(10 \mathrm{~mL})$. The aqueous layer was then basified to $\mathrm{pH} 13$ with aqueous sodium hydroxide solution $(1 \mathrm{~N}, 10 \mathrm{~mL})$. The resulting solution was stirred at ambient temperature for $1 \mathrm{~h}$. The aqueous solution was extracted with dichloromethane $(3 \times 20 \mathrm{~mL})$ and the combined organic layers were washed sequentially with water $(18 \mathrm{~mL})$ and brine $(20 \mathrm{~mL})$, were dried over anhydrous sodium sulfate, were filtered, and were concentrated under reduced pressure to provide (-)-galbulimima alkaloid $13\left(2,[\alpha]_{D}^{22}=-34\left(c 0.045, \mathrm{CH}_{2} \mathrm{Cl}_{2}\right)^{23}\right)$ as a white film $(1.7 \mathrm{mg}, 89 \%)$.

The corresponding enantiomer, (+)-galbulimima alkaloid $13\left(2,1.4 \mathrm{mg}, 58 \%,[\alpha]^{22}=+34(c\right.$ $\left.0.090, \mathrm{CH}_{2} \mathrm{Cl}_{2}\right)^{23}$ ), was obtained.using the same procedure and starting with $\mathrm{N}-\mathrm{Cbz}$ amine ent-21.

${ }^{1} \mathrm{H}$ NMR (500 MHz, $\left.\mathrm{C}_{6} \mathrm{D}_{6}, 20^{\circ} \mathrm{C}\right)$ :

6.07 (d, $J=2.2 \mathrm{~Hz}, 1 \mathrm{H}, \mathrm{C} 17-\mathrm{H}), 3.28(\mathrm{dt}, J=11.4,2.3$

Hz, 1H, C9-H), 2.88 (app-t, $J=5.1 \mathrm{~Hz}, 1 \mathrm{H}, \mathrm{C6}-\mathrm{H}$ ),

2.72-2.66 (m, 1H), 2.60-2.55 (m, 1H), 2.15 (app-qd, $J=$

6.1, $2.3 \mathrm{~Hz}, 1 \mathrm{H}, \mathrm{C} 2-\mathrm{H}), 1.98$ (m, 1H, OH), 1.91 (app-t, $J$

$=4.4 \mathrm{~Hz}, 1 \mathrm{H}, \mathrm{C} 5-\mathrm{H}), 1.84-1.81(\mathrm{~m}, 1 \mathrm{H}, \mathrm{C} 8-\mathrm{H}), 1.78$

(dd, $J=11.2,3.6 \mathrm{~Hz}, 1 \mathrm{H}, \mathrm{C} 15-\mathrm{H}), 1.75-1.69$ (m, 1H),

$1.67-1.60(\mathrm{~m}, 1 \mathrm{H}), 1.55$ (app-dq, $J=13.9,2.9 \mathrm{~Hz}, 1 \mathrm{H}$,

C7-Ha), 1.53-1.47 (m, 1H), 1.40 (ddd, $J=10.8,5.6,2.1$

$\mathrm{Hz}, 1 \mathrm{H}, \mathrm{C} 21-\mathrm{Ha}), 1.26$ (dd, $J=5.6,2.4 \mathrm{~Hz}, 1 \mathrm{H}, \mathrm{C} 21-$

$\mathrm{Hb}), 1.23-0.90$ (m, 8H, C10-H, C7-Hb), 0.77 (m, 1H),

$0.75(\mathrm{~d}, J=6.1 \mathrm{~Hz}, 3 \mathrm{H}, \mathrm{C} 1-\mathrm{H})$.

${ }^{13} \mathrm{C}$ NMR (125 MHz, $\left.\mathrm{C}_{6} \mathrm{D}_{6}, 20^{\circ} \mathrm{C}\right)$ :

199.4 (C16), 178.9 (C19), 119.2 (C17), 79.7 (C20), 55.4 (C6), 53.3 (C15), 53.2 (C2), 51.2 (C9), 48.2 (C21), 47.6

\footnotetext{
22 a) For the prior synthesis of ( \pm )-2, please see: Mander, L. N.; McLachlan, M. M. J. Am. Chem. Soc. 2003, 125, 2400. The Supporting Information of this same report contains copies of the NMR spectra of the natural (-)-2 along with synthetic ( \pm )-2. b) For isolation and optical rotation data, see: Ritchie, E.; Taylor, W. C. In The Alkaloids; Manske, R. H. F., Ed.; Academic Press: New York, 1967; Vol. 9, Chapter 14 and references therein.

${ }^{23}$ Literature value: $[\alpha]=-84\left(\mathrm{CHCl}_{3}\right)$; see reference $22 \mathrm{~b}$. We have also measured the rotation of (-)-2 in chloroform (2 sources): a) chloroform passed through basic alumina (Grade I) and dried over $4 \AA$-MS, $[\alpha]^{22}=-51\left(c 0.06, \mathrm{CHCl}_{3}\right)$, and b) chloroform passed through basic alumina (Grade I) and distilled from $\mathrm{P}_{2} \mathrm{O}_{5},[\alpha]_{\mathrm{D}}^{22}=-64\left(c 0.06, \mathrm{CHCl}_{3}\right)$. Additionally, we have measured the rotation of (+)-2 in chloroform: a) chloroform passed through basic alumina (Grade I) and dried over $4 \AA-\mathrm{MS},[\alpha]^{22}{ }_{\mathrm{D}}=+51\left(c 0.07, \mathrm{CHCl}_{3}\right)$, and b) chloroform passed through basic alumina (Grade I) and distilled from $\mathrm{P}_{2} \mathrm{O}_{5},[\alpha]_{\mathrm{D}}^{22}=+66\left(c 0.07, \mathrm{CHCl}_{3}\right)$.
} 
(C10), 46.6 (C5), 41.0 (C7), 33.1 (C8), 31.9, 30.6, 27.3

(C3 or C4), 26.7, 26.1, 25.0 (C3 or C4), 23.6 (C1).

FTIR (thin film) $\mathrm{cm}^{-1}$ :

3403 (br-s, OH), 2921 (s), 2851 (m), 1708 (w), 1646 (s), 1447 (m), 1261 (m). (Literature values: 3406, 2929, $2854,1705,1646,1446) .^{22}$

HRMS (ESI):

calcd for $\mathrm{C}_{20} \mathrm{H}_{30} \mathrm{~N}_{1} \mathrm{O}_{2}[\mathrm{M}+\mathrm{H}]^{+}:$316.2271,

found: 316.2280 .

NOESY correlations $\left(600 \mathrm{MHz}, \mathrm{C}_{6} \mathrm{D}_{6}, 20^{\circ} \mathrm{C}\right)$ : Additional data: $\mathrm{H} 1-\mathrm{H} 2$, H2-H6, H5-H6, H5-H21b,

H6-H7a, H6-H21b, H7a-H8, H7a-H6, H8-H21a, H9-

H15. Key correlations are shown in bold.

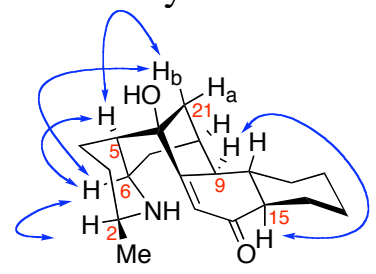

(-)-galbulimima alkaloid $13(2)$

Comparison of our assignments for (-)-galbulimima alkaloid 13 (2) with prior assignments for ( \pm )-2:

\begin{tabular}{|c|c|c|c|c|}
\hline Assignment & $\begin{array}{c}\text { Mander's report }^{22} \\
( \pm)-\mathrm{GB}-13(\mathbf{2}) \\
\left({ }^{1} \mathrm{H}, 300 \mathrm{MHz}, \mathrm{CDCl}_{3}\right)\end{array}$ & $\begin{array}{c}\text { This report } \\
(-)-\mathrm{GB} 13(2) \\
\left({ }^{1} \mathrm{H}, 500 \mathrm{MHz}, \mathrm{CDCl}_{3}\right)\end{array}$ & $\begin{array}{c}\text { This report } \\
(-)-G B 13(2) \\
\left({ }^{1} \mathrm{H}, 500 \mathrm{MHz}, \mathrm{C}_{6} \mathrm{D}_{6}\right)\end{array}$ & $\begin{array}{c}\text { This report } \\
(-) \text {-GB 13 (2) } \\
{ }^{13} \mathrm{C}\left(125 \mathrm{MHz}, \mathrm{C}_{6} \mathrm{D}_{6}\right)\end{array}$ \\
\hline $\mathrm{C} 1$ & $0.89,(\mathrm{~d}, J=6.2 \mathrm{~Hz})$ & $0.89,(\mathrm{~d}, J=6.1 \mathrm{~Hz})$ & $0.75,(\mathrm{~d}, J=6.1 \mathrm{~Hz})$ & 23.6 \\
\hline $\mathrm{C} 2$ & & & $2.15,($ app-qd, $J=6.1,2.3 \mathrm{~Hz})$ & 53.2 \\
\hline $\mathrm{C} 3, \mathrm{C} 4$ & & & $2.72-2.66(\mathrm{~m}) ; 2.60-2.55(\mathrm{~m})$ & $27.3,25.0$ \\
\hline $\mathrm{C} 5$ & & & $1.91($ app-t, $J=4.4 \mathrm{~Hz})$ & 46.6 \\
\hline C6 & $3.34,(\mathrm{t}, J=5.1 \mathrm{~Hz})$ & $3.34,(\mathrm{t}, J=5.1 \mathrm{~Hz})$ & $2.88,($ app-t, $J=5.1 \mathrm{~Hz})$ & 55.4 \\
\hline $\mathrm{C} 7$ & & & $\begin{array}{c}1.55 \text { (app-dq, } J=13.9,2.9) ; \\
1.02(\mathrm{~m})\end{array}$ & 41.0 \\
\hline $\mathrm{C} 8$ & & & $1.84-1.81(\mathrm{~m})$ & 33.1 \\
\hline C9 & $3.47,(\mathrm{dt}, J=11.3,2.2 \mathrm{~Hz})$ & $3.47,(\mathrm{dt}, J=11.2,2.1 \mathrm{~Hz})$ & $3.28,(\mathrm{dt}, J=11.4,2.3 \mathrm{~Hz})$ & 51.2 \\
\hline $\mathrm{C} 10$ & & & $\sim 1.13, \mathrm{~m}$ & 47.6 \\
\hline $\mathrm{C} 11-\mathrm{C} 14$ & & & & $31.9,30.6,26.7,26.1$ \\
\hline $\mathrm{C} 15$ & & & $1.78, \mathrm{dd}, J=11.2,3.6 \mathrm{~Hz}$ & 53.3 \\
\hline $\mathrm{C} 16$ & - & - & - & 199.4 \\
\hline $\mathrm{C} 17$ & $5.92,(\mathrm{~d}, J=2.0 \mathrm{~Hz})$ & $5.93(\mathrm{~d}, J=2.1 \mathrm{~Hz})$ & $6.07,(\mathrm{~d}, J=2.2 \mathrm{~Hz})$ & 119.2 \\
\hline C19 & - & - & - & 178.9 \\
\hline $\mathrm{C} 20$ & - & - & - & 79.7 \\
\hline $\mathrm{C} 21$ & & & $\begin{array}{c}1.40(\mathrm{ddd}, J=10.8,5.6,2.1 \\
\mathrm{Hz}) ; 1.26(\mathrm{dd}, J=5.6,2.4 \mathrm{~Hz})\end{array}$ & 48.2 \\
\hline
\end{tabular}




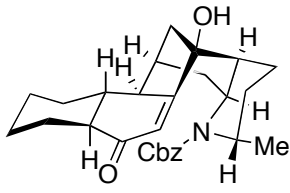

$(+)-2-e p i-21$

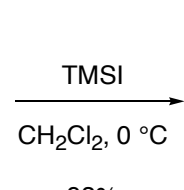

$93 \%$

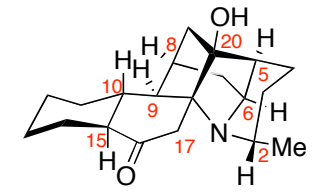

(+)-2-epi-16-oxohimgaline (2-epi-16-oxo-3)

\section{(+)-2-epi-16-Oxohimgaline (2-epi-16-0xo-3):}

$N$-Cbz enone (+)-2-epi-21 (2.1 mg, $4.7 \mu \mathrm{mol}, 1$ equiv) was azeotropically dried from toluene

$(3 \times 1 \mathrm{~mL})$, was dissolved in dichloromethane $(800 \mu \mathrm{L})$, and was cooled to $0{ }^{\circ} \mathrm{C}$. Trimethylsilyliodide (TMSI, $1.5 \mu \mathrm{L}, 11 \mu \mathrm{mol}, 2.2$ equiv) was added to the cooled solution, and the resulting yellow solution was stirred at $0{ }^{\circ} \mathrm{C}$. Additional portions of TMSI were added at 20 minute intervals until complete consumption of $\mathbf{2 1}$ was observed by TLC analysis $(50 \mathrm{~min})$. The reaction mixture on completion was a cloudy yellow solution, with a brown residue. Excess silylated products were quenched at $0{ }^{\circ} \mathrm{C}$ by the addition of aqueous hydrochloric acid solution $(1 \mathrm{~N}, 1.5 \mathrm{~mL})$ and the reaction mixture was allowed to warm to ambient temperature. The reaction mixture was diluted with hexanes $(10 \mathrm{~mL})$ and aqueous hydrochloric acid solution $(1 \mathrm{~N}, 3 \mathrm{~mL})$ and the layers were separated. The organic layer was extracted with aqueous hydrochloric acid solution ( $1 \mathrm{~N}, 4 \mathrm{~mL})$. The combined acidic aqueous layers were washed sequentially with hexanes $(2 \times 10 \mathrm{~mL})$, dichloromethane $(10 \mathrm{~mL})$, and hexanes $(10 \mathrm{~mL})$. The aqueous layer was then basified to $\mathrm{pH} 13$ with aqueous sodium hydroxide solution $(1 \mathrm{~N}, 11 \mathrm{~mL})$. The resulting solution was stirred at ambient temperature for $1 \mathrm{~h}$. The aqueous solution was extracted with dichloromethane $(3 \times 20 \mathrm{~mL})$ and the combined organic layers were washed sequentially with water $(18 \mathrm{~mL})$ and brine $(10 \mathrm{~mL})$, were dried over anhydrous sodium sulfate, were filtered, and were concentrated under reduced pressure to provide (+)-2-epi-oxohimgaline $\left((+)\right.$-2-epi-16-oxo-3, $\left.[\alpha]^{22}{ }_{\mathrm{D}}=+24\left(c 0.07, \mathrm{CH}_{2} \mathrm{Cl}_{2}\right)\right)$ as a white film (1.4 mg, 93\%).

The corresponding enantiomer, (-)-2-epi-oxohimgaline ((-)-2-epi-16-oxo-3, $2.8 \mathrm{mg}, 82 \%$, $\left.[\alpha]_{\mathrm{D}}^{22}=-24\left(c 0.085, \mathrm{CH}_{2} \mathrm{Cl}_{2}\right)\right)$, was obtained using the same procedure and starting with $\mathrm{N}-\mathrm{Cbz}$ enone (-)-2-epi-21.

${ }^{1} \mathrm{H}$ NMR $\left(500 \mathrm{MHz}, \mathrm{C}_{6} \mathrm{D}_{6}, 20^{\circ} \mathrm{C}\right)$ :

${ }^{13} \mathrm{C}$ NMR $\left(125 \mathrm{MHz}, \mathrm{C}_{6} \mathrm{D}_{6}, 20^{\circ} \mathrm{C}\right)$ :

FTIR (thin film) $\mathrm{cm}^{-1}$ :
3.08-3.02 (m, 2H, C2-H, C6-H), 3.02 (d, $J=13.5 \mathrm{~Hz}$, $1 \mathrm{H}, \mathrm{C} 17-\mathrm{Ha}), 2.55$ (br-d, $J=12.9 \mathrm{~Hz}, 1 \mathrm{H}, \mathrm{C} 17-\mathrm{Hb})$, 1.99-1.91 (m, 2H, C9-H, OH), 1.91-1.88 (m, 1H, C8-H), 1.87-1.81 (m, 1H), 1.80-1.72 (m, 2H, C15-H, C21-Ha), 1.68-1.48 (m, 7H, C5-H, C21-Hb, C7-Ha), 1.43-1.30 (m, 3H), 1.26 (br-d, $J=12.7 \mathrm{~Hz}, 1 \mathrm{H}, \mathrm{C} 7-\mathrm{Hb}), 1.14$ (qd, $J=11.4,3.4 \mathrm{~Hz}, 1 \mathrm{H}, \mathrm{C} 10-\mathrm{H}), 1.06(\mathrm{~d}, J=7.0 \mathrm{~Hz}, 3 \mathrm{H}$, $\mathrm{C} 1-\mathrm{H}), 1.02-0.89(\mathrm{~m}, 3 \mathrm{H}), 0.79(\mathrm{dd}, J=13.6,6.5 \mathrm{~Hz}$, $1 \mathrm{H}), 0.73-0.63(\mathrm{~m}, 1 \mathrm{H})$.

212.3 (C16), 87.4 (C20), 75.7 (C19), 60.5 (C9), 57.3 (C6), 55.0 (C5), 52.8 (C15), 50.6 (C2), 47.9 (C21), 44.4 (C10), 40.9 (C17), 37.1 (C7), 36.2 (C8), 32.5, 30.3, 26.4, 25.7, 24.1, 22.4, 20.7 (C1).

3302 (br-m, OH), 2929 (s), 2853 (w), 1706 (m, C=O), $1314(w), 1300(w), 1182(w)$. 
HRMS (ESI):

calcd for $\mathrm{C}_{20} \mathrm{H}_{30} \mathrm{~N}_{1} \mathrm{O}_{2}[\mathrm{M}+\mathrm{H}]^{+}: 316.2271$, found: 316.2270 .

NOESY correlations (600 MHz, $\left.\mathrm{C}_{6} \mathrm{D}_{6}, 20^{\circ} \mathrm{C}\right)$ : H6-H7b, H8-H7a, H8-H7b, H9-H15, H5-H21b, H5H7b, H10-H21a. Key correlations are shown in bold.

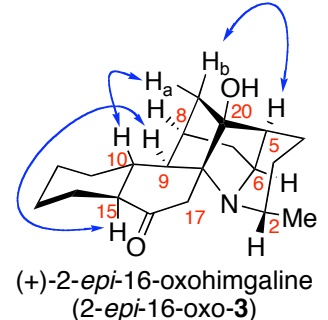




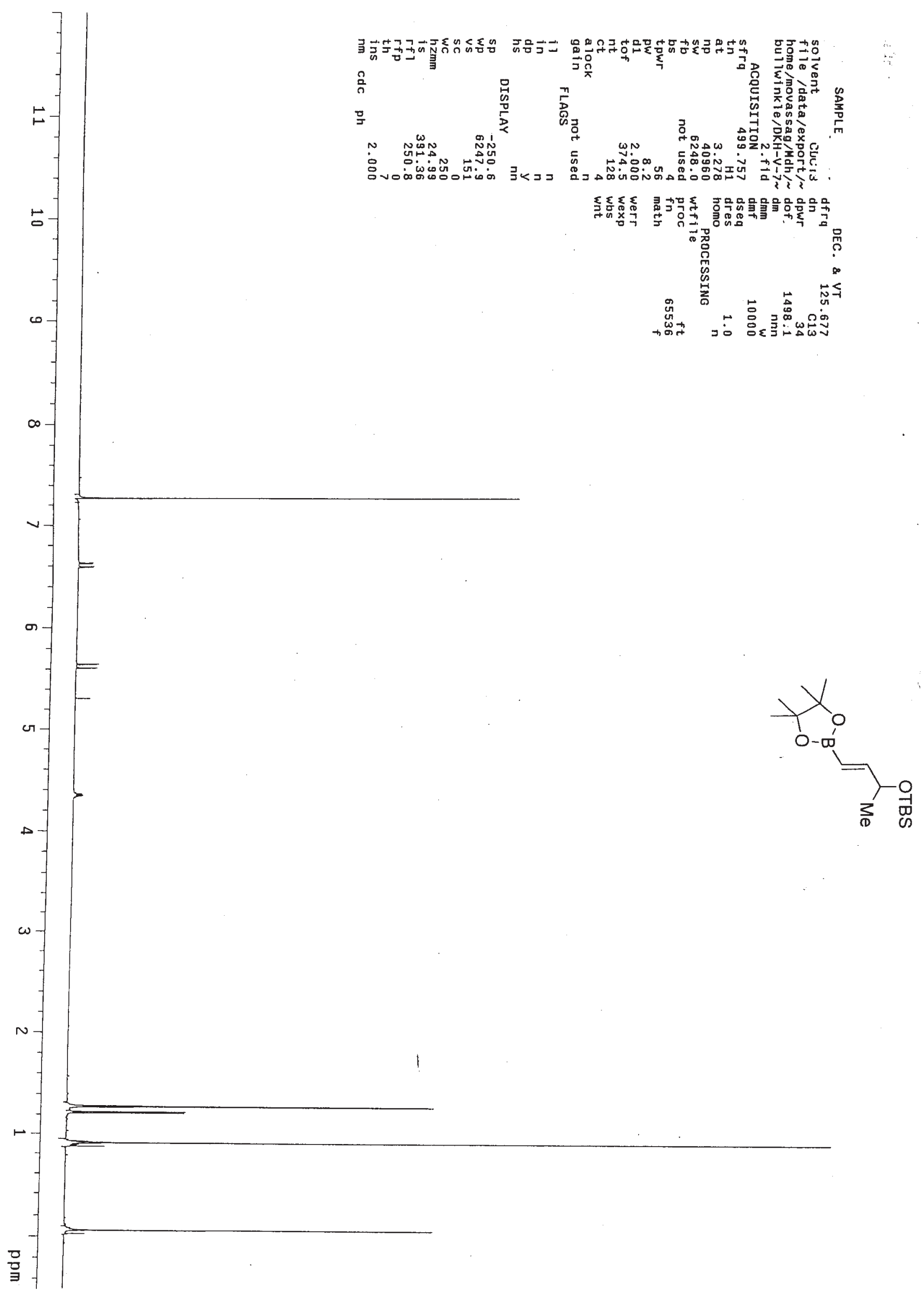




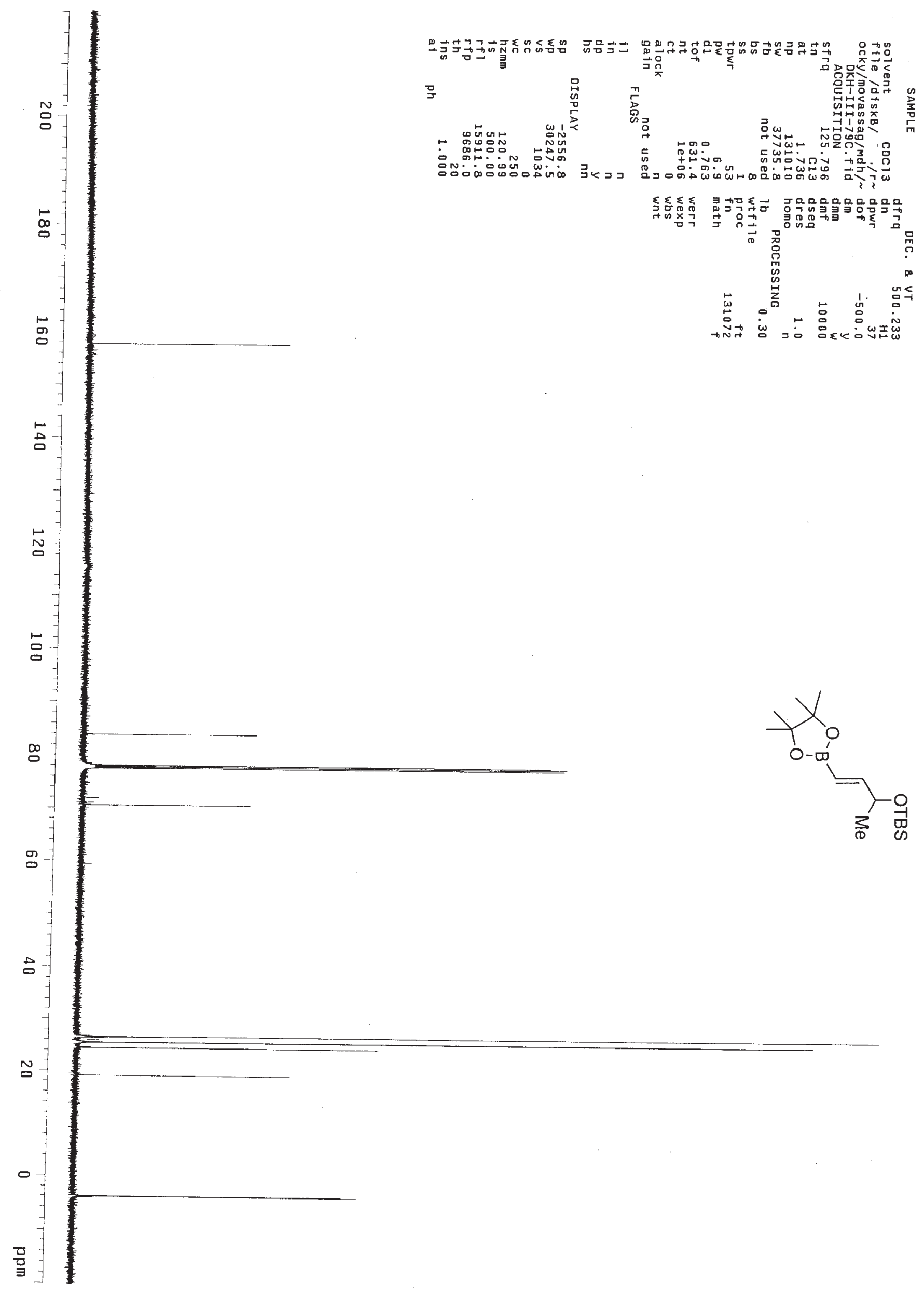



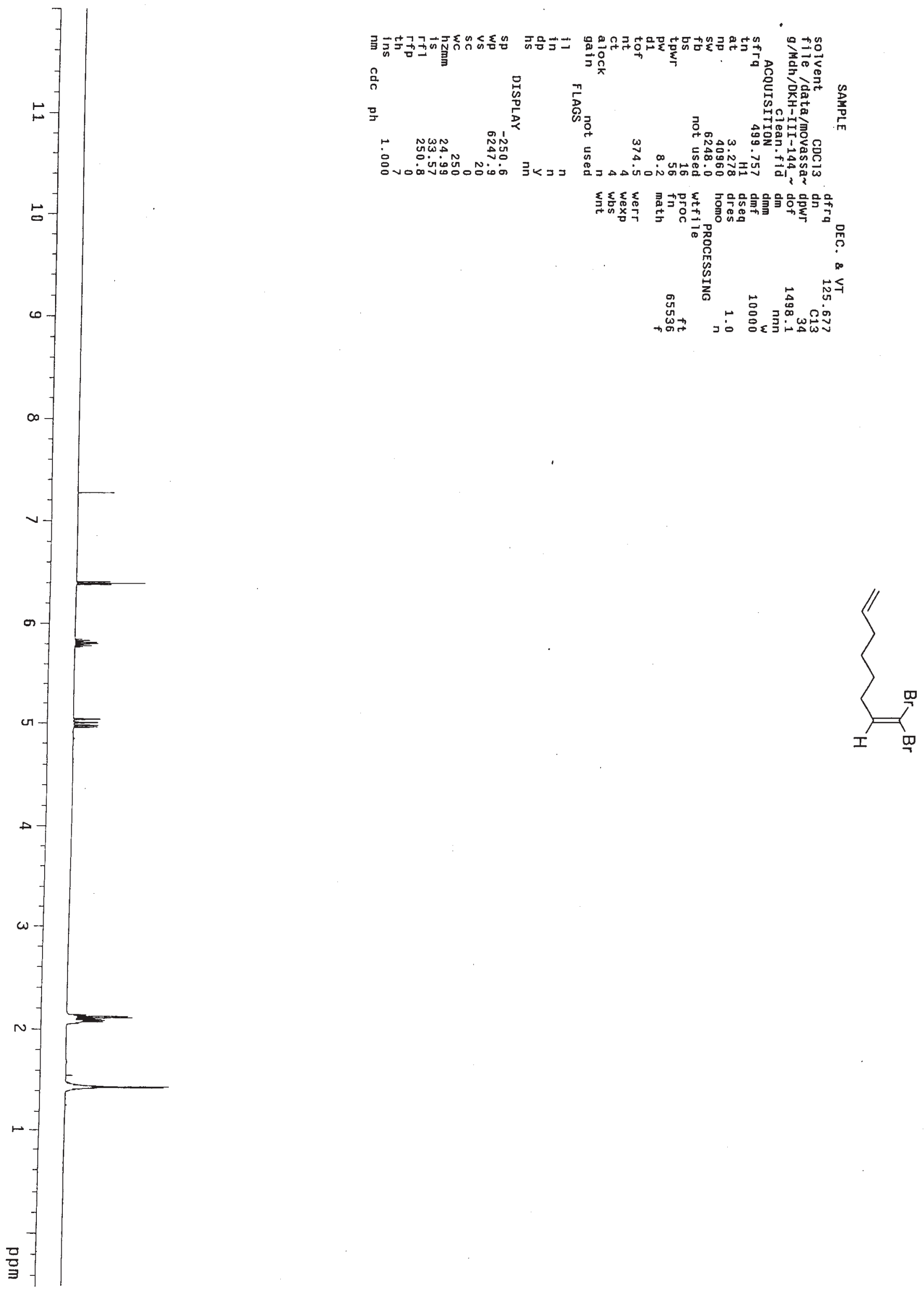


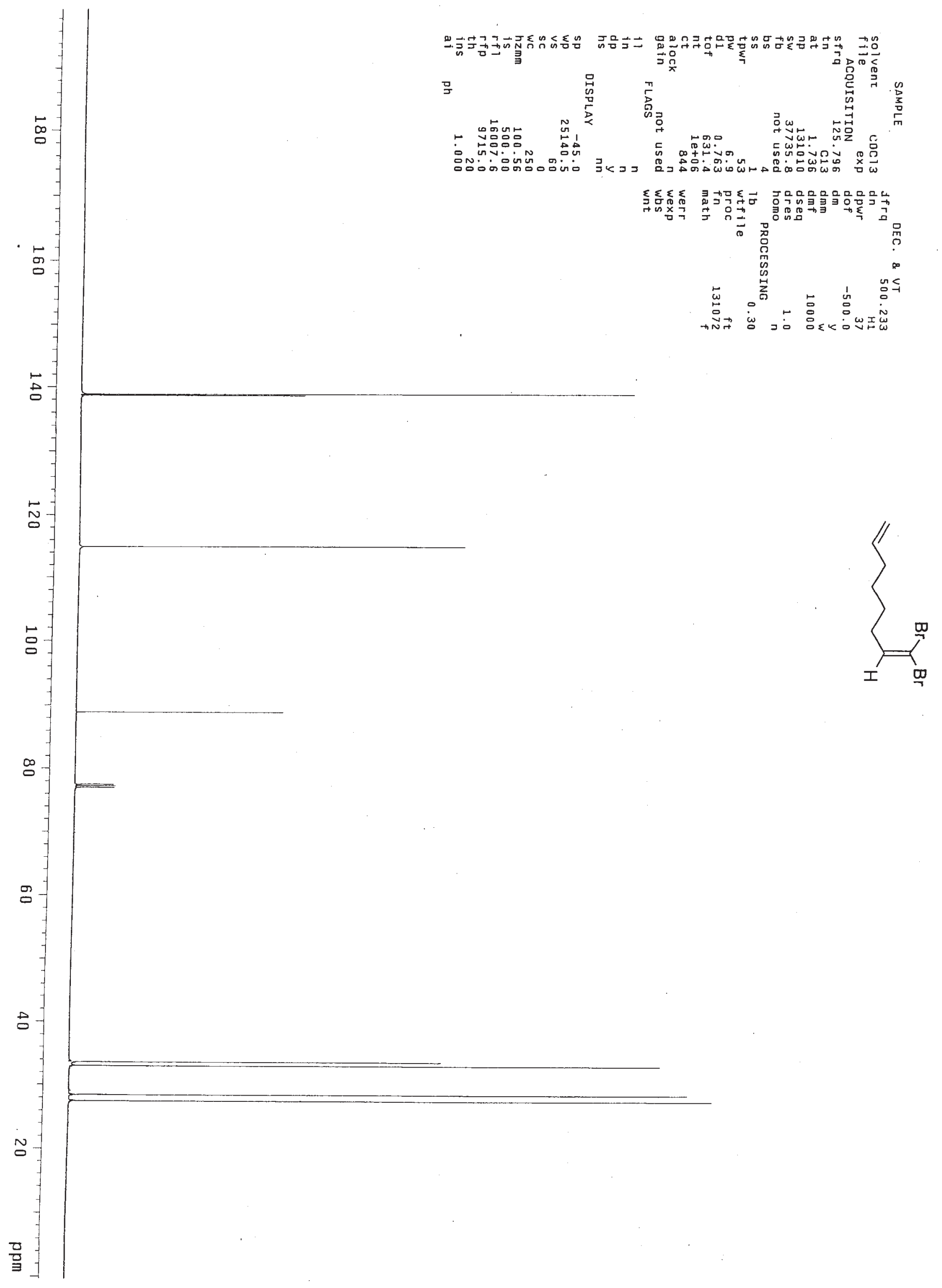




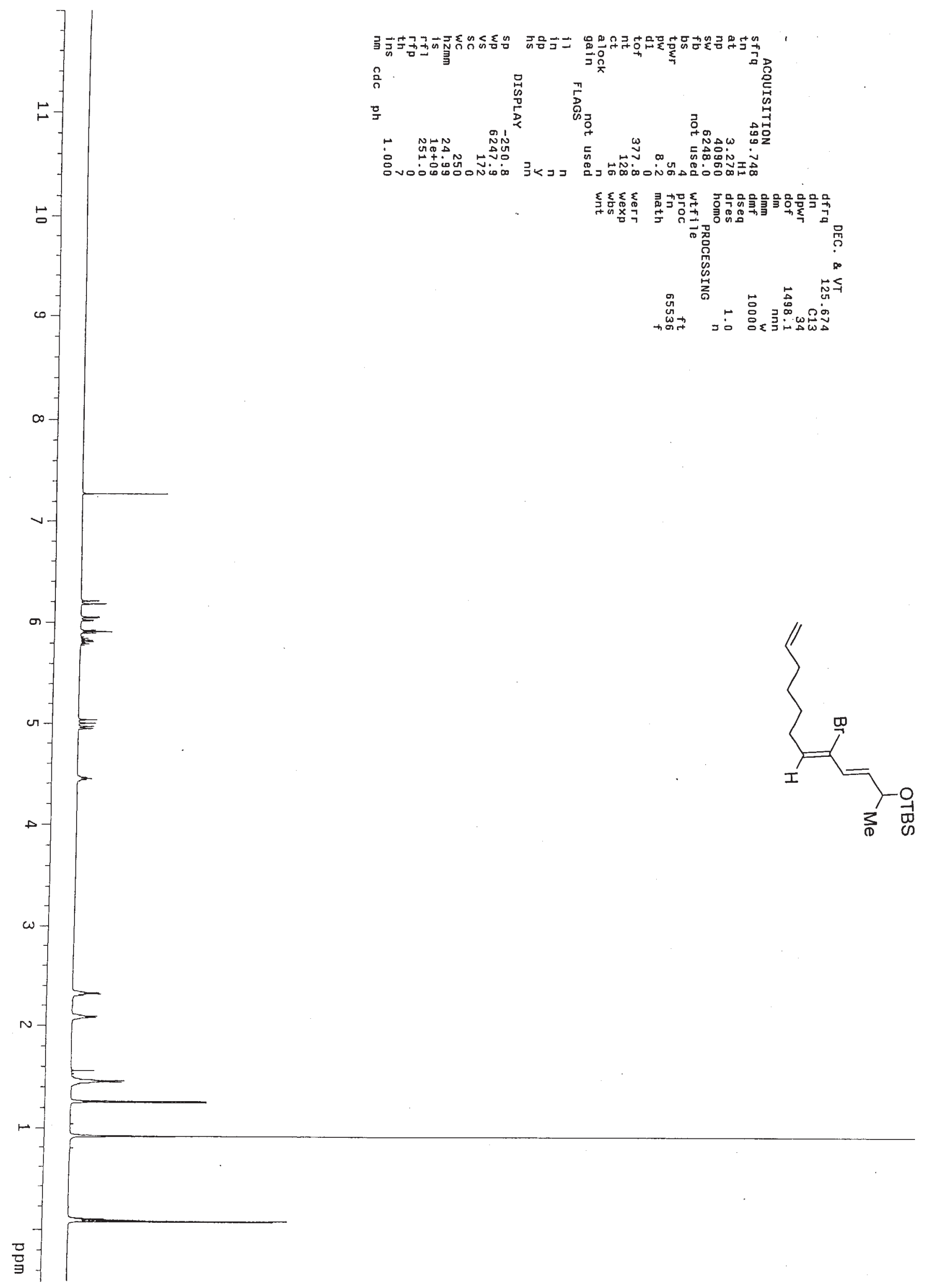




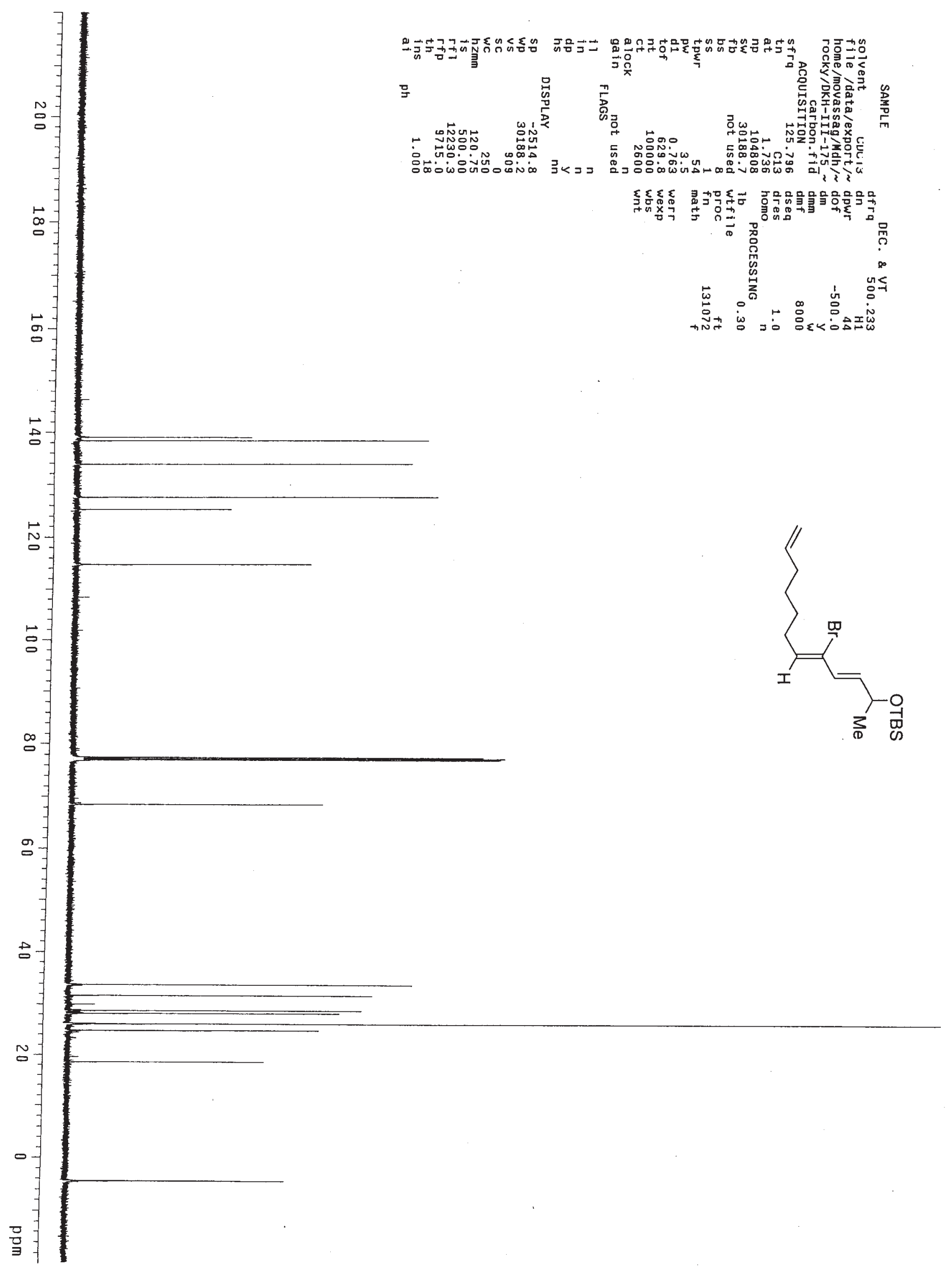




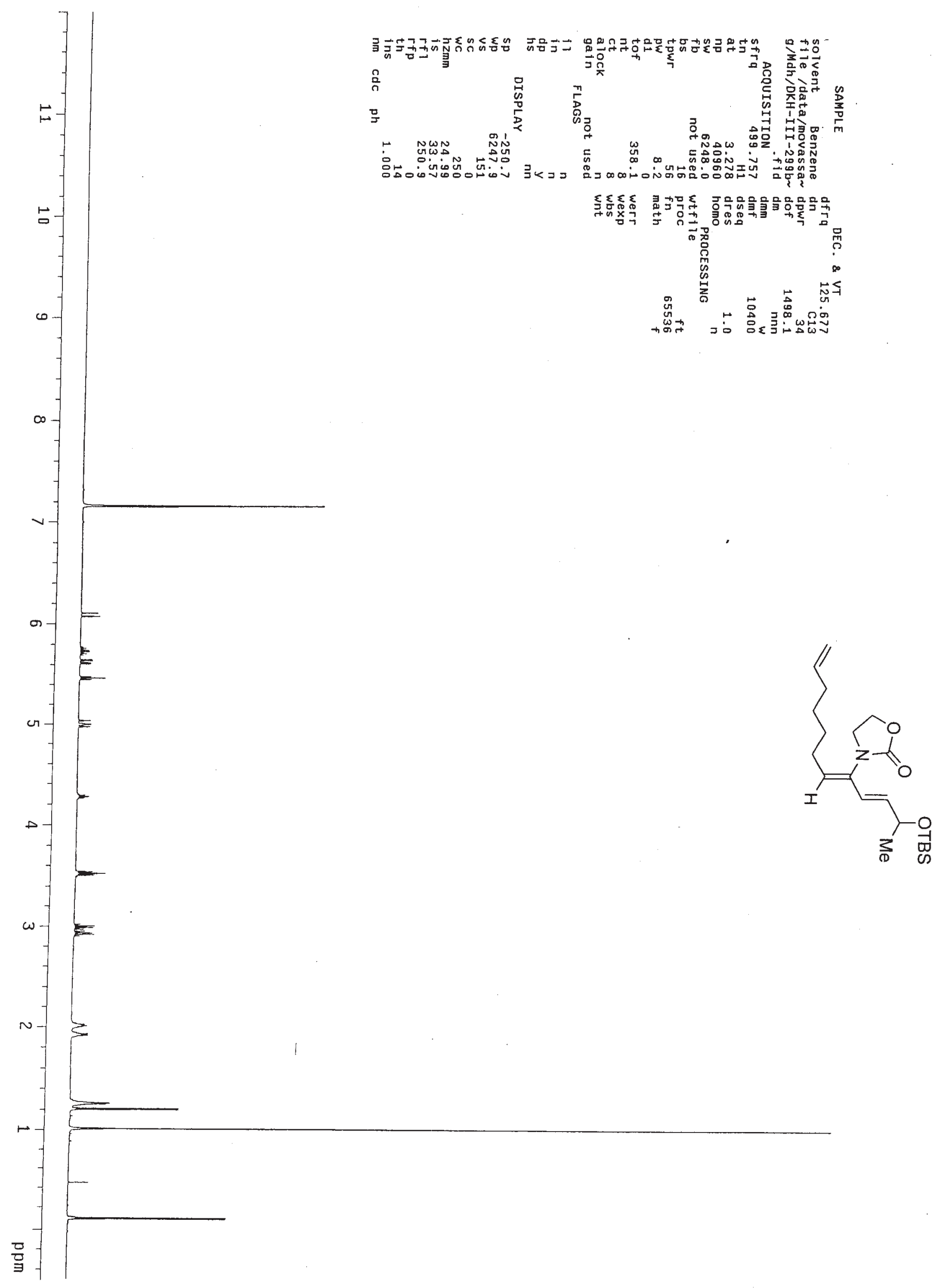




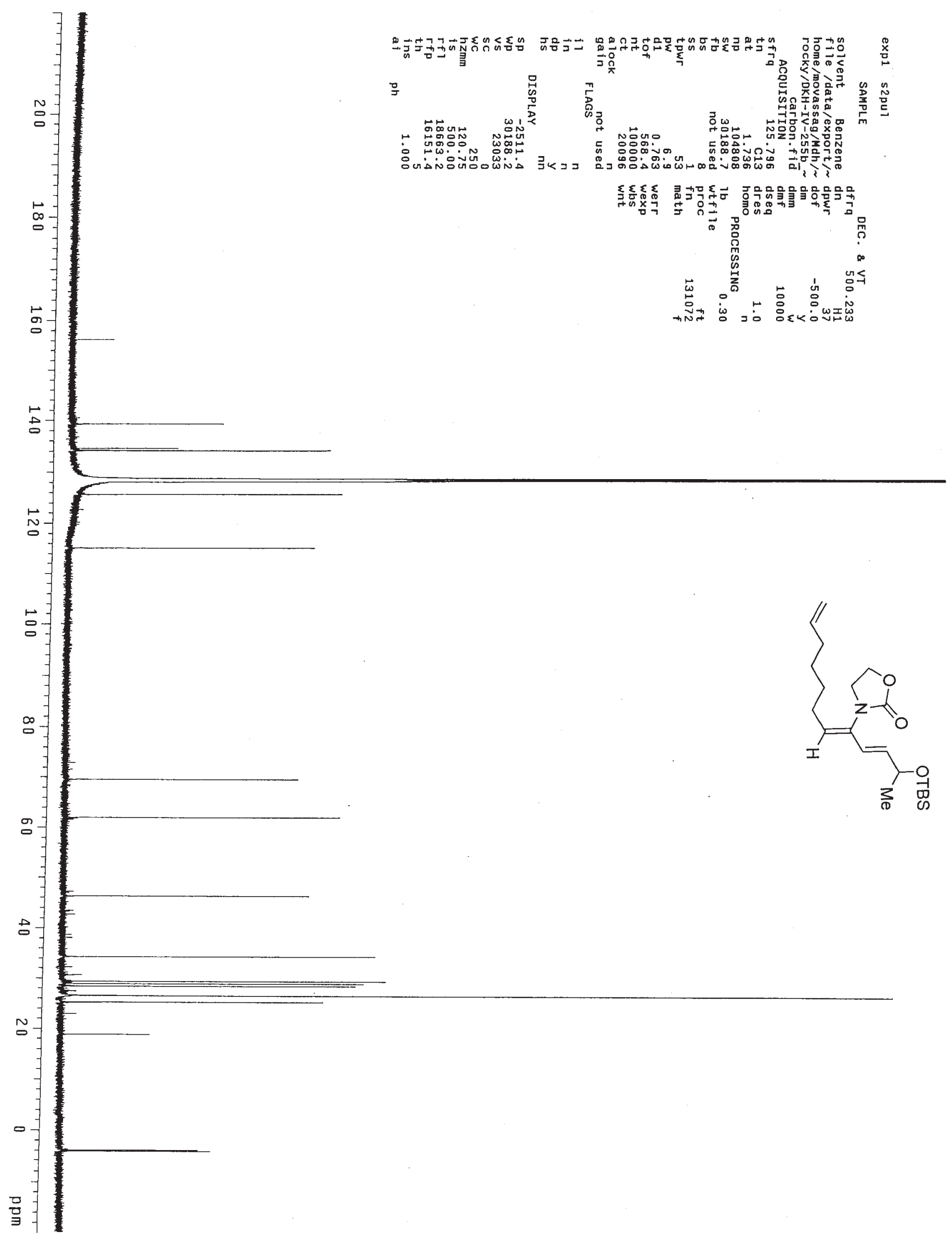




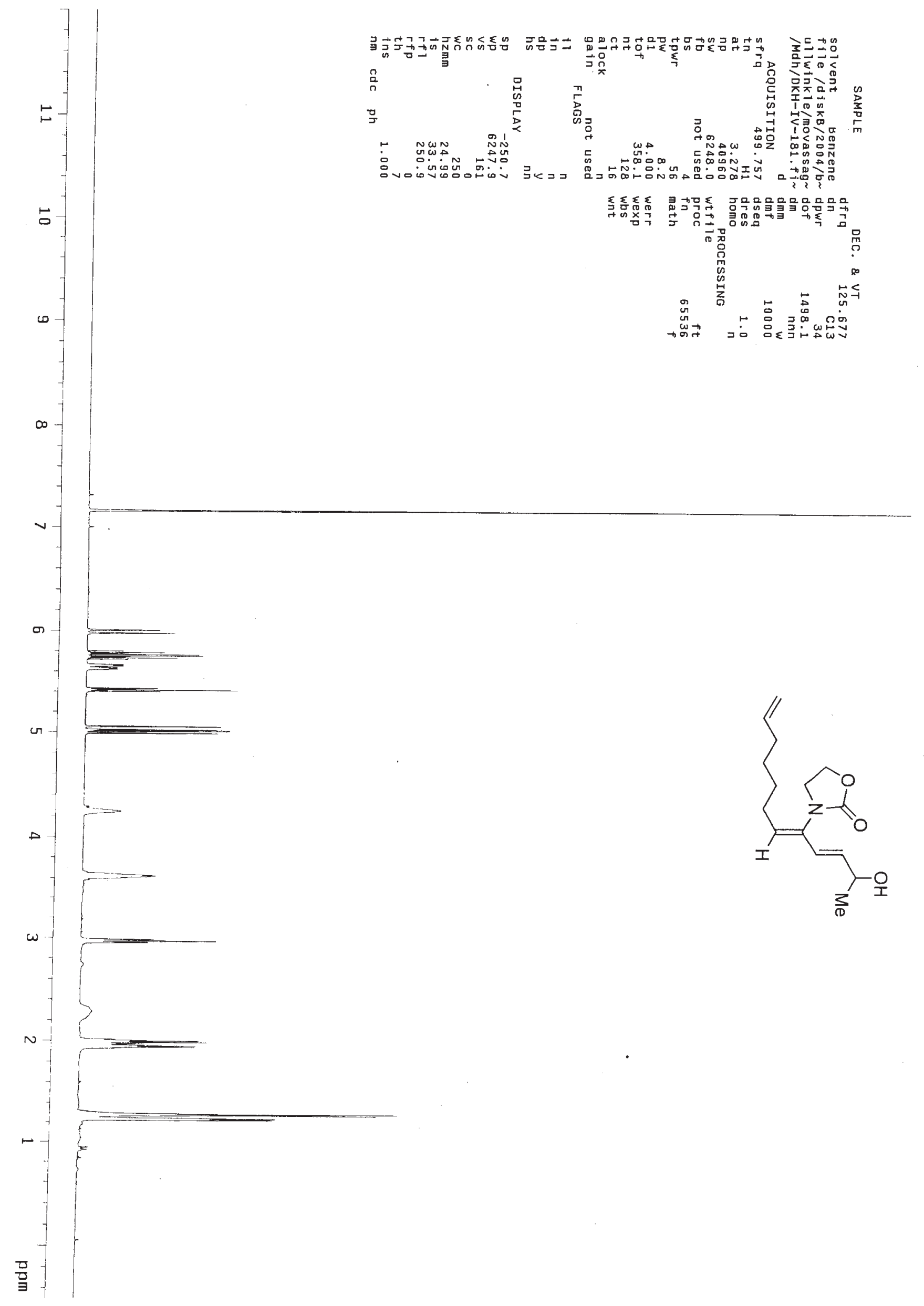




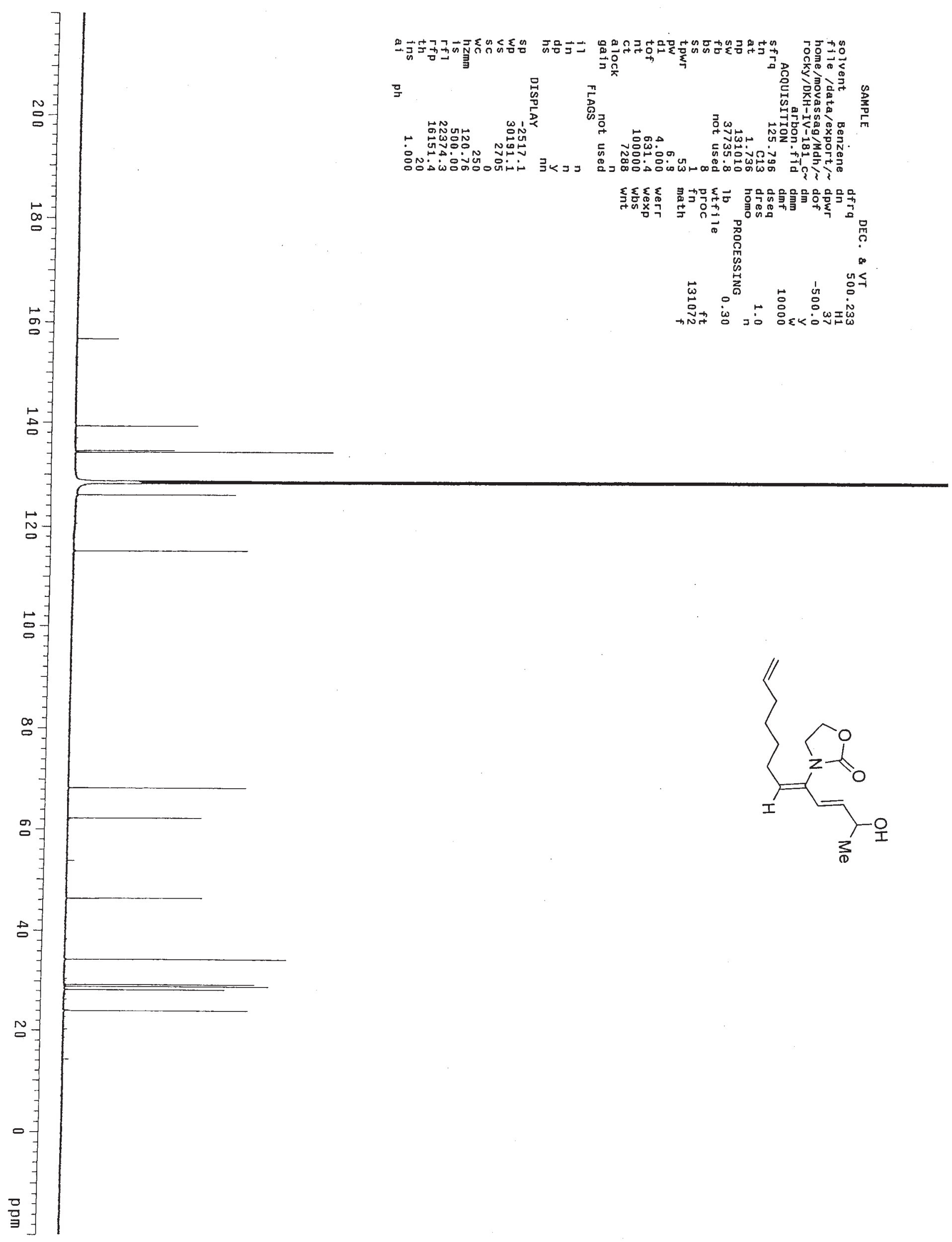




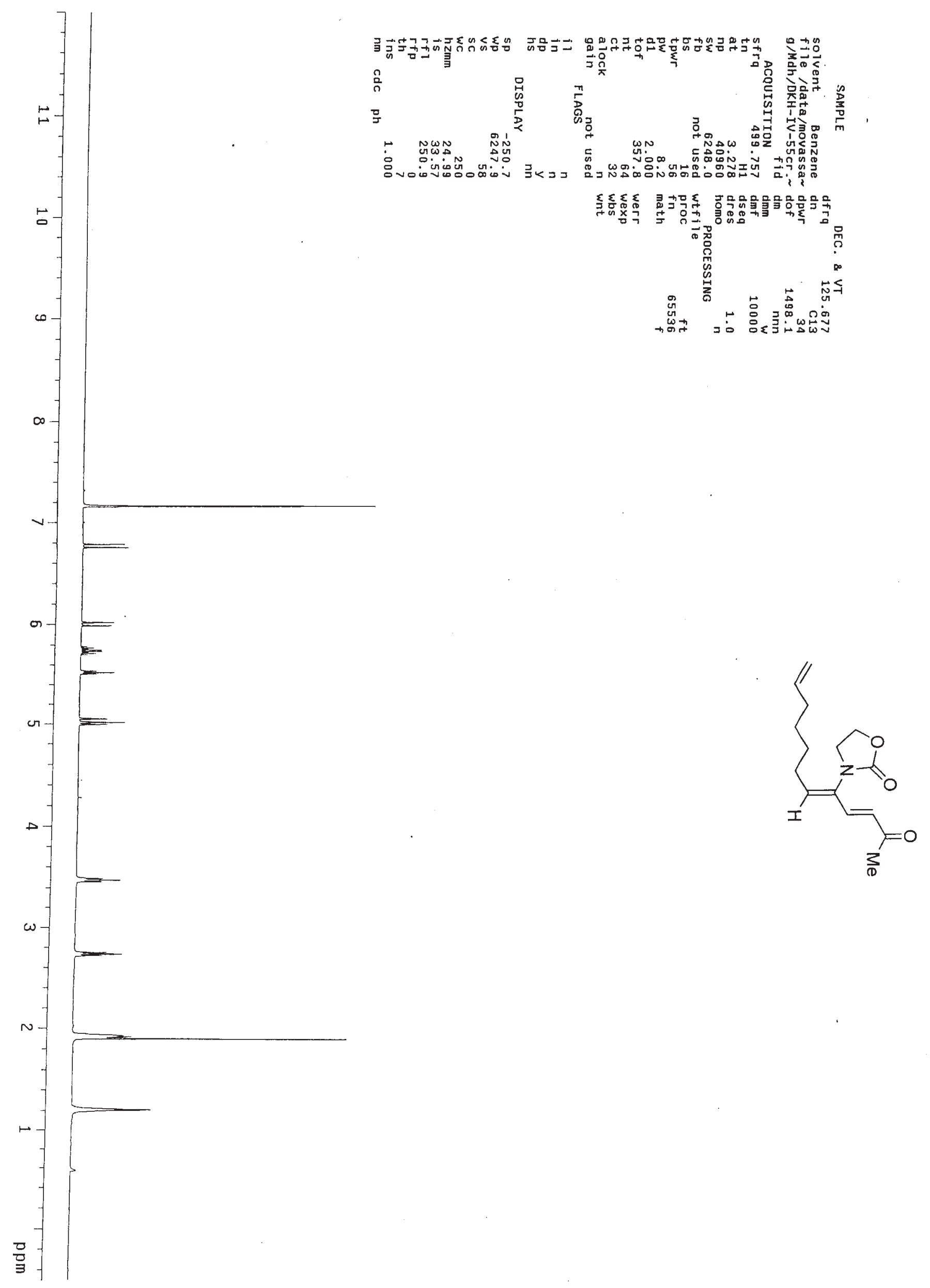




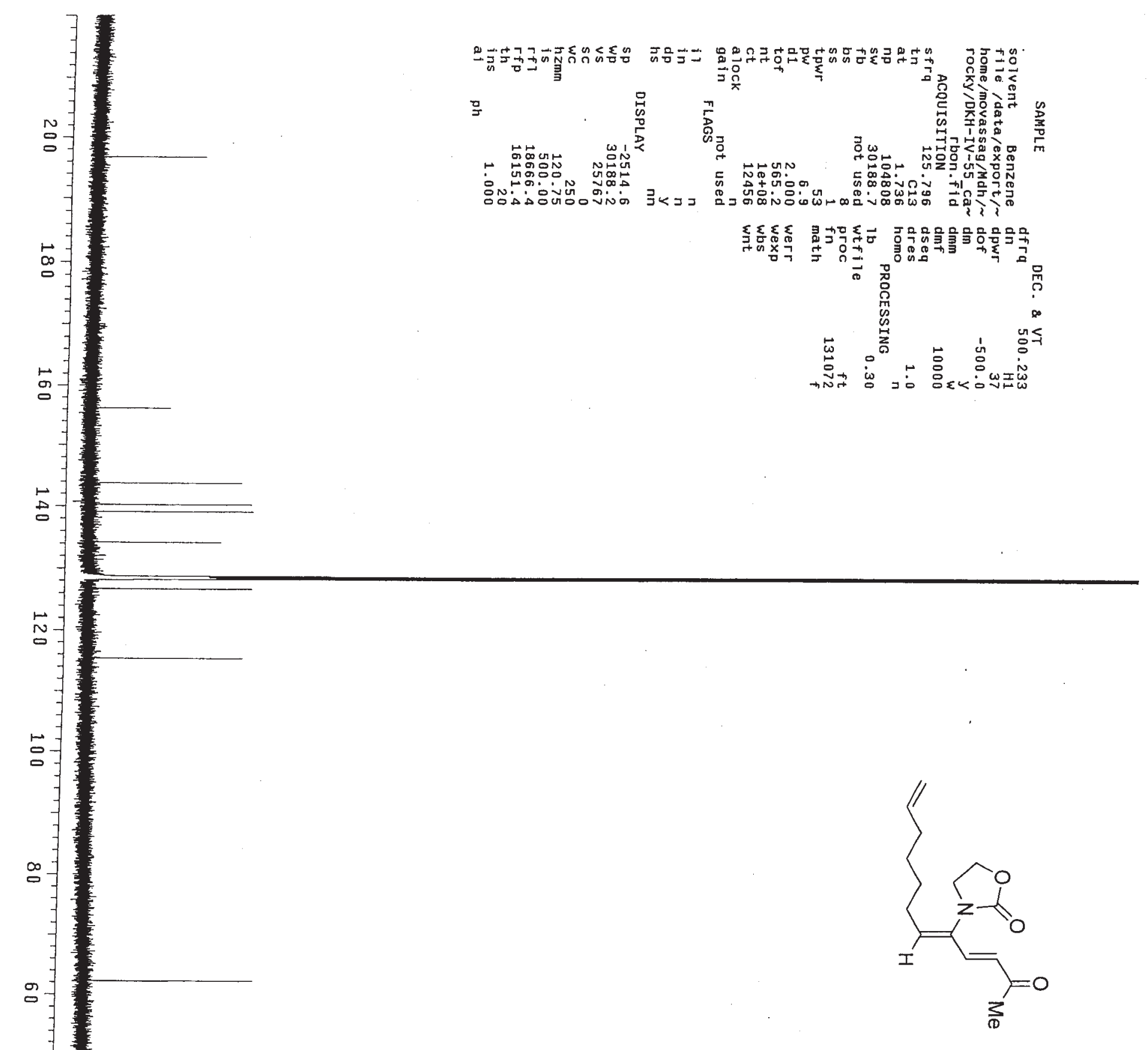




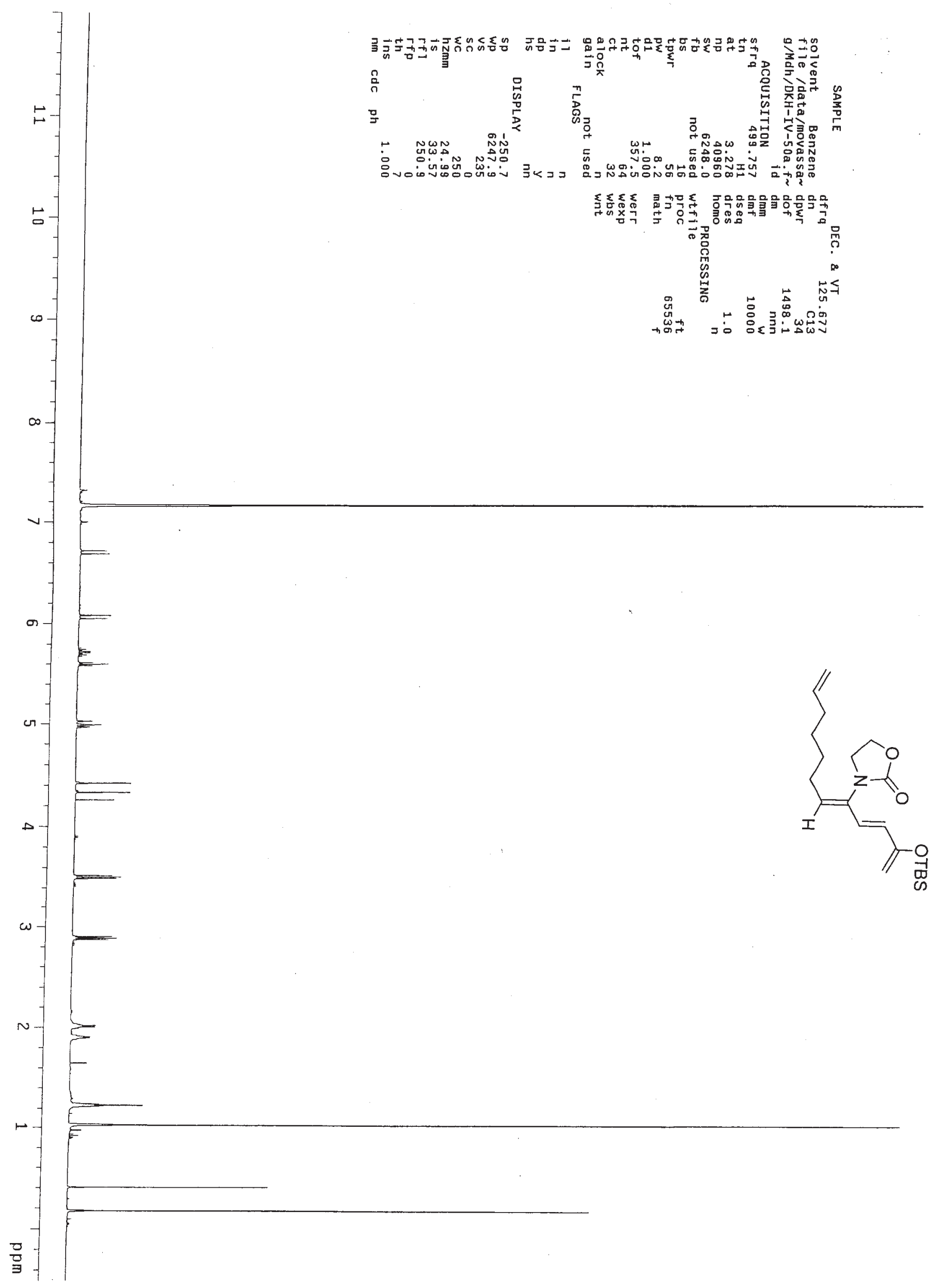




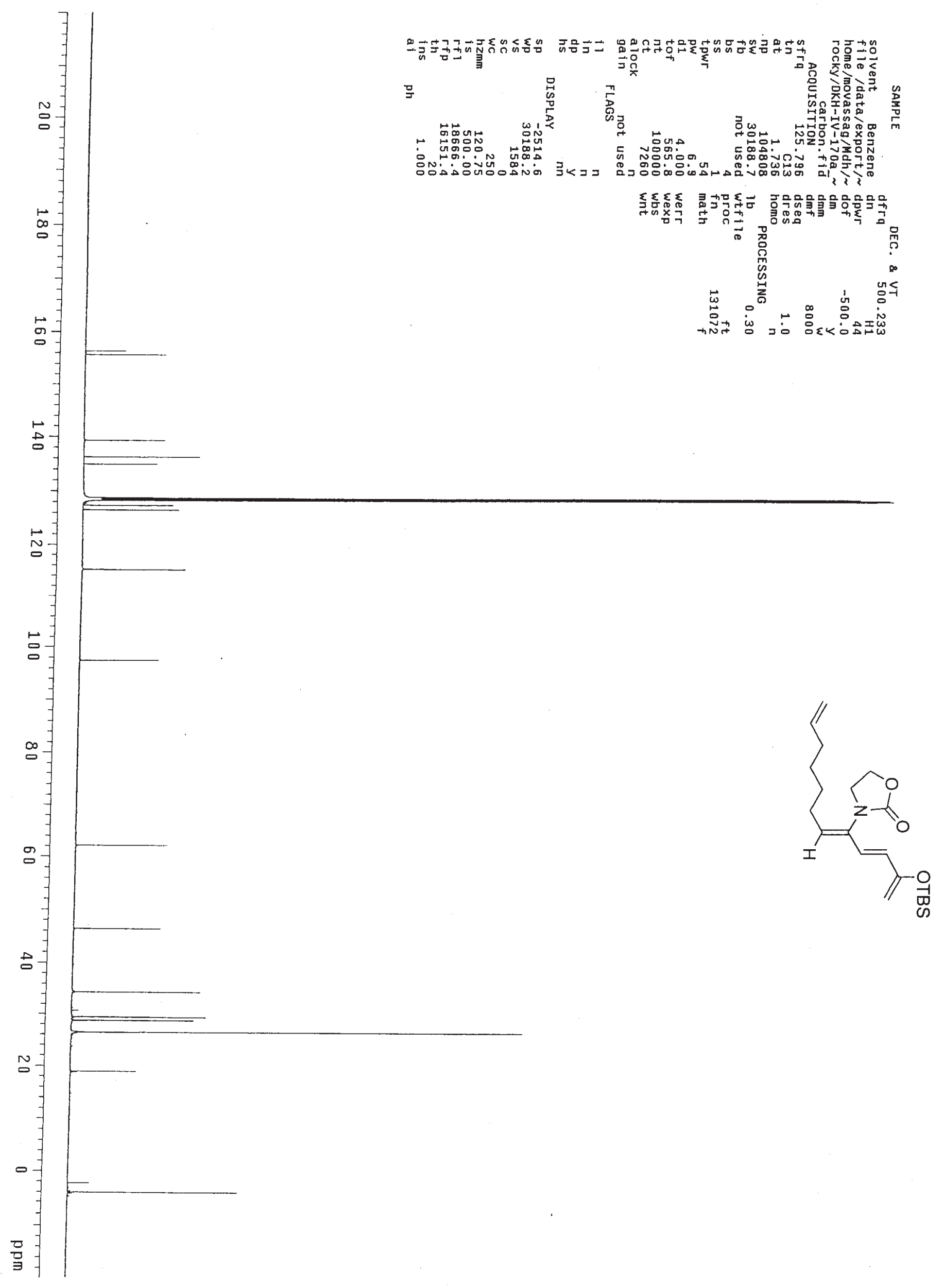




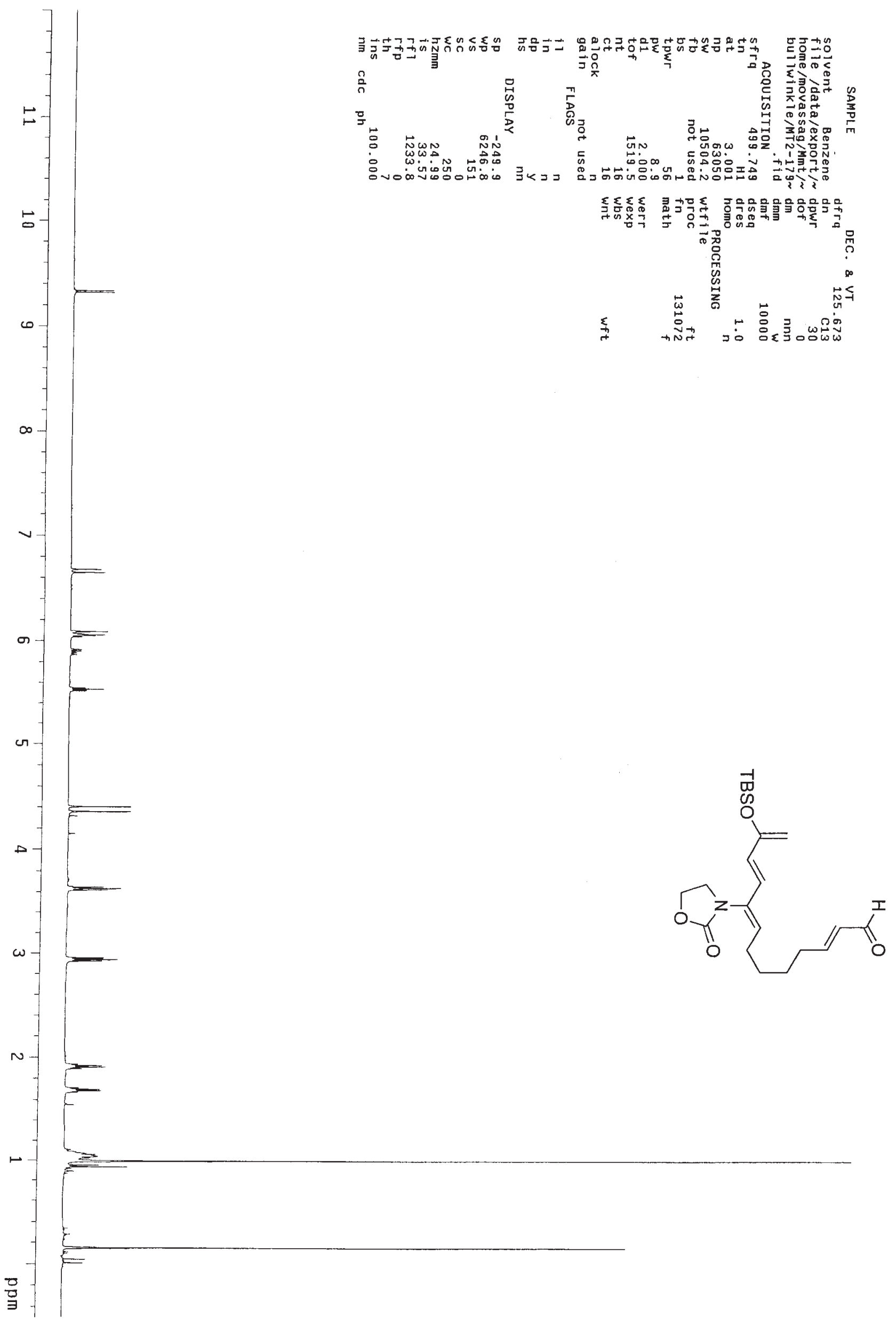




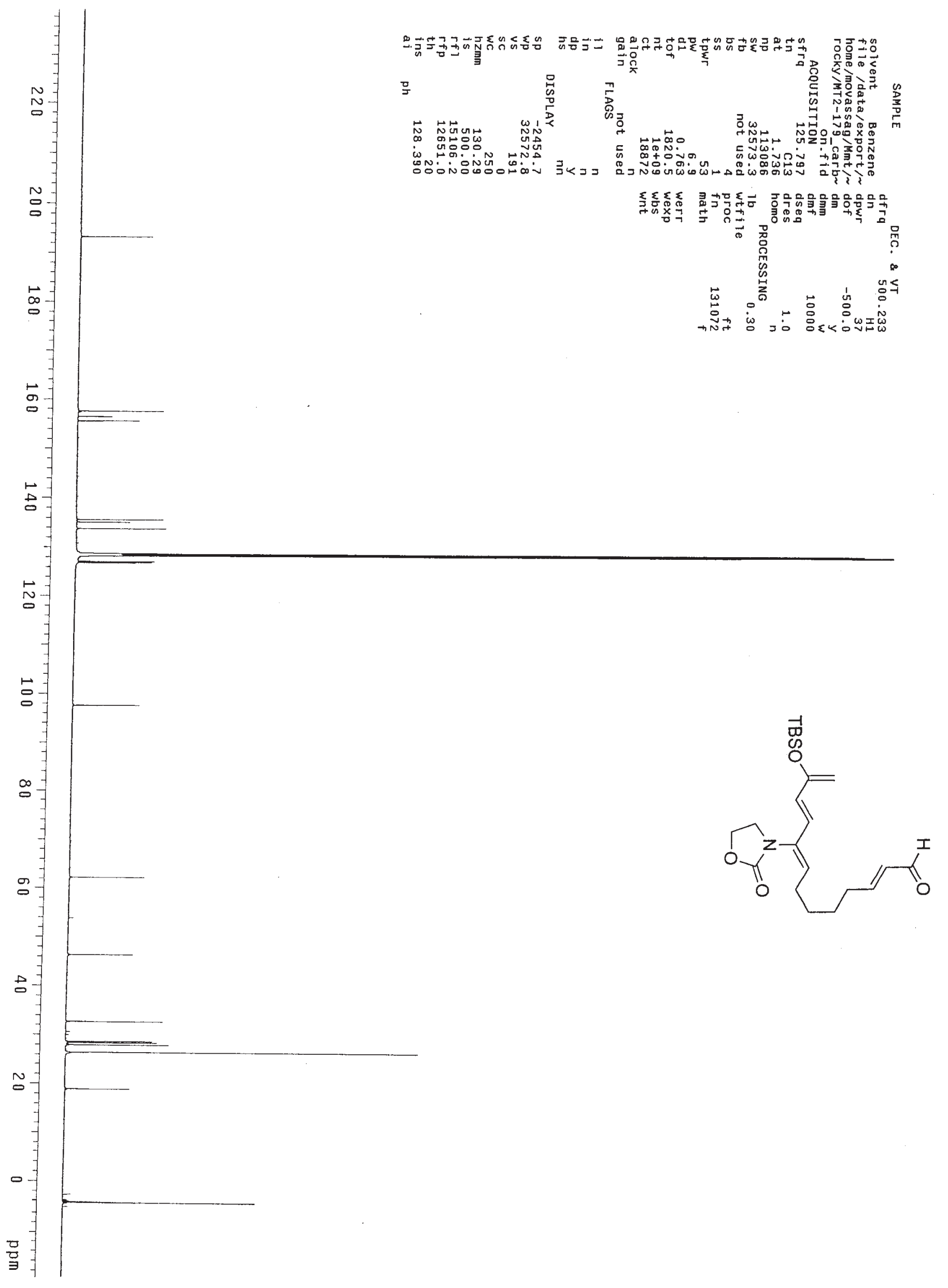




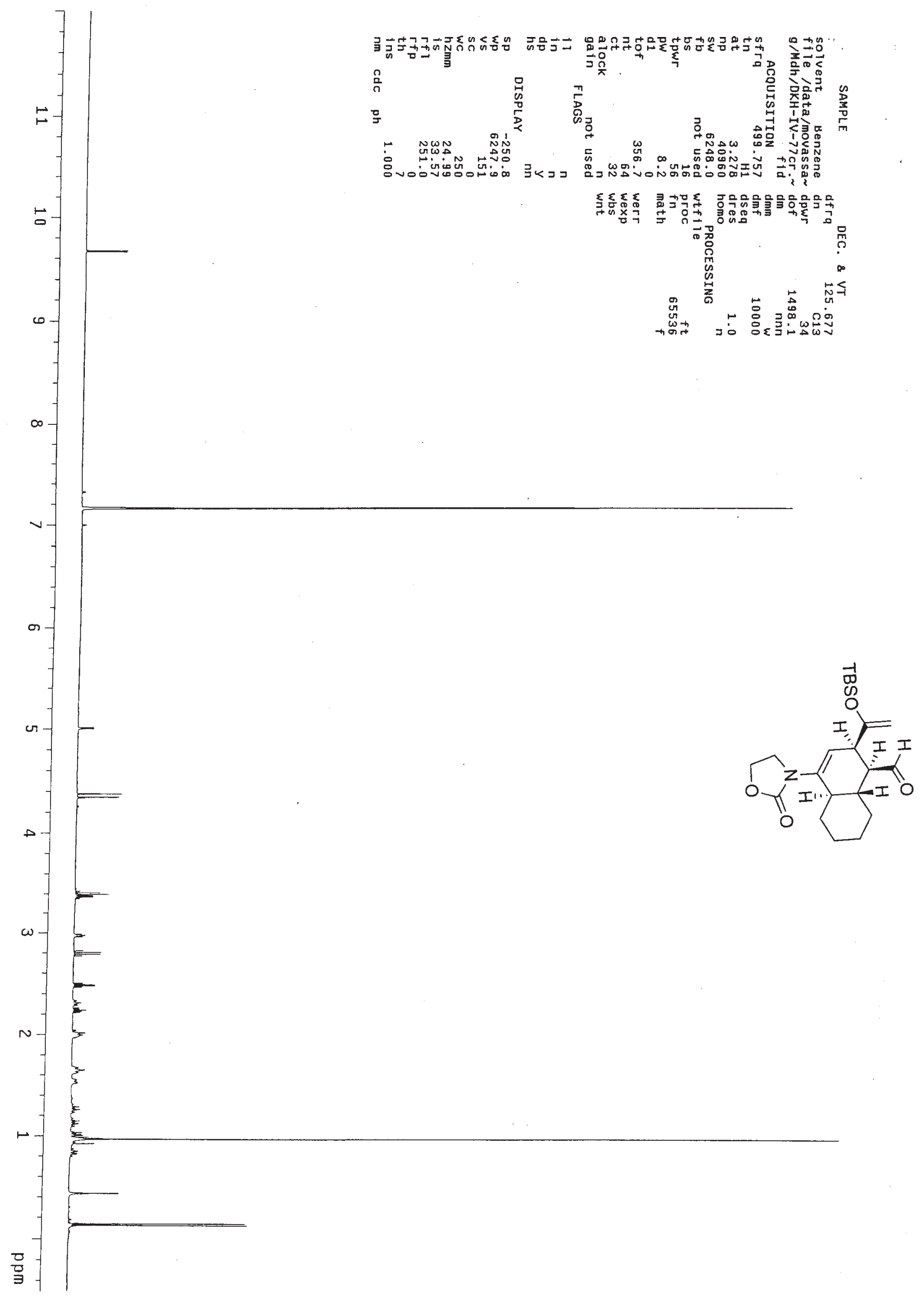




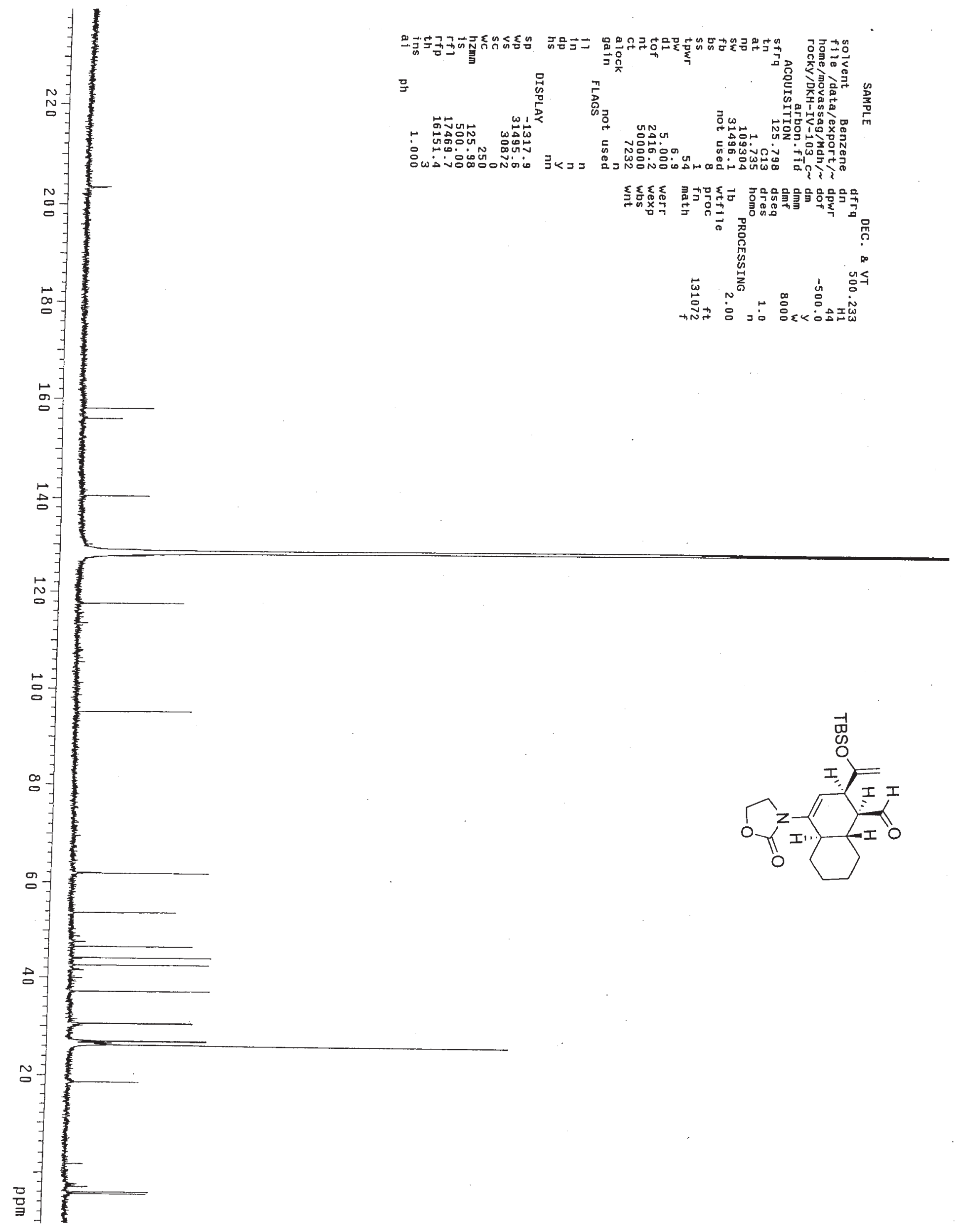




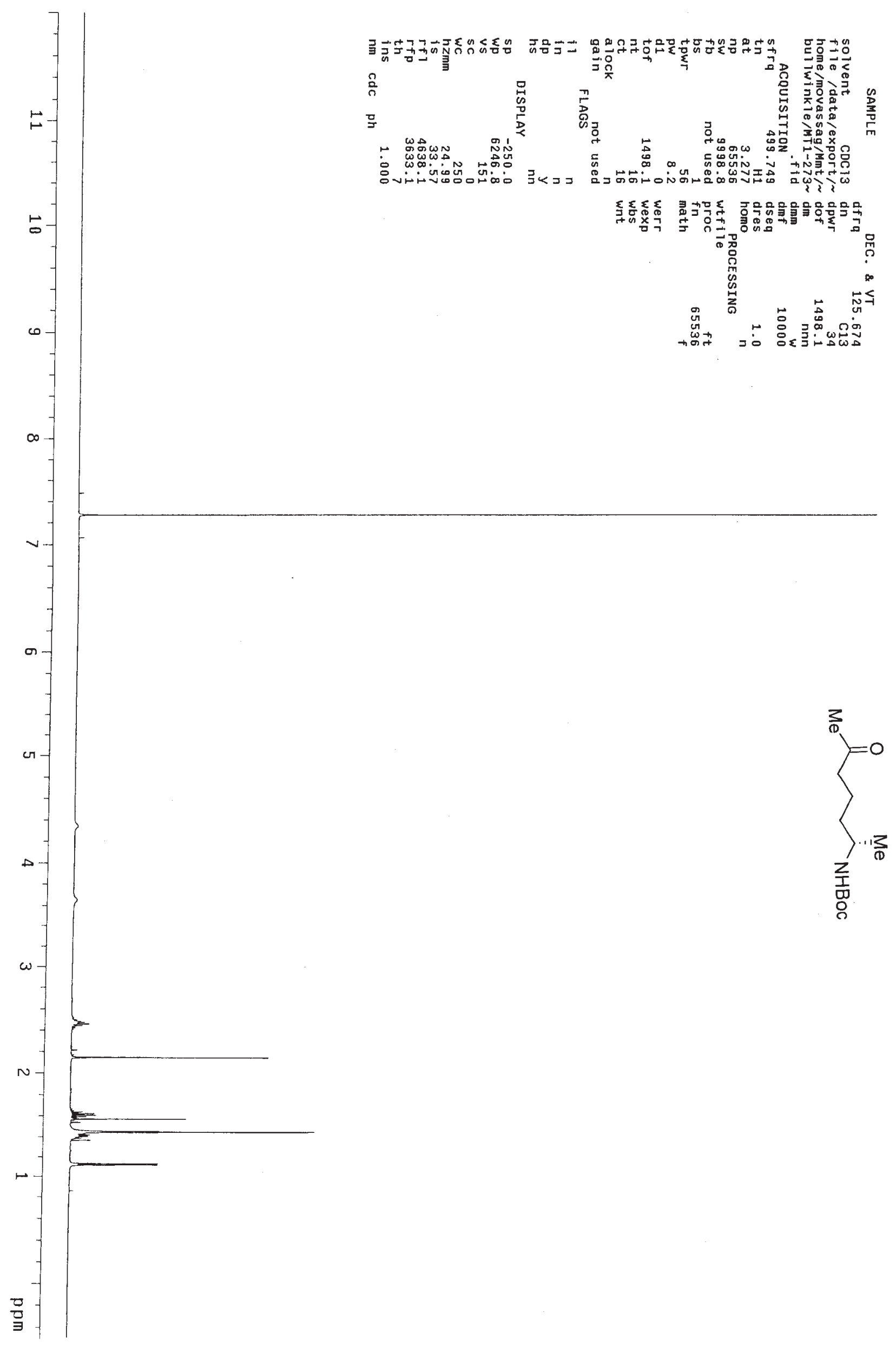




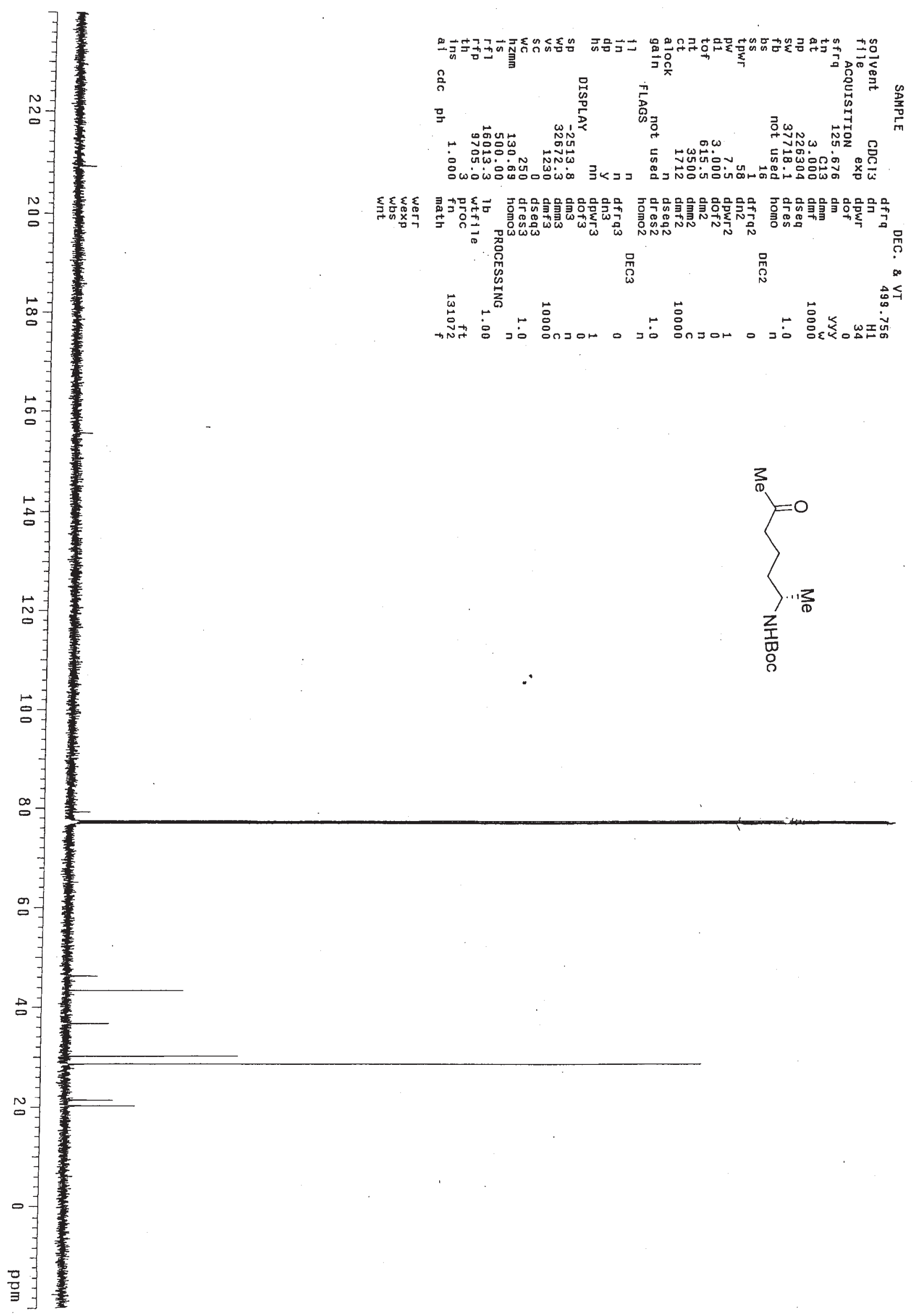




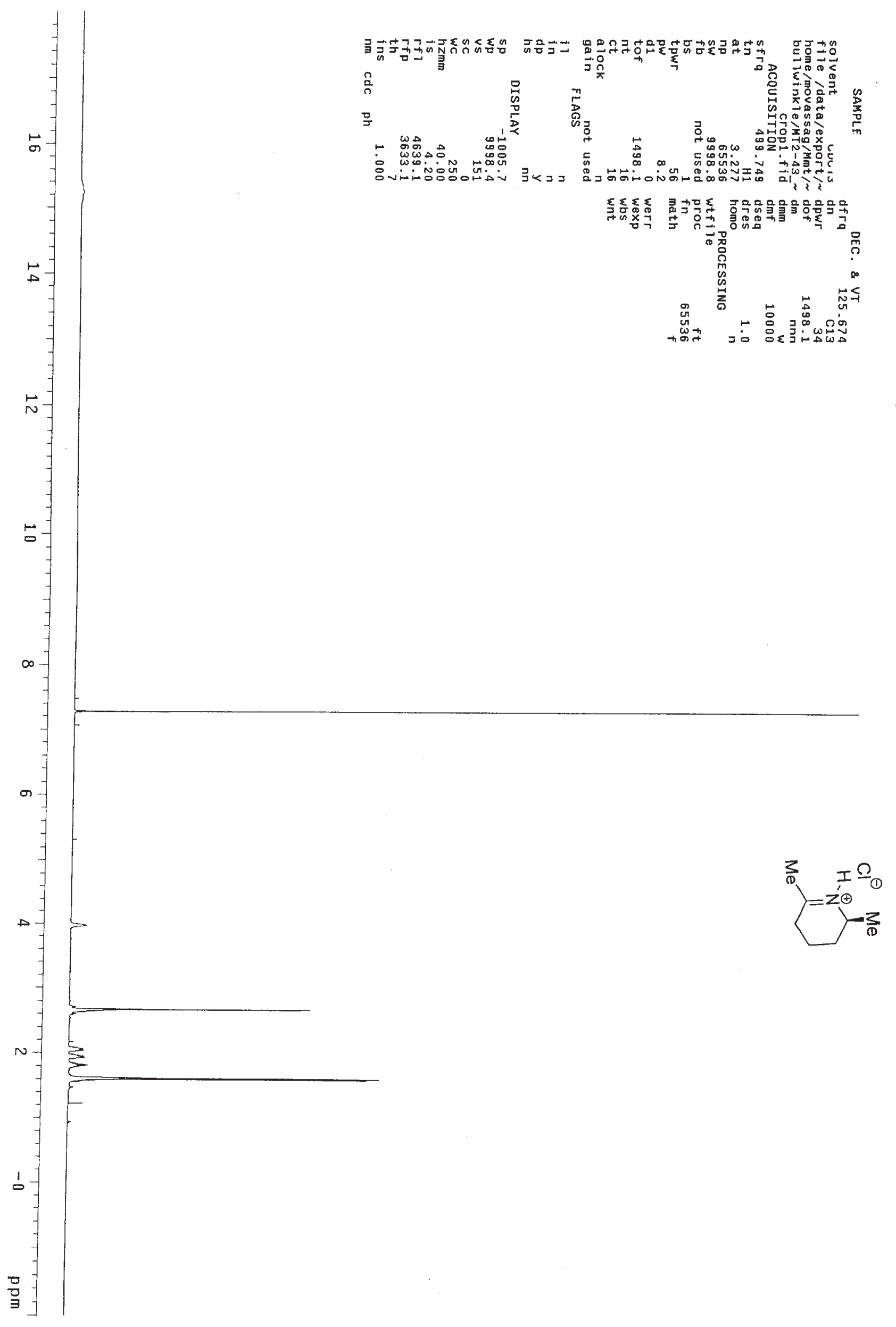




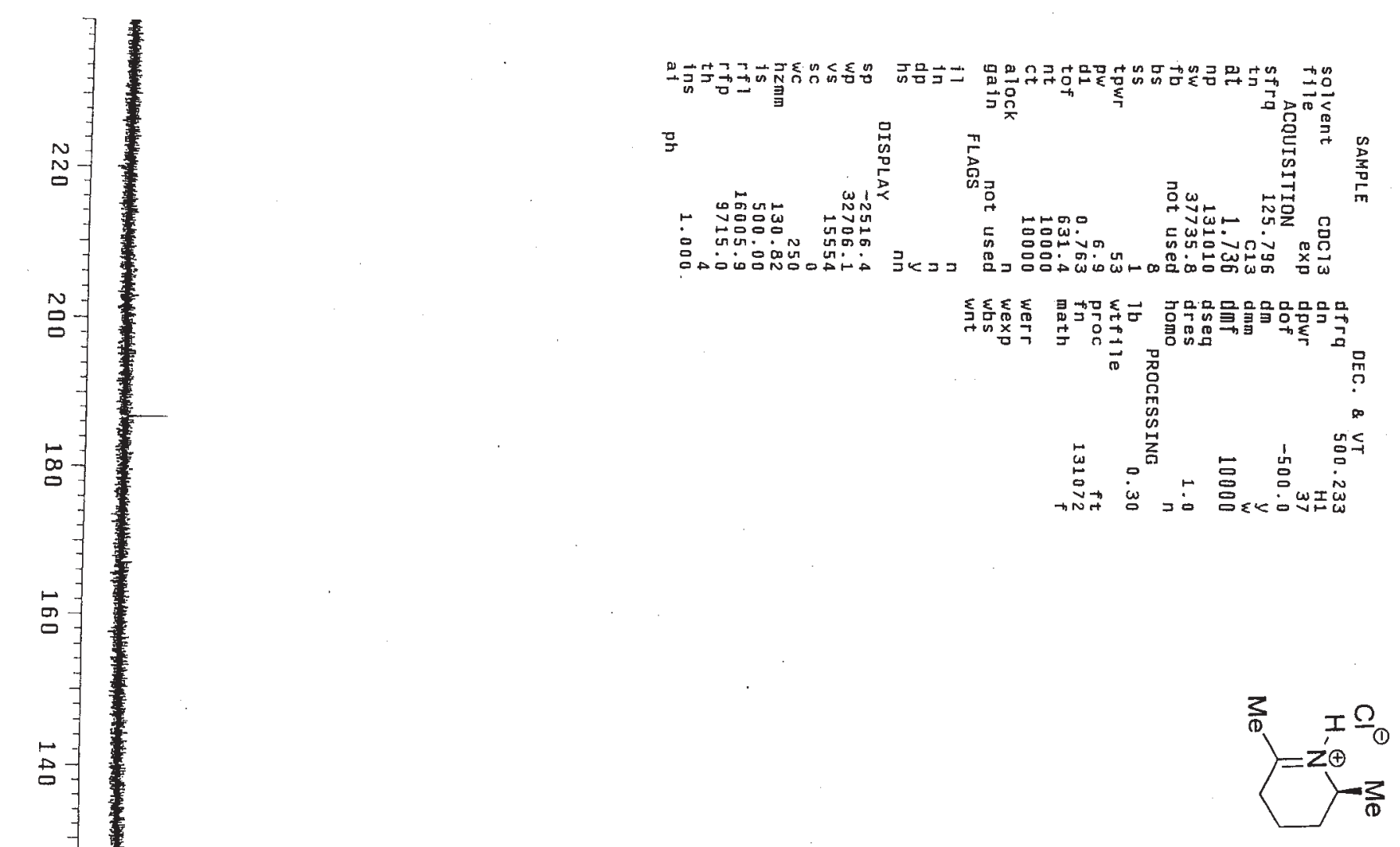




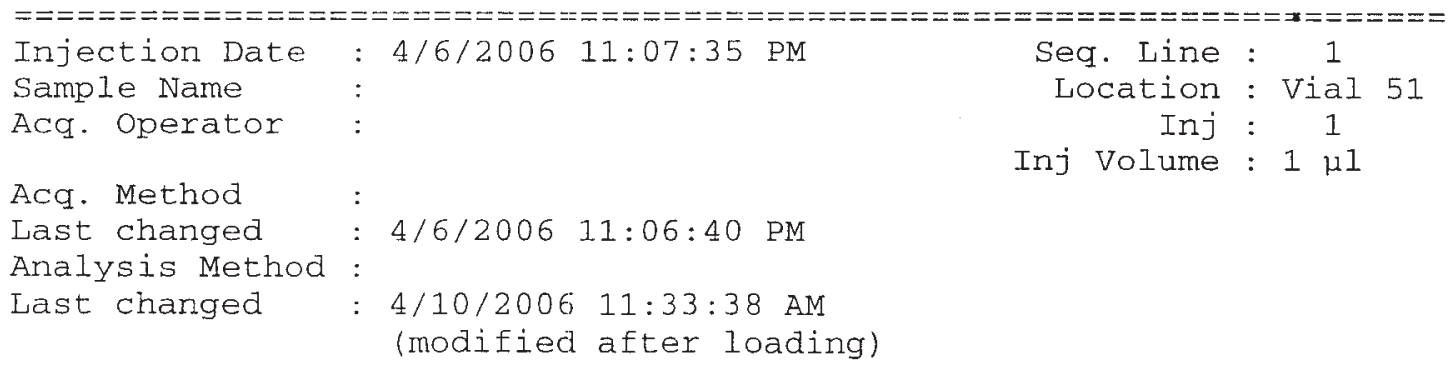

Acq. Method

Last changed

Analysis Method :

Last changed

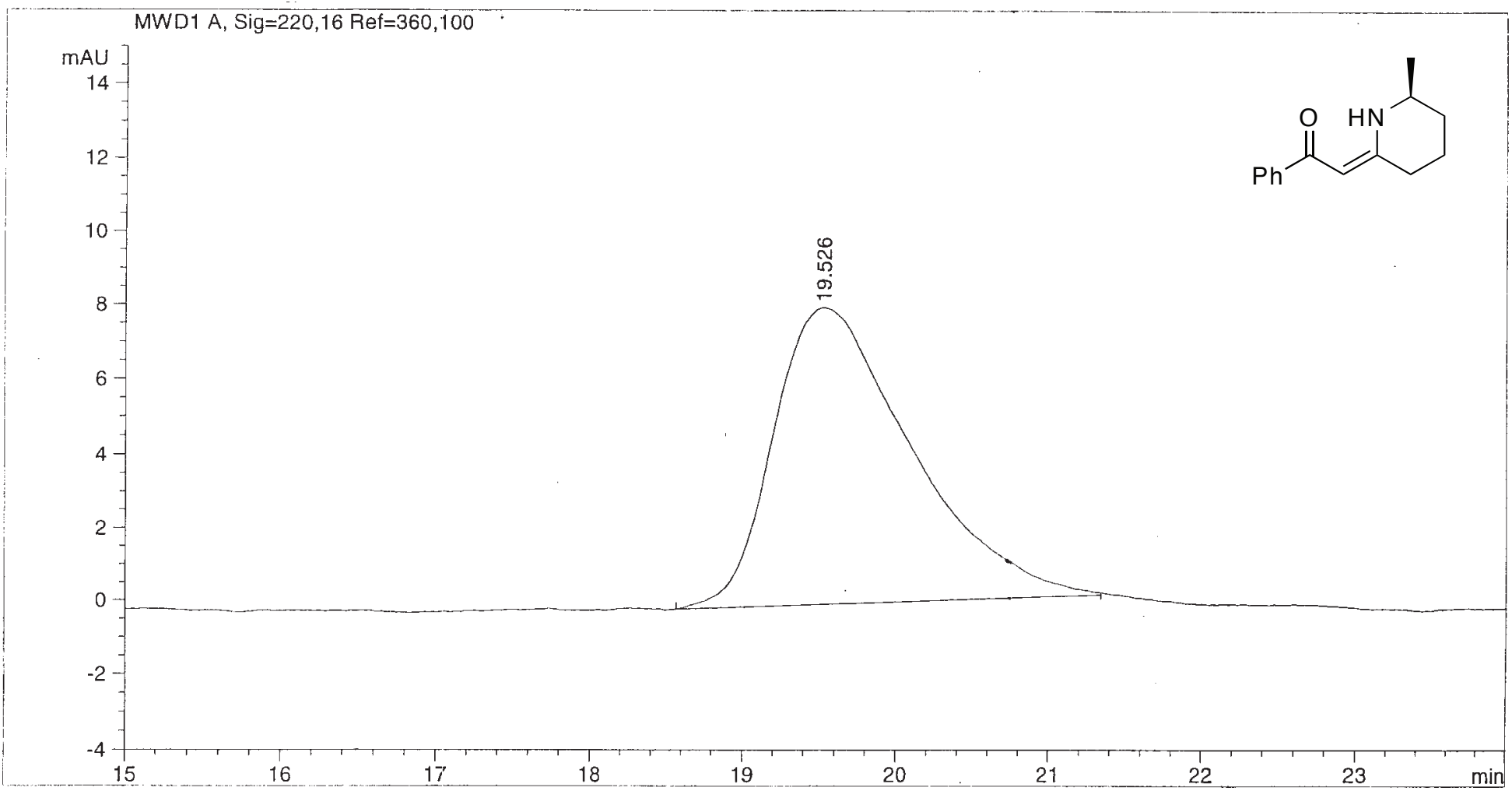

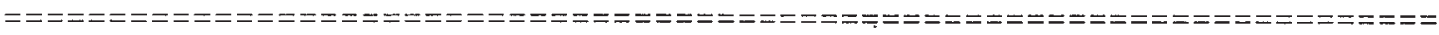
Area Percent Report

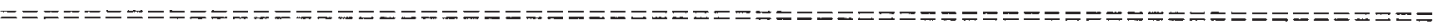

Sorted By

Multiplier

Dilution

Signal

Use Multiplier \& Dilution Factor with ISTDs

Signal 1: MWD1 A, Sig=220,16 Ref=360,100

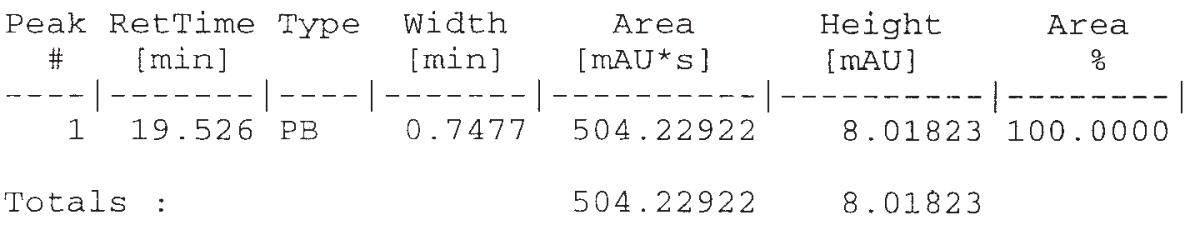

Results obtained with enhanced integrator!

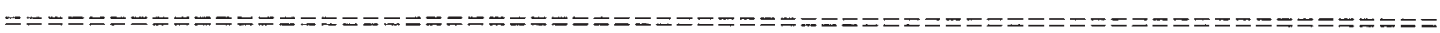

*** End of Report *** 


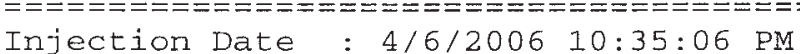

Sample Name

Acq. Operator

Acq. Method :

Last changed : $416 / 2006$ 10:35:16 PM

Analysis Method :

Last changed : 4/10/2006 11:36:59 AM

(modified after loading)
S, S Whelk-O

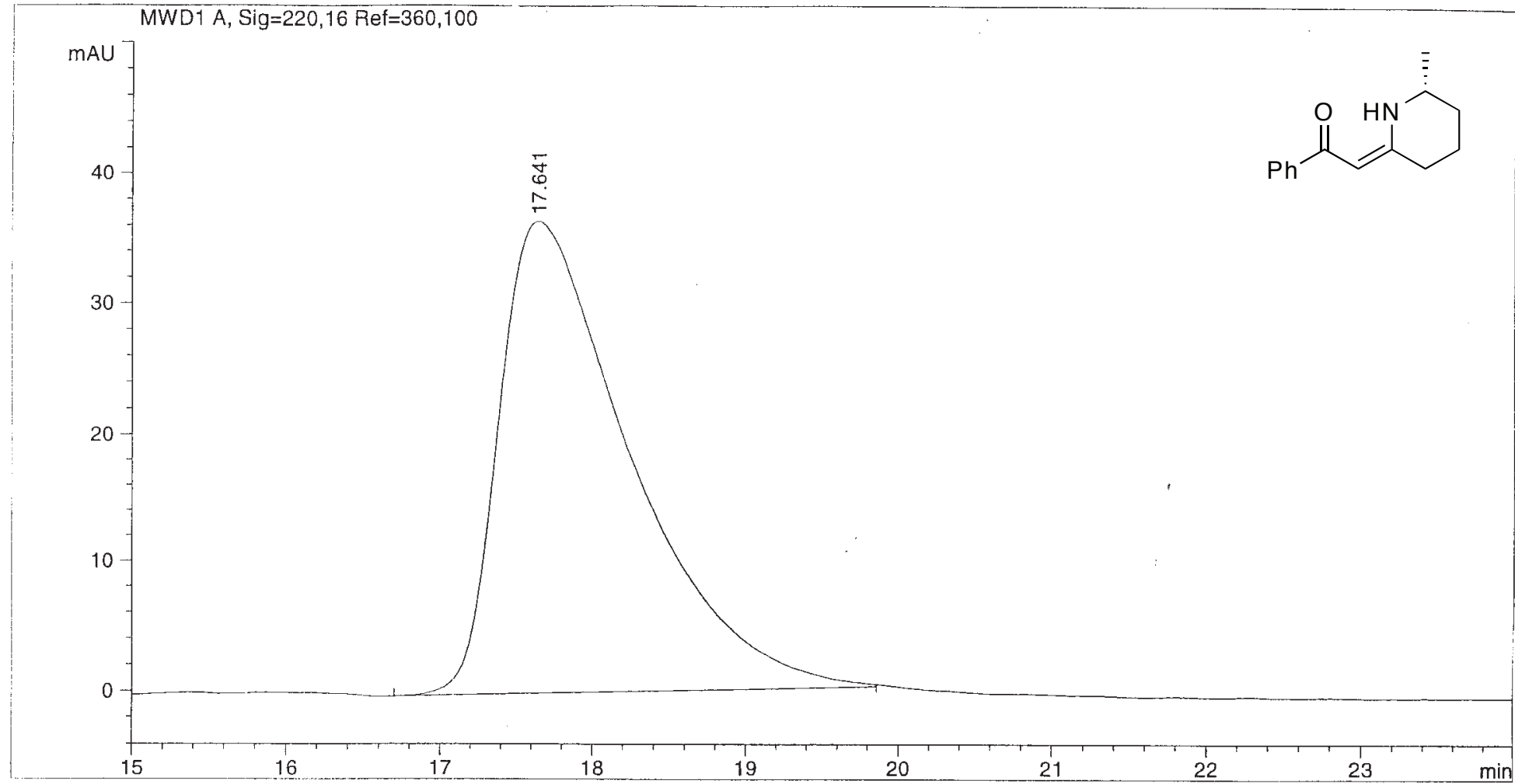

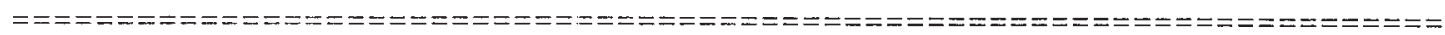

Area Percent Report

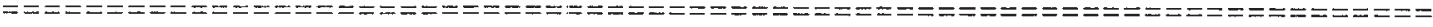

Sorted By

Multiplier

Signal

Dilution

1.0000

1.0000

Use Multiplier \& Dilution Factor with ISTDS

Signal 1: MWD1 A, Sig=220,16 Ref=360,100

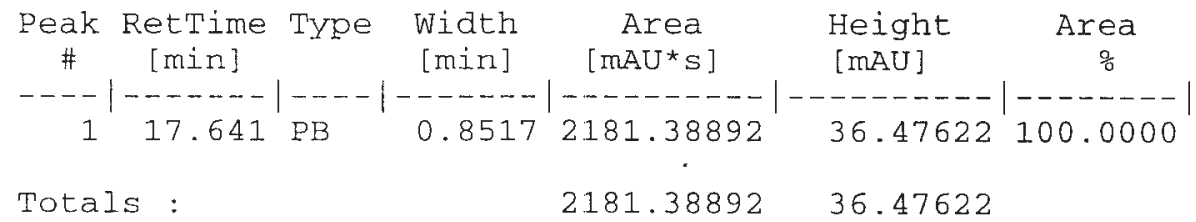

Results obtained with enhanced integrator!

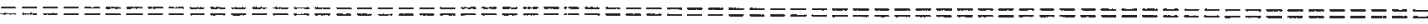

$\star \star \star$ End of Report 


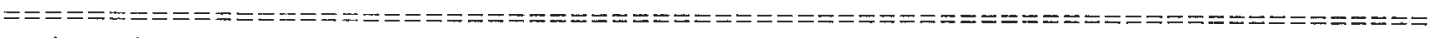

Injection Date : 4/10/2006 10:44:23 AM

Seq. Line : 1

Sample Name

Acq. Operator :

Location : Vial 51

Inj : 1

Inj Volume : $1 \mu 1$

Acq. Method :

Last changed :

Last changed : 4/10/2006 11:15:32 AM

(modified after loading)

$\mathrm{R}, \mathrm{R}$ Whelk-O

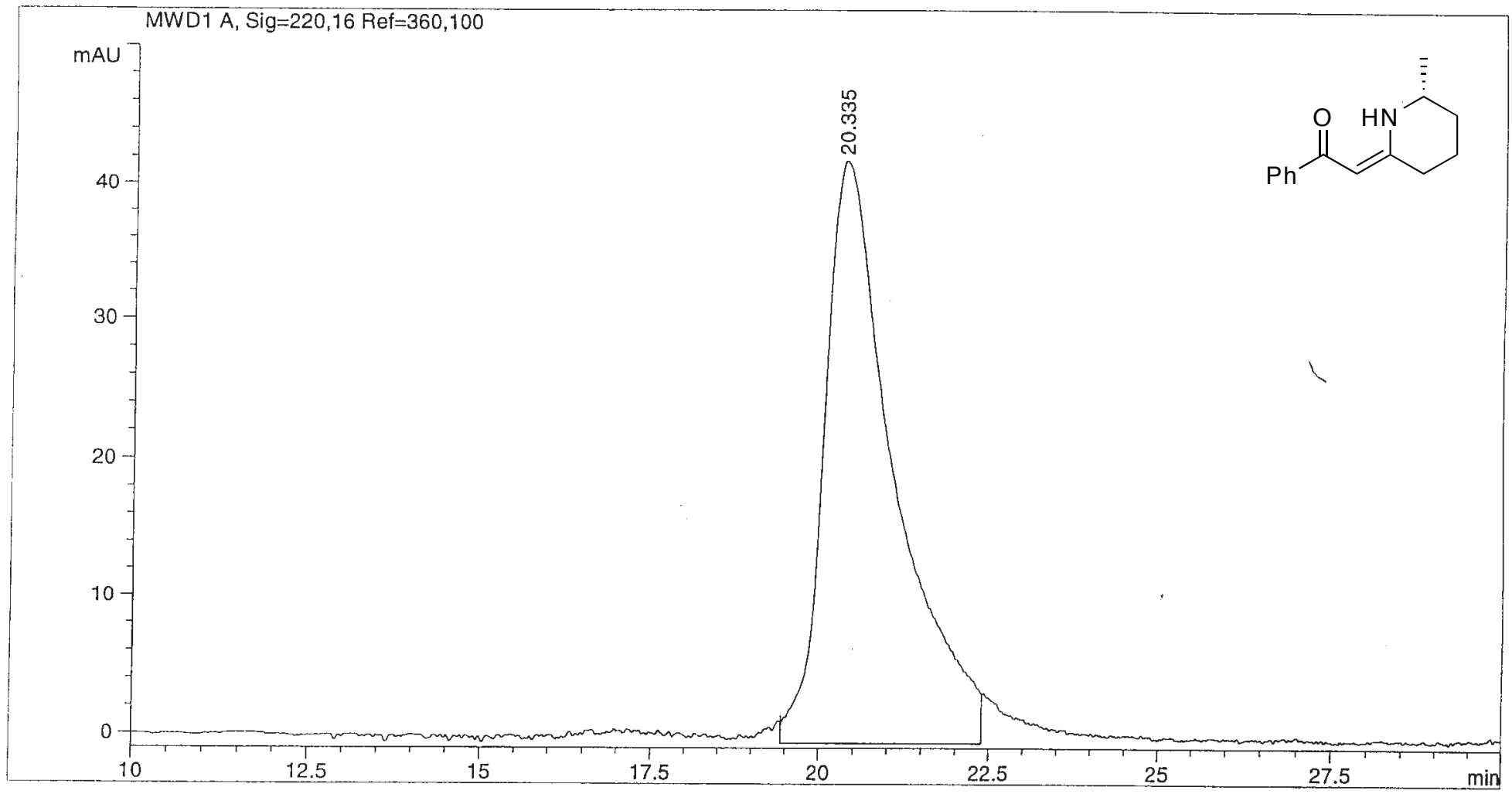

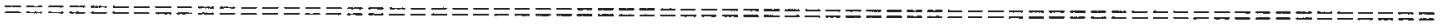

Area Percent Report

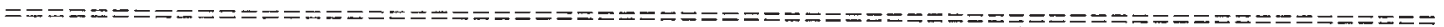

Sorted By : : Signal

Multiplier : : 1.0000

Dilution : 1.0000

Use Multiplier \& Dilution Factor with ISTDS

Signal 1: MWD1 A, Sig=220,16 Ref $=360,100$

Peak RetTime Type width Area Height Area

\begin{tabular}{c|c|c|c|c|}
$\#$ & {$[\min ]$} & {$[\mathrm{min}]$} & {$\left[\mathrm{mAU}{ }^{*} \mathrm{~S}\right]$} & [mAU]
\end{tabular}

120.335 VV $\quad 0.86303016 .49365 \quad 42.44931100 .0000$

Totals : $\quad 3016.49365 \quad 42.44931$

Results obtained with enhanced integrator!

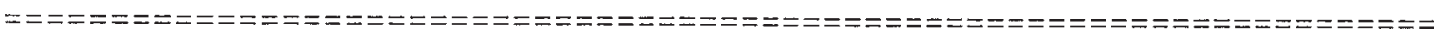

*** End of Report. *** 


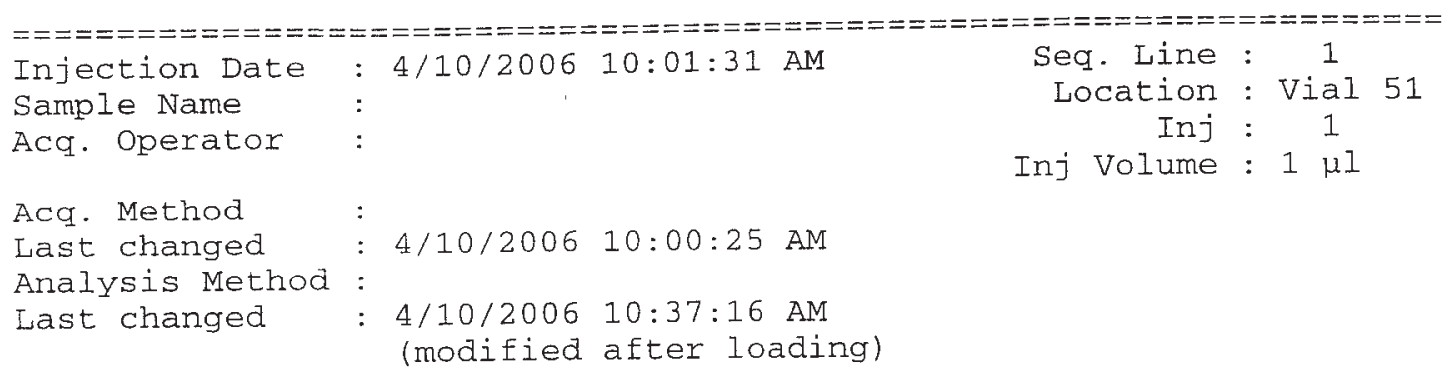

R, R Whelk-O

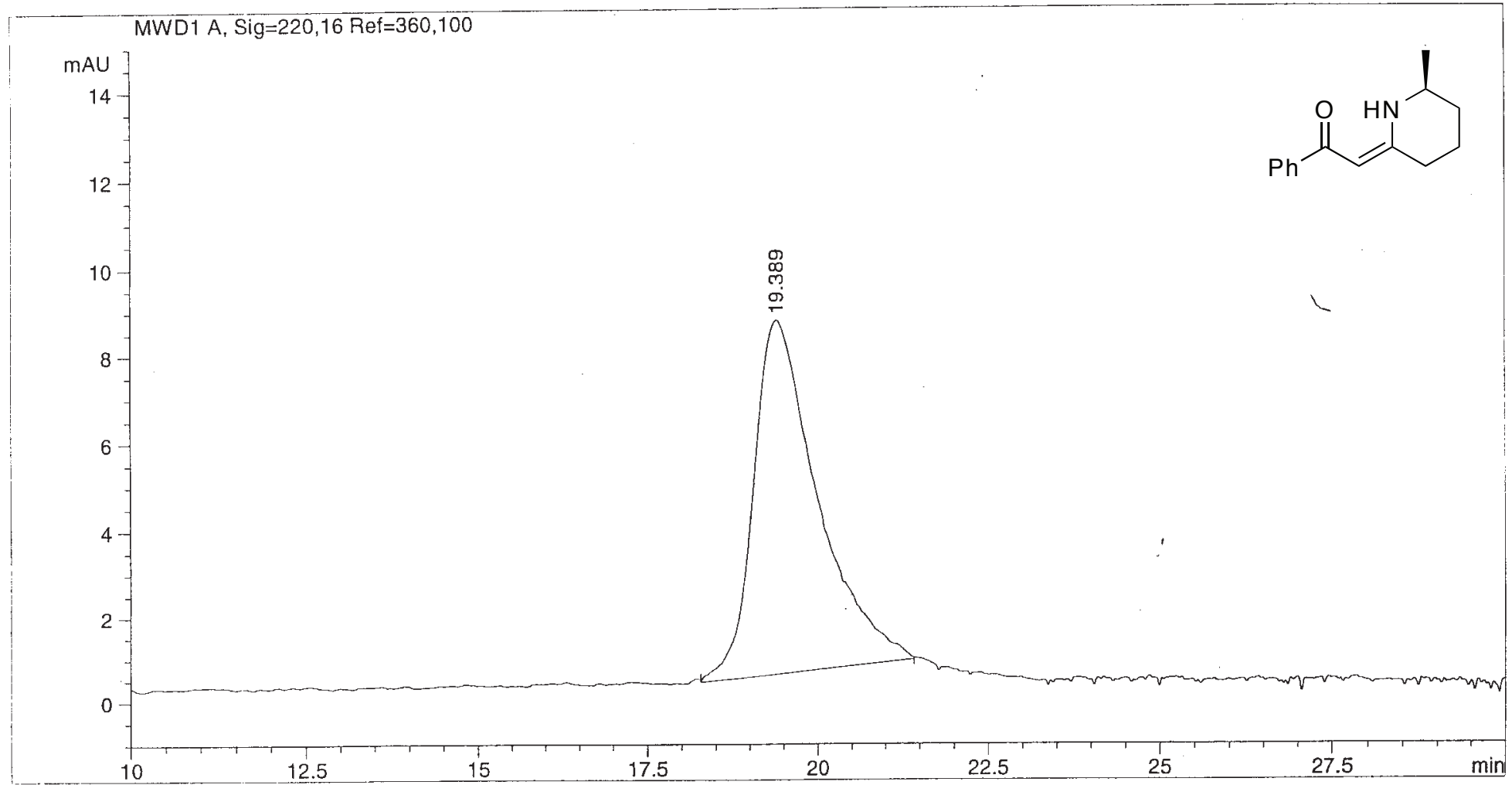

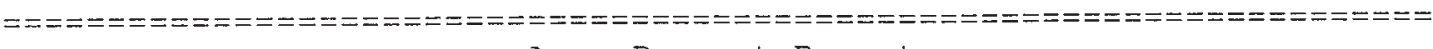
Area Percent Report

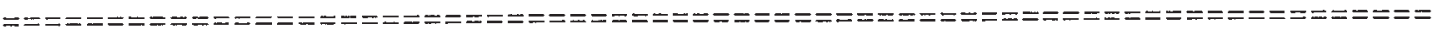

$\begin{array}{lll}\text { Sorted By } & : & \text { Signal } \\ \text { Multiplier } & : & 1.0000 \\ \text { Dilution } & : & 1.0000\end{array}$

Use Multiplier \& Dilution Factor with ISTDS

Signal 1: MWD1 A, Sig=220,16 Ref $=360,100$

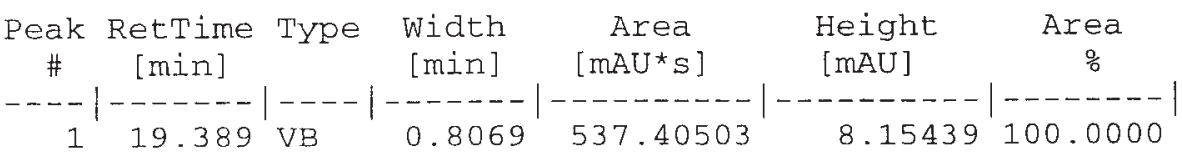

Totals :

$537.40503 \quad 8.15439$

Results obtained with enhanced integrator!

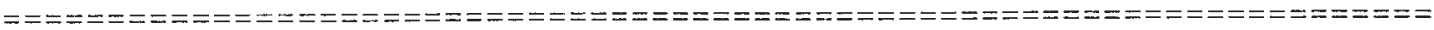

*** End of Report *** 


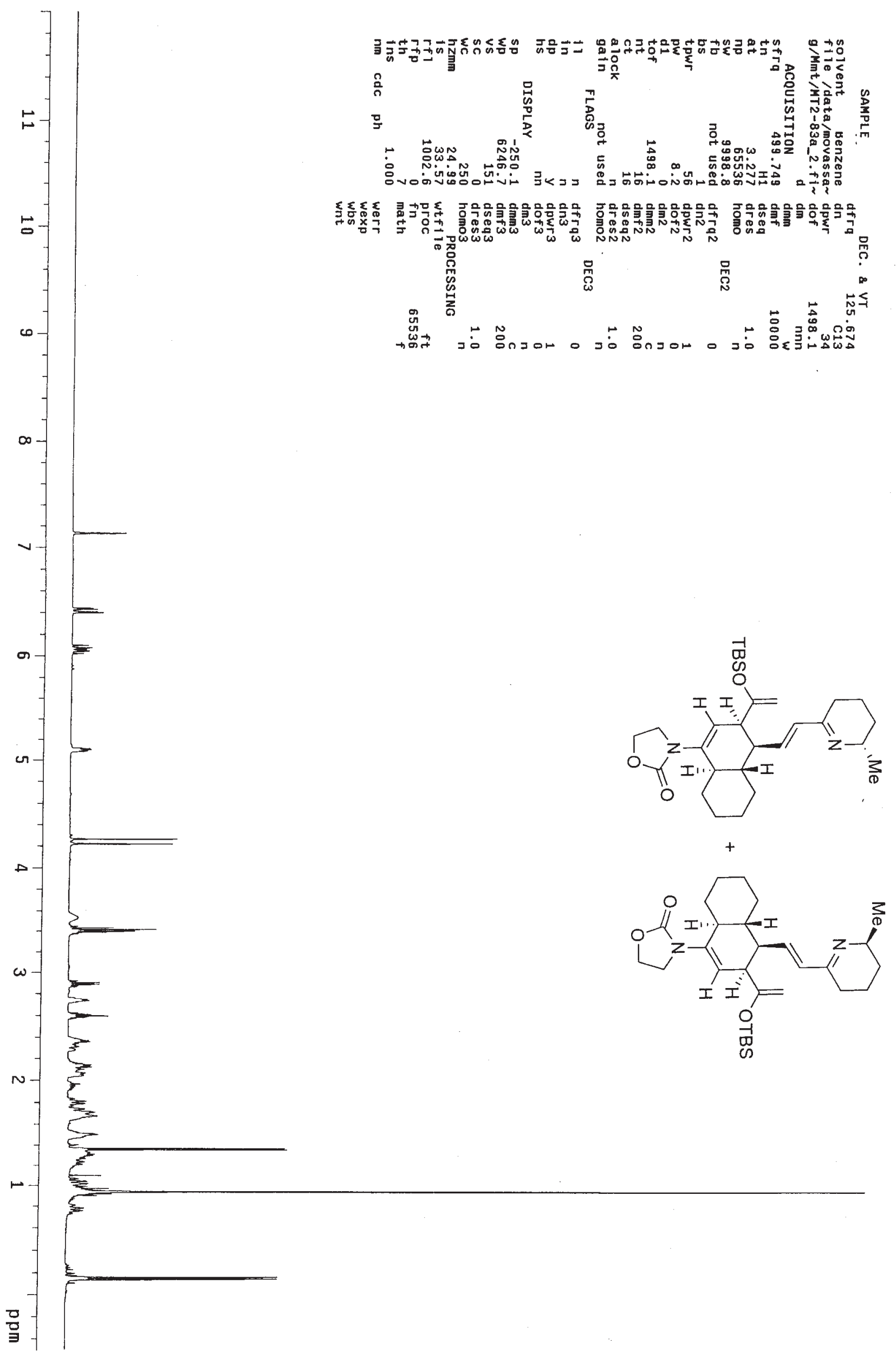




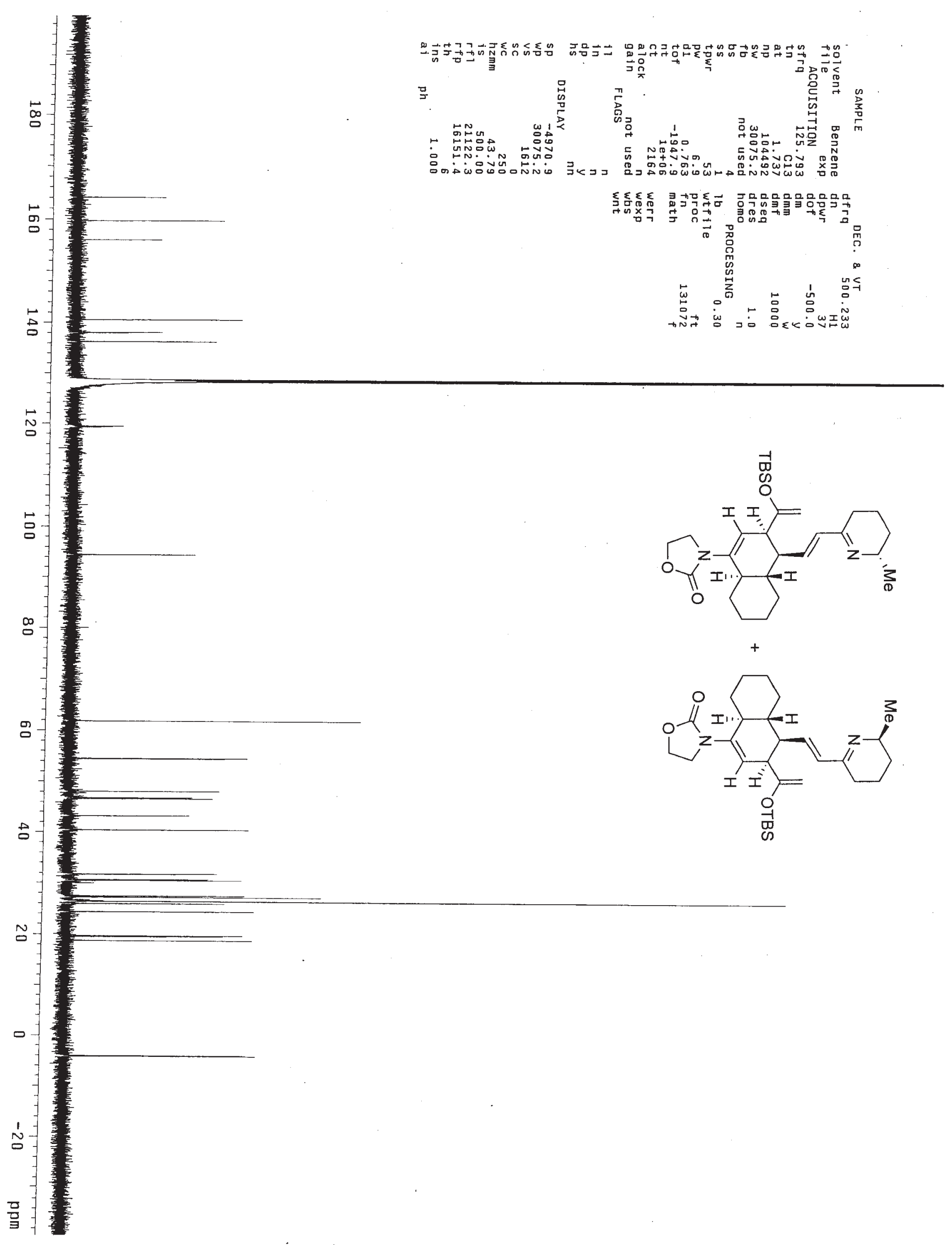




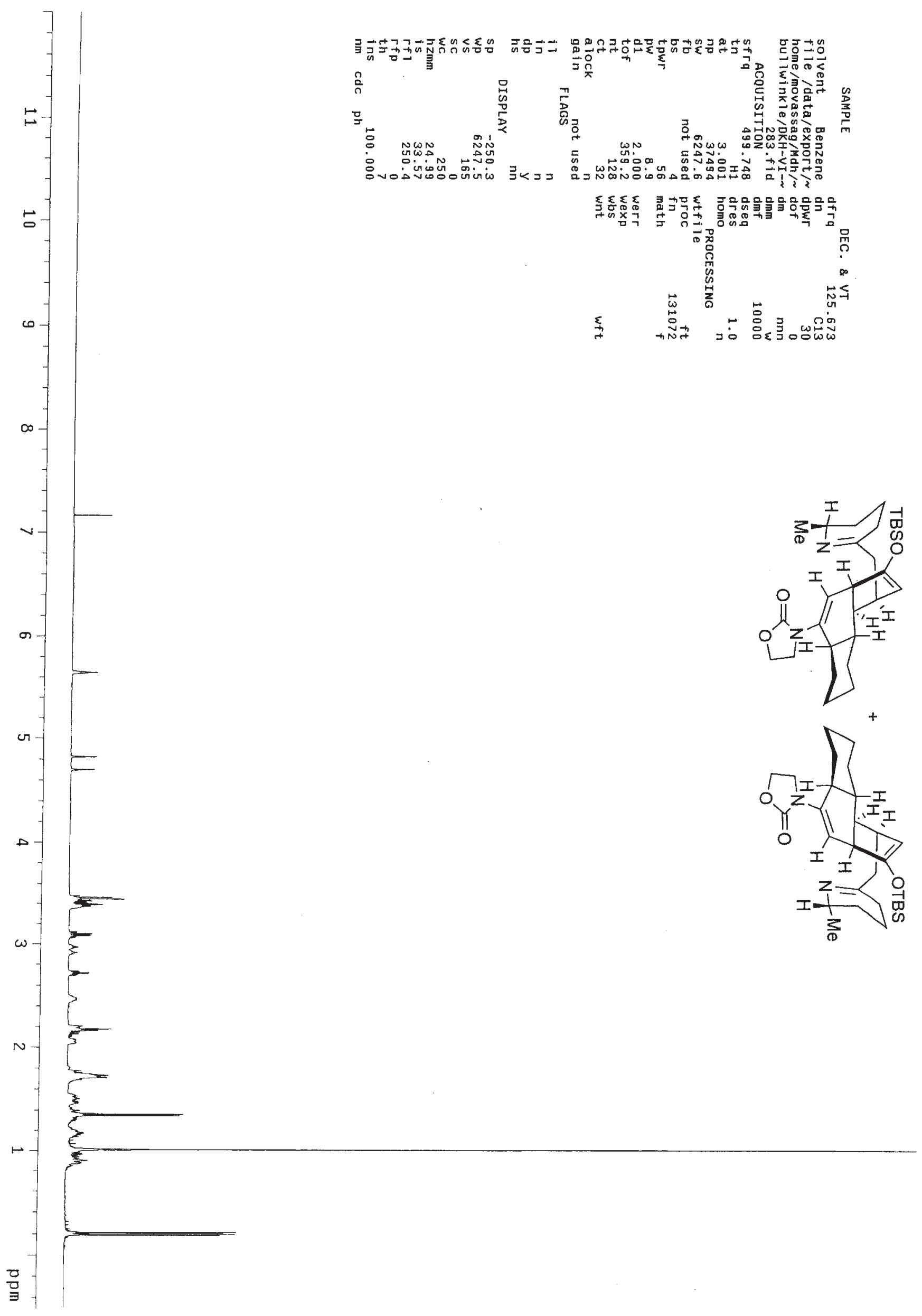




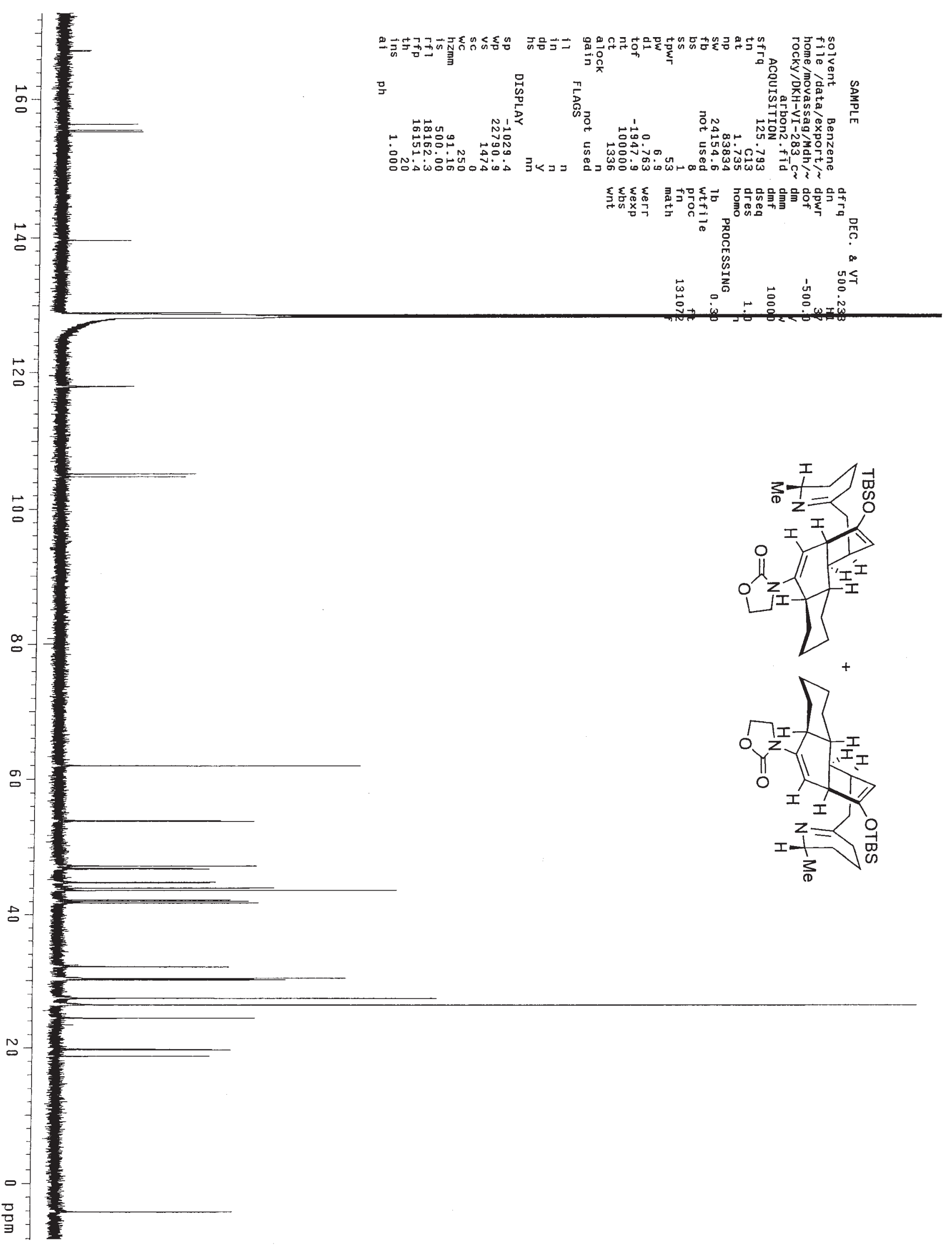




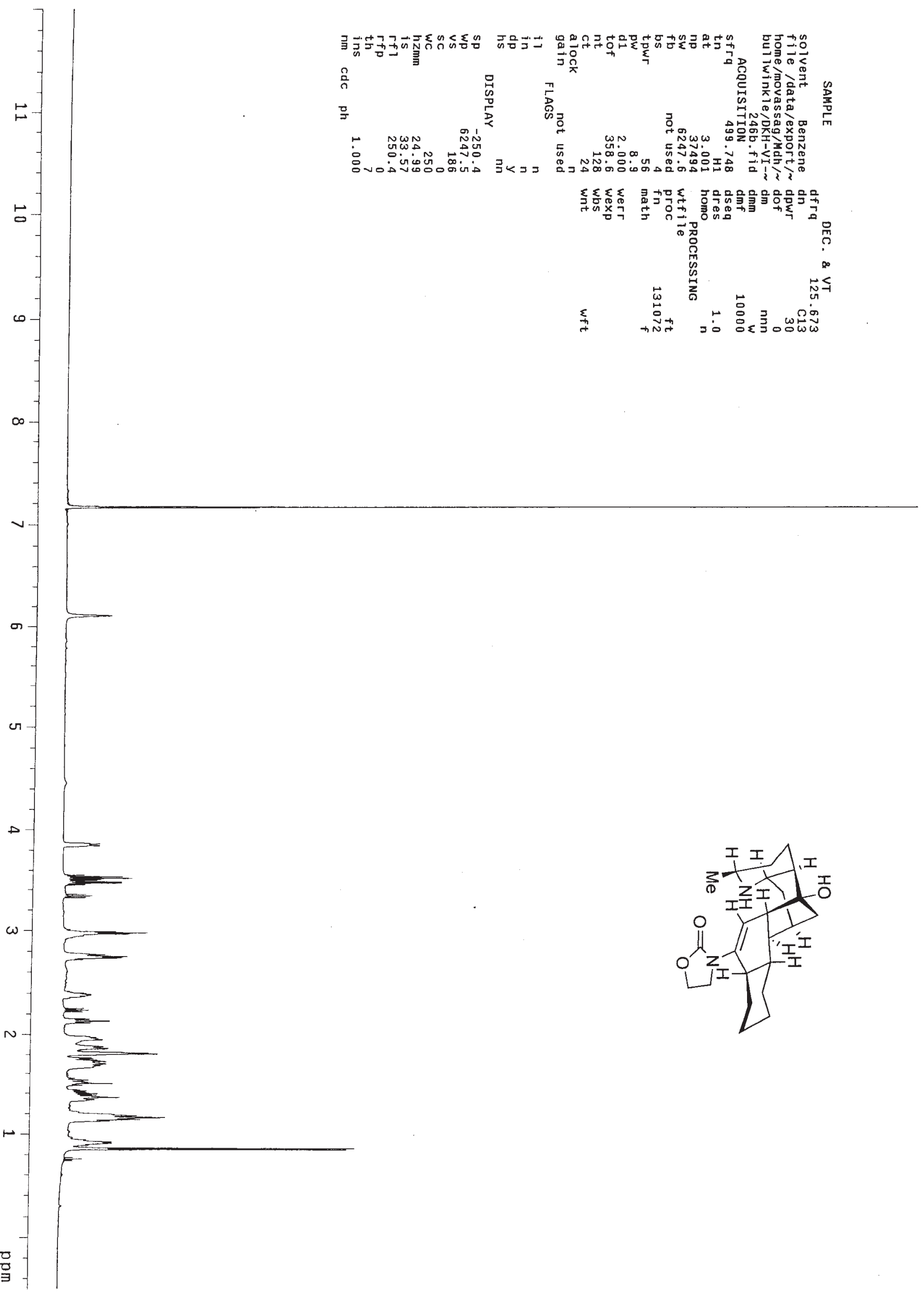




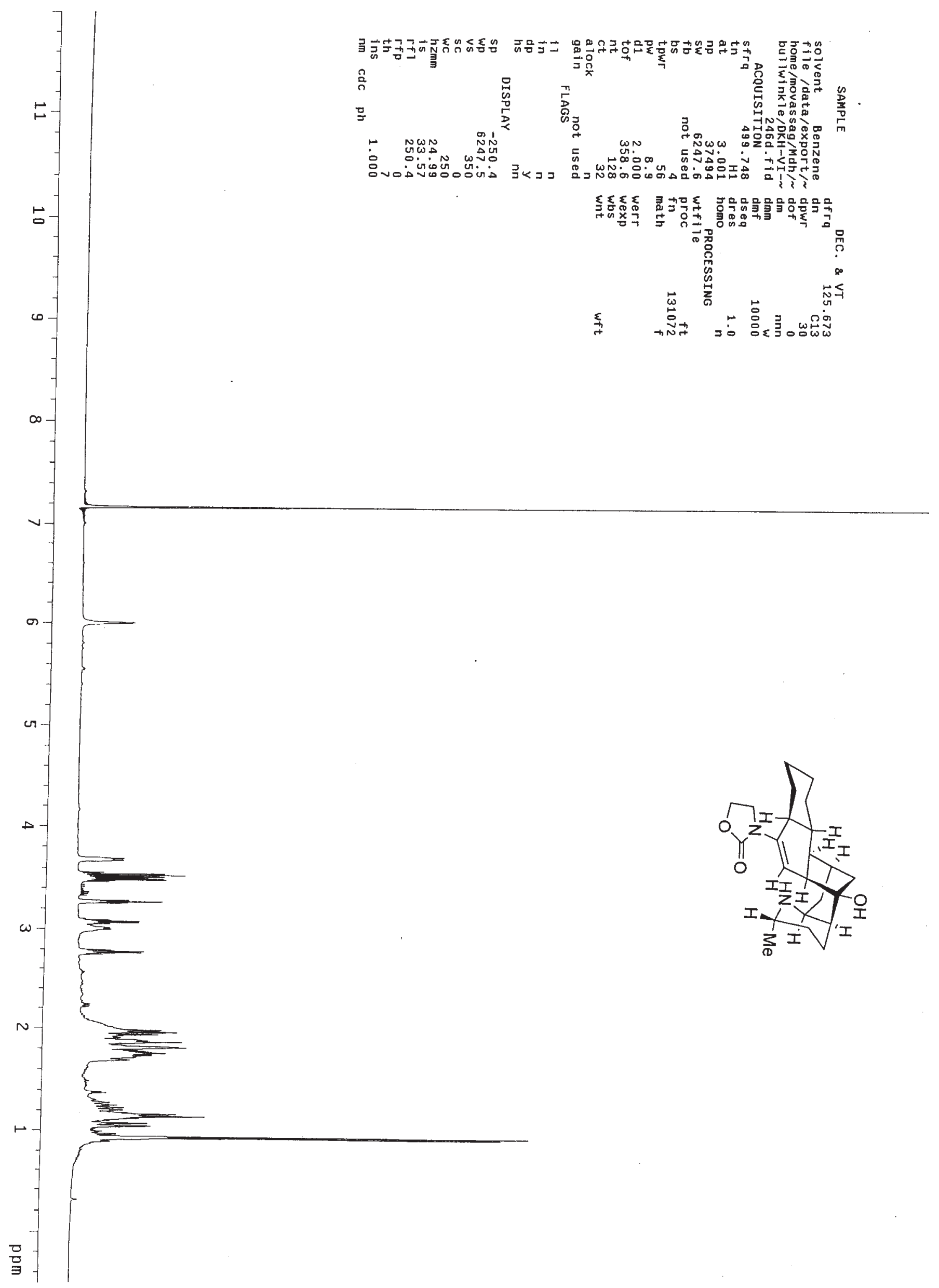




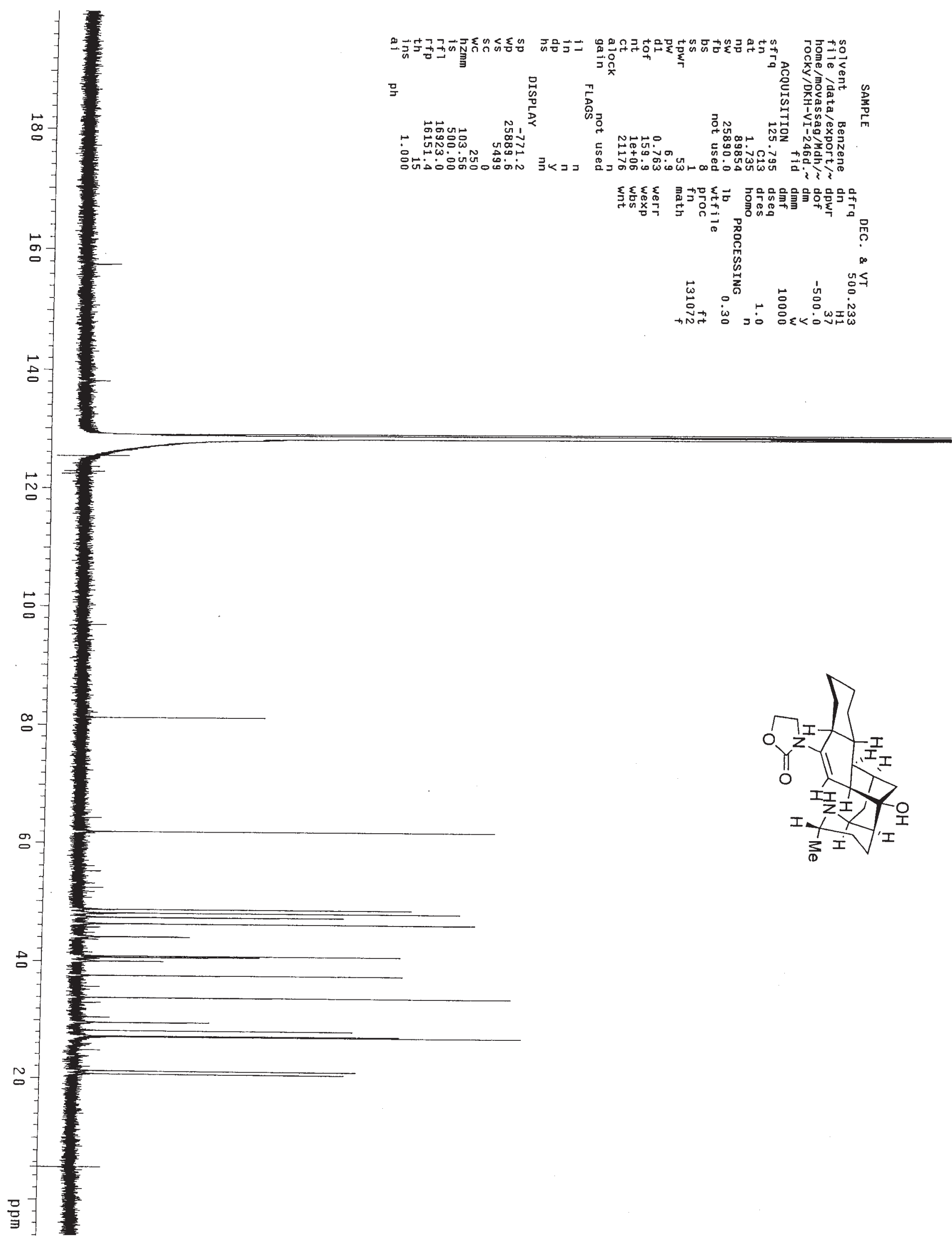




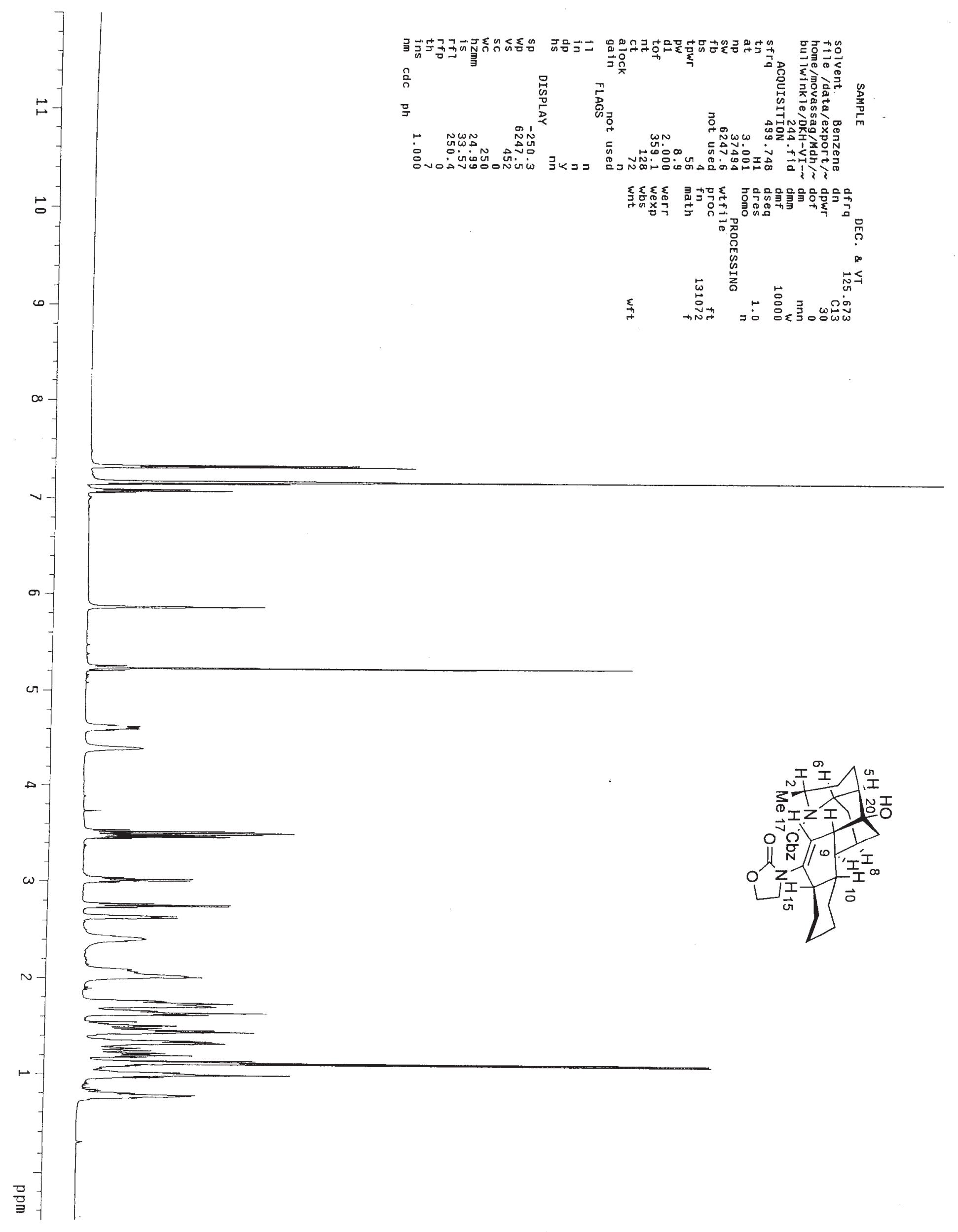




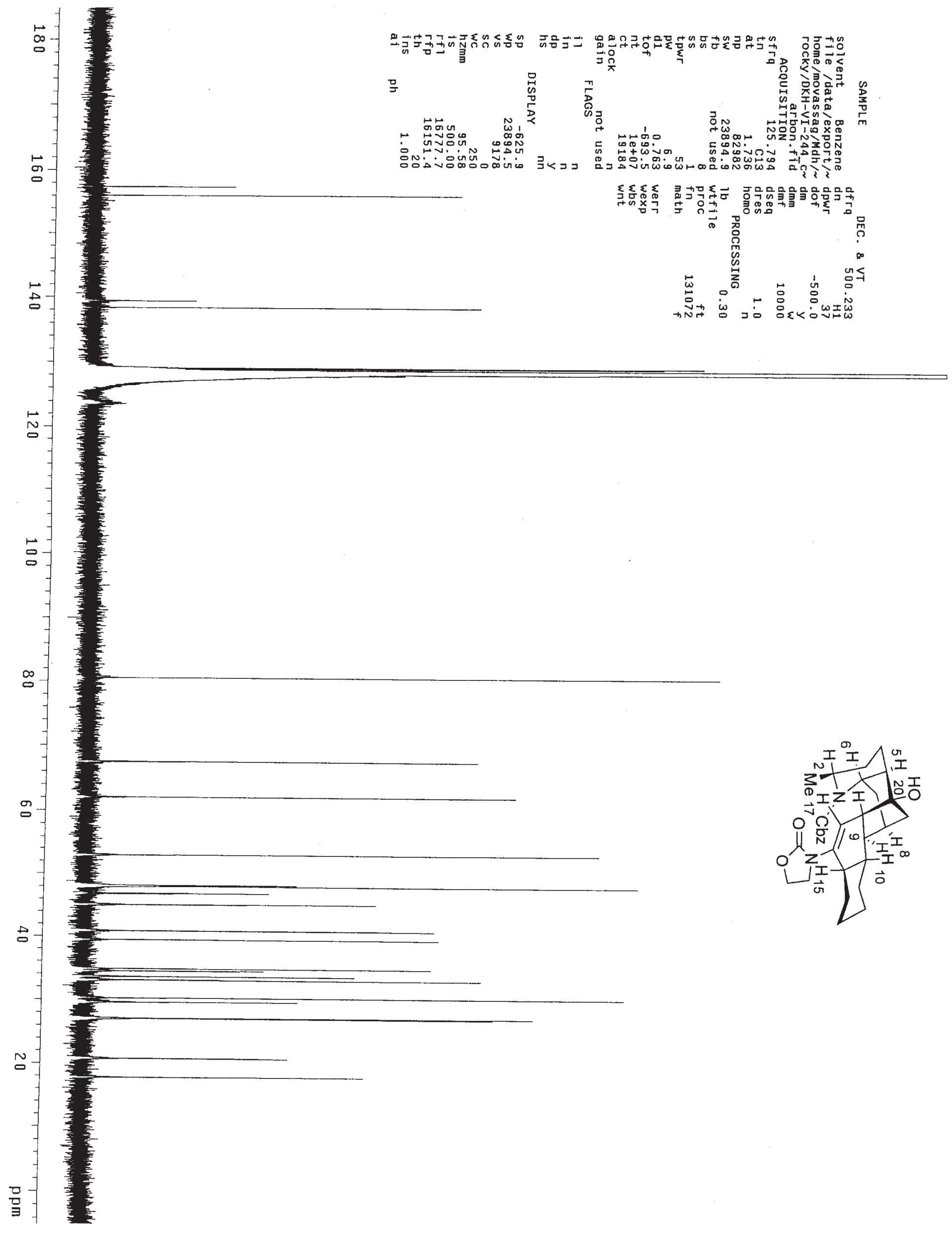




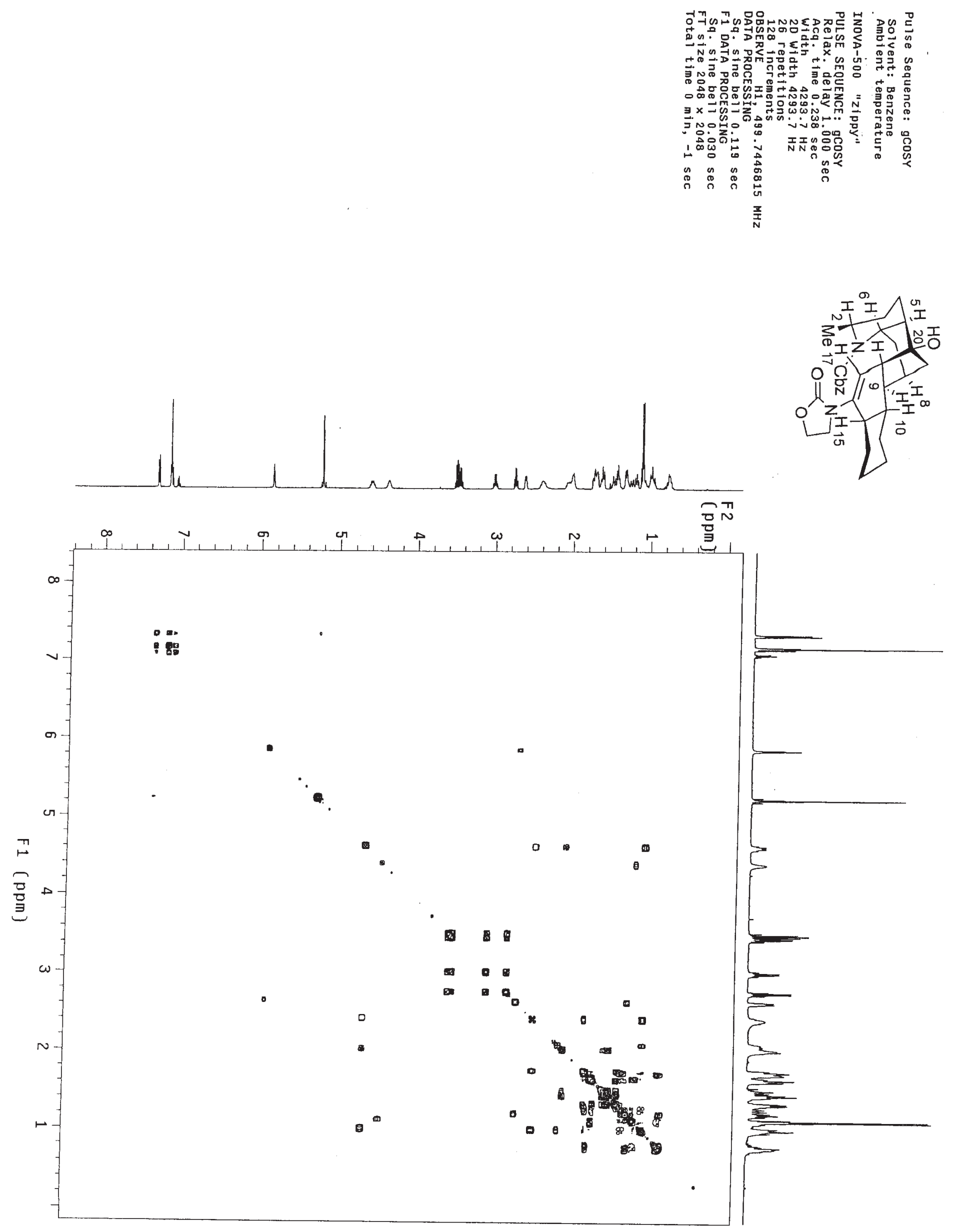




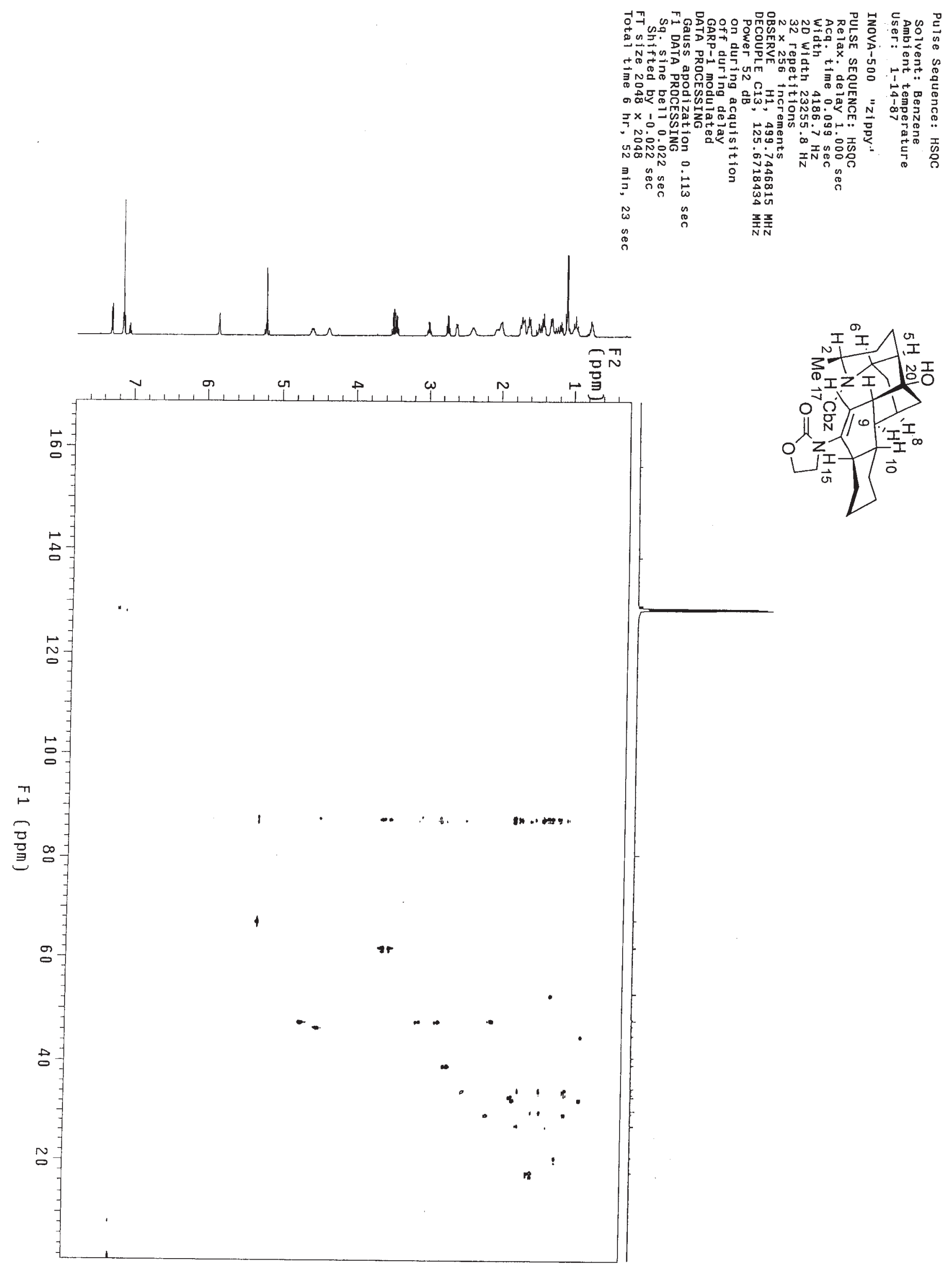




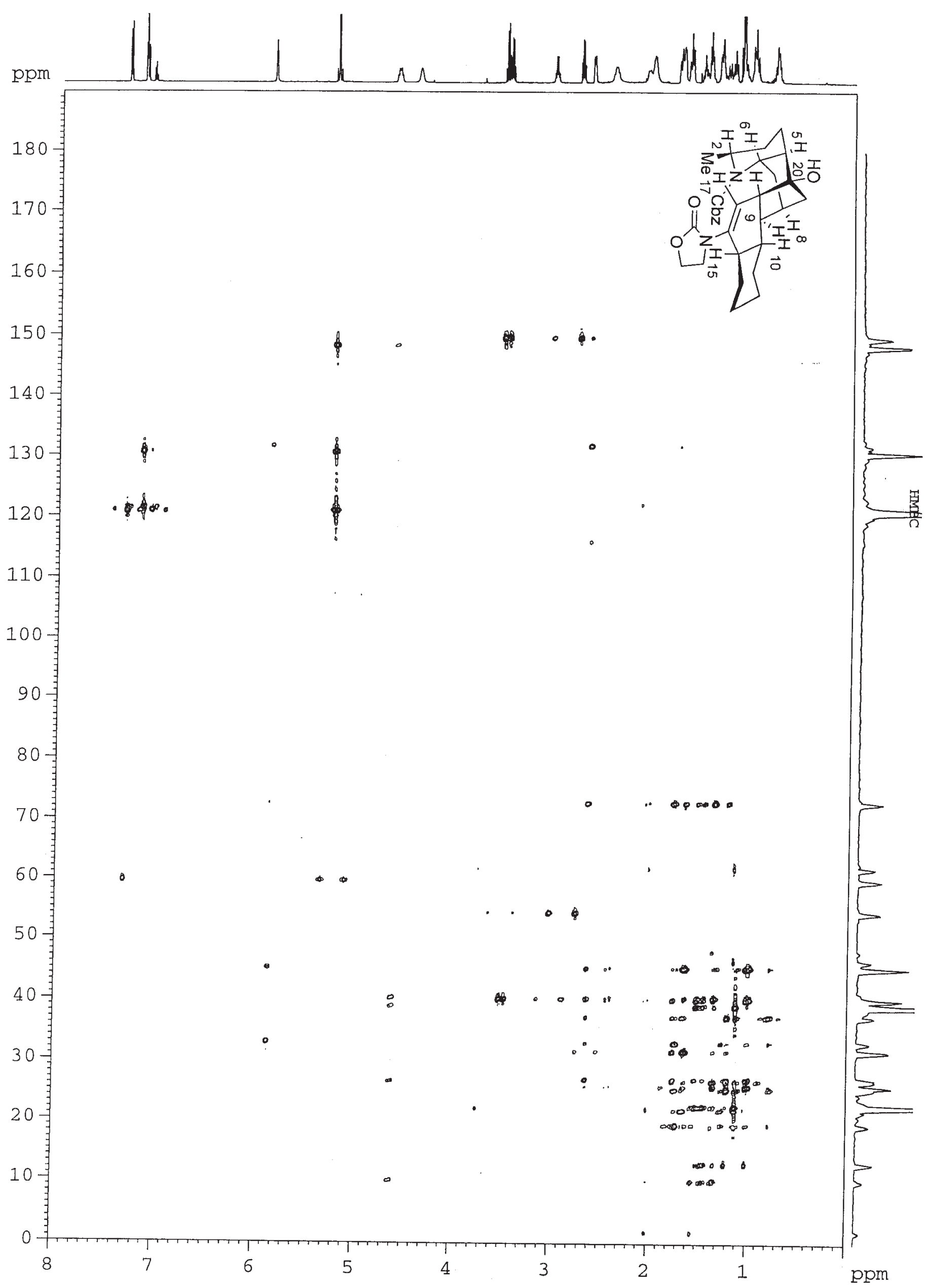




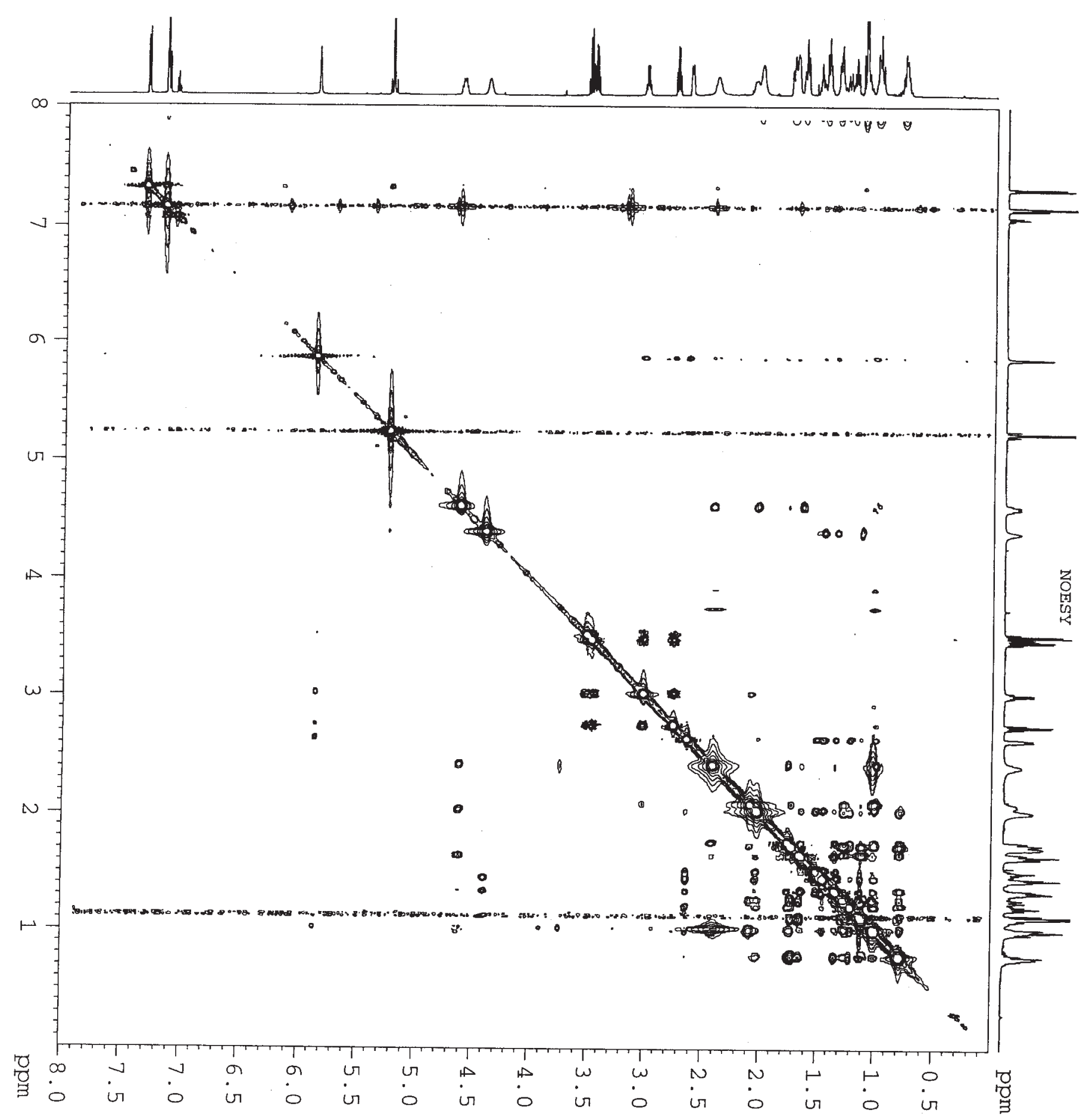

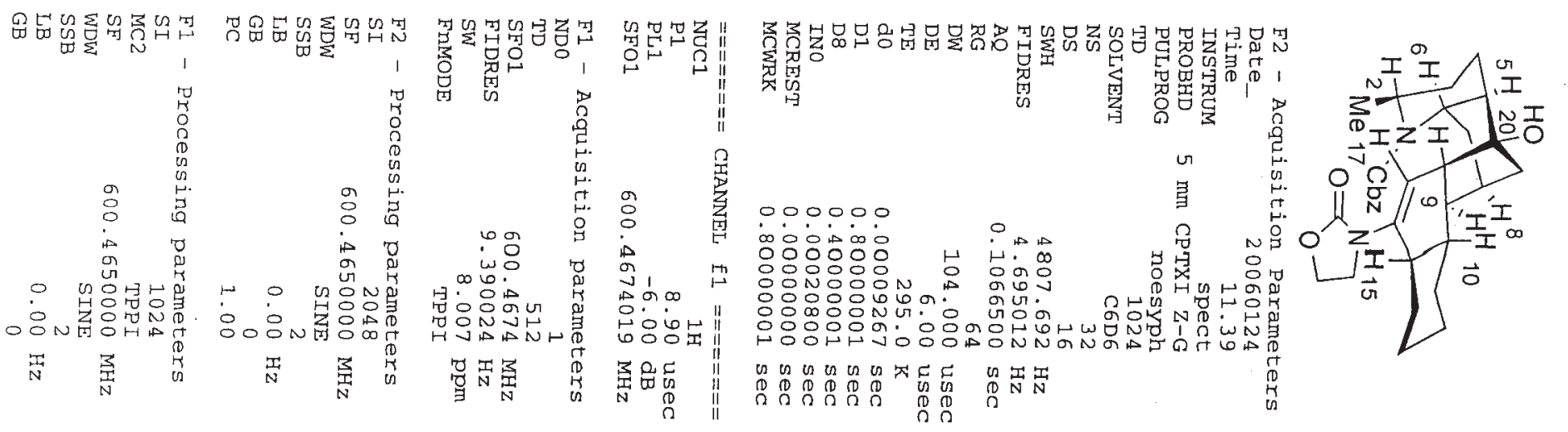




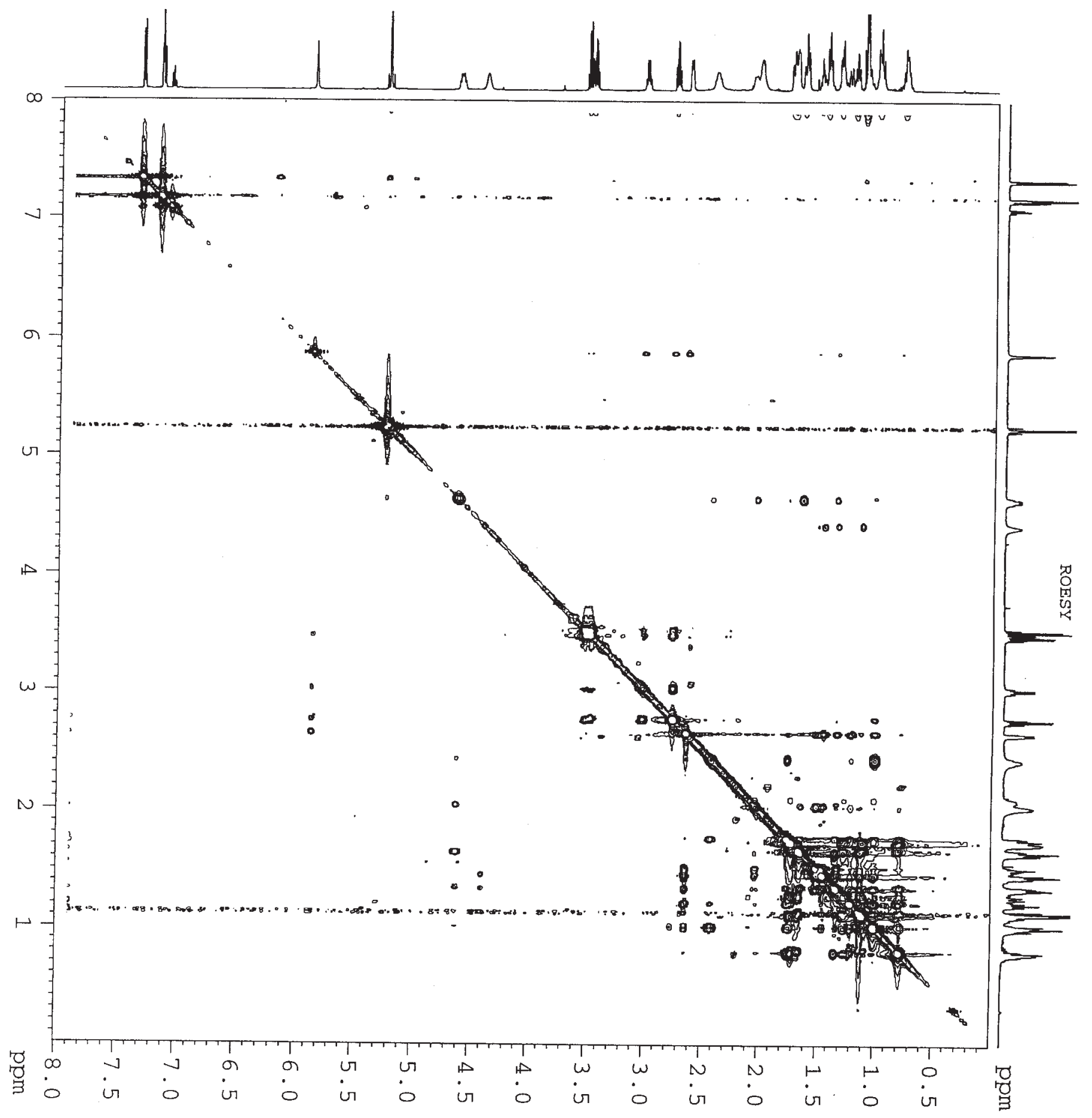

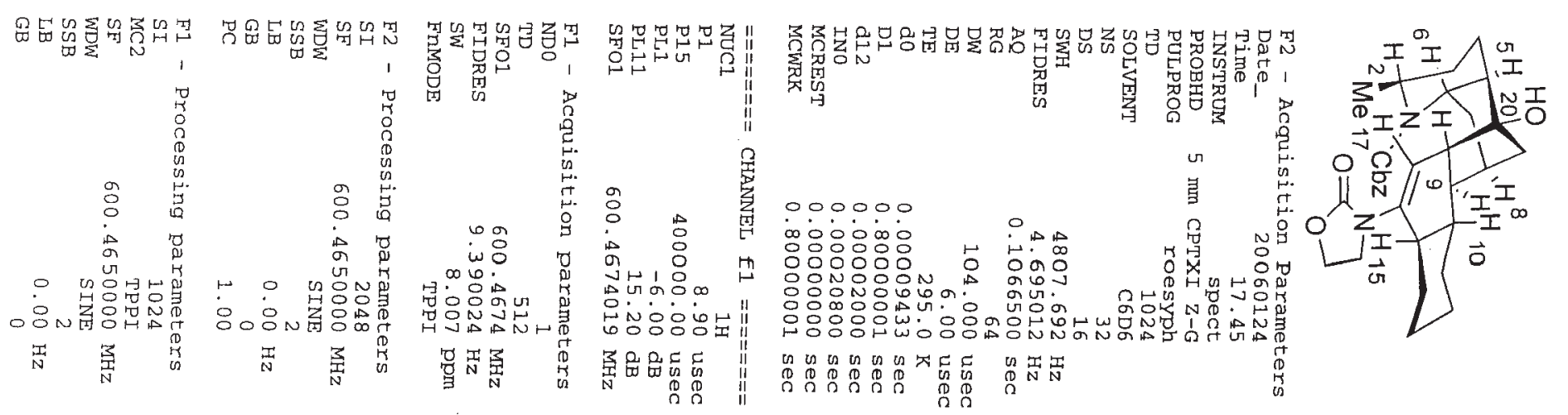




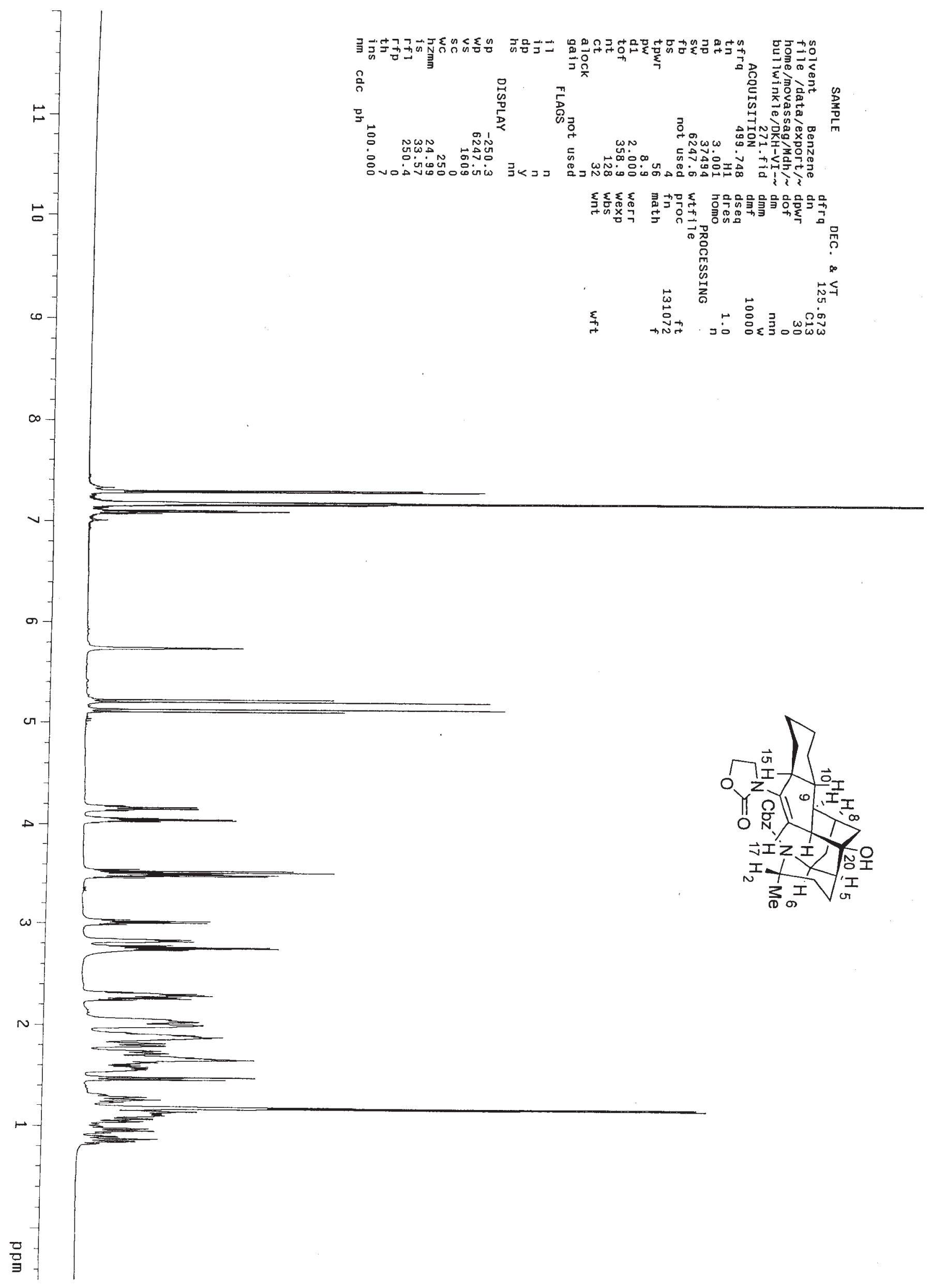




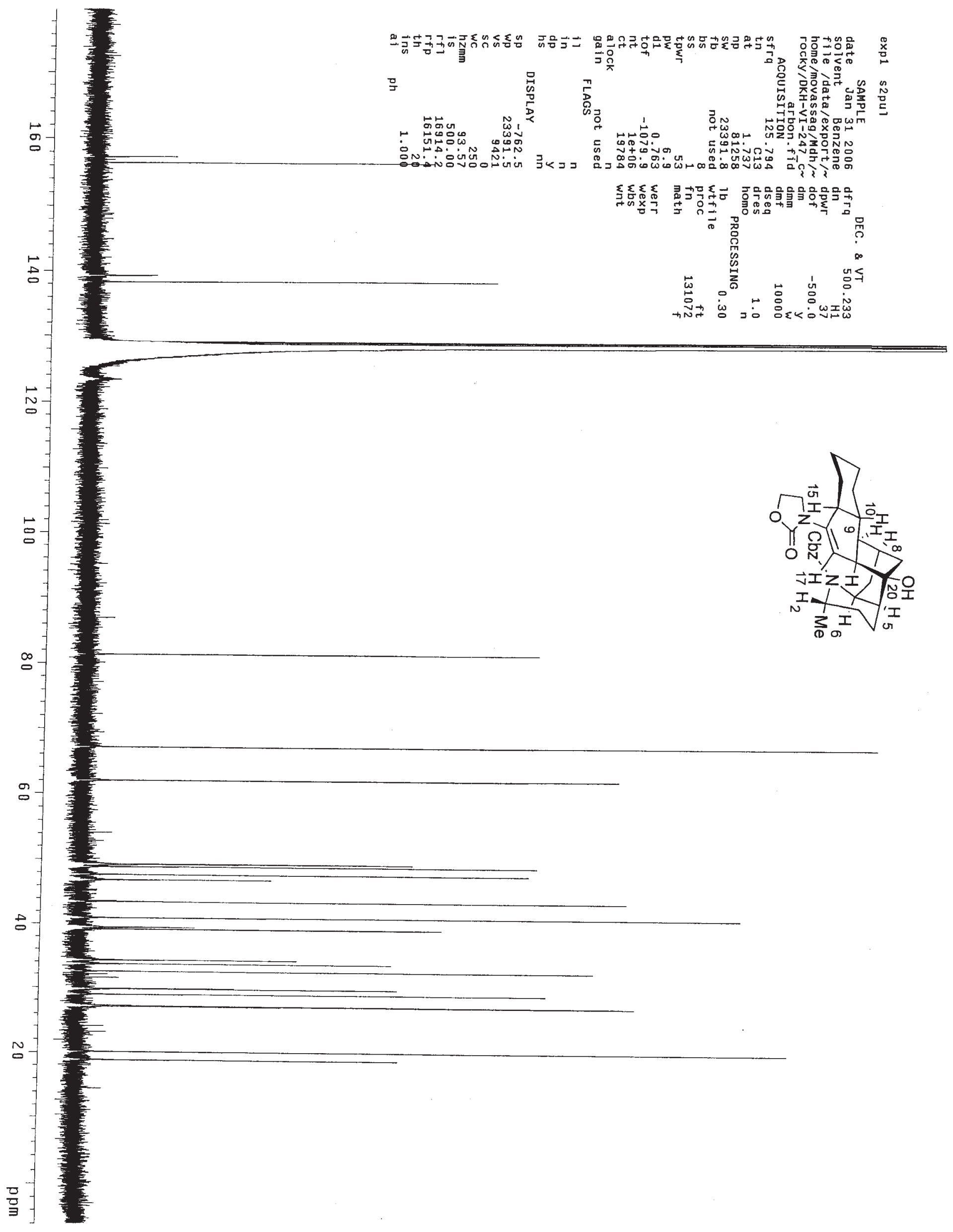




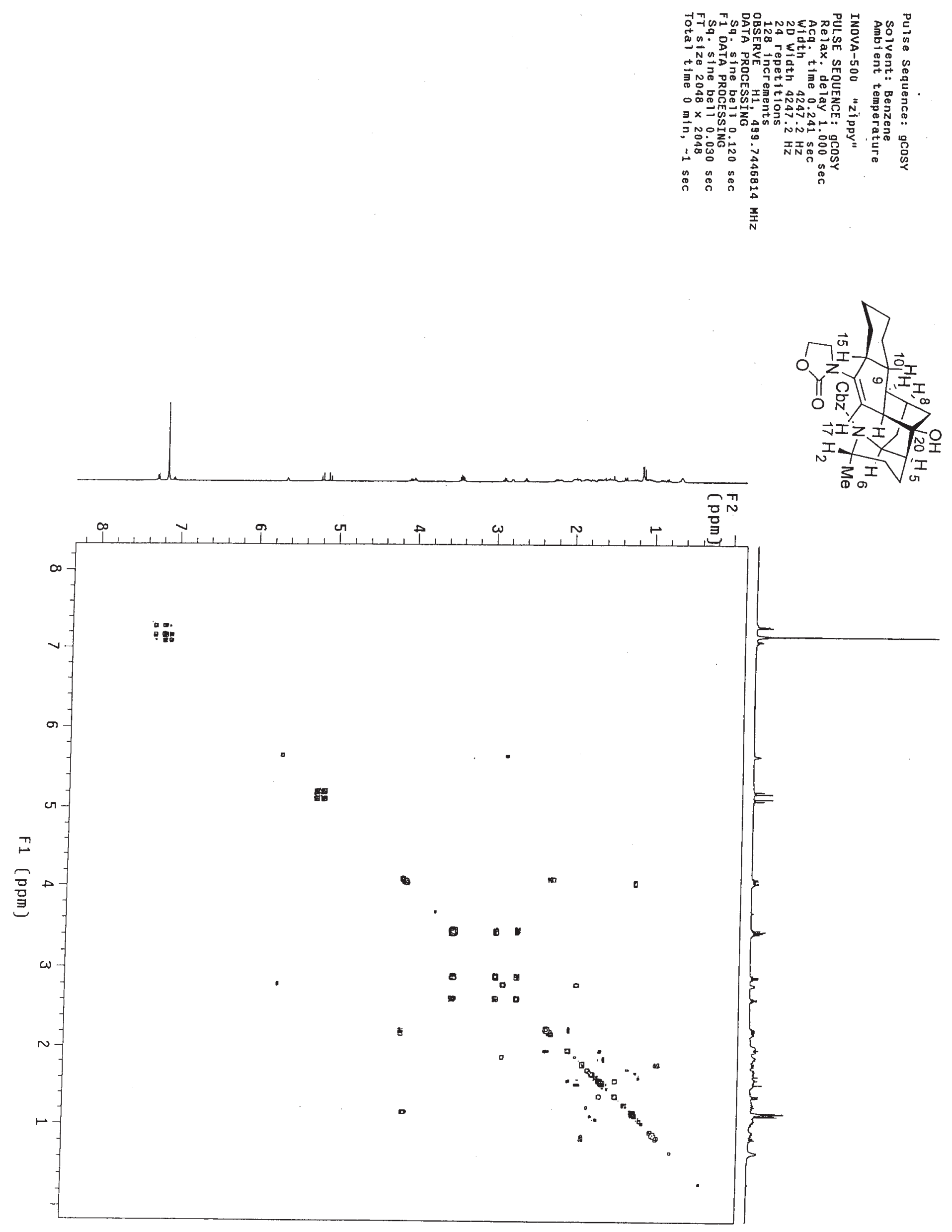




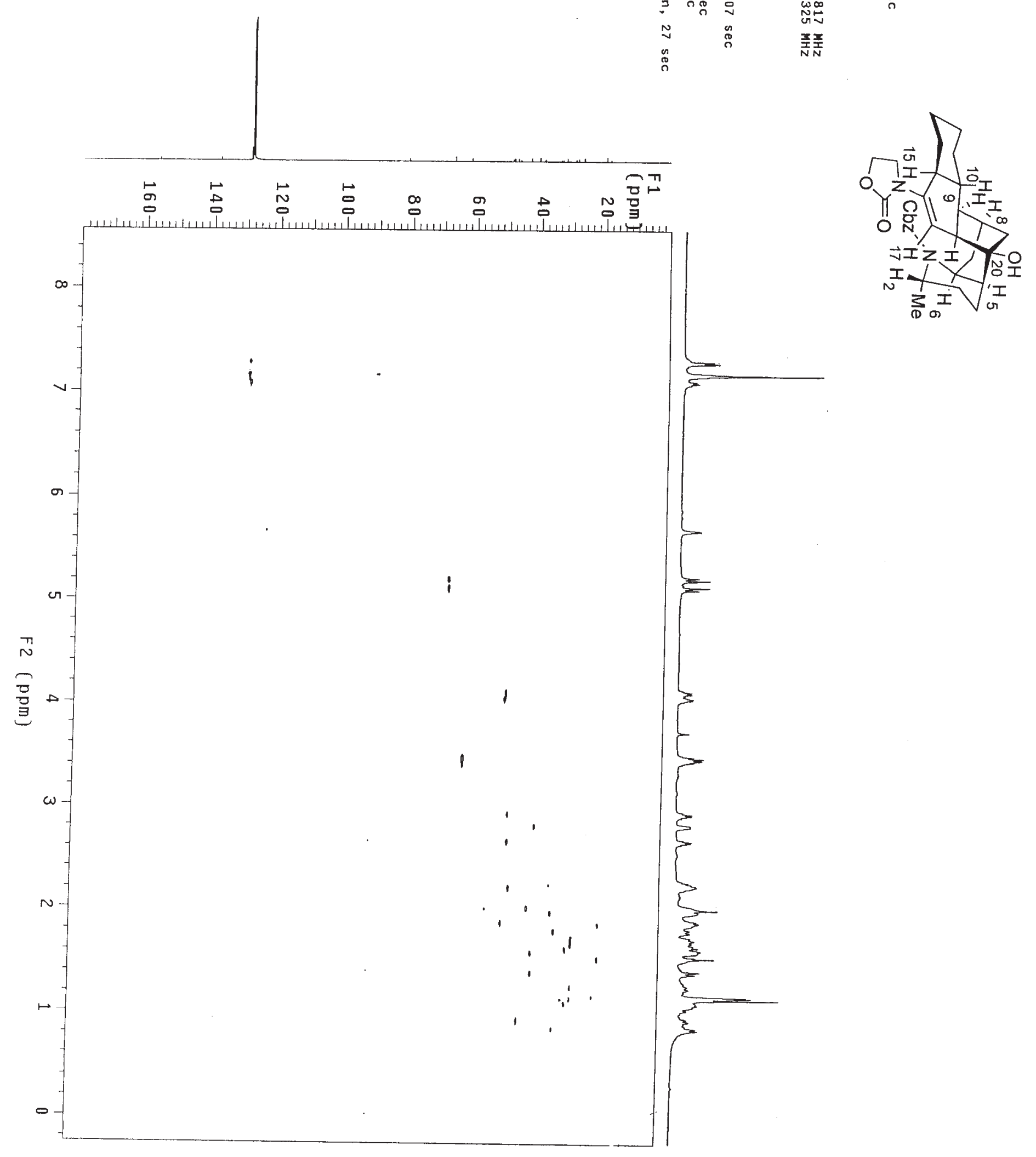




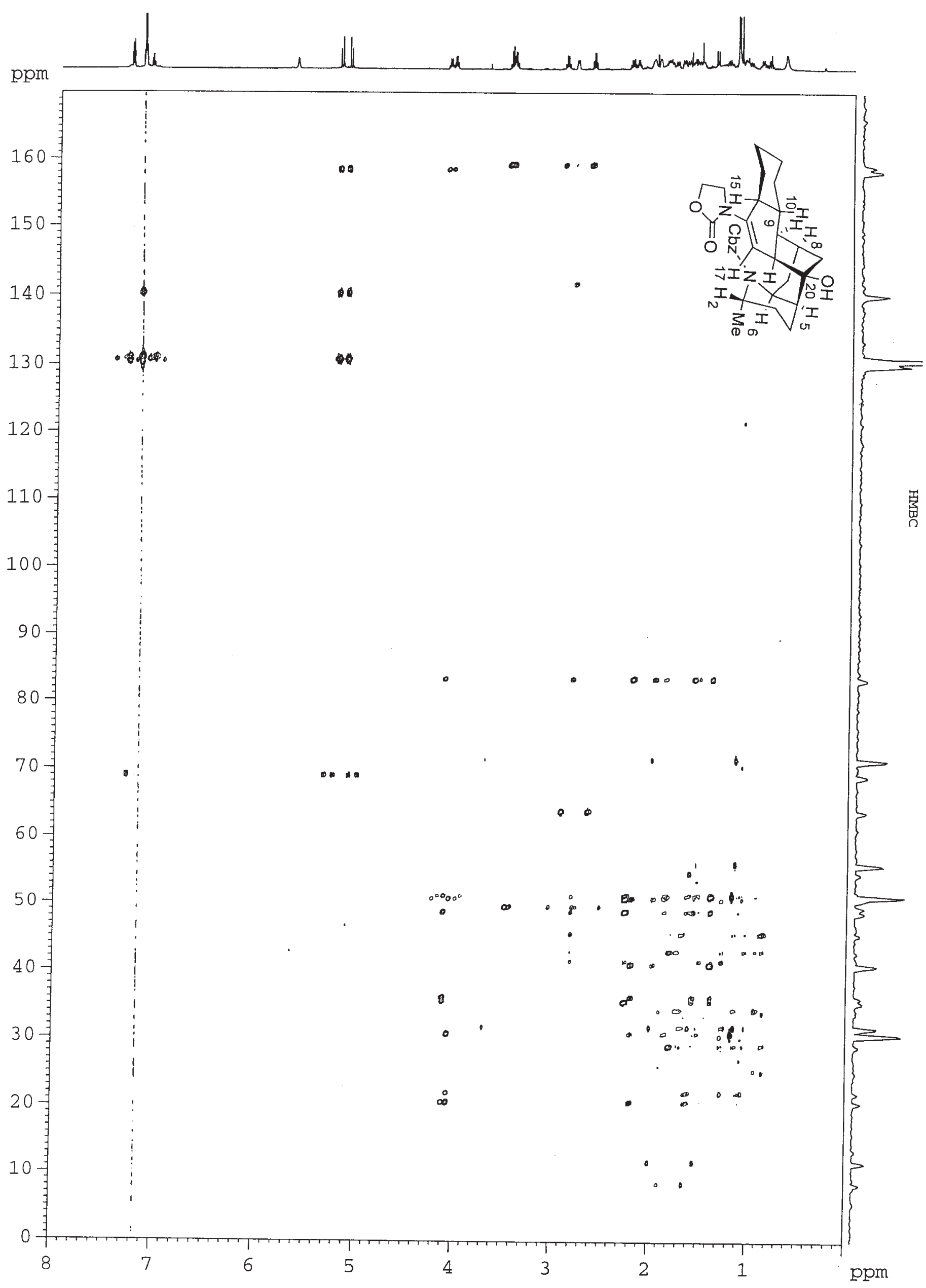



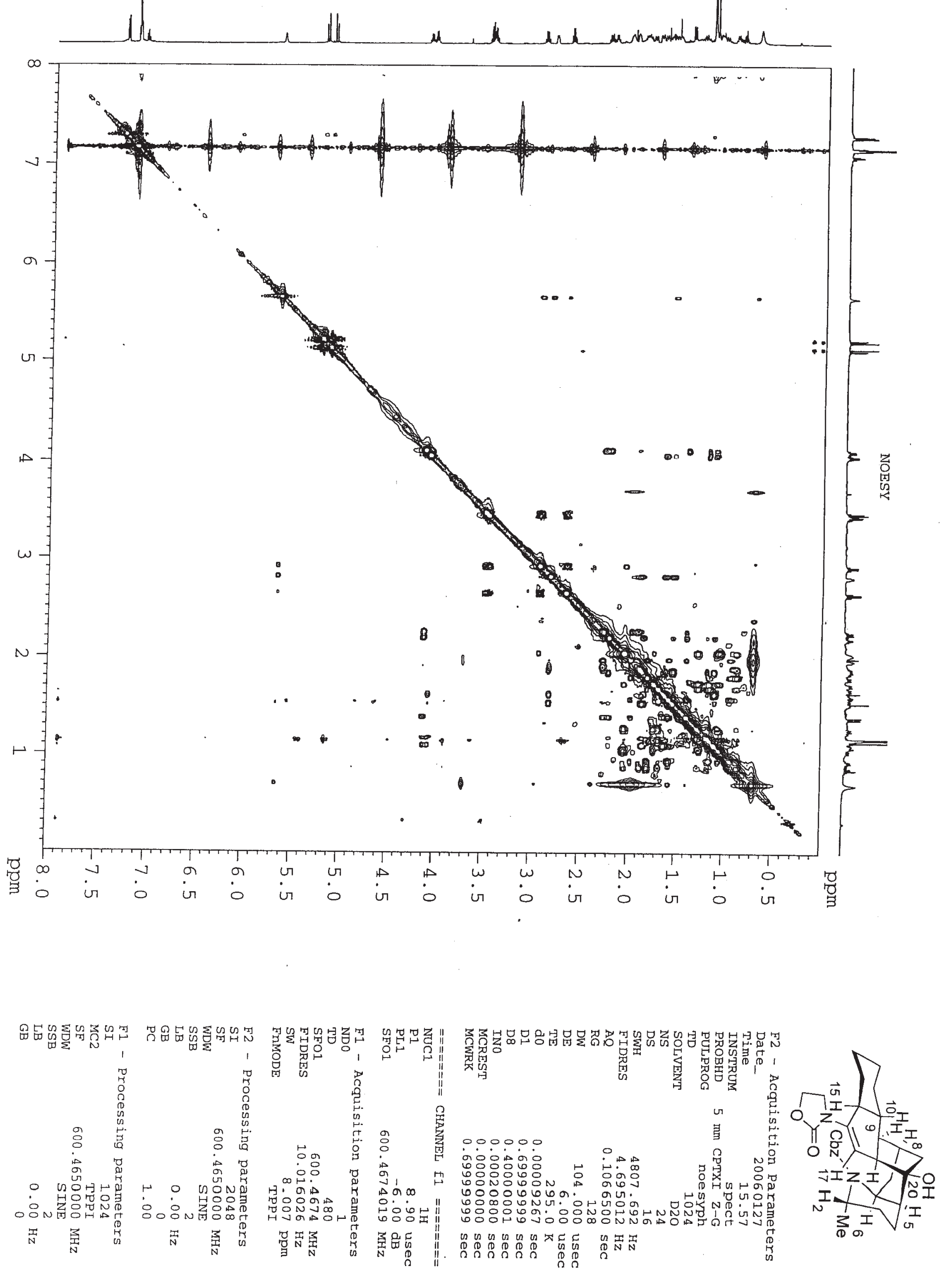

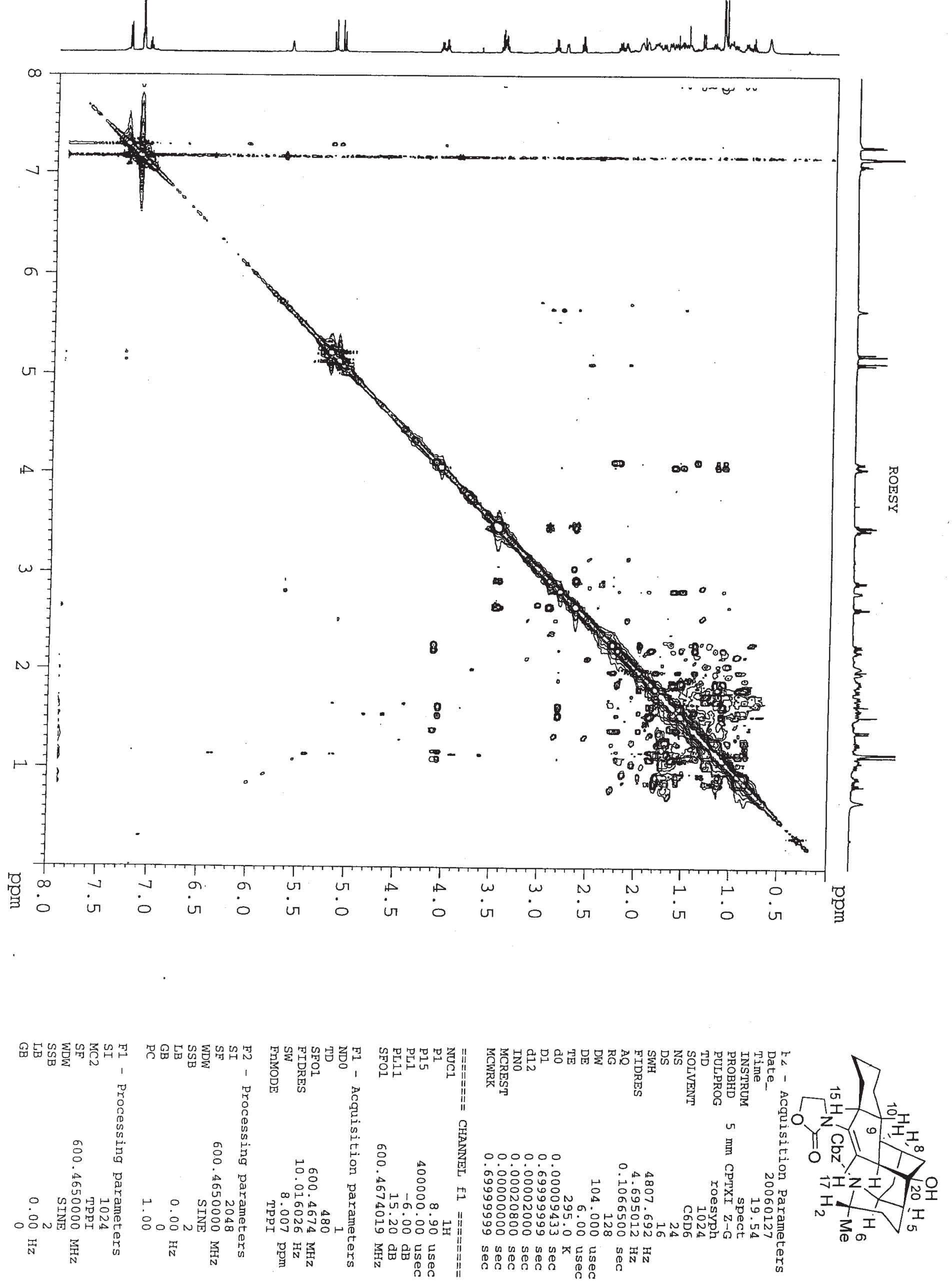


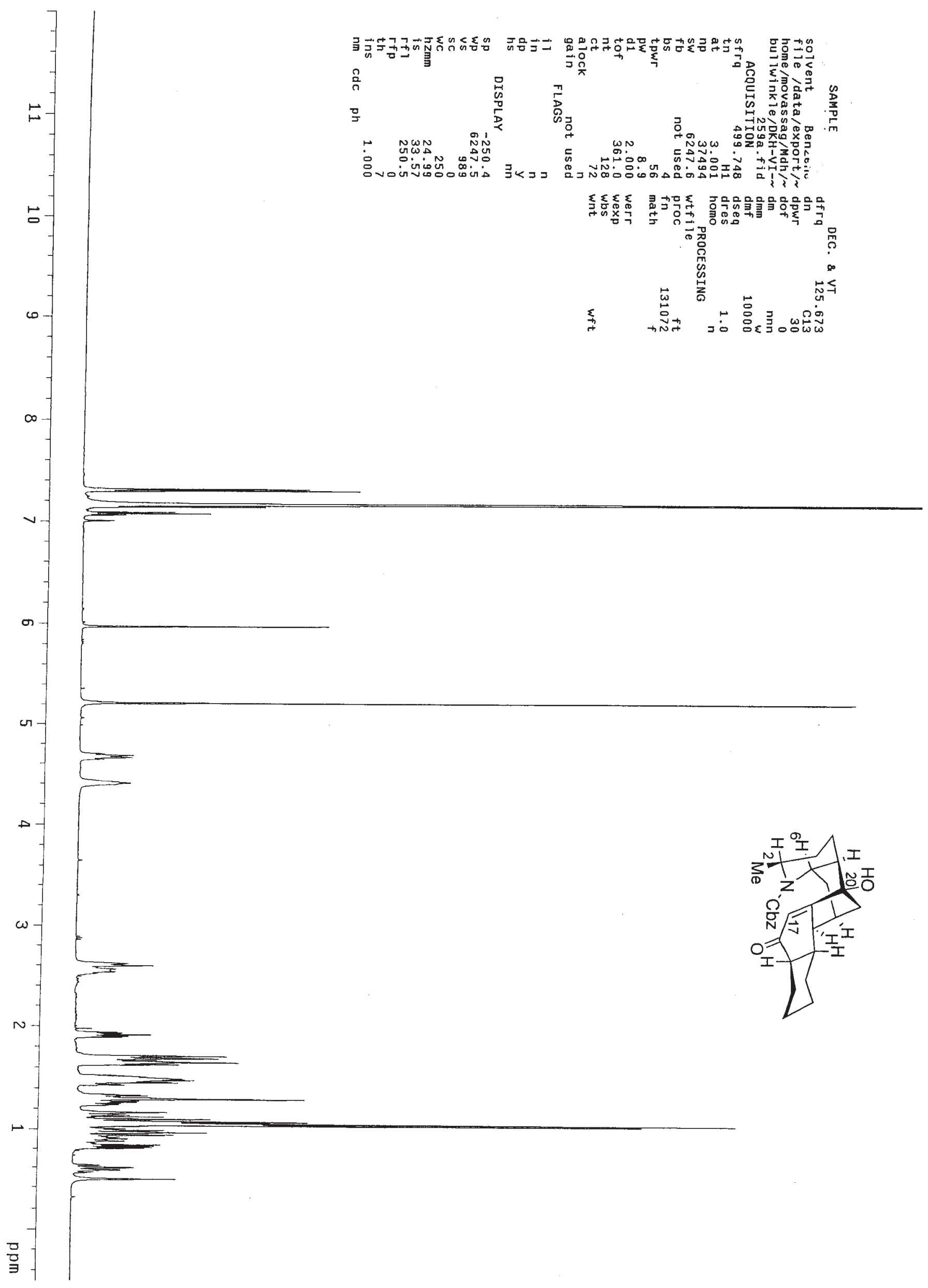




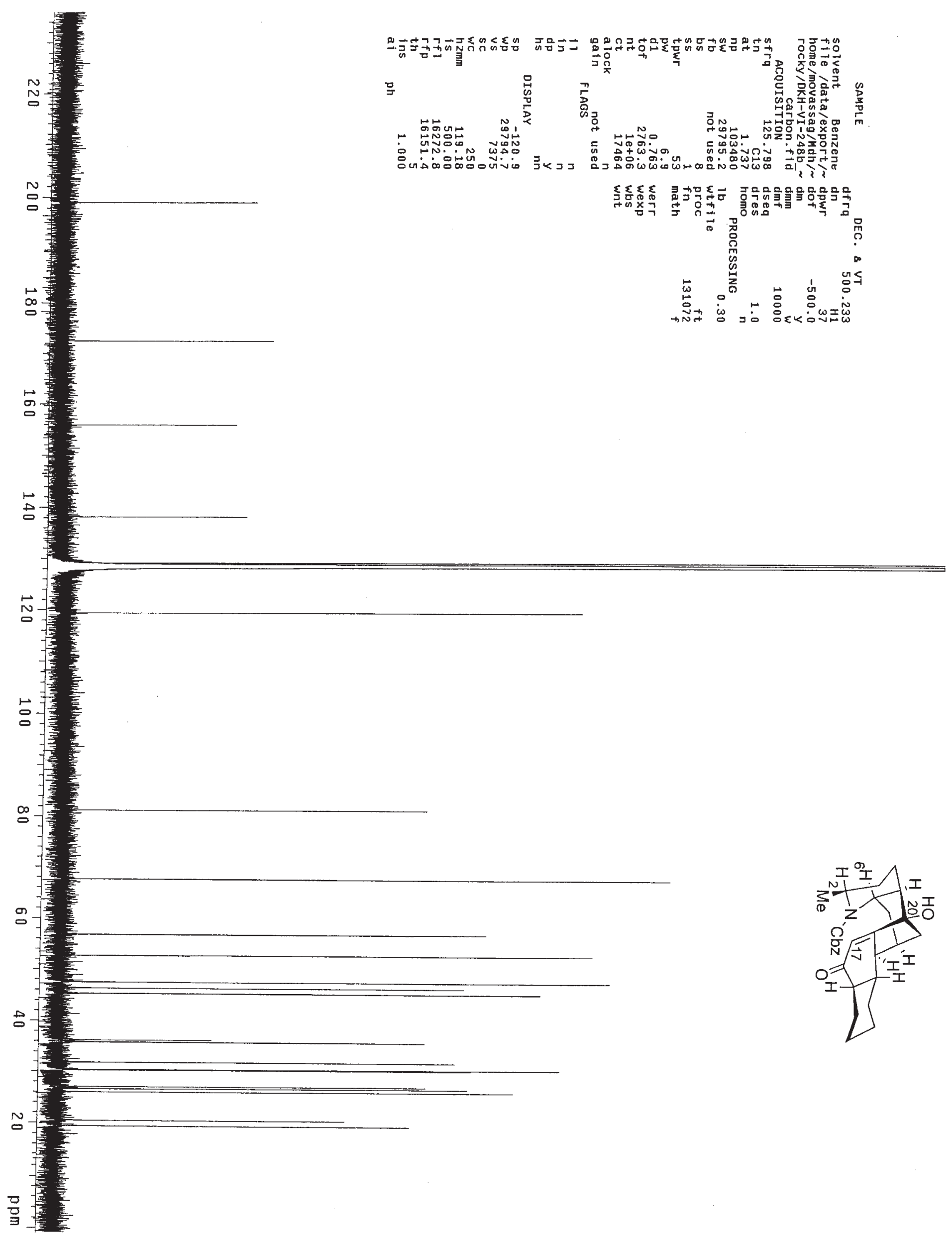




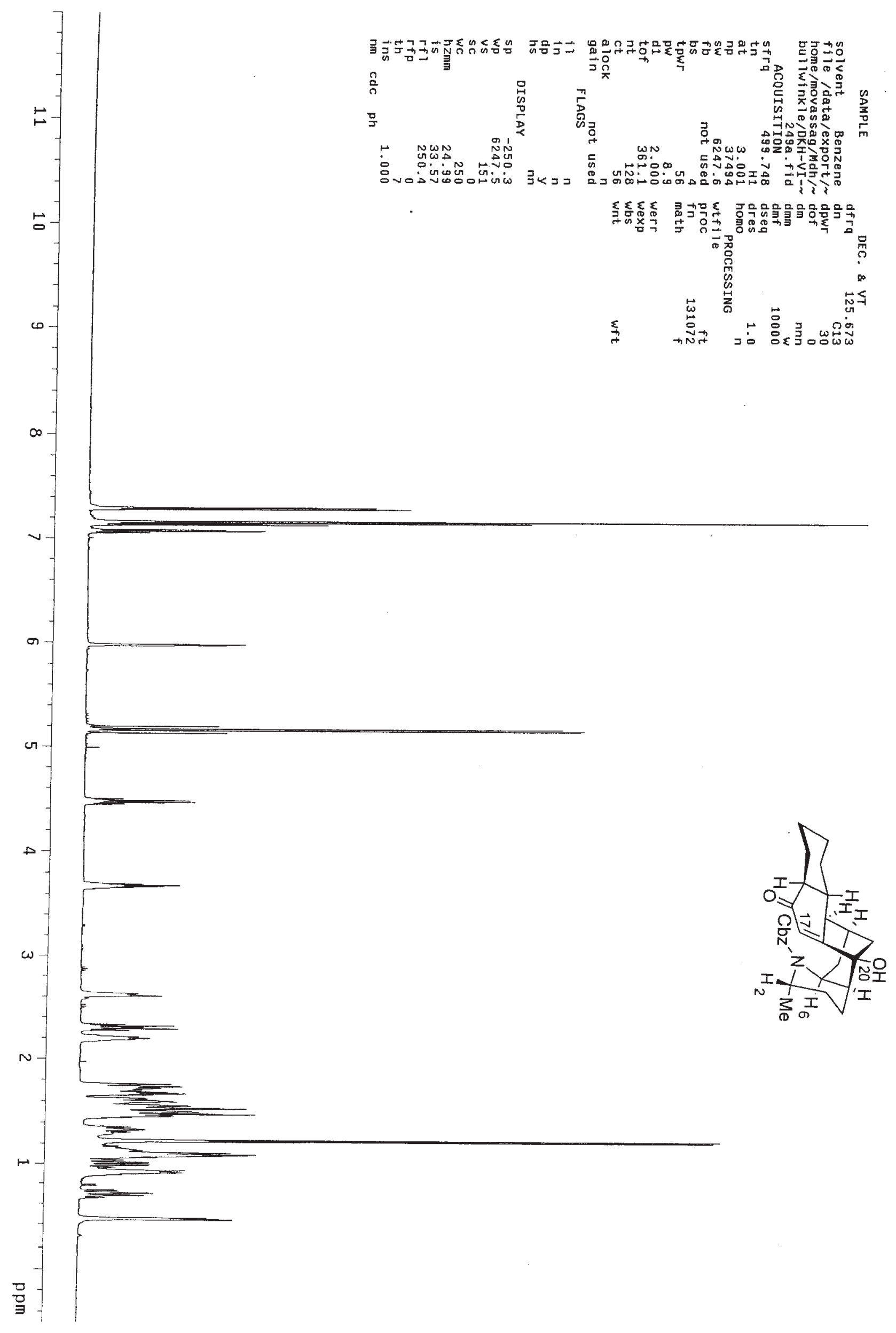




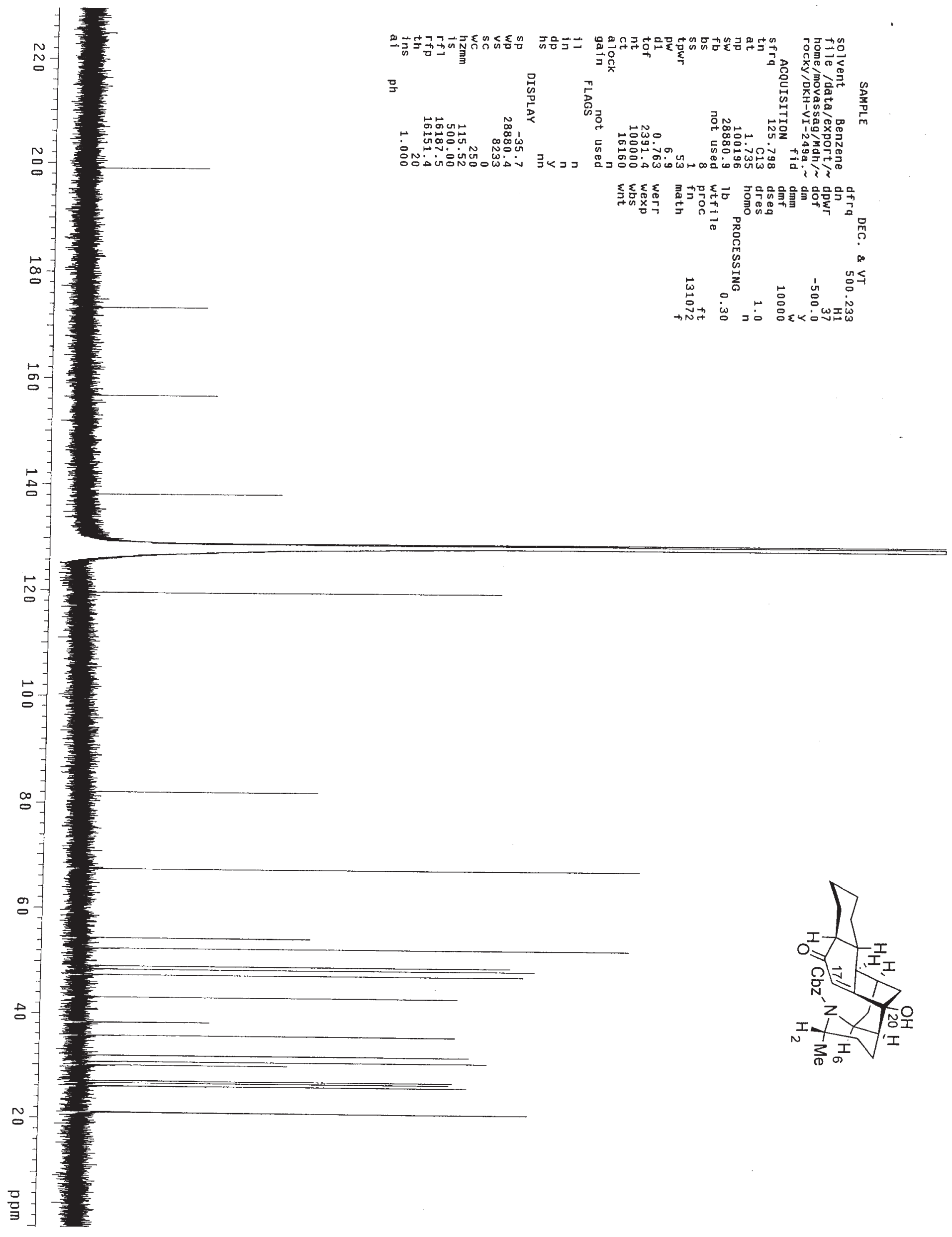




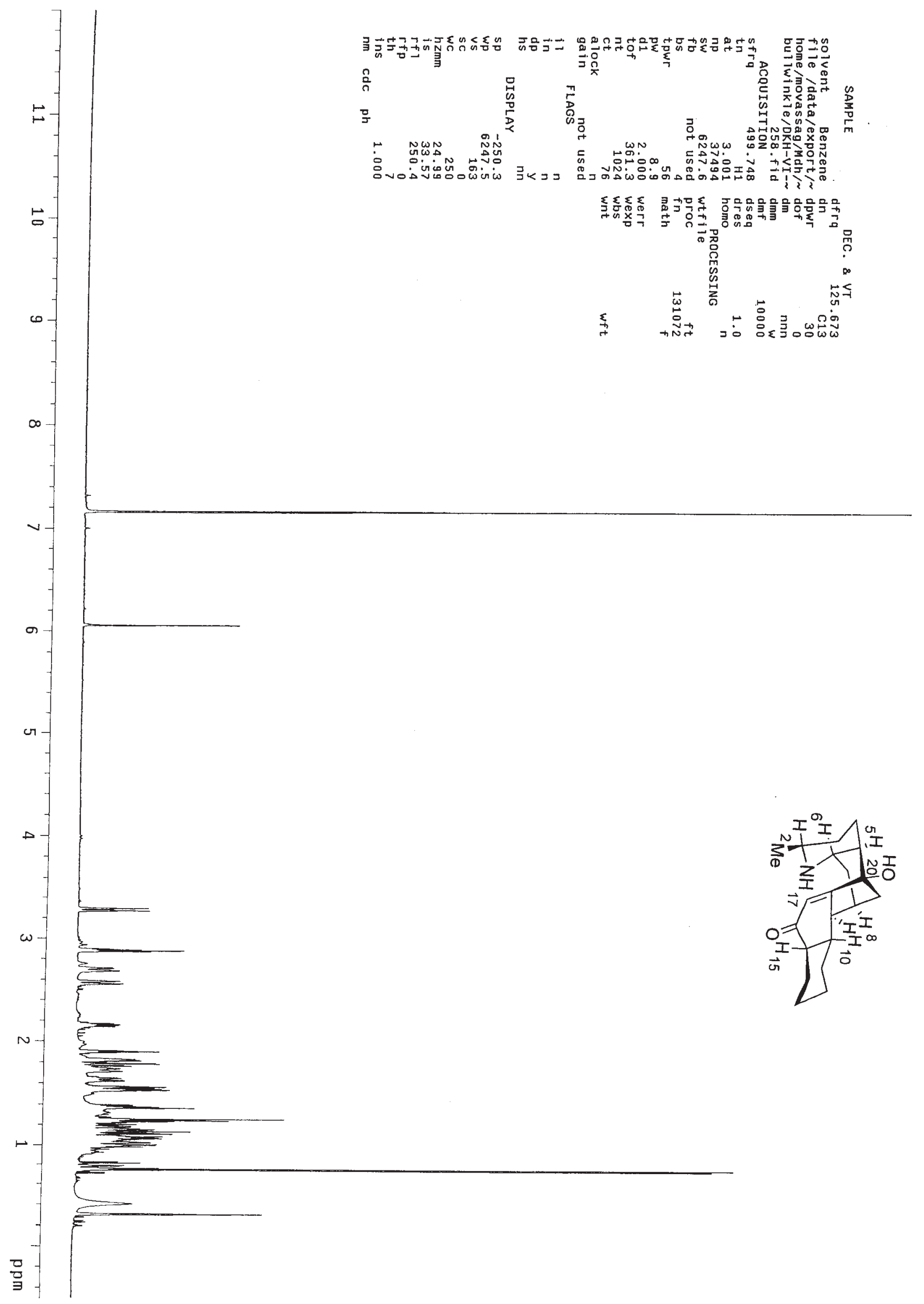




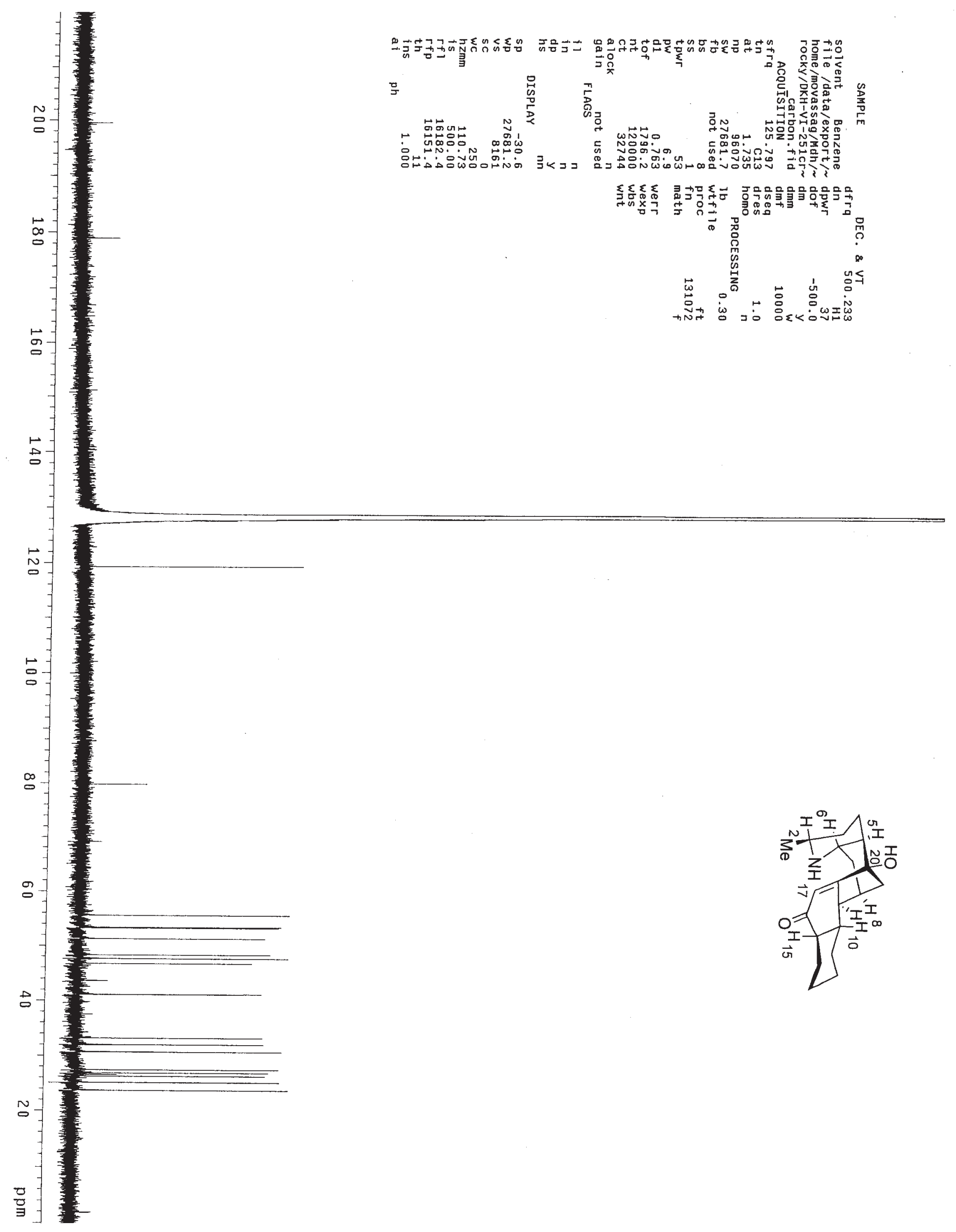




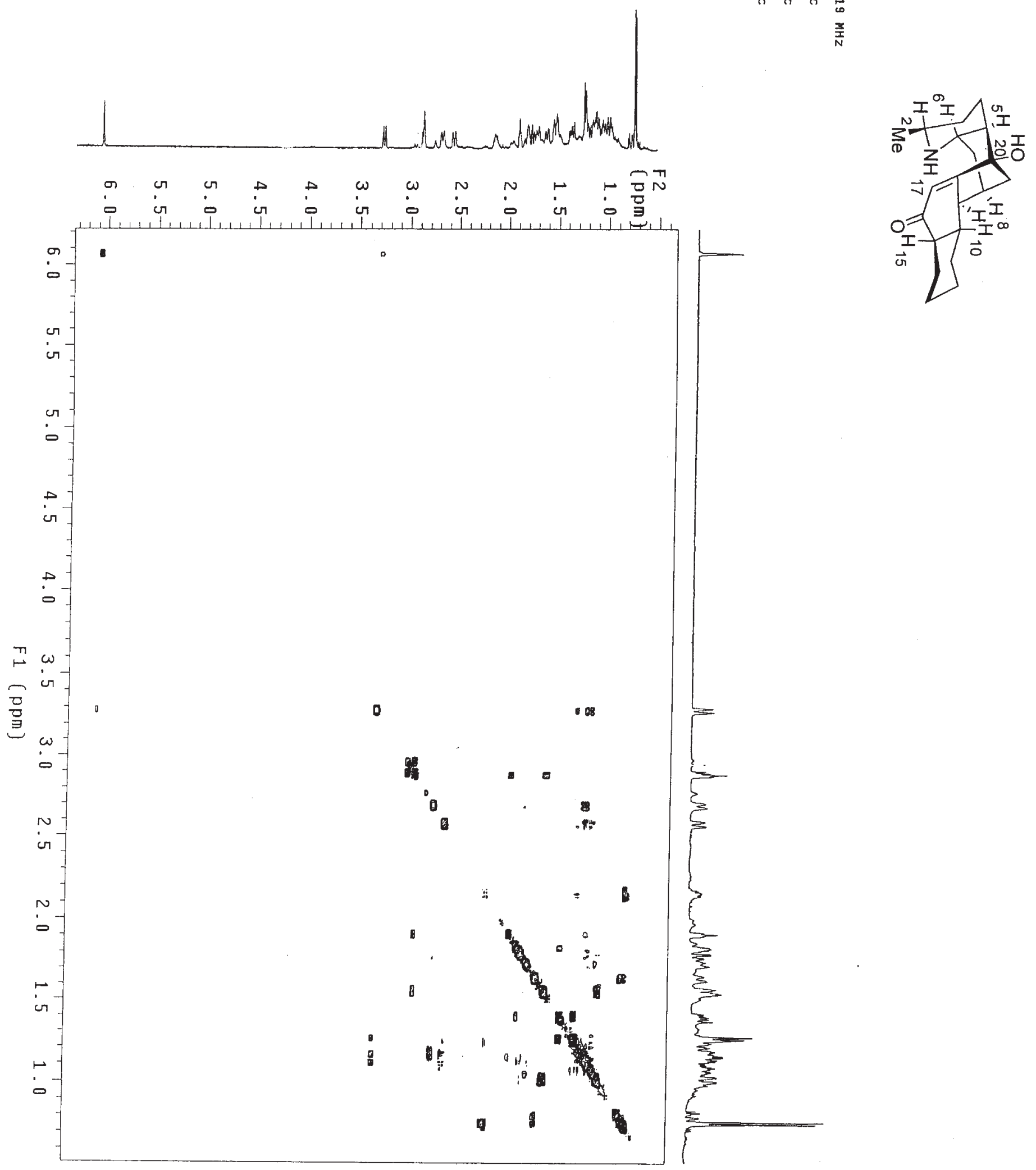




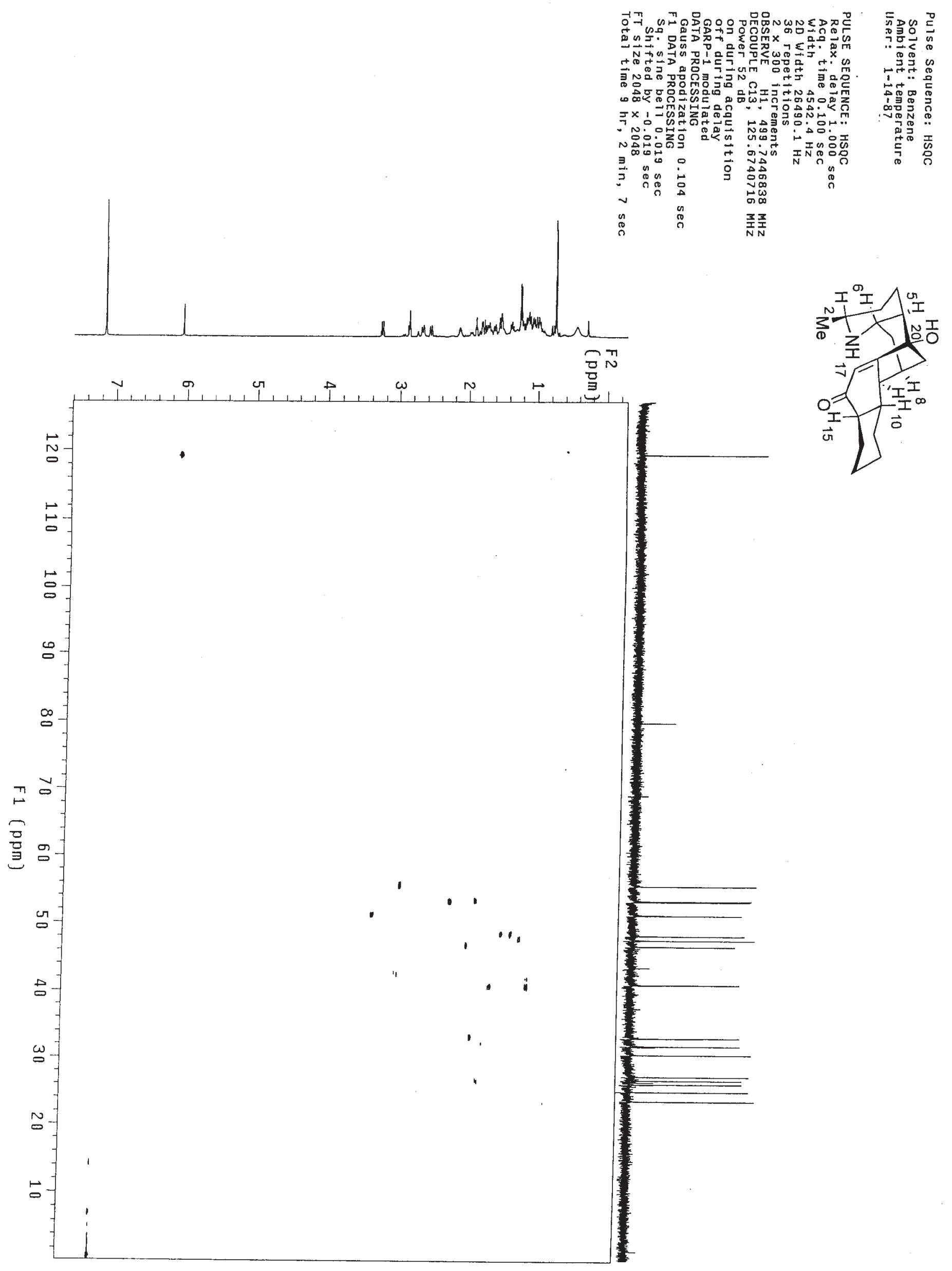

S87 


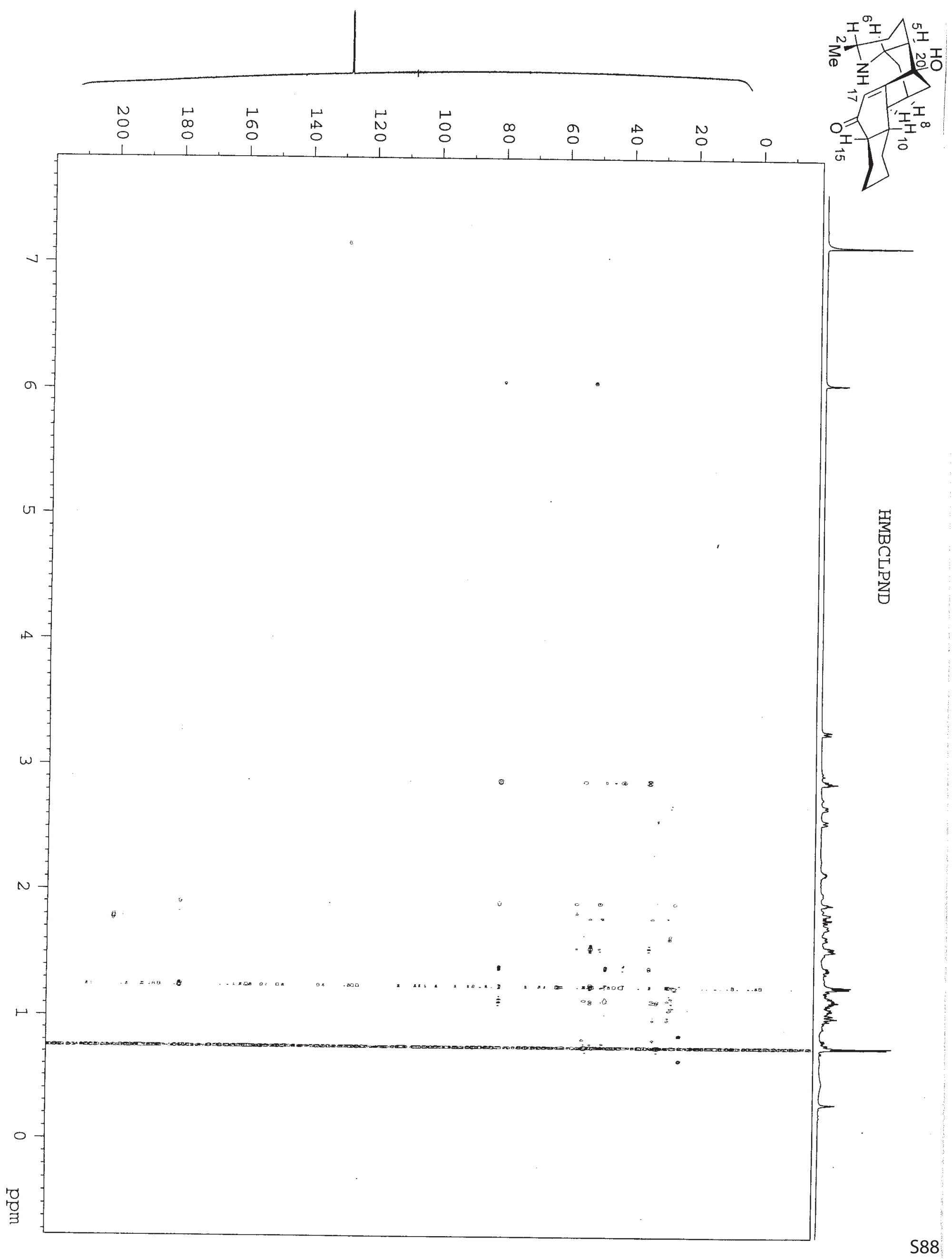




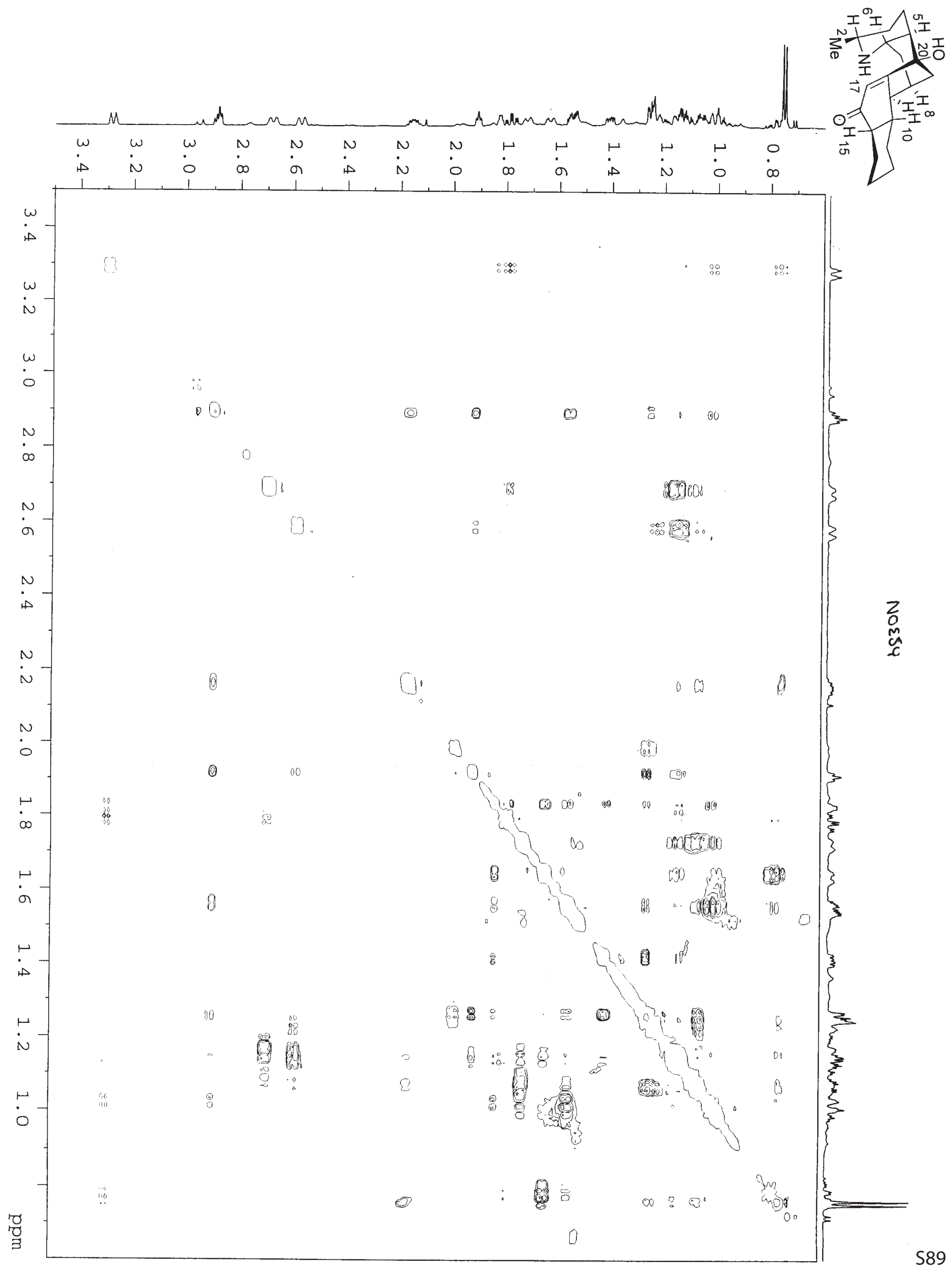




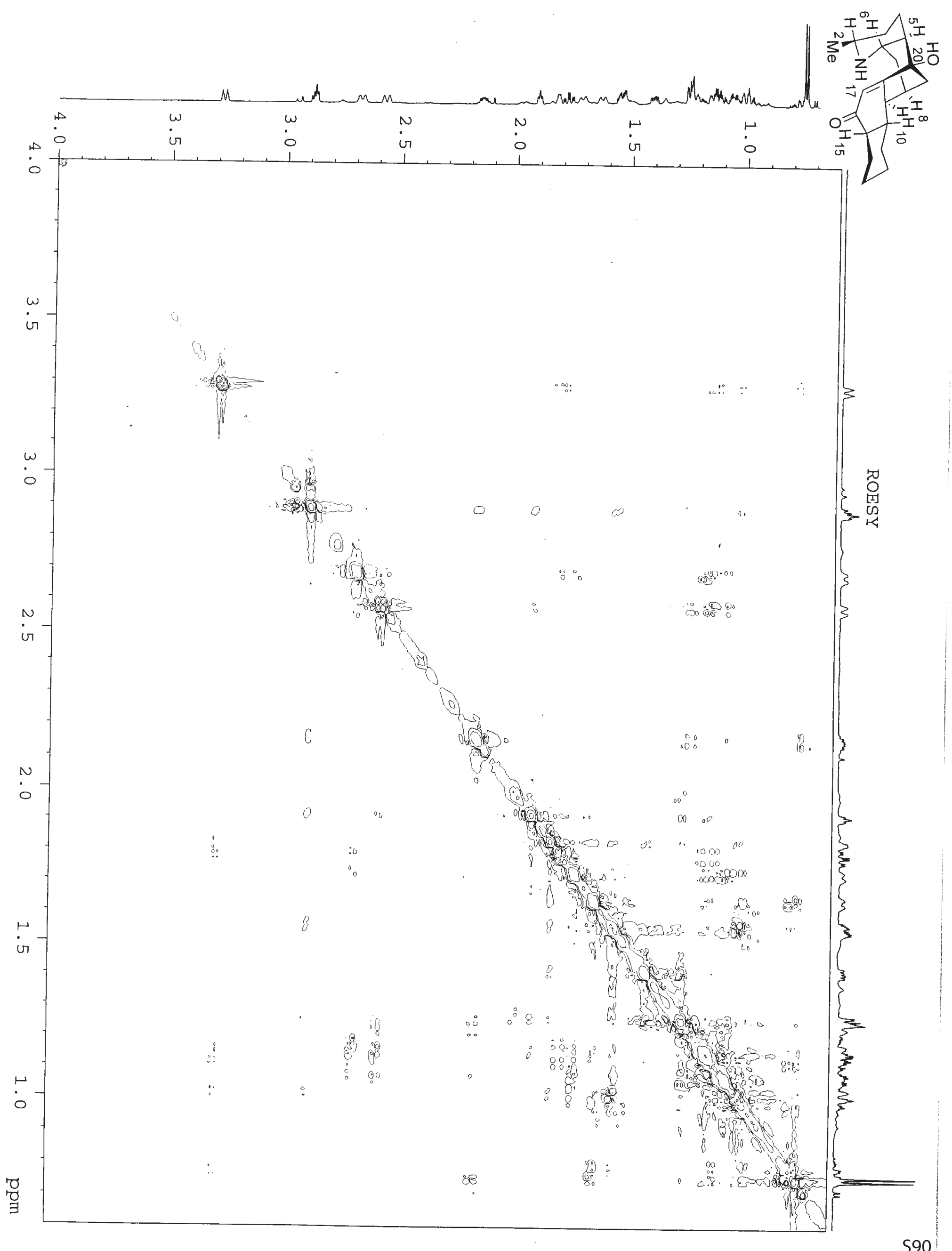



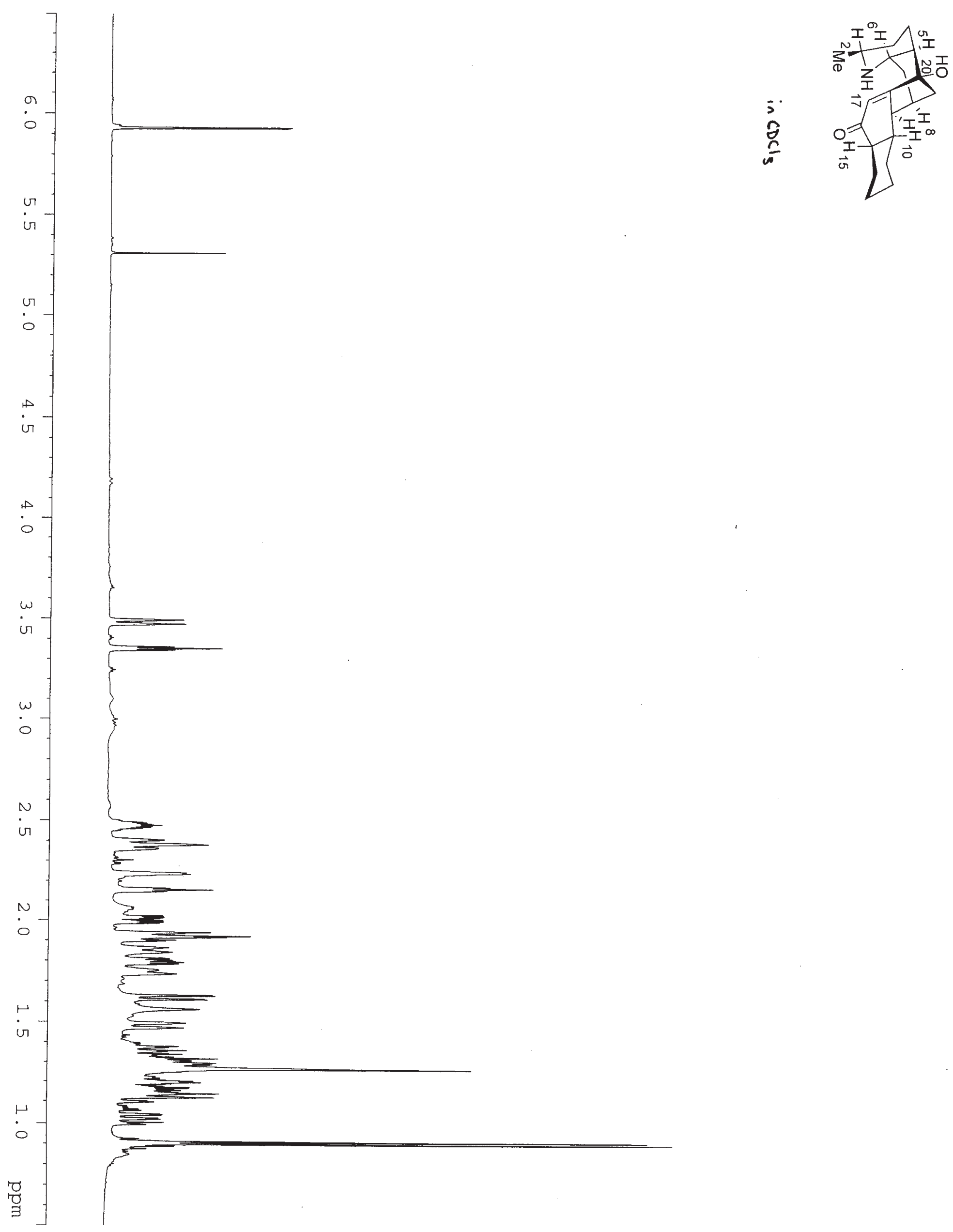

S91 


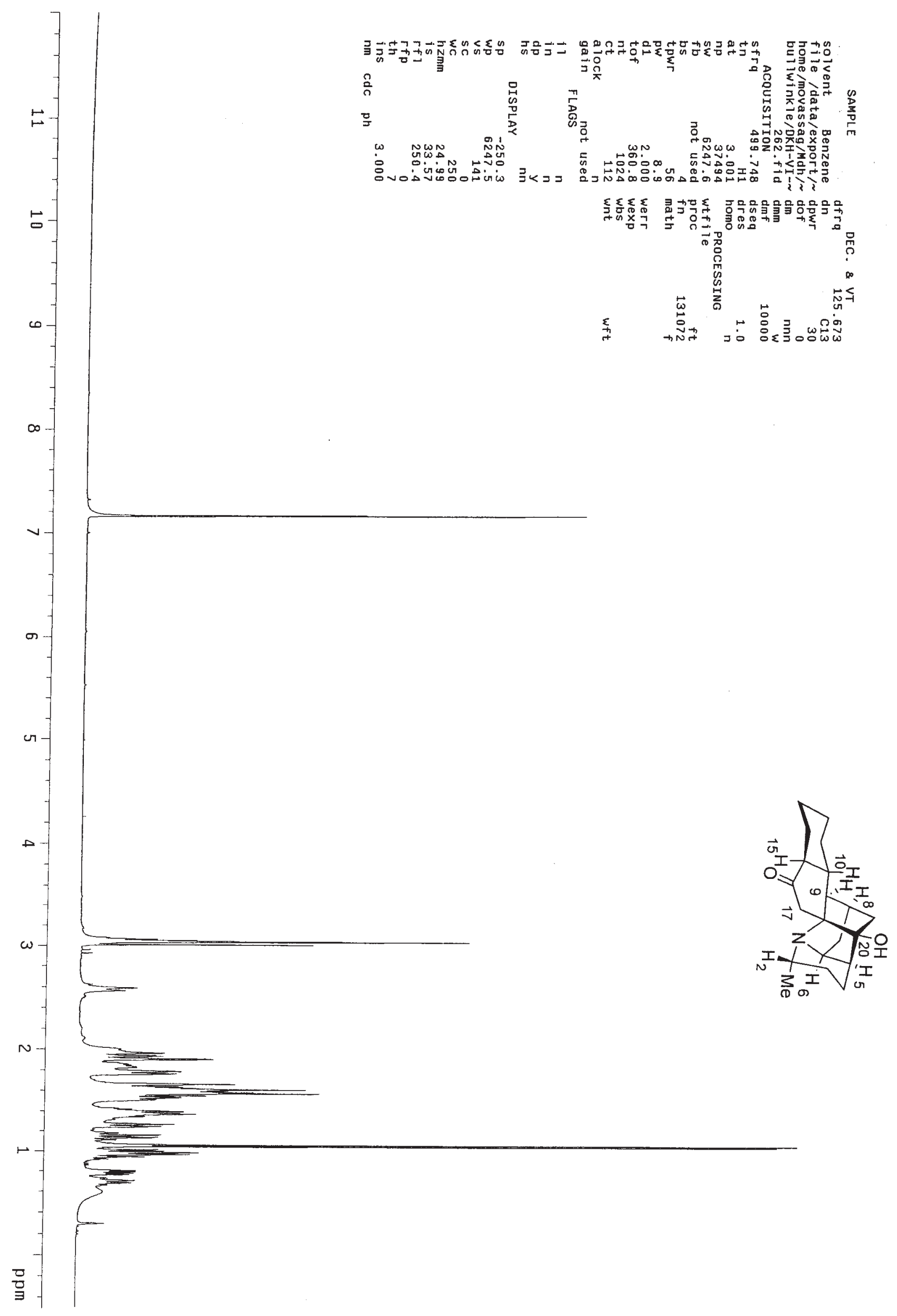




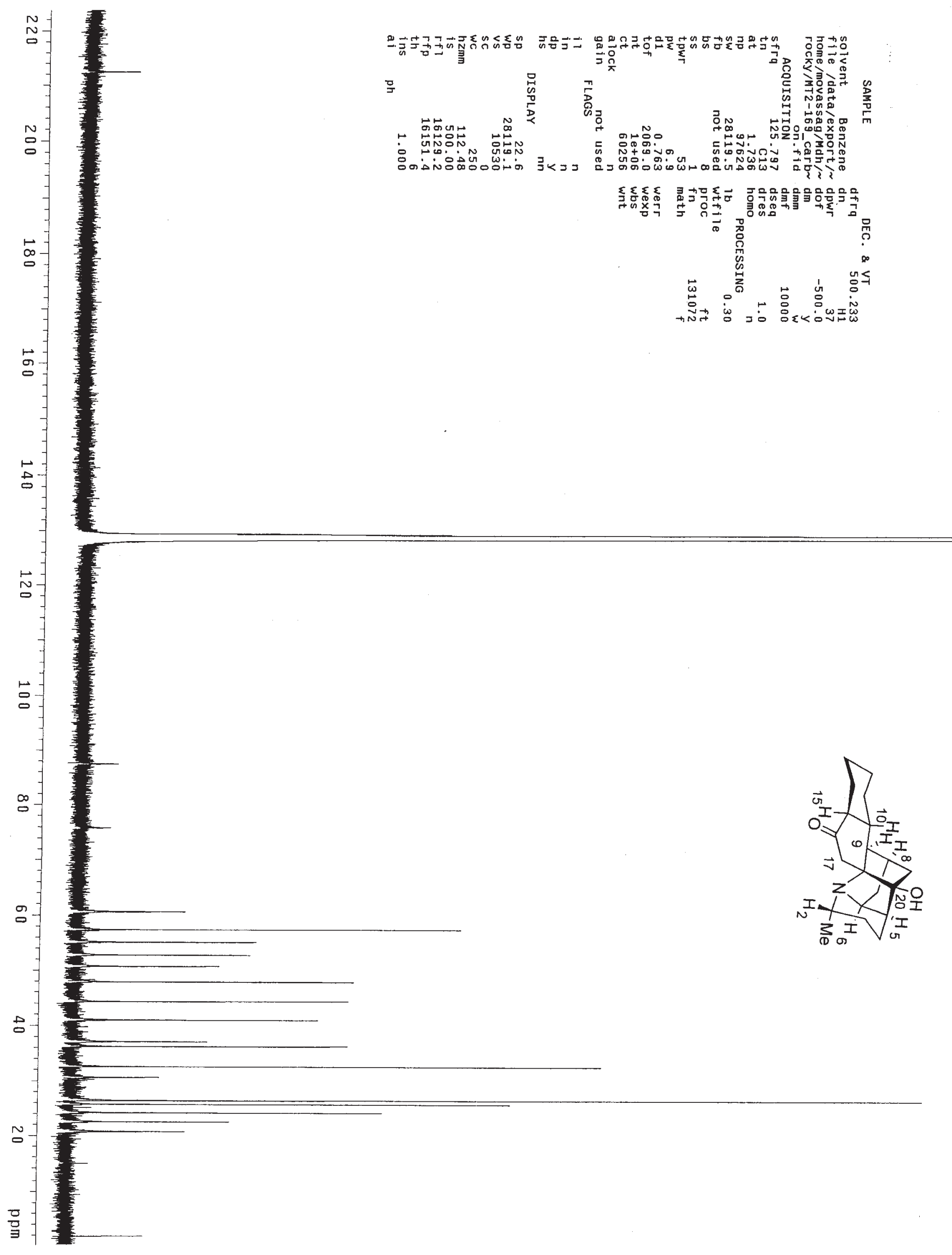




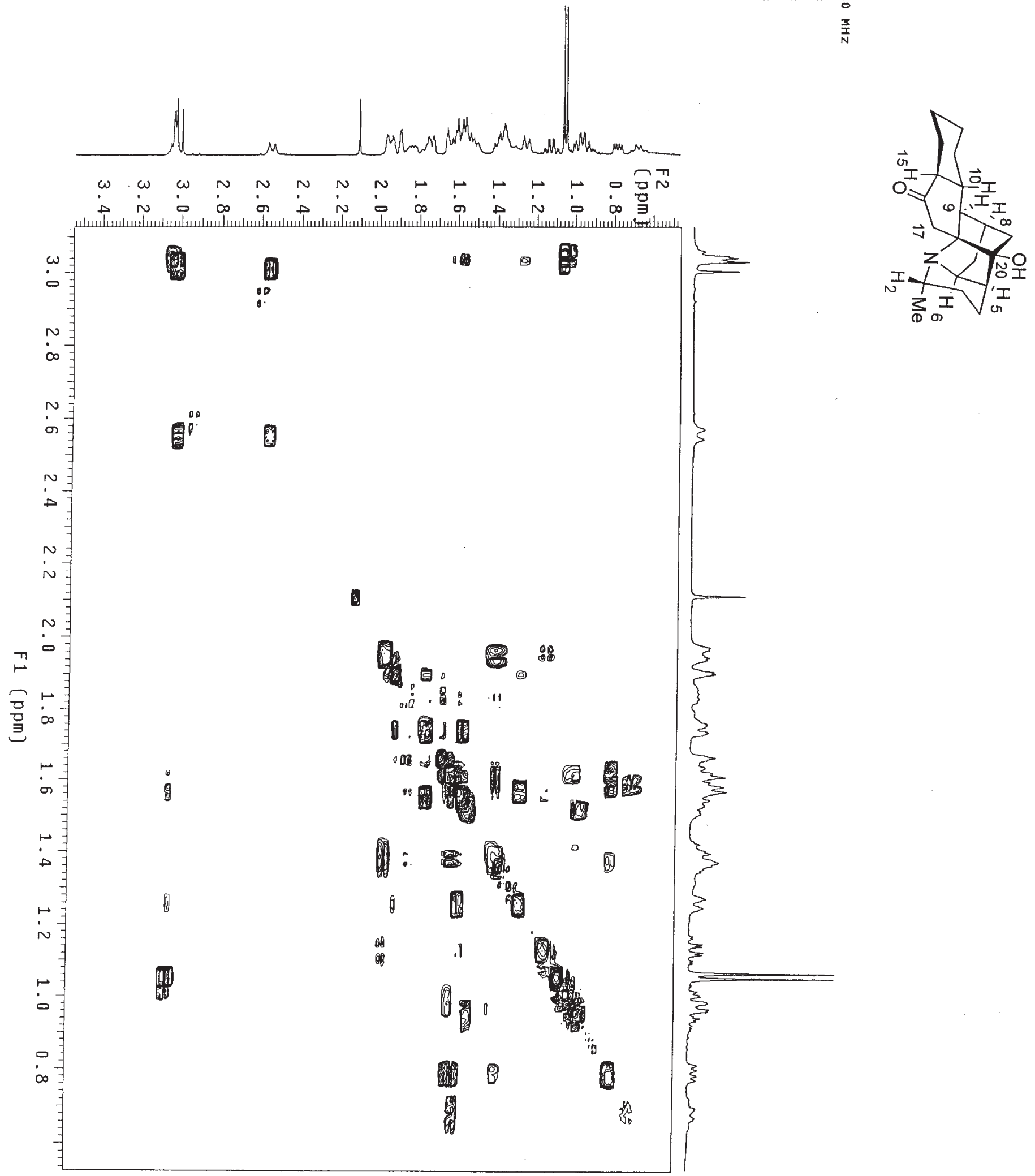




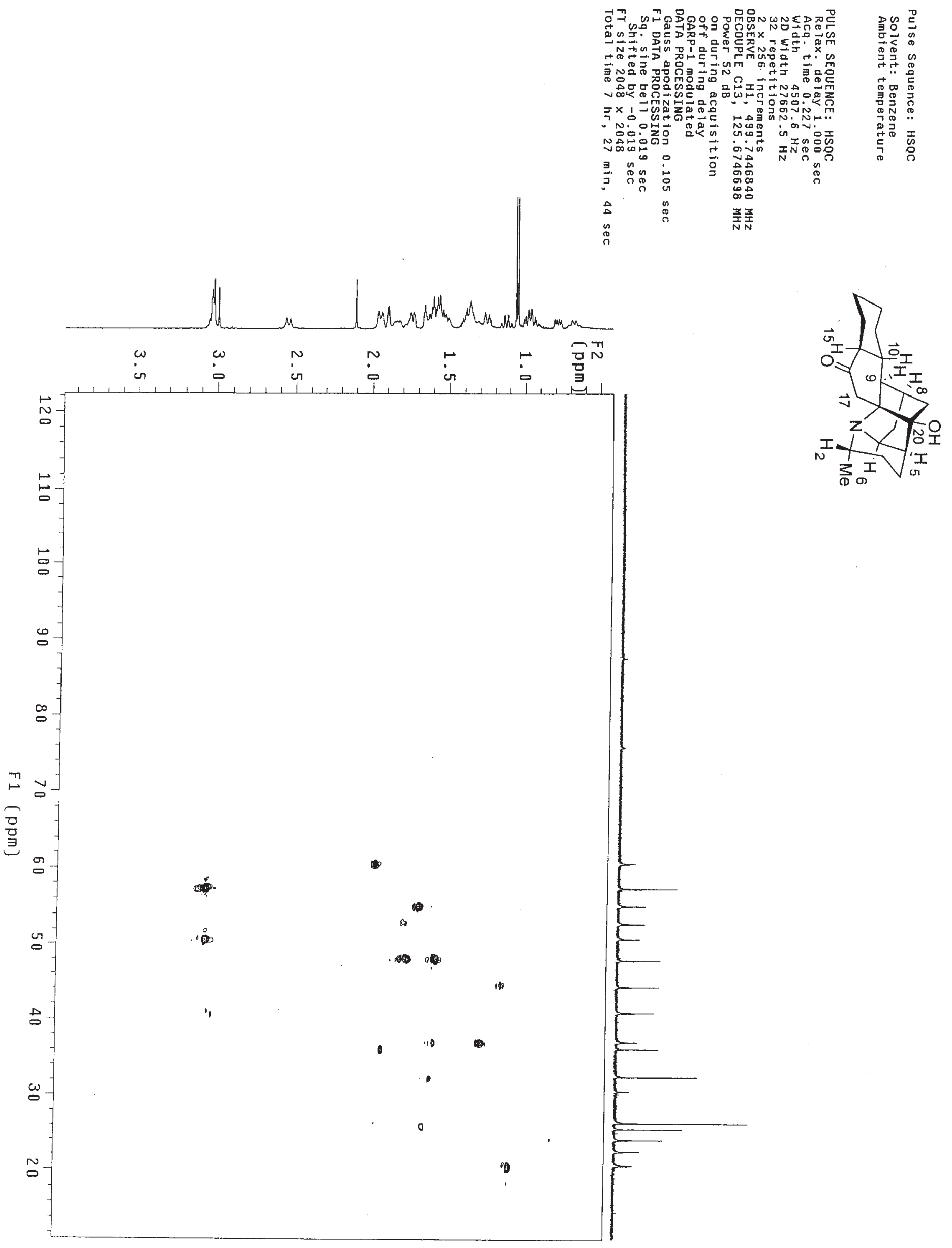




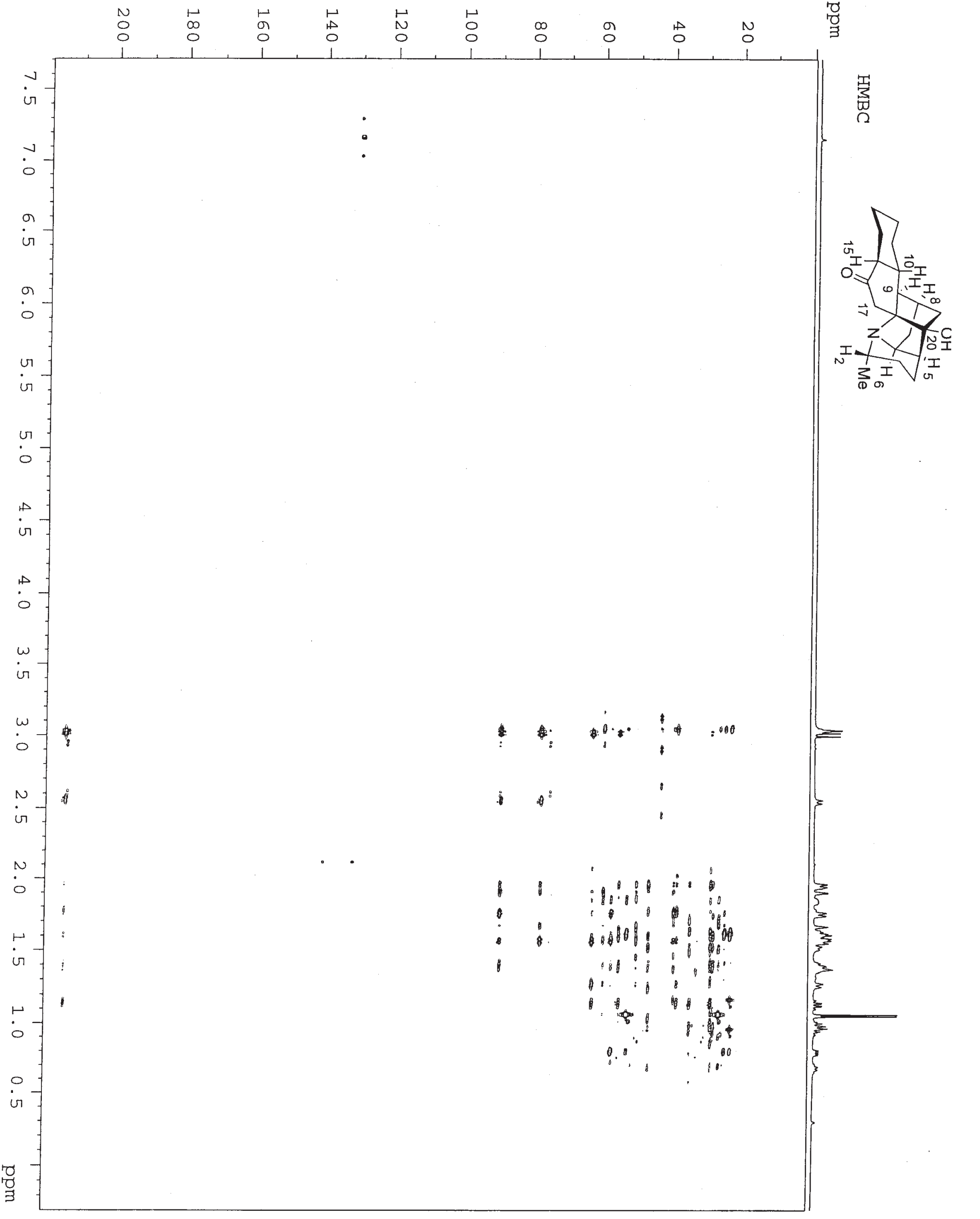




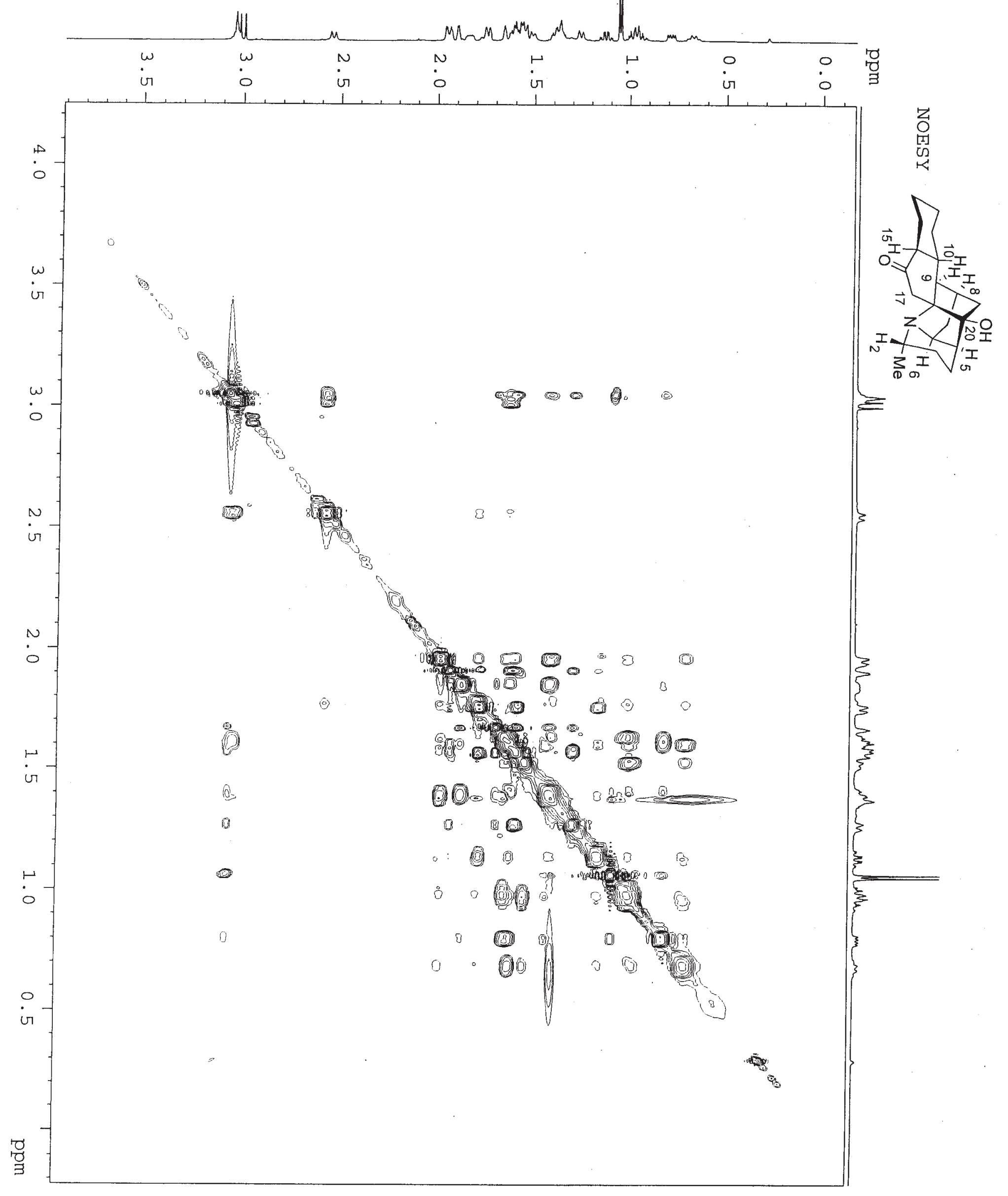




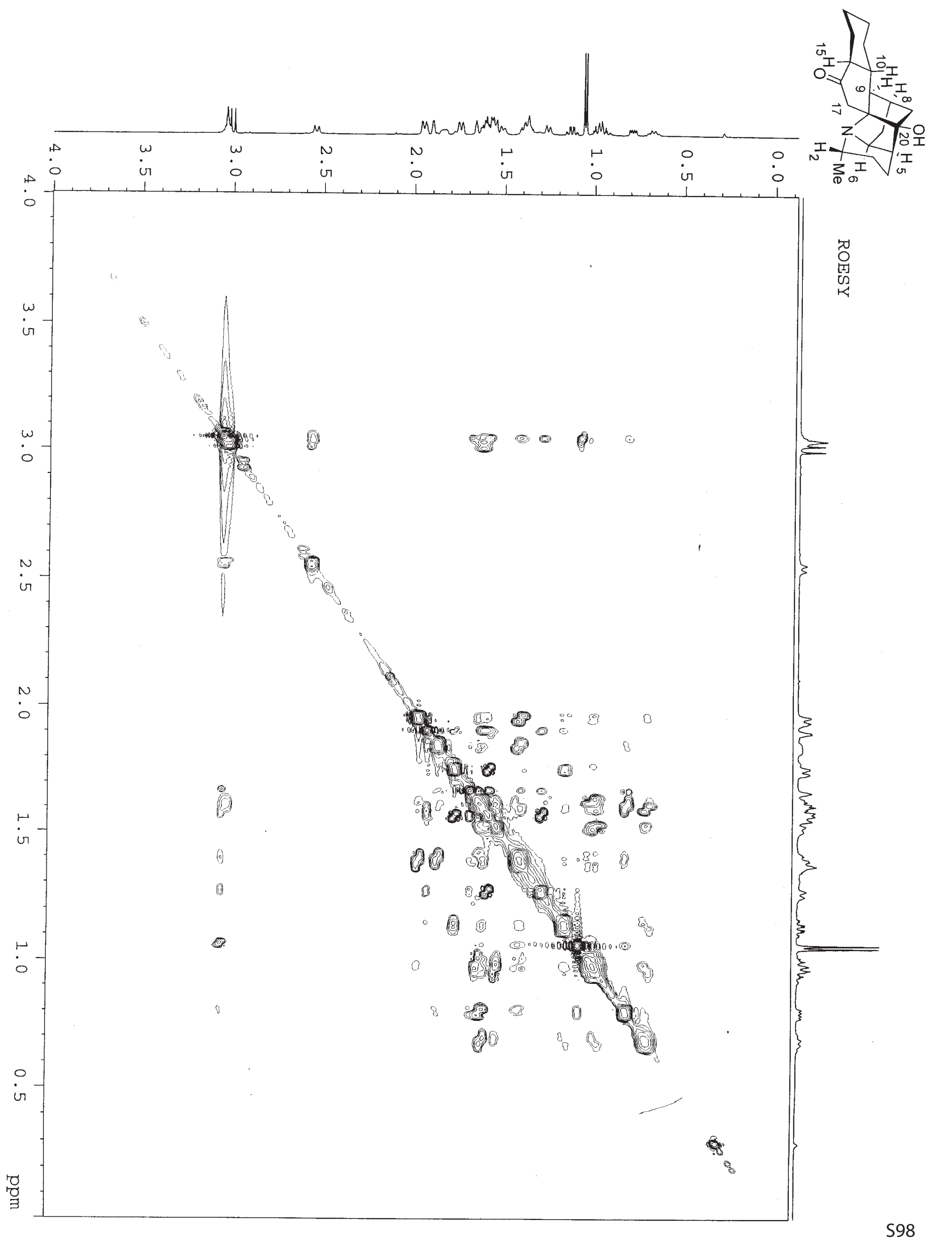

